\title{
Endolysosomal effectors and their relevance for antiviral activity against the Hepatitis E virus
}

\author{
Dissertation \\ To obtain the academic degree \\ Doctor in Science, Doctor philosophiae naturalis (Dr. phil. nat.) \\ Submitted to the \\ Department of Biochemistry, Chemistry and Pharmacy \\ Johann Wolfgang Goethe University \\ Frankfurt am Main - Germany
}

Presented by

Mirco Glitscher

Born in Darmstadt, Germany

Frankfurt am Main (2021)

(D30) 
vom Fachbereich Biochemie, Chemie, Pharmazie (14) der

Johann Wolfgang Goethe - Universität als Dissertation angenommen.

Dekan:

Prof. Dr. Clemens Glaubitz

Gutachter:

Prof. Dr. Robert Tampé

Prof. Dr. Eberhard Hildt

Datum der Disputation: $\quad$ 09.07.2021 
To all of those I do not cease to love and admire.

"It is important to go into work you would like to do. Then it does not seem like work. You sometimes feel it is almost too good to be true that someone will pay you for enjoying yourself."

-Gertrude B. Elion 


\section{Contents}

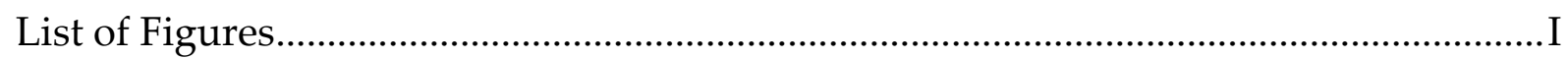

List of Abbreviations...................................................................................................

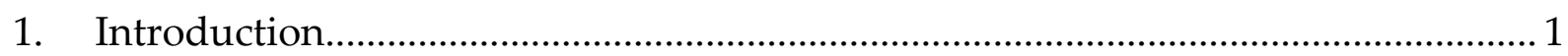

1.1. The Hepatitis E virus................................................................................. 1

1.1.1. Classification and phylogeny …………………………………………..... 1

1.1.2. Genomic organisation and viral proteins ................................................. 2

1.1.1. Transmission and epidemiology …………………...................................... 5

1.1.2. Pathogenesis and treatment......................................................................

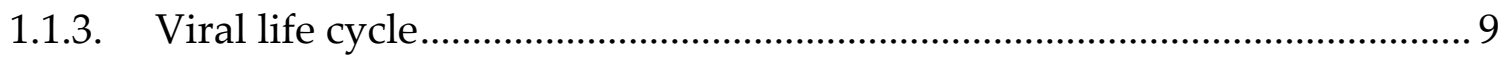

1.2. Non-systemic innate immunity .................................................................... 11

1.2.1. Pathogen-recognition receptors in viral infections .................................... 11

1.2.2. Cellular responses to interferons ............................................................ 13

1.2.3. Sensing of HEV and viral innate immune-evasion ..................................... 15

1.2.4. Guanylate-binding protein 1 .................................................................... 16

1.3. The endolysosomal system ………………................................................... 18

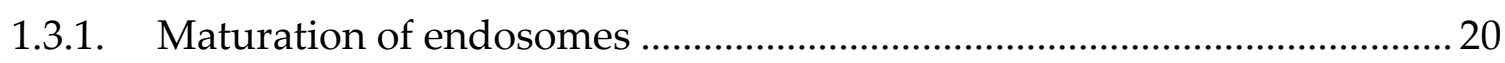

1.3.2. Cargo-sorting in MVBs with respect to HEV …………………………...... 22

1.3.3. Cholesterol-dependent regulations in the endolysosomal system .......... 25

1.4. Cellular cholesterol homeostasis and pharmacological modulation ...............27

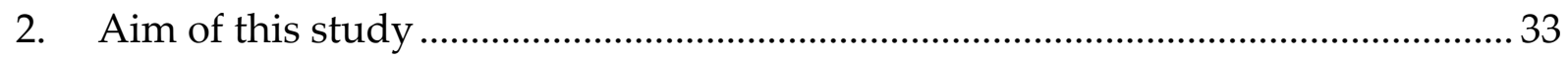

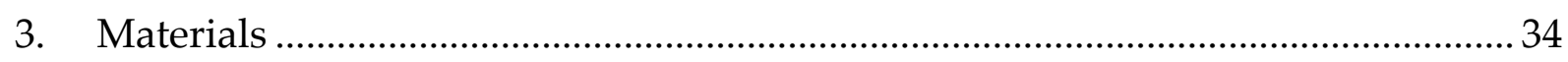

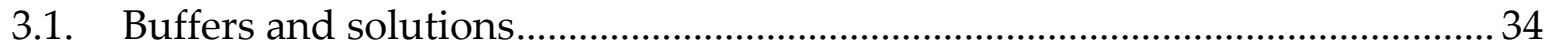

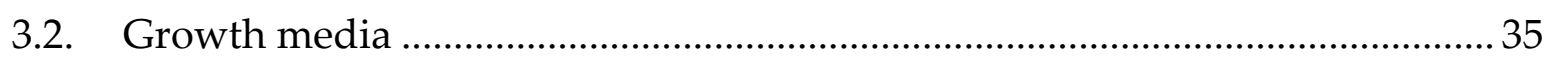

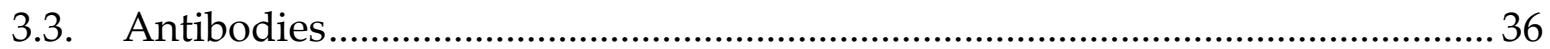

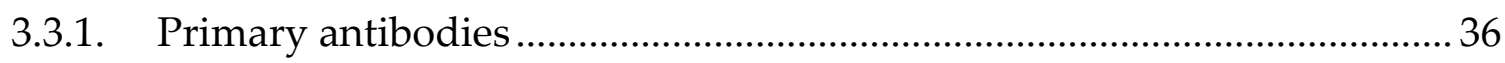

3.3.2. Secondary antibodies ................................................................................. 37

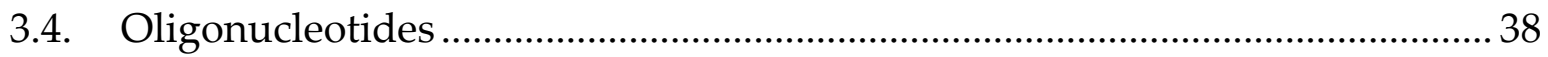

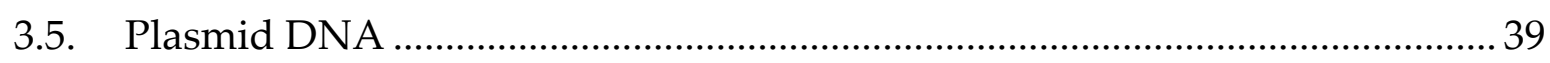

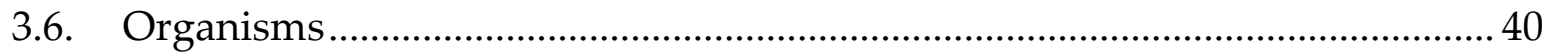

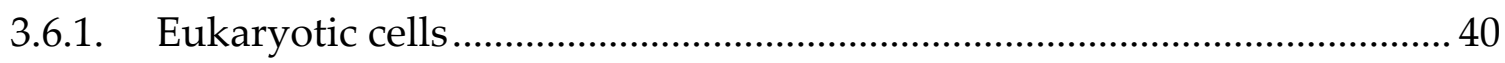

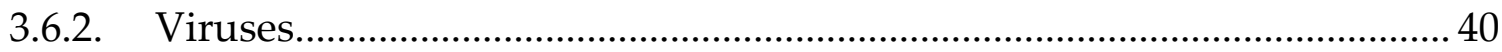




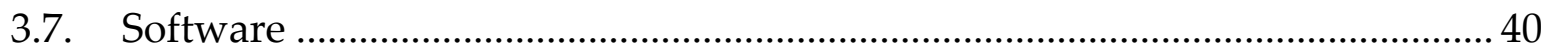

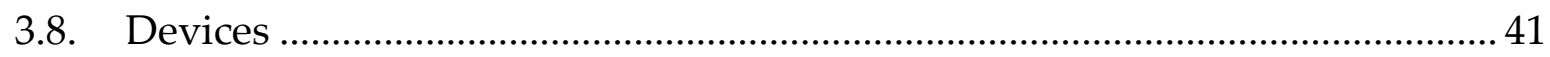

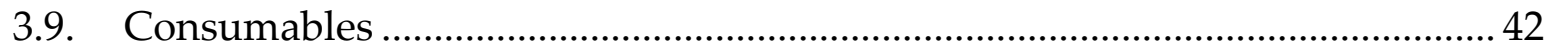

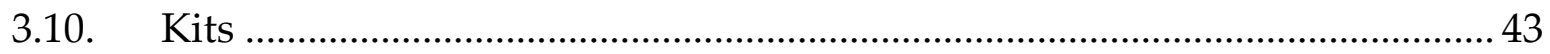

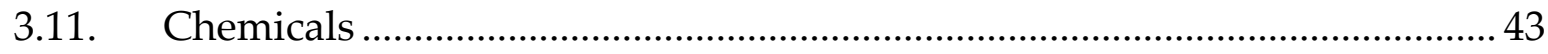

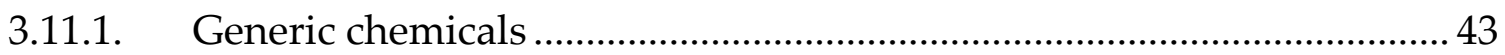

3.11.2. Chemicals used for treatments ....................................................... 45

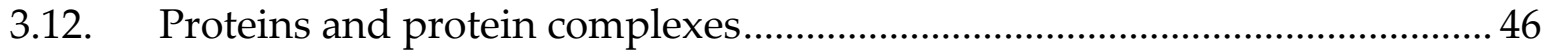

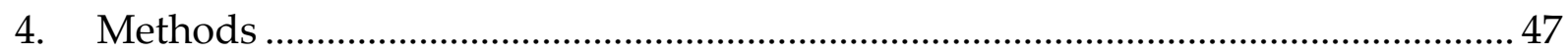

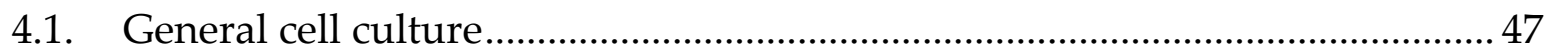

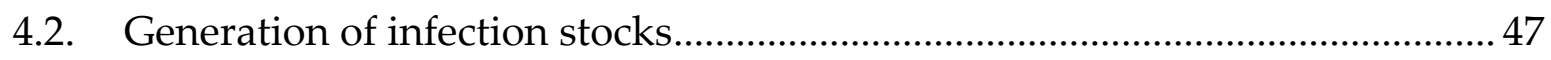

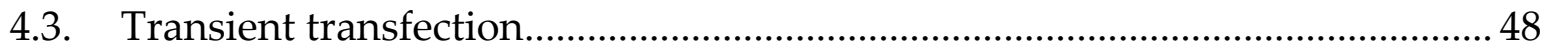

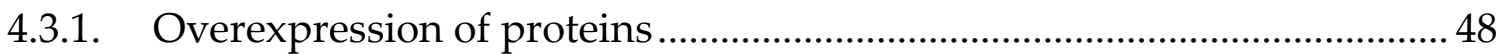

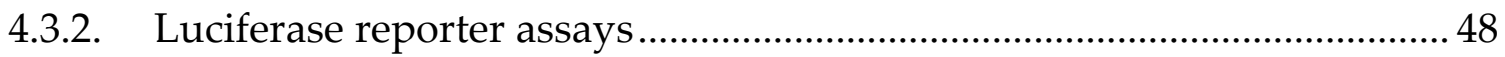

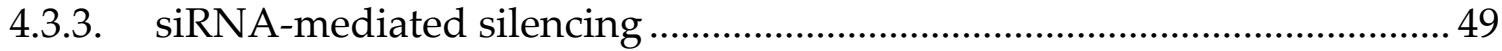

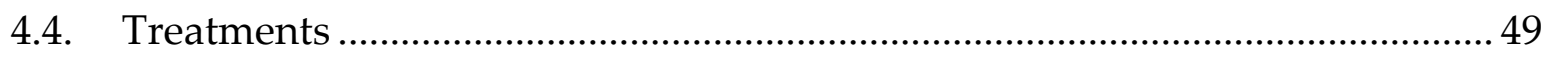

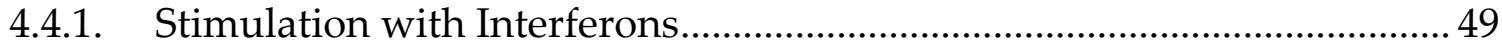

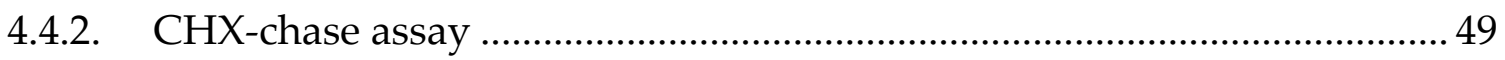

4.4.3. Elevation or reduction of intracellular cholesterol ................................... 50

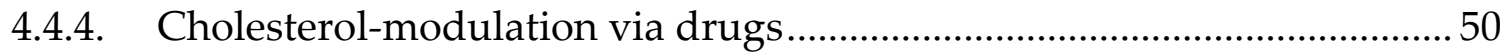

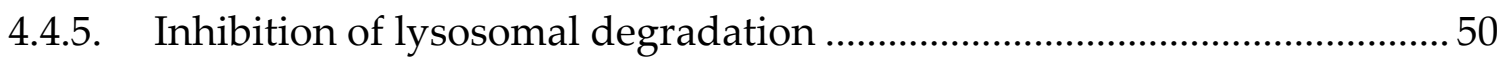

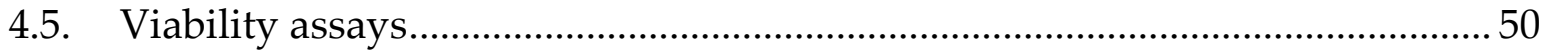

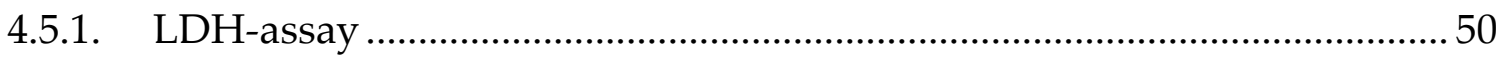

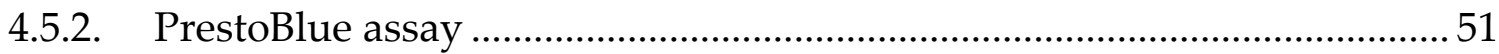

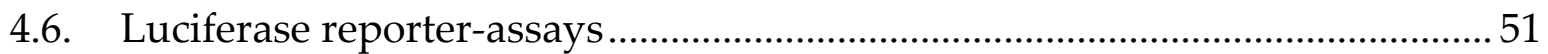

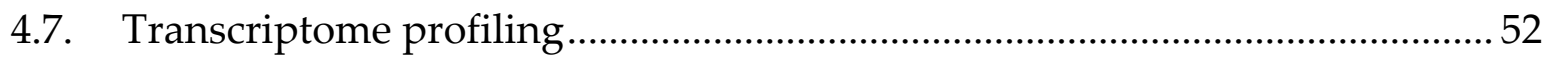

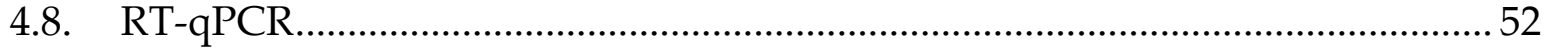

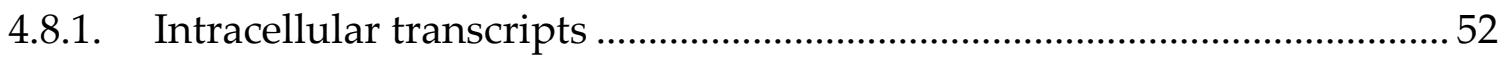

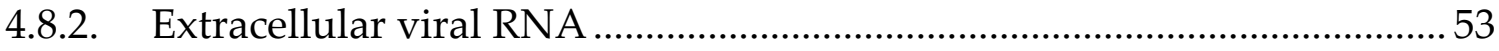

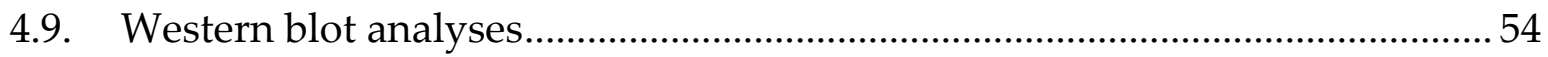

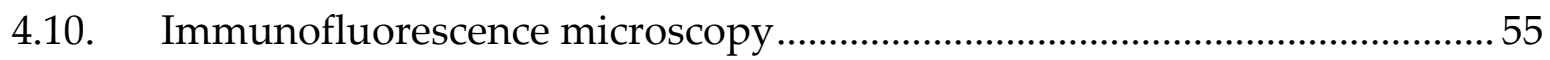

4.10.1. Immunofluorescent stain of ethanol-fixed samples ..............................55 
4.10.2. Immunofluorescent stain of formaldehyde-fixed samples....................56

4.10.3. Immunofluorescent stain with Filipin III ............................................ 56

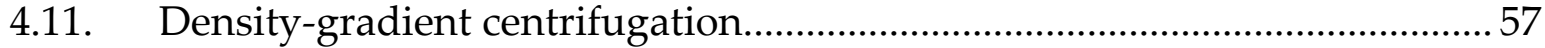

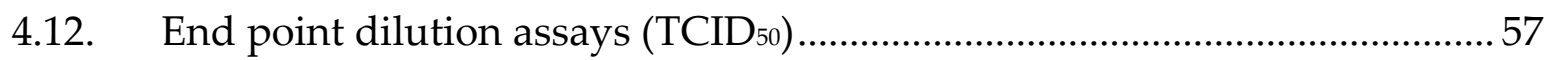

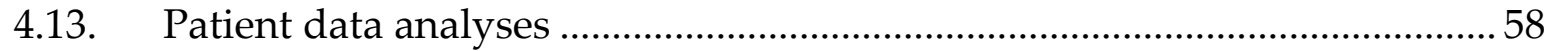

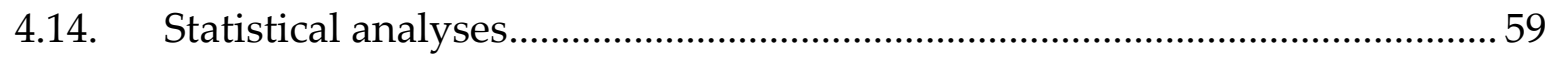

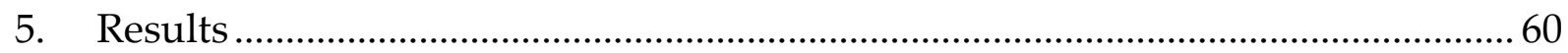

5.1. GBP1 induction and antiviral effect are most efficiently exerted by IFN $\gamma \ldots 60$

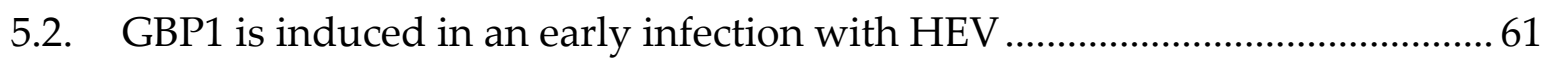

5.3. GBP1 is induced in a stable, persistent infection with HEV ............................ 63

5.4. GBP1 half-life is prolonged in HEV-infected cells ........................................... 65

5.5. Overexpression of GBP1 restricts HEV via lysosomal degradation ................66

5.6. GBP1 homodimerisation is required for lysosomal degradation of HEV ...... 68

5.7. Silencing of GBP1 rescues HEV from IFN $\gamma$-mediated lysosomal degradation 70

5.8. IFN $\gamma$ induces lysosomal cholesterol accumulation

5.9. HEV-infection alters expression of cholesterol-regulating genes and reduces

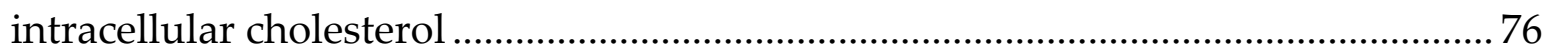

5.10. Serum-lipids are reduced in chronically HEV-infected patients .................78

5.11. A549 cells display a suitable model for studying modulations in cholesterolhomeostasis

5.12. Elevation of intracellular cholesterol restricts HEV

5.13. Elevated intracellular cholesterol induces lysosomal degradation of HEV83

5.14. Cholesterol-modulating drugs act efficiently in A549 cells....

5.15. Drug-induced elevation of intracellular cholesterol impairs viral release. 90

5.16. Efficiently acting cholesterol-modulators induce lysosomal degradation of HEV 93

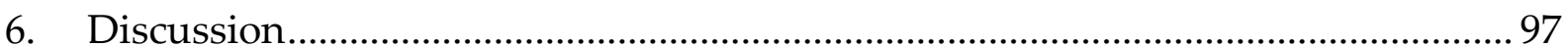

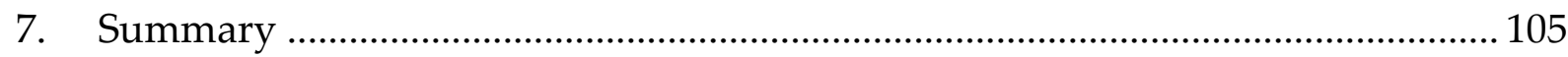

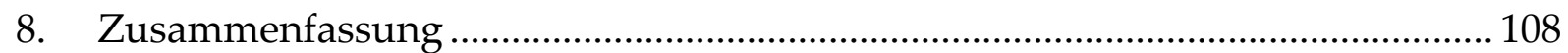

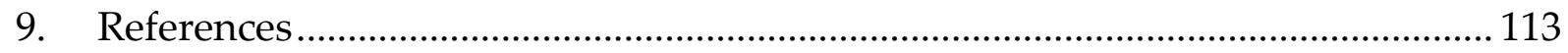

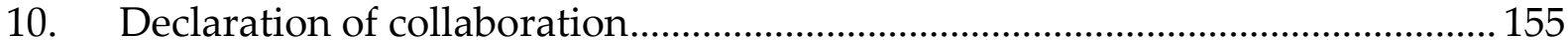




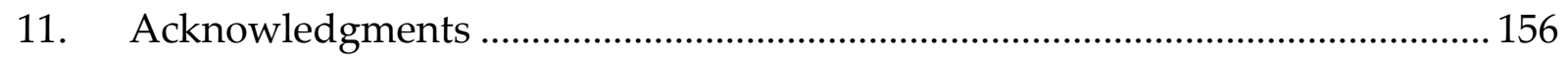

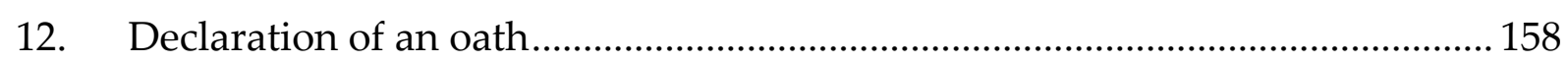

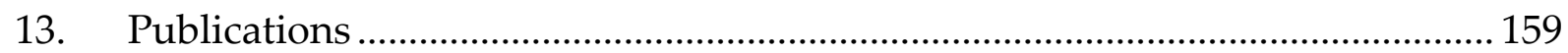

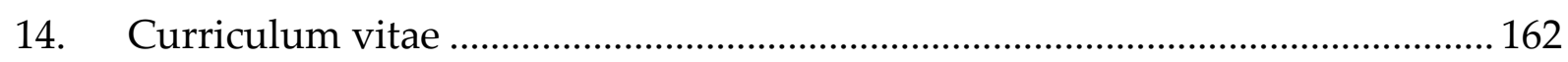




\section{List of Figures}

Figure 1. Phylogenetic tree of the family Hepeviridae. .............................................. 2

Figure 2. Genomic and subgenomic organisation of HEV. ...................................... 3

Figure 3. HEV proteins and their functional domains and post-translational modifications.

Figure 4. Sources of zoonotic and human-human transmitted HEV-infection. .......... 6

Figure 5. Geographical spread of human-pathogenic HEV. ..................................... 7

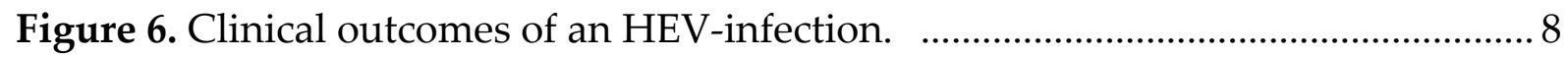

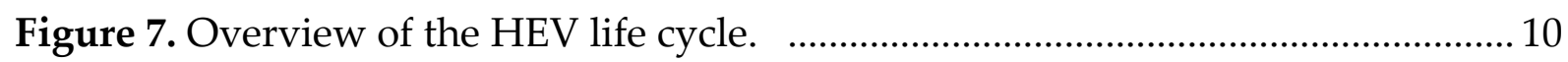

Figure 8. Pathogen-recognition receptor-mediated sensing of RNA viruses and

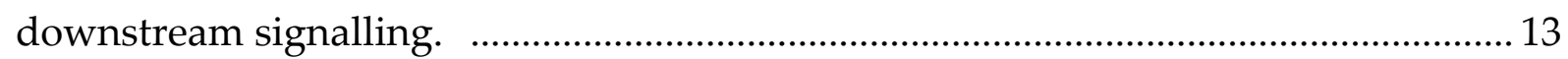

Figure 9. Type I-III IFN signalling pathways for transcriptional regulation. ............ 14

Figure 10. Innate sensing of HEV and virus-related interferences. ............................ 16

Figure 11. Oligomeric organisation of GBP1 and implications for membrane-targeted

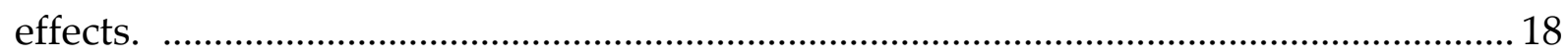

Figure 12. Overview of the endolysosomal system. …................................................ 19

Figure 13. Rab5 and its effectors on the membrane of early endosomes. ................... 21

Figure 14. Endosome maturation in dependency of Rab-proteins and

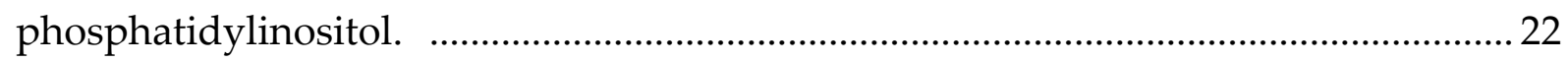

Figure 15. Ubiquitin-dependent cargo-sorting and ILV budding on MVBs via the

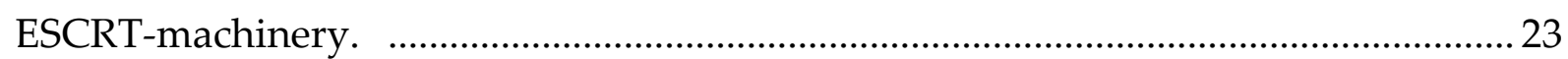

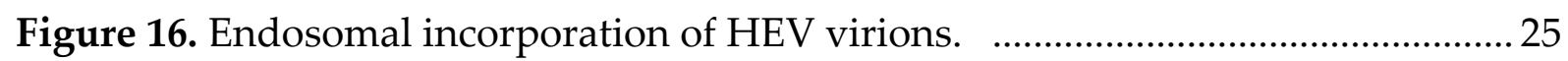

Figure 17. Regulatory processes of cholesterol in the endolysosomal system. ..........27

Figure 18. LDL-uptake and its resulting transcriptional changes. .............................. 28

Figure 19. Cholesterol-removal processes in a human hepatocyte. ............................ 31

Figure 20. IFN $\gamma$ induces GBP1 promoter and gene-expression most efficiently leading to strongest antiviral effect.

Figure 21. GBP1 mRNA and protein amount is modulated upon early HEV-infection

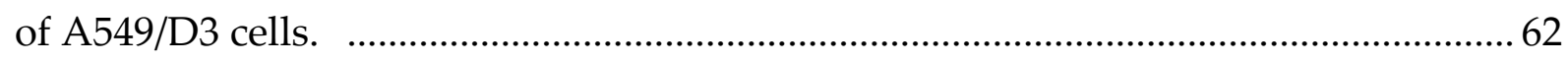

Figure 22. Induction of GBP1 is due to successful HEV-infection. .............................63

Figure 23. GBP1 promoter activity, mRNA and protein amount is modulated in persistently HEV-infected A549 cells.

Figure 24. Stabilising effect on GBP1 half-life exerted by an HEV-infection in A549 cells.

Figure 25. Overexpression of wild-type GBP1 reduces viral release from persistently HEV-infected A549 cells.

Figure 26. GBP1 overexpression induces lysosomal localisation of HEV and HEV pORF2 reduction rescuable via Leupeptin treatment. 
Figure 27. GBP1-S73A mutant fails to induce lysosomal HEV degradation while GBP1$\mathrm{R} 48 \mathrm{~A}$ retains antiviral activity.

Figure 28. IFN $\gamma$-mediated impairment of HEV life cycle is abrogated upon GBP1 silencing.

Figure 29. Silencing of GBP1 rescues HEV from IFN $\gamma$-mediated lysosomal localisation and HEV pORF2 reduction.

Figure 30. IFN $\gamma$-mediated lysosomal HEV encapsulation is abrogated upon GBP1 silencing.

Figure 31. IFN $\gamma$ induces changes in expression of genes encoding proteins involved in maintenance of cholesterol-homeostasis. 74

Figure 32. Type I and II Interferons elevate intracellular cholesterol and lead to its lysosomal accumulation. .75

Figure 33. HEV-infection triggers changes in cholesterol-related gene-expression and a reduction in intracellular cholesterol. .77

Figure 34. Early HEV-infection triggers reduction of serum-lipids in renal transplant patients. .78

Figure 35. Cholesterol-induced gene-expression in A549 cells behaves similar to Huh7 cells.

Figure 36. Intracellular cholesterol can be modulated with LDL, 25-HC or Simvastatin in A549 cells.

Figure 37. Increased intracellular cholesterol concentrations lead to reduction in intracellular HEV pORF2.

Figure 38. High intracellular cholesterol reduces viral release, whereas low intracellular cholesterol is in favour of it.

Figure 39. Modulation of intracellular cholesterol reduces intracellular HEV pORF2 and affects its lysosomal localisation.

Figure 40. Blocking of lysosomal degradation rescues intracellular HEV pORF2 from cholesterol-induced reduction. 85

Figure 41. Cholesterol modulating drugs are non-toxic to A549 cells. .87

Figure 42. Cholesterol modulating drugs display slight cytostatic effects in A549 cells.

Figure 43. PSC833 and Fenofibrate efficiently increase intracellular cholesterol in A549 cells.

Figure 44. PSC833 and Fenofibrate act similarly on A549 cells as on Huh-7 cells. ...90 Figure 45. Cholesterol-modulating drugs efficiently inhibit viral release from persistently HEV-infected A549 cells.

Figure 46. PSC833 and Fenofibrate prohibit efficient release of infectious viral particles from persistently HEV-infected A549 cells.

Figure 47. PSC833 and Fenofibrate reduce intracellular amount of HEV pORF2. .... 93 
Figure 48. PSC833 and Fenofibrate efficiently reduce intracellular HEV pORF2 and induce its lysosomal localisation. 94 Figure 49. Blocking of lysosomal degradation rescues HEV pORF2 from drug-induced reduction. .95

Figure 50. Schematic representation of antiviral activities identified in this study. 107 
List of Abbreviations

\begin{tabular}{|c|c|}
\hline Abbreviation & Meaning \\
\hline$\%$ & percent \\
\hline (-)-strand & negative sense strand \\
\hline (+)-strand & positive sense strand \\
\hline${ }^{\circ} \mathrm{C}$ & degrees Celsius \\
\hline$\mu g / \mu L$ & micrograms per microliter \\
\hline$\mu \mathrm{g} / \mathrm{mL}$ & micrograms per millilitre \\
\hline$\mu \mathrm{M}$ & micromolar \\
\hline $3^{\prime}$ & three prime \\
\hline $5^{\prime}$ & five prime \\
\hline aa & amino acid \\
\hline ABCA1 & ABC-transporter A1 \\
\hline ABCG1 & ABC-transporter G1 \\
\hline ABC-transporter & ATP-binding cassette transporter \\
\hline ACAT & Acyl-CoA-Cholesterol-Acyltransferase \\
\hline Alix & programmed cell death 6-interacting protein \\
\hline ALT & alanine-aminotransferase \\
\hline apoA-I & apolipoprotein A-I \\
\hline ApoB100 & apolipoprotein B100 \\
\hline ApoE & apolipoprotein E \\
\hline AU & absorption units \\
\hline BAAT & bile acid-CoA amino acid N-acyltransferase \\
\hline bp & base pairs \\
\hline BSEP & bile salt export pump \\
\hline CaaX & cysteine-aliphatic-aliphatic-variable \\
\hline CARD & C-terminal caspase recruitment domain \\
\hline CD & cluster of differentiation \\
\hline CDK1 & cyclin-dependent kinase 1 \\
\hline cDNA & complementary DNA \\
\hline CGN & cis-Golgi network \\
\hline $\mathrm{CH} 25 \mathrm{H}$ & cholesterol 25-hydroxylase \\
\hline CI & confidence interval \\
\hline cIAP & cellular inhibitor of apoptosis protein \\
\hline CLSM & confocal laser scanning microscopy \\
\hline CMV & Cytomegalovirus \\
\hline CRE & cis-responsive element \\
\hline
\end{tabular}




\begin{tabular}{ll}
\hline C-rich & cysteine-rich \\
\hline CSFV & classical swine fever virus \\
\hline CTCF & corrected total cell fluorescence \\
\hline C-terminal & carboxyterminal \\
\hline CTV & Cutthroat trout virus \\
\hline CYP27A1 & sterol 27-hydroxylase \\
\hline CYP7A1 & cholesterol 7 alpha-hydroxylase \\
\hline CYP7B1 & 25-hydroxycholesterol 7-alpha-hydroxylase \\
\hline DEAD-box & aspartate-glutamate-alanine-aspartate-box \\
\hline DENV & Dengue virus \\
\hline DNA & deoxyribonucleic acid \\
\hline DNM2 & dynamin-2 \\
\hline dNTP & deoxynucleotide triphosphate \\
\hline dpi & days post infection \\
\hline dsRNA & double stranded RNA \\
\hline EC50 & half-maximal effective concentration \\
\hline EE & early endosome \\
\hline EEA1 & early endosome antigen 1 \\
\hline eHEV & quasi-enveloped HEV \\
\hline Em & emission \\
\hline EPDA & End-Point-Dilution-Assay \\
\hline ER & endoplasmic reticulum \\
\hline ERC & endocytic recycling compartment \\
\hline ESCRT & endosomal sorting complexes required for transport \\
\hline Ex & excitation \\
\hline FGF19 & fibroblast growth factor 19 \\
\hline FLAG & FLAG-tag \\
\hline FPP & farnesyl pyrophosphate \\
\hline FXR & farnesoid X receptor \\
\hline FYVE-domain & FYVE zinc finger domain \\
\hline g & gravitational constant \\
\hline g/L & grams per litre \\
\hline GAP & GTPase-activating protein \\
\hline GAPDH & \\
\hline GBP & Glyceraldehyde 3-phosphate dehydrogenase \\
\hline GDP & gandivated site \\
\hline & \\
\hline
\end{tabular}




\begin{tabular}{|c|c|}
\hline GE & genome equivalents \\
\hline GEF & guanine exchange factor \\
\hline GlcNAc & N-Acetylglucosamine \\
\hline GLUE & GRAM-Like Ubiquitin-binding in EAP45 \\
\hline GMP & guanosine monophosphate \\
\hline GOI & gene of interest \\
\hline GRP78 & glucose-regulated protein 78 \\
\hline Gt & genotype \\
\hline GTP & guanosine triphosphate \\
\hline h & hours \\
\hline $\mathrm{HCV}$ & Hepatitis C virus \\
\hline HD & homodimerisation domain \\
\hline HDL & high-density lipoprotein \\
\hline Hel & helicase \\
\hline HEV & Hepatitis E virus \\
\hline HIV & human immunodeficiency virus \\
\hline HMGCR & 3-hydroxy-3-methyl-glutaryl-coenzyme A reductase \\
\hline Hrs & $\begin{array}{l}\text { hepatocyte growth factor-regulated tyrosine kinase } \\
\text { substrate }\end{array}$ \\
\hline HSC70 & heat shock cognate protein 70 \\
\hline HSP90 & heat shock protein 90 \\
\hline HSPG & heparan sulphate proteoglycans \\
\hline HVR & hypervariable region \\
\hline IAV & Influenza A virus \\
\hline ID & interaction domain \\
\hline IDL & intermediate-density lipoprotein \\
\hline IFN & interferon \\
\hline IFNLR1 & IFN- $\lambda$ receptor 1 \\
\hline IFN $\alpha$ & interferon alpha \\
\hline IFNAR & IFN- $\alpha / \beta$ receptor \\
\hline IFN $\beta$ & interferon beta \\
\hline IFN $\gamma$ & interferon gamma \\
\hline IFNGR & IFN- $\gamma$ receptor \\
\hline IgG & immunoglobulin G \\
\hline $\operatorname{IgM}$ & immunoglobulin $\mathrm{M}$ \\
\hline IKK $\varepsilon$ & I-kappa-B kinase-epsilon \\
\hline IL10R2 & interleukin 10 -receptor 2 \\
\hline IL-1R & interleukin-1 receptor \\
\hline
\end{tabular}




\begin{tabular}{|c|c|}
\hline ILV & intralumenal vesicles \\
\hline INSIG1 & Insulin induced gene 1 \\
\hline IRAK & interleukin-1 receptor-associated kinase \\
\hline IRF & interferon regulatory factor \\
\hline ISG & interferon-stimulated genes \\
\hline ISRE & interferon-stimulated response element \\
\hline $\mathrm{IU} / \mathrm{mL}$ & international units per millilitre \\
\hline JAK & Janus kinase \\
\hline JR & junction region \\
\hline $\mathbf{k b}$ & kilobases \\
\hline KSHV & Kaposi's sarcoma associated herpesvirus \\
\hline LAL & lysosomal acid lipase \\
\hline LAMP2 & lysosome-associated membrane glycoprotein 2 \\
\hline LDH & lactate dehydrogenase \\
\hline LDL & low-density lipoprotein \\
\hline LDLR & LDL-receptor \\
\hline LDs & lipid droplets \\
\hline LE & late endosomes \\
\hline LGP2 & laboratory of genetics and physiology 2 \\
\hline LIMP-2 & lysosomal integral membrane protein-2 \\
\hline Luc & luciferase \\
\hline LXR & liver $\mathrm{X}$ receptor \\
\hline Lys & lysosome \\
\hline $\mathbf{M}$ & molar \\
\hline $\mathbf{m}^{7} \mathrm{G}$ & 7-Methylguanosine \\
\hline MAPK & mitogen-activated protein kinase \\
\hline MAVS & mitochondrial antiviral signalling protein \\
\hline MDA5 & melanoma differentiation-associated 5 \\
\hline Met & Methyltransferase \\
\hline Mfn2 & Mitofusin 2 \\
\hline $\min$ & minutes \\
\hline mL & millilitres \\
\hline $\mathbf{m M}$ & millimolar \\
\hline MNV & murine norovirus \\
\hline mRNA & messenger RNA \\
\hline MRP2 & canalicular multispecific organic anion transporter 1 \\
\hline MT & microtubule \\
\hline
\end{tabular}




\begin{tabular}{|c|c|}
\hline MVB & multivesicular bodies \\
\hline Mx1 & interferon-induced GTP-binding protein Mx1 \\
\hline MyD88 & myeloid differentiation primary response 88 \\
\hline NADH & Nicotinamide adenine dinucleotide \\
\hline NEMO & NF-kappa-B essential modulator \\
\hline $\mathrm{NF}-\kappa \mathrm{B}$ & $\begin{array}{l}\text { nuclear factor kappa-light-chain-enhancer of activated } \\
\text { B cells }\end{array}$ \\
\hline $\mathrm{ng} / \mathrm{mL}$ & nanograms per millilitre \\
\hline nHEV & naked capsid HEV \\
\hline NLR & $\begin{array}{l}\text { nucleotide-binding oligomerisation domain-like } \\
\text { receptors }\end{array}$ \\
\hline $\mathbf{n M}$ & nanomolar \\
\hline nm & nanometres \\
\hline NPC & $\begin{array}{l}\text { Niemann-Pick disease, type C1 intracellular } \\
\text { cholesterol transporter }\end{array}$ \\
\hline NTD & nucleotide-binding domain \\
\hline N-terminal & aminoterminal \\
\hline $\mathrm{N}-\mathrm{X}-\mathrm{S} / \mathrm{T}$ & asparagine-variable-serine/threonine \\
\hline OAS1 & 2'-5'-oligoadenylate synthetase 1 \\
\hline OMM & outer mitochondrial membrane \\
\hline ORF & open reading frame \\
\hline ORP1L & oxysterol-binding protein-related protein 1 \\
\hline OST & organic solute transporter subunit \\
\hline p62 & Sequestosome 1 \\
\hline PAMP & pathogen-associated molecular pattern \\
\hline PBC & primary biliary cholangitis \\
\hline PCP & papain-like cysteine protease \\
\hline PCSK9 & proprotein convertase subtilisin/kexin type 9 \\
\hline PEG-IFN & PEGylated Interferon \\
\hline PI3,4,5P3 & phosphatidylinositol 3,4,5-triphosphate \\
\hline PI3,5P2 & phosphatidylinositol 3,5-diphosphate \\
\hline PI3P & phosphatidylinositol 3-phosphate \\
\hline PKR & protein kinase RNA-activated \\
\hline PM & plasma-membrane \\
\hline POI & protein of interest \\
\hline poly-A & polyadenylation \\
\hline pORF & protein encoded by ORF \\
\hline PPAR & peroxisome proliferation-activated receptor \\
\hline
\end{tabular}




\begin{tabular}{|c|c|}
\hline PRR & pathogen-recognition receptors \\
\hline PSAP & proline-alanine-serine-proline \\
\hline qPCR & quantitative polymerase chain reaction \\
\hline $\mathbf{R}^{2}$ & coefficient of determination \\
\hline Rab & ras-related protein \\
\hline Rabex-5 & rabaptin-5-associated exchange factor for Rab5 \\
\hline $\mathbf{R d R p}$ & RNA-dependent RNA-polymerase \\
\hline RE & recycling endosome \\
\hline REP & Rab escort protein \\
\hline RFU & relative fluorescence units \\
\hline RIG-I & retinoic-acid inducible gene I \\
\hline RILP & Rab-interacting lysosomal protein \\
\hline RIPK1 & receptor-interacting serine/threonine-protein kinase 1 \\
\hline RIPK2 & receptor-interacting serine/threonine-protein kinase 2 \\
\hline RLR & retinoic acid-inducible gene-I-like receptors \\
\hline RLU/s & relative light units per second \\
\hline RNA & ribonucleic acid \\
\hline RT-qPCR & reverse transcribed $\mathrm{qPCR}$ \\
\hline RXR & retinoid $X$ receptor \\
\hline s & seconds \\
\hline S1P & site-1-protease \\
\hline S2P & site-2-protease \\
\hline Saps & Saposins \\
\hline SCAP & SREBP cleavage activating protein \\
\hline siRNA & short interfering RNA \\
\hline SNARE & $\begin{array}{l}\text { soluble N-ethylmaleimide-sensitive-factor attachment } \\
\text { receptor }\end{array}$ \\
\hline SRE & sterol-regulatory element \\
\hline SREBP & sterol regulatory element-binding protein \\
\hline ssRNA & single stranded RNA \\
\hline STAT & signal transducer and activator of transcription \\
\hline Syt7 & Synaptotagmin-7 \\
\hline TAK & mitogen-activated protein kinase kinase kinase 7 \\
\hline TANK & $\begin{array}{l}\text { TRAF family member-associated NF-kappa-B } \\
\text { activator }\end{array}$ \\
\hline TBK1 & TANK-binding kinase 1 \\
\hline $\mathrm{TC}$ & total cholesterol \\
\hline TCID $_{50}$ & half maximal tissue culture infection dose \\
\hline
\end{tabular}




\begin{tabular}{ll}
\hline TF & transcription factor \\
\hline TG & triglycerides \\
\hline TGN & trans-Golgi network \\
\hline TGOLN2 & trans-Golgi network integral membrane protein 2 \\
\hline TIR & Toll-interleukin-1 receptor homology \\
\hline TIRAP & TIR domain containing adaptor protein \\
\hline TLR & Toll-like receptors \\
\hline TM & transmembrane \\
\hline tMOC & thresholded Mander's overlap coefficient \\
\hline TNF & tumour necrosis factor \\
\hline TRADD & tumour necrosis factor receptor type 1-associated \\
\hline TRAF & DEATH domain protein \\
\hline TRIF & TNF receptor associated factor 6 \\
\hline TSG101 & TIR-domain-containing adapter-inducing interferon- $\beta$ \\
\hline Tyk2 & tumour susceptibility gene 101 \\
\hline UTR & tyrosine kinase 2 \\
\hline UV & untranslated region \\
\hline v/v & ultraviolet \\
\hline VLDL & volume per volume \\
\hline VLP & very low-density lipoprotein \\
\hline Vps34 & virus-like particle \\
\hline Vps4 & vacuolar protein sorting protein 34 \\
\hline w/v & vacuolar protein sorting-associated protein 4 \\
\hline $\mathbf{w t}$ & weight per volume \\
\hline $\mathbf{X}$ & wild type \\
\hline $\mathbf{X I A P ~}$ & X-domain \\
\hline $\mathbf{Y}$ & x-linked inhibitor of apoptosis protein \\
\hline & Y-domain \\
\hline &
\end{tabular}




\section{Introduction}

\subsection{The Hepatitis E virus}

\subsubsection{Classification and phylogeny}

In 1978, an epidemic of jaundice has been reported in the region of Kashmir, India, which was linked to cases of a non-A, non-B hepatitis [1]. Shortly after, a similar outbreak was observed in a USSR military camp in Afghanistan, which led to first descriptions of virus-like particles (VLPs) extracted from stool samples [2]. As the pathogen carried various features of the Hepatitis A virus, it was classified within the family of Picornaviridae [3]. However, the virus was re-classified several times in the following years, until it was classified as sole member of the Hepeviridae family in 2006 and termed Hepatitis E virus (HEV). This novel family is split into two genera, namely Orthohepevirus and Piscihepevirus [4]. While the latter only comprises one species of the fish-pathogenic Cutthroat trout virus (CTV), the first comprises four species: Orthohepevirus A-D. Herein, Orthohepevirus B-D display viruses only infecting animals, such as bats, birds, ferrets and rats. Solely the biggest species within Orthohepeviruses, Orthohepevirus A, comprises human pathogenic genotypes [5]. These are restricted to genotype 1-2, displaying genotypes only infecting humans, and genotypes 3, 4 and 7, which bear zoonotic potential [6] with genotype 8 being under discussion of causing human infections. Among the eight known genotypes of Orthohepevirus A, various mammals can be found as hosts (Figure 1) [6-8]. 


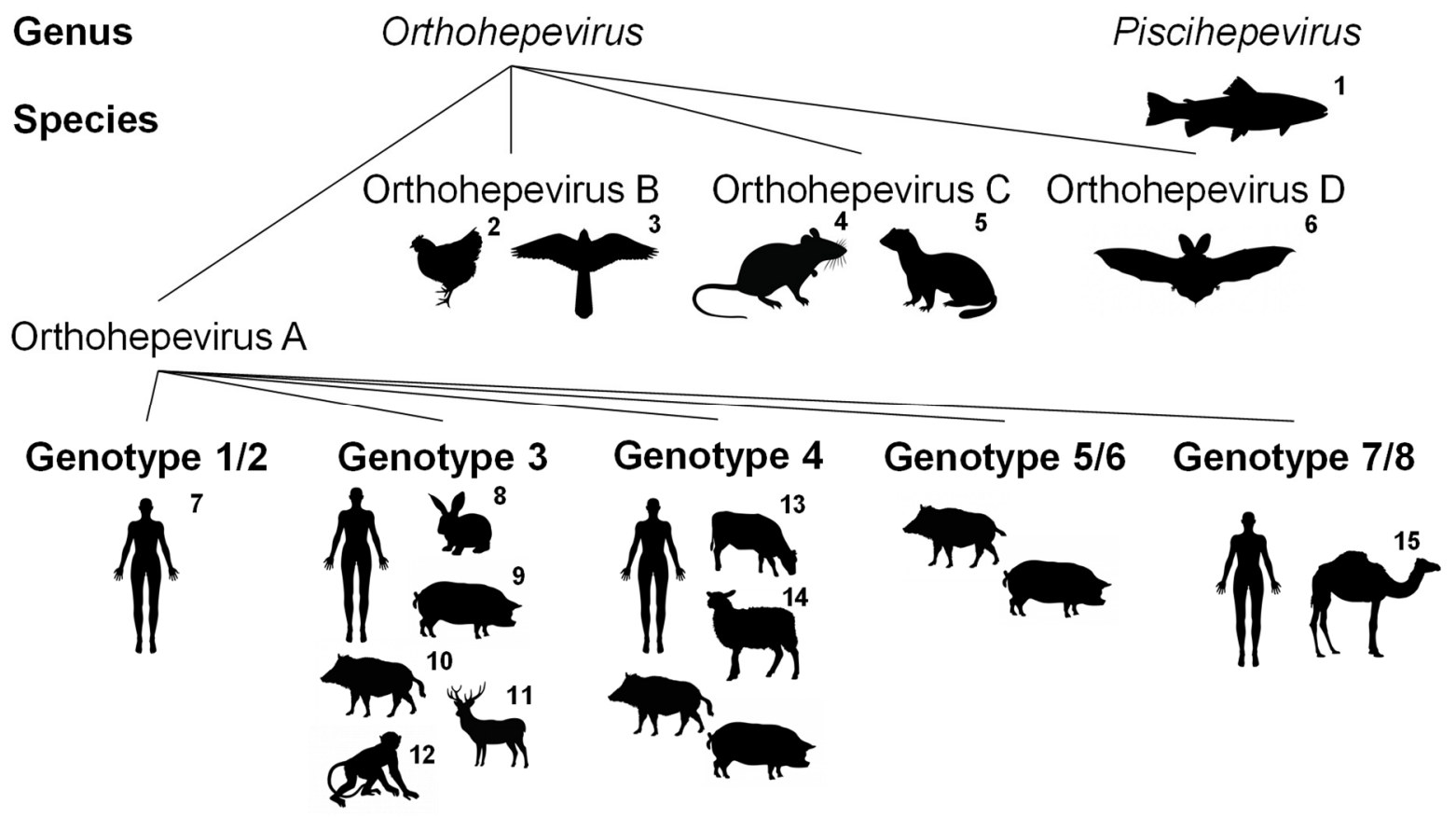

Figure 1. Phylogenetic tree of the family Hepeviridae. While Piscihepeviruses solely target fish, Orthohepeviruses target a variety of different species. Orthohepevirus B-C herein target rodents, birds or bats. Solely Orthohepevirus $\mathrm{A}$ is described to infect bigger mammals, including humans, with genotype 3 and 4 displaying the broadest range of hosts. Silhouettes represent hosts according to the following numbering: ${ }^{1}$ Cutthroat trout, ${ }^{2} \mathrm{chicken},{ }^{3} \mathrm{kestrel},{ }^{4} \mathrm{rat}$, ${ }^{5}$ ferret, ${ }^{6}$ bat, 7 human, ${ }^{8}$ rabbit, ${ }^{9}$ pig, ${ }^{10}$ wild boar, ${ }^{11}$ deer, ${ }^{12}$ primate, ${ }^{13}$ cattle, ${ }^{14}$ sheep and ${ }^{15}$ camelids. Silhouettes were retrieved from stock-image online sources.

\subsubsection{Genomic organisation and viral proteins}

The genome of HEV is composed of a single-stranded, positive-sense RNA sequence, also referred to as (+)-strand ssRNA, which is $7.4 \mathrm{~kb}$ in size [9]. As modification, the genomic RNA carries a 5'-m7G-cap [10] and a $3^{\prime}$-polyadenylation being encoded genomically. Besides the (+)-strand ssRNA genome, a (-)-strand ssRNA intermediate is synthesised during the viral life cycle [11], serving as template for genomic replication [12]. It further serves as template for the third viral transcript, a $2.2 \mathrm{~kb}$ subgenomic (+)-strand ssRNA, which also is $5^{\prime}$-capped and $3^{\prime}$-polyadenylated [13]. The viral genome is flanked by untranslated regions (UTRs) on both the $5^{\prime}$ - and the $3^{\prime}$ end. The first is 23-26 bp in length and is folded as a hairpin-structure, thereby regulating translation initiation. At least two other RNA secondary structures are described to fulfil regulatory functions, both of which represent cis-responsive elements (CREs) [14]. The first resides upstream of the subgenomic RNA-coding portion of the genome, potentially initiating transcription of the subgenome, whereas the second is found in the $3^{\prime}$-UTR. Three classical open reading frames (ORFs) are described as coding sequences for the entirety of Orthohepevirus A genotypes: ORF13. Only for Orthohepevirus A, genotype 1, a fourth ORF (ORF4) [15] is described (Figure 2). As ORF4 is found as alternative reading frame within ORF1 and ORF3 overlaps with ORF2, the gap in coding sequences amidst the viral genome is termed 
junction region. This separation of viral protein templates may serve regulatory purposes, as both pORF1 and pORF4 are translated from the genomic RNA, yet both pORF2 and pORF3 are translated from the subgenomic RNA [13].

\section{$7.4 \mathrm{~kb}$ genomic (+)-ssRNA}

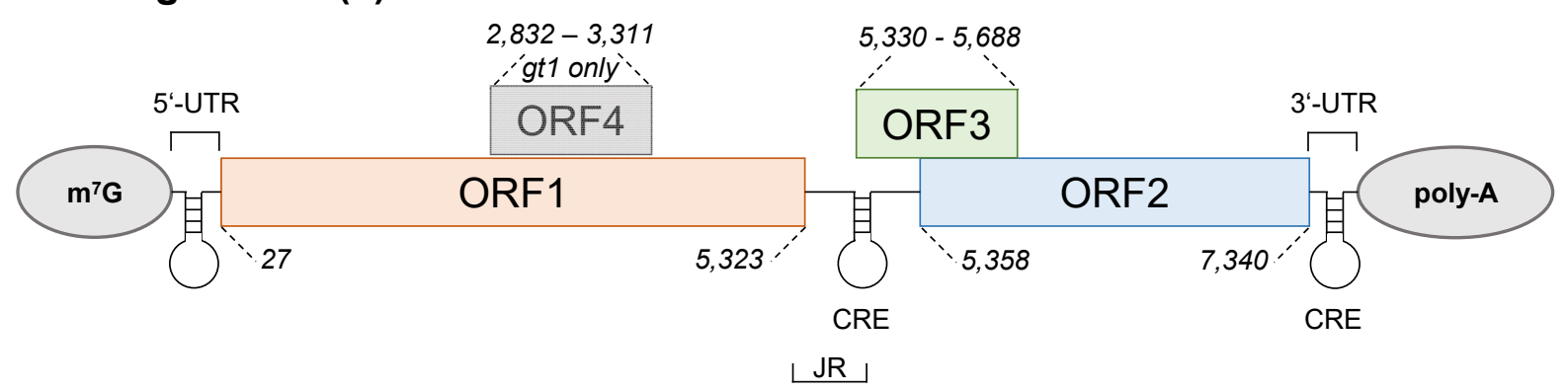

\section{$2.2 \mathrm{~kb}$ subgenomic (+)-ssRNA}

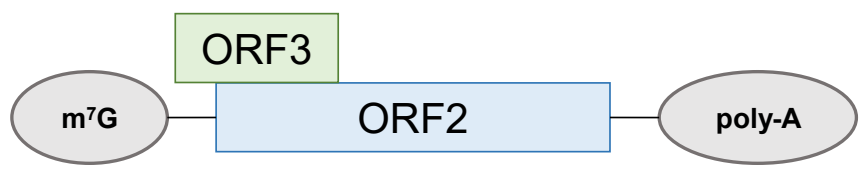

Figure 2. Genomic and subgenomic organisation of HEV. Both the genomic and subgenomic HEV RNA are $5^{\prime}$ capped and 3'-polyadenylated. The genomic RNA encodes the full set of viral proteins via ORF1-4, whereas ORF4 is solely found in Orthohepevirus A genotype 1. Stem-loop structures and cis-responsive elements initiate and regulate RNA-replication and generation of the subgenomic RNA. CRE, cis-responsive element; $\mathrm{m}^{7} \mathrm{G}$, capstructure; poly-A, polyadenylation; ORF1-4, open reading frame 1-4; JR, junction region; UTR, untranslated region; nucleotide positions marked in italics according to HEV genotype 3, isolate 47832c (ORF1-3) or Nair et al., 2016 (ORF4).

The first and largest protein encoded by the HEV genome is pORF1, being 1,765 amino acids (aa) in size. It fulfils the purpose of genomic replication and is translated as polyprotein comprising the following domains: (i) Methyltransferase (Met), (ii) Ydomain (Y), (iii) papain-like cysteine protease (PCP), (iv) hypervariable region (HVR), (v) X-domain (X), (vi) RNA-helicase (Hel) and (vii) RNA-dependent RNA-polymerase (RdRp) [16]. Whether the polyprotein remains intact or is cleaved via host-factors or the viral PCP is under discussion [17-20]. Here, both full-length and cleaved proteins can be detected and similarly retain functionality. What is clear however, is the role of the RdRp domain (mediating synthesis of the viral genome), the Hel domain (unwinding secondary structures) and the Met domain (capping the genomic and subgenomic RNA). Both the X-and the Y-domain are discussed to fulfil regulatory functions during the viral life cycle [21,22], whereas the HVR comprises features proving important for virus adaptation with respect to viral replication [23,24]. Similarly, pORF4 is described to fulfil functions during viral genome replication, yet further elucidation is required [15]. This classifies both pORF1 and pORF4, the mediators of viral replication, as non-structural proteins of HEV.

A classical structural protein of HEV is found in pORF2, which is 660 aa in length. It forms the capsid structure via oligomerisation and mediates RNA-packaging via a positively-charged, N-terminal domain for generation of progeny viruses [25]. 
Isoforms of the protein were identified in the past, being either secreted as monomer or being associated with virion morphogenesis [26]. Due to pORF2 carrying a signal peptide, it is N-glycosylated via the consensus-motif N-X-S/T at several sites, depending on the genotype and strain, within the endoplasmic reticulum [27-29]. This in turn renders a big proportion of the intracellular protein residing perinuclearly with respect to its subcellular localisation [30]. However, only the unglycosylated pORF2, which uses an alternate start-codon, is responsible for building the virion, which is capable of spontaneous self-assembly [31].

A regulatory accessory protein is represented by pORF3, which is 113 aa in size. Initially it was not linked to virion morphogenesis [32], yet in recent times its role herein became evident $[33,34]$. Whether it displays a transmembrane protein is still under discussion, yet a transmembrane helix is predicted based on the primary structure [35]. Interestingly, recent studies suggest that only palmitoylation within a consensus CaaX-motif renders membrane-localisation of pORF3 [36]. A further posttranslational modification of the protein includes phosphorylation via cyclindependent kinase 1 (CDK1) or mitogen-activated protein kinases (MAPKs) [37]. This phosphorylation is suggested be important for interaction with the viral pORF2 [38], thereby impacting virion morphogenesis. Besides the intermolecular interaction, an intramolecular oligomerisation via its C-terminal portion was identified, thereby forming an ion-channel rendering a viroporin-like function of pORF3 [33]. A summary of viral protein domains and post-translational modifications is presented below (Figure 3). 
A

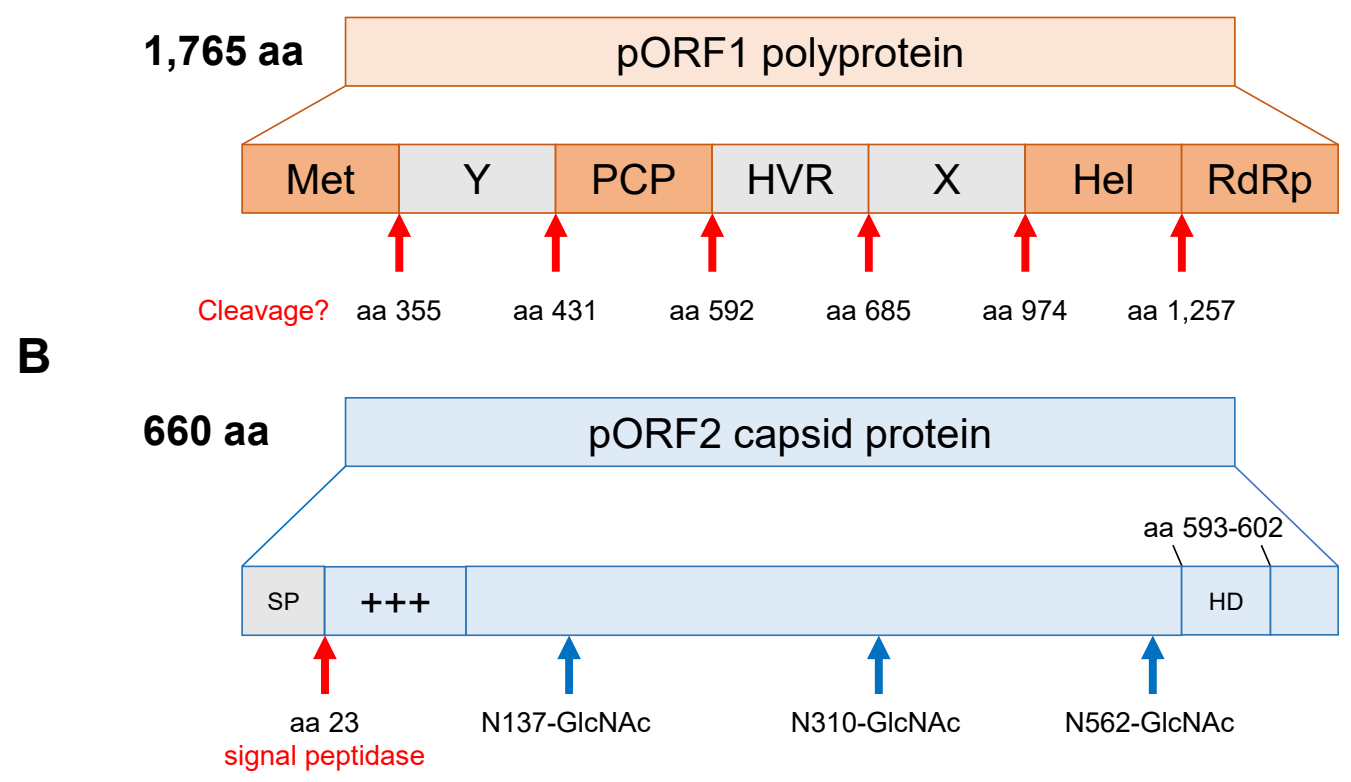

C

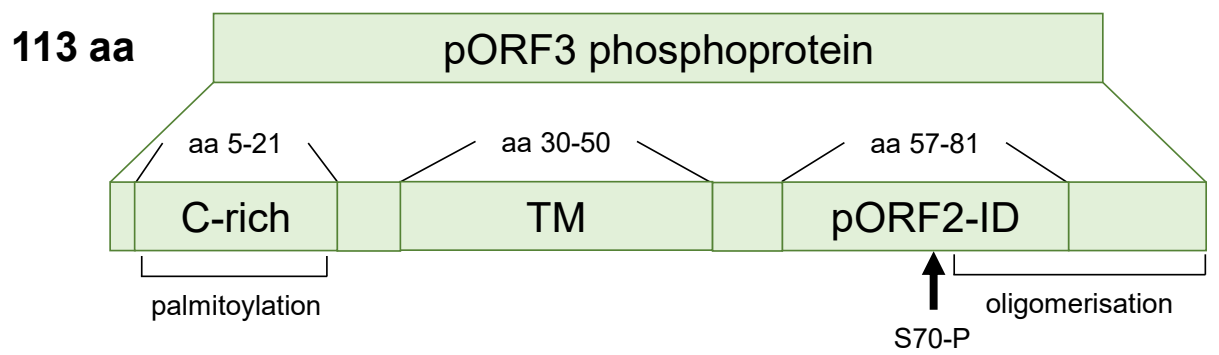

Figure 3. HEV proteins and their functional domains and post-translational modifications. (A) The HEV nonstructural protein pORF1 is a polyprotein divided in several domains. These may be cleaved by proteases to form single, functional or non-functional subunits; non-catalytical domains indicated in grey; potential cleavage-sites indicated by red arrows; Met, methyltransferase; Y, Y-domain; PCP, papain-like cysteine protease; HVR, hypervariable region; X, X-domain; Hel, helicase; RdRp, RNA-dependent RNA-polymerase. (B) The HEV structural protein pORF2 represents the viral capsid protein. Its full form carries a signal-peptide, which is cleaved by the signal-peptidase. Post-translational modifications include N-linked glycosylations at residues N137, N310 and N562; signal peptidase cleavage-site indicated by red arrow; N-linked glycosylations indicated by blue arrows; SP, signal peptide; +++, positively charged stretch; HD, homodimerisation domain; GlcNAc, N-Acetylglucosamine. (C) A regulatory accessory protein is represented by pORF3, which is a small phosphoprotein. It is C-terminally palmitoylated and carries a transmembrane domain. Interaction with pORF2 is dependent on residues 57-81, where the phosphorylation-site at residue S70 is located; phosphorylation-site indicated by black arrow; C-rich, cysteinerich; TM, transmembrane domain; pORF2-ID, pORF2-interacting domain. Amino acid annotations (aa) based on HEV genotype 3, isolate 47832c.

\subsubsection{Transmission and epidemiology}

Transmission of HEV generally occurs via the faecal-oral route as a smear-infection or by ingestion of contaminated material. This is reasoned by the highest concentration of virions found within the stool of infected organisms [2]. Hence, HEV was considered a water-borne virus for an extended period of time, as outbreaks were often linked to e.g. flooded sewers or improper disposal of excrements $[1,39,40]$. However, it became apparent that HEV also can be contracted via consumption of contaminated, 
improperly cooked meat, which in turn is limited to zoonotic genotypes [41,42]. This broadened the perception of HEV representing a food- and water-borne virus. Further transmissions were tracked to originate from blood-transfusions [43,44], while HEV potentially may also be transmitted via copulation, as it is additionally found in ejaculate. A vertical transmission of the virus was detected $[45,46]$, yet is still under discussion to be a major rout of infection [47] (Figure 4).

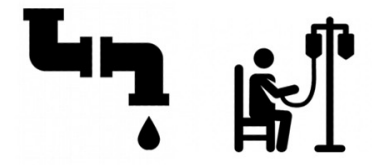

Human sources

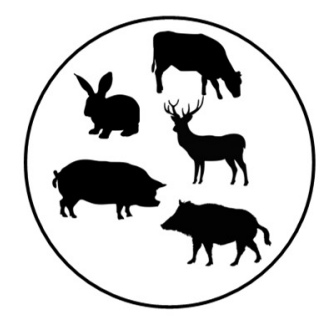

Zoonotic sources
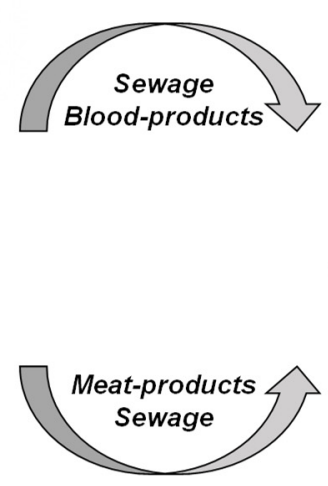

Sewage

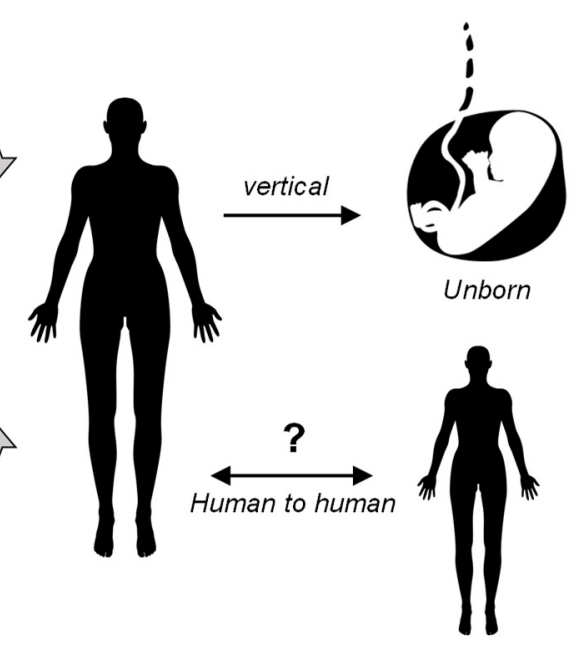

Figure 4. Sources of zoonotic and human-human transmitted HEV-infection. Infections with HEV can be caused via either human sources or animal sources. Human sources include contaminated water, blood-transfusion or vertical spread from mother to child. Animal sources cause a zoonotic transmission via contaminated meat or excrements. Silhouettes were retrieved from stock-image online sources.

First recognised cases of HEV were reported in developmental countries. As they were linked to flooding and poor hygiene standards [48,49], the viral infection was perceived as disease within third and fourth world countries. While it is true that genotype 1 and 2 are mainly found within these regions up until today, zoonotic forms were more and more recognised. The majority of cases contributing to the latter are identified to reside within regions of developed countries, making HEV a worldwide problem [50]. Endemic regions are spread throughout Africa, Asia and Mexico with local outbreaks occurring rather frequently [51,52]. Within these regions, HEV genotype 1 and 2 are predominant. Outbreaks and cases of genotype 3 and 4 in the Americas, Europe, Australia, South-Korea or Japan directly correlate with the occurrence of the respective zoonotic form, swine HEV [53] (Figure 5). The wide distribution of endemic regions and sporadically occurring cases in developed countries make HEV the commonest cause of an acute, viral hepatitis worldwide. In developing countries, an overall seroprevalence of far above $25 \%$ is reported [54], yet also in developed countries seroprevalence can reach as high as $24 \%$, e.g. in central Europe [55]. In the latter, the number of autochthonous cases is detected to be on the rise during the past decade [56]. In summary, a total of over 20 Million cases of an HEV-infection are registered each year $[57,58]$. 


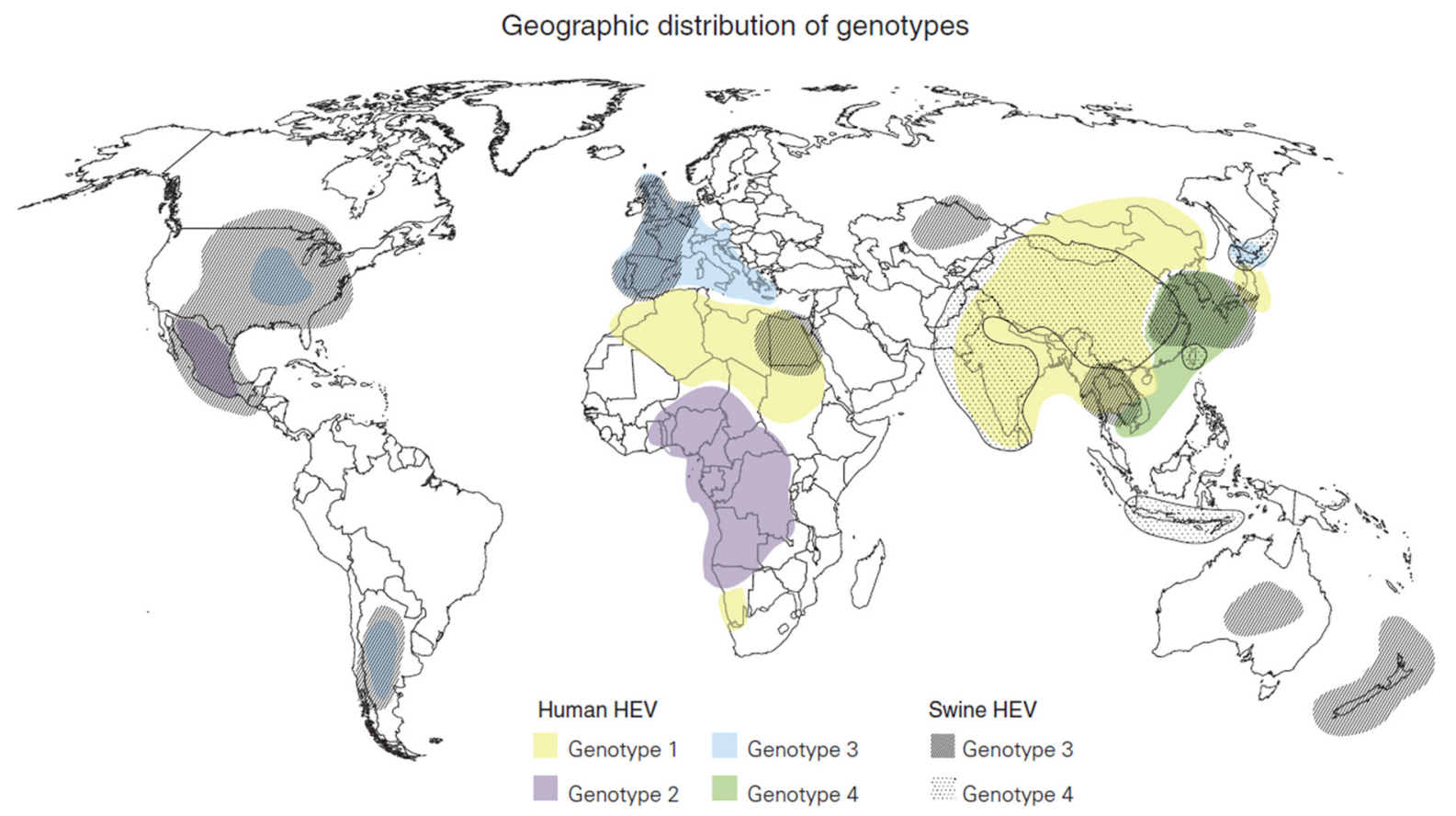

Figure 5. Geographical spread of human-pathogenic HEV. Geographically, Orthohepevirus A genotype 1 and 2 are mainly found in third world countries and Mexico. Zoonotic genotypes 3 and 4 are more wide-spread in Europe, the Americas and Japan, which overlaps with e.g. Swine-HEV as causative agent for zoonotic transmissions. Occurring cases and outbreaks are separated by host. Image retrieved from [59].

\subsubsection{Pathogenesis and treatment}

Upon exposure to HEV, most patients develop no or very mild symptoms. These are developed between 2 and 8 weeks post infection [60]. Initial symptoms of an acute Hepatitis E include malaise, fever and nausea, which can easily be mistaken for other infectious diseases such as a flu or a Hepatitis A. Since an acute HEV-infection often is self-limiting, most patients refrain from consulting a physician. With the disease persisting however, patients start to develop e.g. abdominal pain, hepatomegaly, excretion of pale stool and jaundice. These hepatic symptoms co-occur with the onset of seroconversion and increased alanine-aminotransferase (ALT)-concentrations in the blood, as well as rising viral titres and viral shedding into blood and stool [61-63]. Besides damage to the liver, HEV-infections also bear the risk of extra-hepatic manifestations. Here, a variety of different organs were described to be affected with the majority of cases were found to express damage to the central nervous system or the kidneys [64]. A relief from symptoms conventionally is in place once HEV-IgG levels rise in infected patients, yet some develop fulminant hepatitis potentially being supported by a poor T-cell response [65] or the progesterone-receptor [66]. A partial link between progesterone levels and pathogenesis also was reported for rhesus macaques [67]. This ultimately results in liver and multi-organ failure, leading to the death of the infected individual. Such fulminant cases are mainly described in pregnant women, rendering an overall mortality rate of $\sim 25 \%$ within the latter, 
compared to $0.1-4 \%$ mortality rates in healthy adults $[68,69]$. With respect to these symptoms and the resulting clinical outcome, developing countries do not differ significantly from developed countries [70]. However, a difference between clinical outcomes of an infection with the human-only genotypes (gt1 and gt2) and the zoonotic genotypes (gt3, gt4 and gt7) is evident. While all of these genotypes mainly cause an acute hepatitis, fulminant courses are only reported for the first [71-73], whereas chronification was only reported for the latter [74-76]. Chronification of an HEV-infection is mainly triggered by external sources and underlying diseases. In essence, an impaired immune-response causes the infection to persist and subsequently be converted from an acute course to a chronic course. Immunodeficiency or immunosuppression herein is caused by either an infection with human immunodeficiency virus (HIV) or treatment with immunomodulators during solid organ transplant [77-80]. Taken together, self-limiting, fulminant and chronic HEV-infections lead to the death of up to 44,000 individuals per year [81,82]. A general overview of the clinical course of an HEV-infection is presented below (Figure 6).

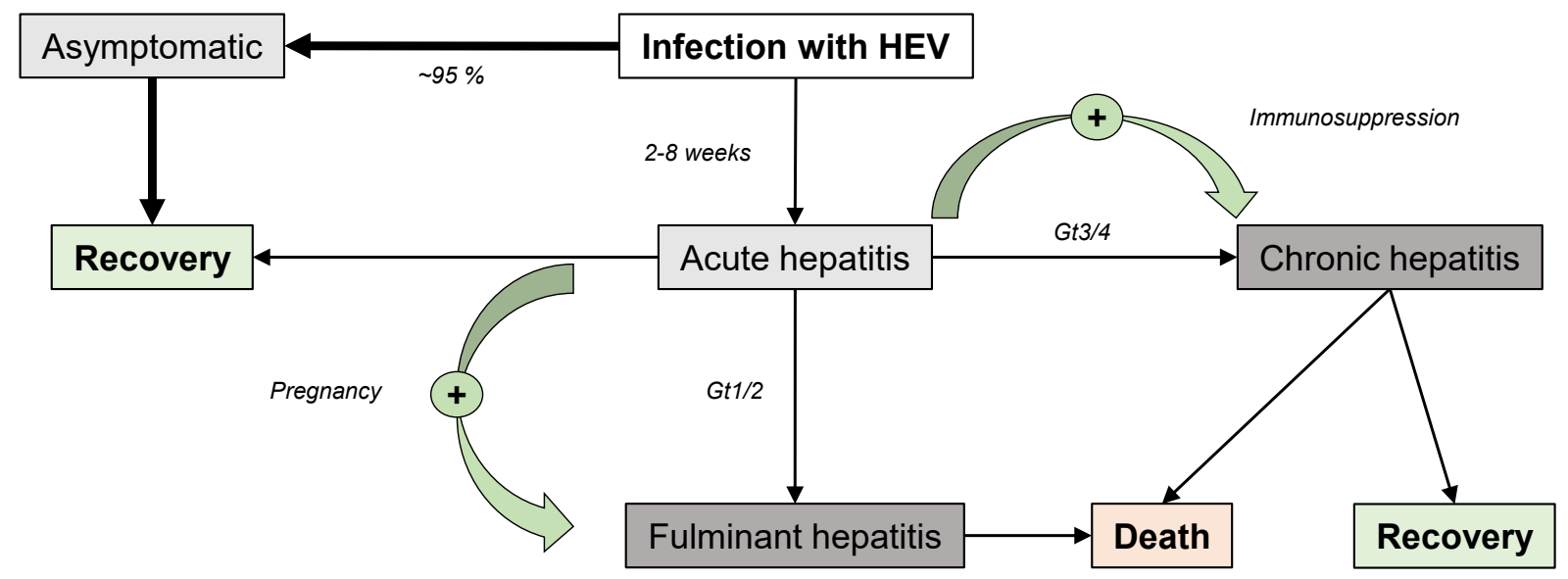

Figure 6. Clinical outcomes of an HEV-infection. Upon infection, $95 \%$ of patients develop no symptoms and recover well. If symptoms occur, this takes place 2-8 weeks after infection leading to an acute hepatitis. Especially in pregnant women infected with genotype 1 or 2 , a fulminant hepatitis may develop leading to death. An acute hepatitis based on an infection with genotype 3 or 4 majorly in immunosuppressed patients leads to chronification leading to either death or recovery. Gt1/2, Orthohepevirus A genotype 1 or 2; Gt3/4, Orthohepevirus A genotype 3 or 4 .

The majority of HEV-infections being treated is represented by chronic infections. This is due to the viral infection being self-limiting most times or leading to death during fulminant courses rather quickly. As the virus itself is tremendously understudied, treatment options are sparse although HEV represents an uprising thread to industrialised countries. A broad-spectrum antiviral is applied under use of PEGylated Interferon alpha (PEG-IFN), which induces the host immune system to rid the body from infected cells. However, this treatment is linked with severe side-effects, as it is a non-targeted form of therapy and is therefore not suitable for a wide range of patients [83-85]. A further treatment option is represented by the use of Ribavirin, a 
nucleoside analogue also being used to treat e.g. Hepatitis $\mathrm{C}$ virus (HCV) infections $[86,87]$. While this treatment option induces less adverse effects, the use of it is not unproblematic as it may trigger drug-resistance of HEV. Specifically, a mutation acquired in the viral $\operatorname{RdRp}(\mathrm{G1634R})$ renders Ribavirin to lose its antiviral activity [88]. Thus, there is an urgent need of alternative medication to treat the viral hepatitis. Progress in this was achieved by using the plant extract silvestrol [89] or a related compound [90], which are currently in pre-clinical development.

\subsubsection{Viral life cycle}

The life cycle of HEV, as for all pathogens, starts with cell entry. Although HEV represents a naked capsid virus, two forms of the viral particle exist: (i) naked capsid HEV (nHEV), found in stool and (ii) quasi-enveloped HEV (eHEV), found in sera or cell culture supernatant. As these carry differing sets of surface proteins, entry mechanisms differ just as well. For both, however, initial attachment seems to be facilitated by unspecific interactions with heparan sulphate proteoglycans (HSPGs) [91]. Subsequently, nHEV is internalised under assistance of heat shock cognate protein 70 (HSC70), glucose-regulated protein 78 (GRP78) and heat shock protein 90 (HSP90), ultimately budding into Clathrin-coated pits thereby forming endosomes [92-94]. As this may also partially be true for eHEV, viral entry seems to differ between the two forms of virions. Specifically, a certain requirement of ras-related protein 5 (Rab5) and Rab7 seems to be in place for the entry of eHEV, but not for nHEV [95]. Notably, the entry process is independent from macropinocytosis, yet it requires cholesterol and dynamin-2 (DNM2). Both trafficking of virus-containing endosomes and subsequent RNA-release remain elusive, although this process may partially depend on cholesterol as well [96]. Upon release of the viral genome, the nonstructural polyprotein pORF1 is translated via the host-machinery. Subsequent synthesis of the (-)-strand ssRNA intermediate [11] by the viral RdRp enables formation of both genomic RNA and subgenomic RNA encoding the structural protein pORF2 and the accessory protein pORF3 [13]. The exact subcellular localisation of viral replication remains elusive, although an involvement of compounds of the early secretory pathway is suggested [97].

A similar lack in knowledge is present regarding the place of capsid morphogenesis, yet both the ER and the cis- and trans-Golgi-network may be involved in bringing the RNA in close proximity to pORF2 [98-100]. After incorporation of viral RNA into the capsid, pORF3 is found to interact with PORF2, thereby potentially coating the capsid surface [38]. This proves to be of utmost importance, as pORF3 carries a classical viral late domain, a PSAP-motif, interacting with tumour susceptibility gene 101 (TSG101) [101]. As the latter represents a critical component of the endosomal sorting complexes required for transport (ESCRT), which in turn reside in multivesicular bodies (MVBs), 
pORF3 may aid to recruit the viral capsid to these late endosomes [102]. In fact, once this interaction is disturbed, viral egress reduces dramatically [34,103]. Further, pORF2 is found in association with programmed cell death 6-interacting protein (Alix), vacuolar protein sorting-associated protein 4A (Vps4A) and Vps4B [104]. All of these are involved in ESCRT-dependent formation of intralumenal vesicles (ILVs) in MVBs and imply an incorporation of $\mathrm{HEV}$ virions into these late endosomes. Upon virion invagination, late endosomes are shuttled to the plasma-membrane in a Rab27dependent manner [105]. Ultimately, HEV virions are released from infected cells while still being incorporated in ILV-originating membranes. These represent the source of the viral envelope, rendering eHEV to carry an exosomal membrane $[100,106]$. Only in an infected organism, this envelope is stripped off the capsid due the latter being exposed to bile salts in the biliary duct. From there, de novo synthesised progeny virions are excreted as nHEV via stool [107] (Figure 7).

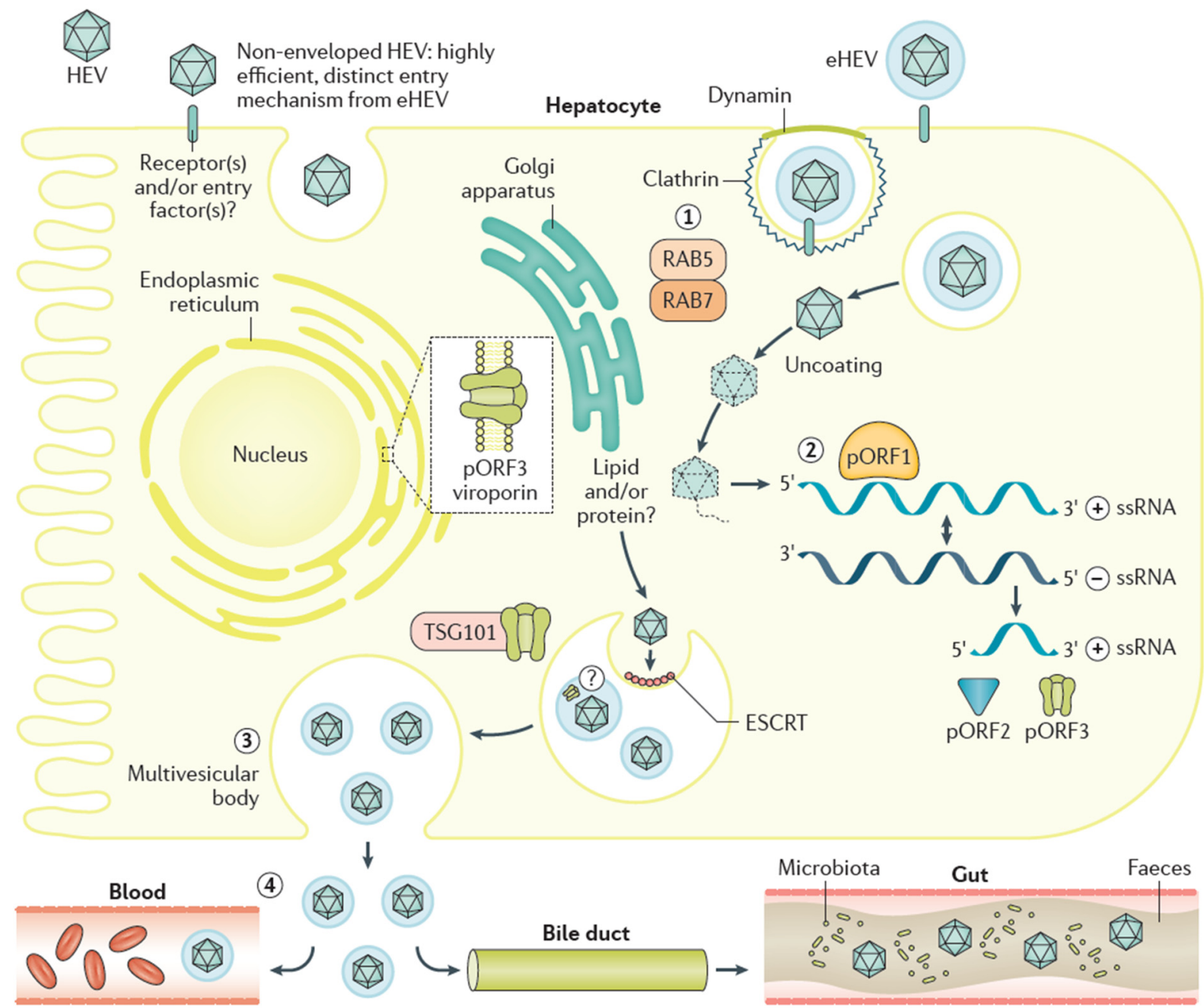

Figure 7. Overview of the HEV life cycle. (1) Virus internalisation and RNA uncoating via clathrin-coated pits and DNM2 under modulation of Rab5 and Rab7. (2) pORF1-dependent genomic replication, synthesis of subgenomic RNA and translation of viral proteins. (3) Release of progeny virus via MVBs in a TSG101-pORF3-dependent manner as exosomes from infected cells. (4) Shedding of progeny virus into blood and bile duct, with the latter representing the place of removal of the viral quasi-envelope. Image retrieved from [108]. 


\subsection{Non-systemic innate immunity}

\subsubsection{Pathogen-recognition receptors in viral infections}

Several key-regulators in cell-specific immune response have been identified in the past years. These differ from pathogen to pathogen, each one being recognised by a set of receptors. Generally, a motif being recognised by so called pathogen-recognition receptors (PRRs) is termed pathogen-associated molecular pattern (PAMP), which can be protein, lipid or nucleic acid. One major class of PAMPs being sensed within infected cells is represented by the viral genome. As HEV represents a single-stranded RNA virus, there are three major classes of PRRs involved in sensing of a viral infection within the infected cell: (i) Toll-like receptors (TLRs), (ii) retinoic acid-inducible geneI-like receptors (RIG-I-like receptors, RLRs) or (iii) nucleotide-binding oligomerisation domain-like receptors (NOD-like receptors, NLRs). Each of these triggers unique signalling cascades, ultimately leading to induction in gene-expression of e.g. inflammatory cytokines or interferons.

Viruses are approached by TLRs upon binding to the target cell, subsequent virus internalisation or progeny virus being present in endosomes. These class I transmembrane proteins, homologues to the Toll-receptor in Drosophila, subside in both plasma membrane and endosomes [109]. TLR2 and TLR4 represent molecules being responsible for sensing of viral proteins on the cell surface and are subsequently internalised [110,111]. On the other hand, TLR3 and TLR7-9 mainly reside in the endosomal system, herein recognising viral nucleic acids [112-115]. A feature shared by all TLRs is the C-terminal, intracellular Toll-interleukin-1 receptor (IL-1R) homology (TIR) effector domain [116]. This functional domain is responsible for signal transduction and recruitment of accessory factors, which elicit downstream-signalling. A shared effector and key-modulator of the subsequent cascade is the myeloid differentiation primary response 88 (MyD88) [117], which for TLR2 and TLR4, is engaged with the help of TIR domain-containing adaptor protein (TIRAP) [118]. Subsequently, interleukin-1 receptor-associated kinases (IRAKs) are recruited to the receptor-complex [119], activating the E3 ubiquitin-ligase TNF receptor associated factor 6 (TRAF6), among other TRAFs. The latter process is similarly achieved by TLR3 via a MyD88-independent mechanism, namely by engagement of TIR-domaincontaining adapter-inducing interferon- $\beta$ (TRIF) [120]. As a consequence, TRAF6 activates interferon regulatory factor 7 (IRF7) [121], thereby stimulating interferonproduction. Further, it induces gene-expression of inflammatory cytokines through a mitogen-activated protein kinase kinase kinase 7 (TAK1)-assisted activation of the nuclear factor kappa-light-chain-enhancer of activated B cells (NF- $\kappa B$ ) [122] (Figure 8). 
A similar activation of NF- $\kappa \mathrm{B}$-driven gene-expression is the sensing of an infection via NLRs. Just as some TLRs, NLRs represent intracellular PRRs, which promote targetbinding through a leucine-rich domain. The effector domain shared by all NLRs is defined by a NACHT domain, which induces oligomerisation upon target-binding [123]. Once a target is bound and NLR-oligomerisation occurred, receptor-interacting serine/threonine-protein kinase 2 (RIPK2) is activated under localisation to the NLRresiding C-terminal caspase recruitment domain (CARD) [124]. The process is assisted by cellular inhibitor of apoptosis protein 1 and 2 (cIAP1/2) and x-linked inhibitor of apoptosis protein (XIAP) [125-127]. This in turn leads to a TRAF-dependent activation of NF- $\kappa B$ and subsequent expression of inflammatory cytokines [128].

Both the TLR- and NLR-mediated sensing of viral infections mainly leads to the induction of inflammatory cytokines such as interleukin-1 (IL1), IL12, IL18 or tumour necrosis factor alpha (TNF $\alpha$ ). Nevertheless, both groups of PRRs also partially induce the production of type I interferons, namely interferon alpha (IFN $\alpha$ ) and interferon beta (IFN $\beta$ ). This is, in parts, achieved via modulation of signalling exerted by the third major PRR family, namely RLRs. This group of cytoplasmic PRRs comprises three major members: (i) RIG-I, (ii) melanoma differentiation-associated 5 (MDA5) and (iii) laboratory of genetics and physiology 2 (LGP2). The first mainly recognises singlestranded RNA, whereas the two latter mainly recognise double-stranded RNA, all of which display an oligonucleotide chain-length dependency [129-132]. In general, RLRs represent DEAD-box RNA helicases, whereas substrate-specificity is conferred via the protein's C-terminal domain [133] and therefore serve as a more virus-specific PRR as compared to TLRs or NLRs. Upon substrate-binding, RLRs interact with the mitochondrial antiviral signalling protein (MAVS), residing on the cytosolic interface of mitochondria with the interaction being mediated via respective CARD-domains $[134,135]$. Subsequently, MAVS aggregates on the outer mitochondrial membrane (OMM) and triggers recruitment of TRAF3, the I-kappa-B kinase-epsilon/TANKbinding kinase 1 (IKK $\varepsilon / T B K 1$ ) complex, TRAF family member-associated NF-kappa-B activator (TANK) and NF-kappa-B essential modulator (NEMO). In consequence, this kinase-complex activates both IRF3 and IRF7, which induces the production of type I and type II interferons.

This complex sensing of PAMPS allows host-cells to detect viral infections via several different mechanisms, therefore ensuring host-defence against pathogens. Various different factors are involved to maintain efficient downstream-signalling. Ultimately, this leads to stimulation of the innate immune-system, but most importantly to the onset of internal defence-mechanisms being exerted e.g. via interferons (Figure 8). 


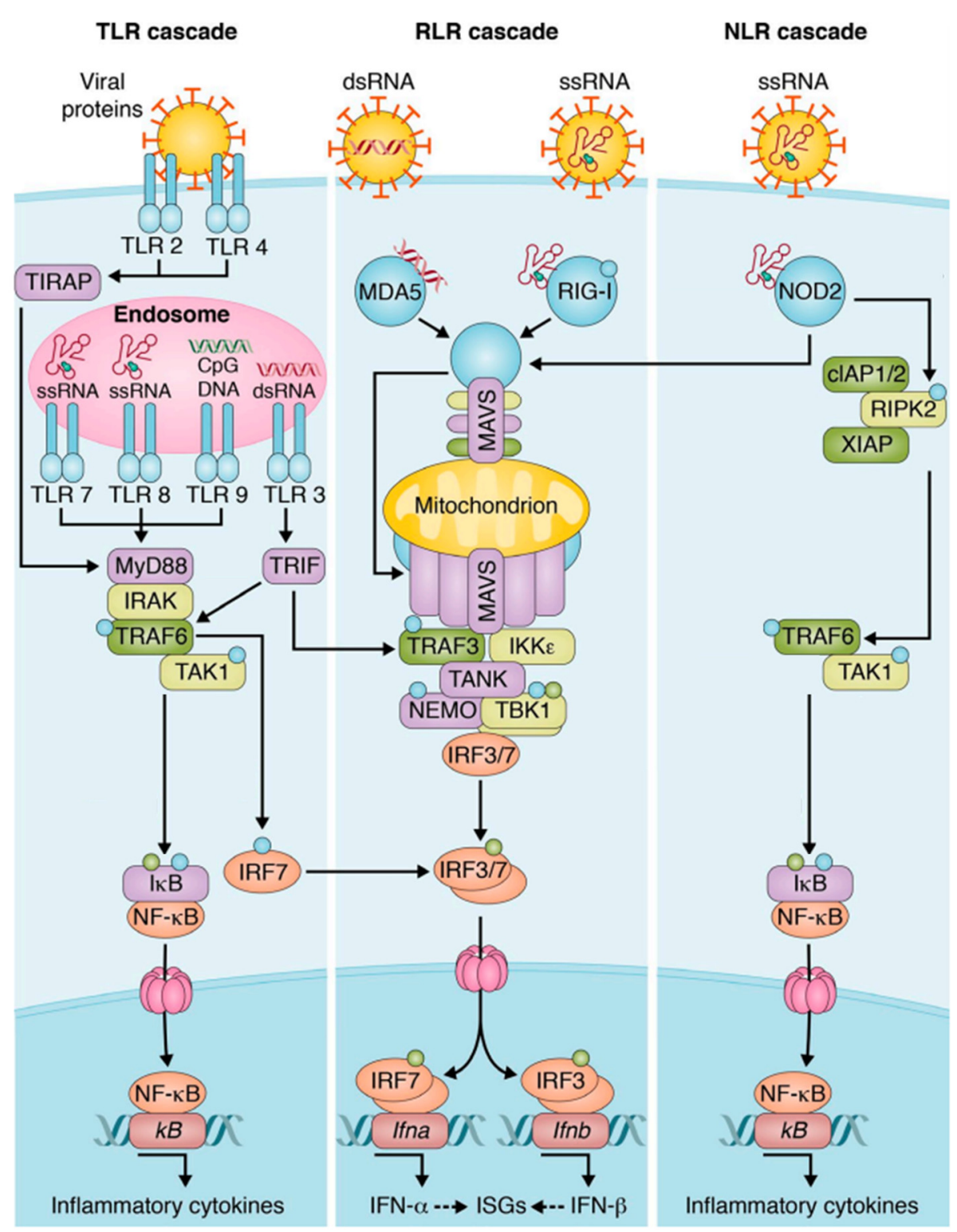

Figure 8. Pathogen-recognition receptor-mediated sensing of RNA viruses and downstream signalling. TLRmediated PAMP-sensing leads to the activation of the MyD88-IRAK-TRAF6-TAK1 complex. Subsequently, this leads to enhancement of NF-kB-induced gene-expression of inflammatory cytokines and to an IRF7-dependent induction of IFN-production. RLR-mediated sensing of PAMPs results in aggregation of MAVS on the OMM, which activates a kinase-complex affecting IRF3- and IRF7-phosphorylation. Ultimately, this induces expression of IFNs. NLR-mediated sensing of viral RNA, similar as TLR-mediated sensing, leads to an activation of NF- $\kappa B$-induced gene-expression. Causative is the activation of kinases such as RIPK2. Image retrieved and modified from [136].

\subsubsection{Cellular responses to interferons}

Three classes of interferons (IFNs) are described in humans: (i) type I IFNs, including IFN $\alpha, \beta, \omega, \kappa, \varepsilon, \delta$ and $\tau$, (ii) type II IFNs, solely including IFN $\gamma$ and (iii) type III IFNs, including different forms of IFN $\lambda$. These IFNs are separated by the type of receptor they are recognised by, with type I IFNs binding IFN- $\alpha / \beta$ receptor 1 and 2 (IFNAR1/2), type II IFNs binding IFN- $\gamma$ receptor 1 and 2 (IFNGR1/2) and type III IFNs binding interleukin 10-receptor 2 (IL10R2) and IFN- $\lambda$ receptor 1 (IFNLR1). Each of these 
receptors is formed via a heterodimerisation of respective monomers once a complex is formed with its ligand $[137,138]$. Depending on the type of activated receptor and thereby on the interacting ligand, different sets of factors are co-activated at the intracellular domain of the receptor-complexes. These factors include Janus kinase 1 (JAK1), JAK2 and tyrosine kinase 2 (Tyk2), which are associated with respective receptors. Once a receptor-heterodimerisation occurs, the kinases are forced into close proximity of each-other through conformational changes and thereby phosphorylate tyrosine residues of one another, as well as the receptor itself [139-141]. In turn, this phosphorylation-dependent activation recruits further factors such as signal transducer and activator of transcription 1 (STAT1) and/or STAT2. Upon phosphorylation by the receptor-complex, these signal transducers are imported into the nucleus. A STAT1-STAT2-heterodimer requires further association of IRF9 to achieve this translocation. Solely in case of an IFN $\gamma$-mediated signalling, STAT3 can be involved in forming a heterodimer with STAT1 [142-145]. This signalling-cascade is termed the Jak-STAT-pathway and efficiently leads to transcription of interferonstimulated genes (ISGs) via activation of the interferon-stimulated response element (ISRE) or the gamma-activated site (GAS) (Figure 9).

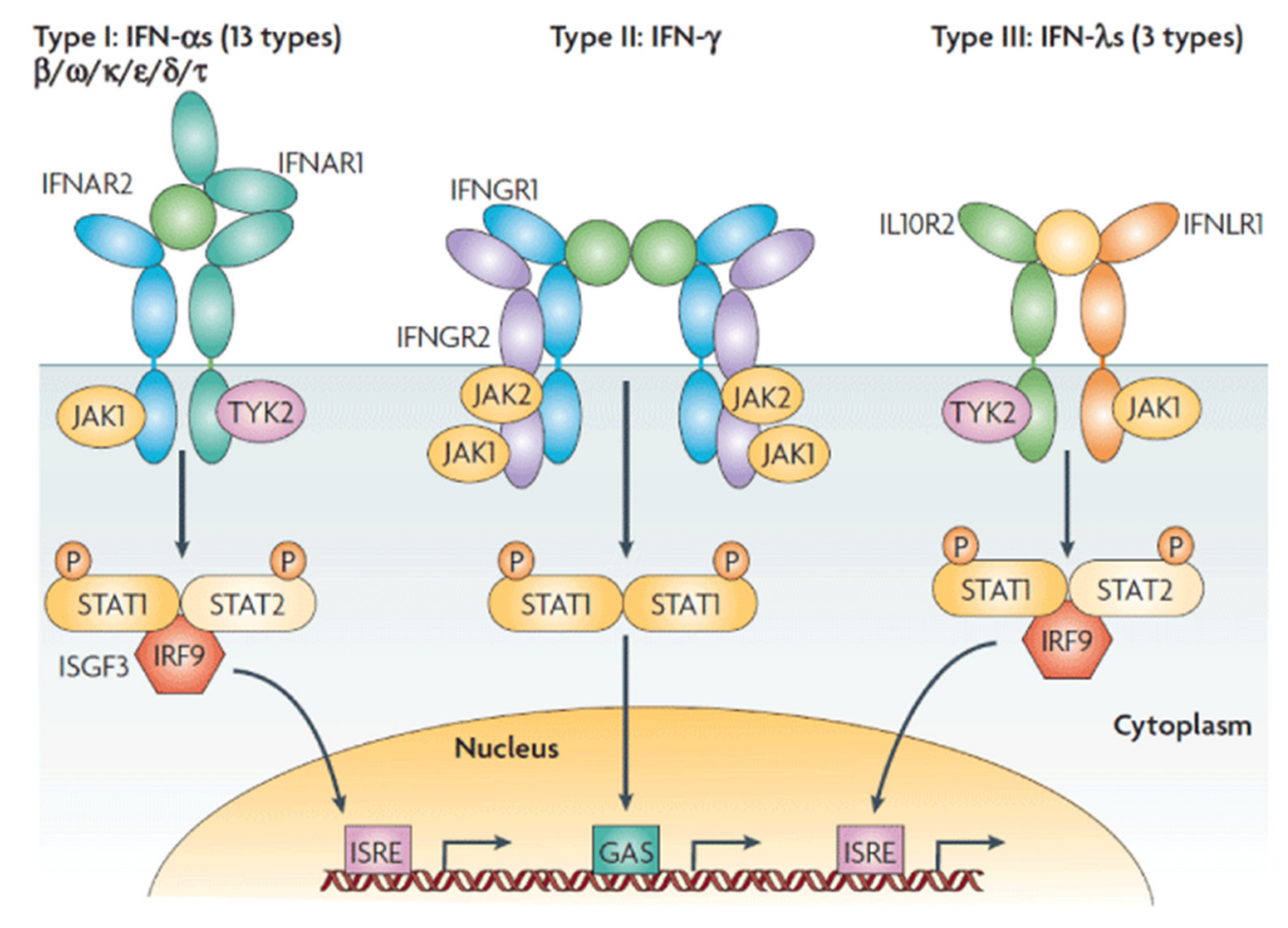

Figure 9. Type I-III IFN signalling pathways for transcriptional regulation. Different IFNs are ligands to different sets of IFNRs. Type I IFNs use the IFNAR1-IFNAR2-dimer to activate STAT1-STAT2 dimers via JAK1- and TYK2dependent phosphorylation. Type II IFNs make use of an IFNGR1-IFNGR2-dimer as receptor, leading to STAT1homodimer activation via phosphorylation through JAK1 and JAK2. Type III IFNs bind a dimer composed of IL10R2 and IFNLR1, thereby triggering TYK2- and JAK1-phosphorylation, which leads to activation of STAT1STAT2-heterodimers. Effectively, Type I and III IFNs induce ISRE promoter elements, whereas Type II IFNS induce GAS promoter elements. Image retrieved from [146]. 
The induction of both ISRE and GAS leads to the expression of manifold different ISGs. Similarly complex are the antiviral effects exerted by these, as they affect virtually all aspects of cellular processes. This in turn mostly renders all steps of viral life cycles to be affected by the IFN-response. There are only few reports describing ISGs for playing a role in restricting an HEV-infection apart from genes also being found in inducing innate sensing or promoting IFN-receptor signalling. However, the extracellular cytokine ISG15 was identified to play a potential role in orchestrating antiviral effects [147]. These are exerted via the 2'-5'-oligoadenylate synthetase 1 (OAS1)-mediated dsRNA-degradation [148], the GTPase interferon-induced GTP-binding protein Mx1 (Mx1) [149] or the protein kinase RNA-activated (PKR)-mediated inhibition of protein translation [150]. Further studies suggest a general, broad-spectrum antiviral activity of IFNs against HEV, yet exact mechanisms or effectors were never studied or identified in detail [151-154].

\subsubsection{Sensing of HEV and viral innate immune-evasion}

Throughout the entire HEV life cycle, several key steps were described to modulate innate immunity. Two major PRRs sense HEV: (i) TLRs and (ii) RLRs. In cell culture, TLRs have been described as restrictive factors for HEV replication, whereas an interference with downstream-signalling partially led to a rescue of HEV $[155,156]$. Major proteins in these cascades were identified as MyD88 and TRIF molecules [155]. One consequence of a TLR-mediated sensing of an HEV-infection, is the elevated production of interferon (IFN)- $\beta$ and $-\gamma[157,158]$. Similar to TLRs, RLRs were described to induce IFN-production through different IRFs in an MAVS/MDA5dependent manner in the context of an HEV-infection [159-161]. Here, certain patterns within the 3'-UTR of the genomic RNA serve as efficient inducer of type I or III IFNinducers depending on the host cell type being exposed to the virus [162].

Opposing to this, the virus itself can modulate a host-cell's IFN-inducing mechanism by various modes of action. The virus' pORF1 PCP-, Met- and X-domain can inhibit IFN type I production via interfering with IRF3- and STAT1-mediated gene-expression $[163,164]$. A more generalised effect was observed for pORF2, as it inhibits NF- $\kappa$ Bresponse by lowering $\mathrm{I} \kappa \mathrm{B} \alpha$ ubiquitination [165], thereby affecting also inflammatory cytokine-production. At last, the regulatory phosphoprotein pORF3 effectively shows a similar interference with STAT1-mediated gene-expression as pORF1 [166], as well as with IFN type I production and can, in parts, prohibit successful IFN- $\gamma$ signalling [167]. Furthermore, pORF3 interferes with the TLR3-mediated downstream cascade by targeting both receptor-interacting serine/threonine-protein kinase 1 (RIPK1) and tumour necrosis factor receptor type 1-associated DEATH domain protein (TRADD) [168]. Potential mechanisms sensing HEV and respective immune-evasion strategies are depicted below (Figure 10). 


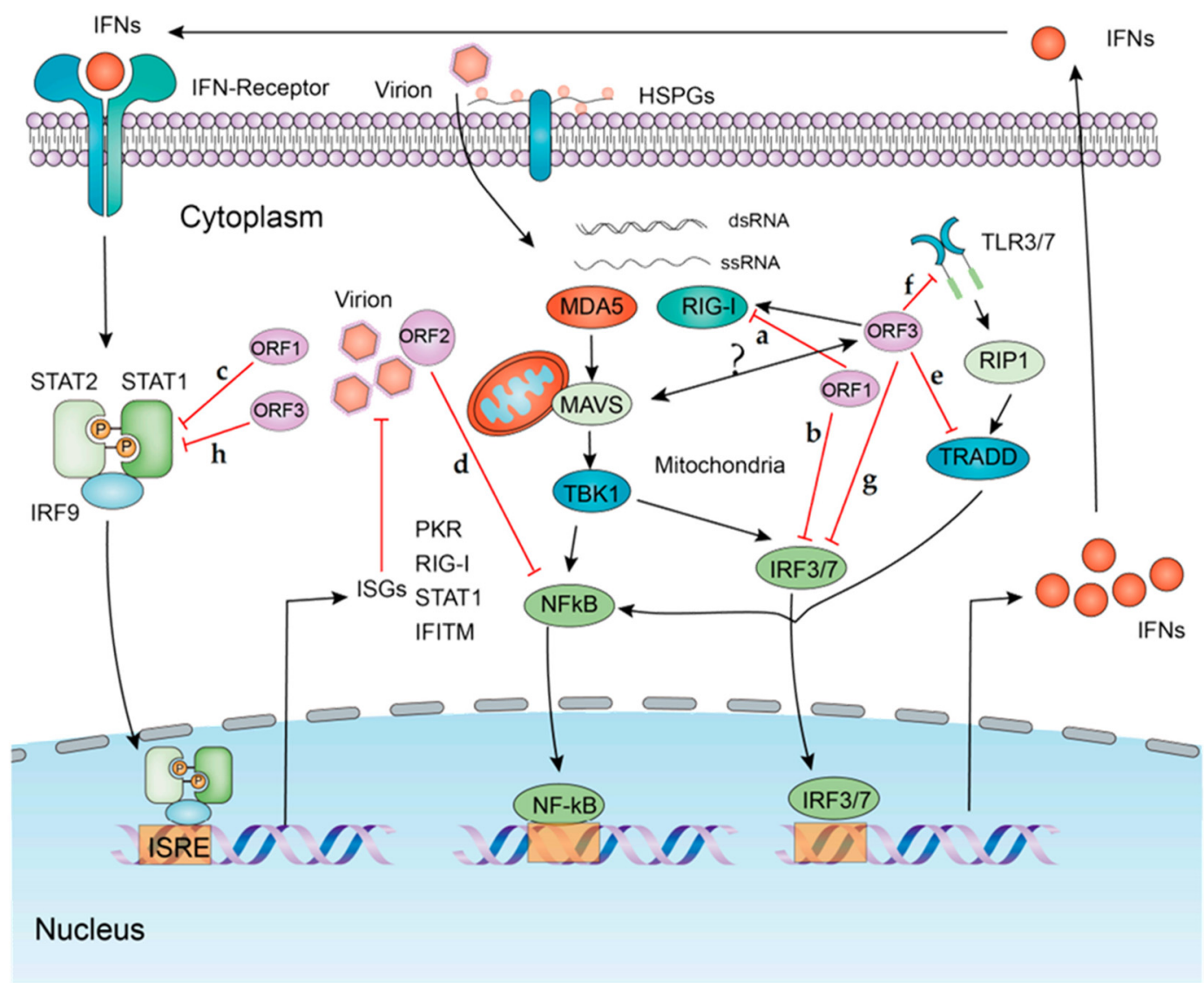

Figure 10. Innate sensing of HEV and virus-related interferences. HEV is mainly sensed through TLR3/7 or the RLRs RIG-I and MDA5. This leads to a TBK1- and TRADD-dependent activation of NF- $\kappa$ B, IRF3 and IRF7, triggering expression of inflammatory cytokines and IFNs. Herein, both pORF1 and pORF3 inhibit RIG-I, TLR3/7 and TRADD (a, e and $\mathbf{f}$ ), thereby interfering with IRF3/7-activation (b and $\mathbf{g}$ ). Further, both viral proteins interfere with STAT-mediated signalling upon IFN-stimulation (c and $\mathbf{h}$ ). Solely pORF2 is described to interfere with expression of inflammatory cytokines by inhibiting NF- $\kappa B(d)$. Image retrieved from [169].

\subsubsection{Guanylate-binding protein 1}

Among heavily induced ISGs upon IFN-stimulation is the family of guanylate-binding proteins (GBPs). Inducibility is achieved by carrying both ISRE and GAS in their respective proximal promoter region, triggering efficient gene-expression [170]. Most prominently, GBP-induction is induced by IFN $\gamma$ [171] and to a lesser extent by type I IFNs [172]. In humans, seven different GBPs are expressed upon IFN-stimulation, which are called GBP1-7 [173]. These proteins have a molecular weight of $\sim 67 \mathrm{kDa}$ and are classified within the dynamin-superfamily. Hence, they comprise both a globular nucleotide-binding domain (NTD) also forming the GTPase-domain [174], as well as an $\alpha$-helical domain necessary to elicit GTP-hydrolysis-driven mechanical effects [175179]. The helical domain can be separated into a helix-bundle following the GTPasedomain, which flows out into a long, paired C-terminal helix interacting with the globular domain [178]. A further feature of GBP1 is a post-translational modification represented by a farnesylation at a conserved CaaX-motif being preceded by a polybasic stretch of amino acids at the C-terminal end of the protein [180]. The 
oligomeric state as well as the membrane-localisation of GBP1 are determined by both its farnesylation and its capacity to hydrolyse GTP to GDP and GMP. Similarly, the oligomeric state affects the efficiency of GTP-hydrolysis. Generally, GBP1 oligomers can only be found upon nucleotide-binding with intermolecular interactions being formed via the globular GTPase-domain [181]. Upon homodimerisation, both the GTPand GDP-bound GBP1 builds up homotetramers [182]. However, the heterodimeric organisation does not require active nucleotide-hydrolysis, as proven by a nonhydrolysing mutant (R48A) still being capable of forming homodimers [175,183]. Effectively, this dimerisation causes a rise in GTP-hydrolysis rates implying important functions for effector-roles of GBP1 [183,184]. In turn, this led to identifications of residues within the protein being important for dimerisation, one of which is represented by serine at position 73 [175]. A further aspect being dependent on the oligomeric state of GBP1 is the post-translational farnesylation. Once dimerisation or oligomerisation occurred, the prenylation causes membrane-localisation of the complex [185], ultimately altering possible protein-protein interactions [186-188] being important for cellular functions. Throughout the nucleotide-binding and hydrolysis steps, GBP1 cycles through different oligomeric states with the long, Cterminal helices undergoing large conformational changes. This gives rise to its dynamin-function [189], which may effectively be used to affect lipid-based structures [185] (Figure 11). 
A

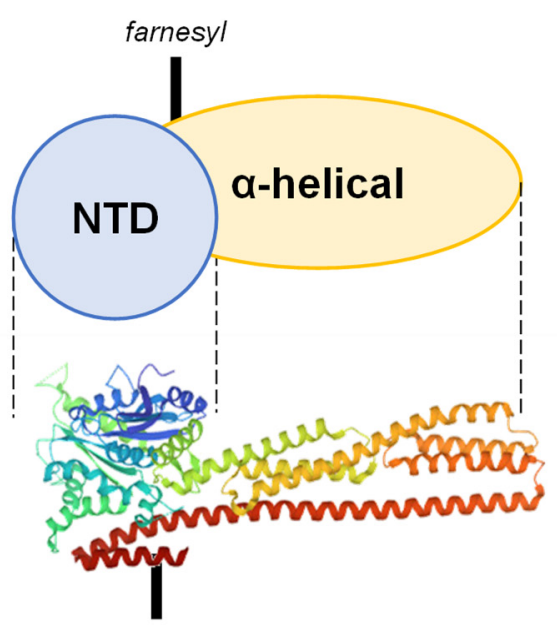

B

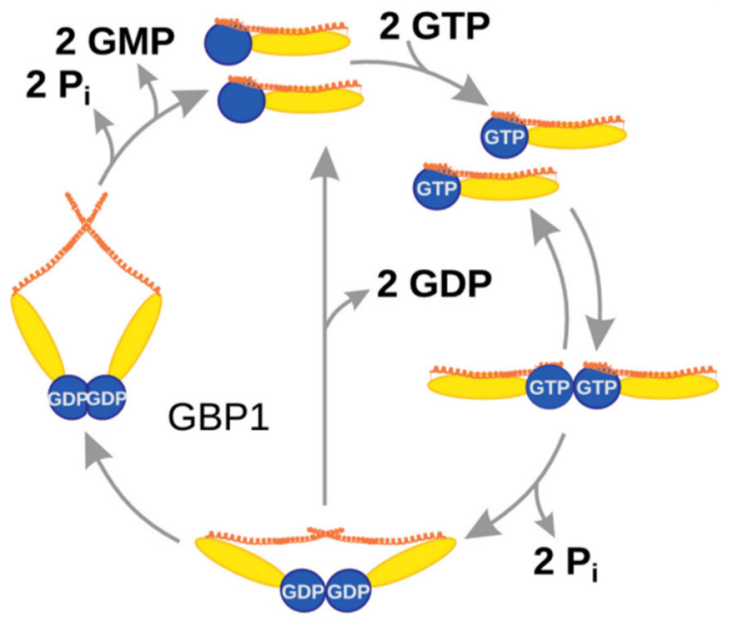

C

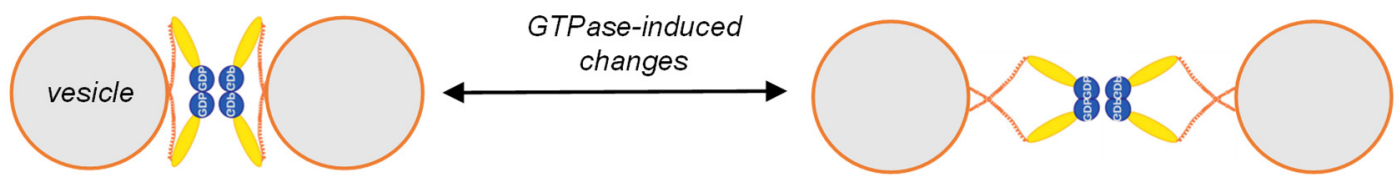

Figure 11. Oligomeric organisation of GBP1 and implications for membrane-targeted effects. (A) GBP1 comprises a large, globular NTD and GTPase-domain, followed by a C-terminal, helical effector-domain harbouring a farnesylation-site. Crystal structure retrieved from [178]. (B) Upon nucleotide-binding, GBP1 homodimerises and cleaves GTP to GDP leading to a small conformational change in the helical domain. Loss of GDP-binding triggers GBP1 homodimers to dissociate and reverts the conformational change. If GDP remains bound, GBP1 further hydrolyses it to GMP, thereby causing a big conformational change. Loss of GMPcomplexation then similarly leads to dissociation of the homodimer and reverts the conformational change. Image retrieved and modified from [189] (C) Farnesylation of GBP1 in the GDP-bound, dimeric state triggers membranelocalisation and/or oligomerisation. Conformational changes induced upon GDP-hydrolysis affect connected membranous structures.

Generally, the GTPase GBP1 is described to act antivirally against a variety of different viruses. Among these are Influenza A virus (IAV) [190], HCV [191], classical swine fever virus (CSFV) [192], Dengue virus (DENV) [193] or Kaposi's sarcoma associated herpesvirus (KSHV) [194]. Here, GBP1 displays the capacity to counteract viral replication in a GTPase-dependent manner [191] or via targeting the cytoskeleton [194,195]. An involvement of GBP1 in other cellular processes such as autophagy was suggested by murine GBPs affecting the degradative process in case of the murine norovirus (MNV) [196] or other pathogens being targeted by GBP1 e.g. via autophagy as described for Toxoplasma gondii [197], chlamydia [198] or mycobacterial infection [199]. Interestingly, no study described the implications of GBP1 and its homodimerisation in the context of a viral infection and endosomal processes.

\subsection{The endolysosomal system}

The endolysosomal system is a complex network of vesicular structures within eukaryotic cells. It orchestrates vesicular trafficking from the extracellular space to intracellular compartments and vice versa. Generally, two sources of cargo are 
described to feed the endosomal system: (i) extracellular components taken up by endocytosis [200,201] or (ii) de novo synthesised components from the ER-TGN network [202,203]. Starting from endocytosis, endosomes gradually maturate [204] while constantly interchanging surface-markers, intralumenal contents and their subcellular localisation in a microtubule (MT)-dependent manner. As a first step, endocytic vesicles are shuttled towards early endosomes (EEs). This in turn represents the first divergence in endosome-trafficking, as cargo can be either passed on to recycling endosomes (REs) being transported back to the plasma-membrane (PM), or further be shuttled towards late endosomes (LEs). Cargo-sorting herein defines the fate of intralumenal contents, which is strictly regulated by sets of manifold different cargo-receptors and intracellular signalling [205,206]. During further maturation, intralumenal vesicles (ILVs) are formed by the central cargo-sorting machinery, ESCRT [207], which gives rise to a population of LEs called MVBs. As a final step of maturation, LEs fuse with lysosomes, thereby forming endolysosomes [208], where cargo is degraded and lysosomes are recycled. Throughout the entire process, backand-forth shuttling of vesicles and proteins is maintained and controlled via the Golgiapparatus, thereby supplying the endosomal system with the required components (Figure 12).

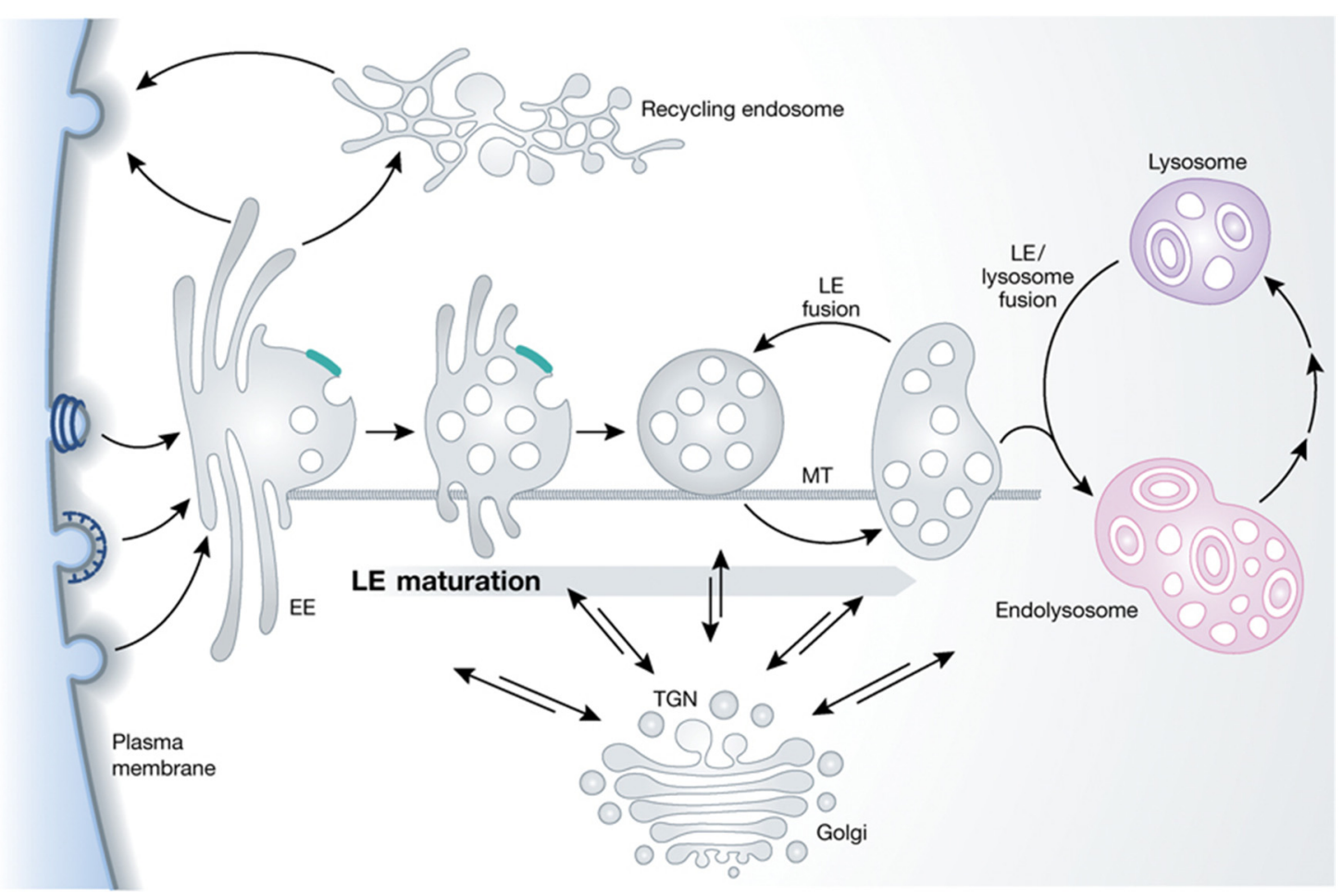

Figure 12. Overview of the endolysosomal system. Upon endocytosis, vesicular structures are transported to EEs. These represent the first sorting step, where cargo is either transported back to the PM via REs or further into the cytosol. EEs gradually mature, while building up ILVs and being shuttled along MTs. A constant supply of components via TGN allows this maturation to happen, ultimately leading to LE-formation. Similarly, endosomal content can be shuttled towards the TGN and be used to supply the cell with extracellular content. A final step in endosome-maturation is represented by the fusion of LEs with lysosomes, thereby forming endolysosomes, where cargo is degraded and lysosomal proteins are being recycled. Image retrieved from [209]. 


\subsubsection{Maturation of endosomes}

The maturation, subcellular localisation and fate of endosomal cargo is highly dependent on three different determinants: (i) vesicle-associated Rab-proteins, (ii) membrane-localised phosphatidylinositols (PI) and (iii) intralumenal decrease in $\mathrm{pH}$ during maturation to LEs.

Rab-proteins represent a family of small GTPases and are key-regulators in vesicular trafficking. Around 70 different types of Rab-proteins were identified, each fulfilling a variety of different roles in affecting vesicular trafficking. Vesicle-association of Rabproteins is achieved via prenylation, rendering them to be peripheral membraneproteins [210]. Generally, Rab-proteins are shuttled to and from their destination by Rab escort proteins (REPs), which directly bind and shuttle freshly prenylated Rabproteins [211]. Once at the destination, Rab-proteins can only fulfil their function while being in a GTP-bound state. This in turn is used to modulate Rab-activity and effectoraffinity via regulation by guanine exchange factors (GEFs), activating Rab-proteins, and GTPase-activating proteins (GAPs), inactivating Rab-proteins [212]. Upon activation, Rab-proteins recruit their effectors, which subsequently fulfil roles in cargobinding, membrane-fusion, vesicular attachment and bidirectional movement along MTs [213]. One well-described example is represented by Rab5 and its effectors. Once Rab5 is exposed on a vesicular membrane of EEs, its respective GEF, rabaptin-5associated exchange factor for Rab5 (Rabex-5), ensures constant supply with GTP [214]. A first effector recruited by Rab5, namely Radaptin-5, enhances Rabex-5 GEFrates, thereby ensuring Rab5 to remain activated [215]. The constant GTP-bound state of Rab5 allows recruitment of a second effector, the phosphatidylinositol 3-kinase vacuolar protein sorting protein 34 (Vps34). The presence of this kinase leads to enrichment of phosphatidylinositol 3-phosphate (PI3P) in the outer membrane leaflet of EEs [216], which in turn is required for further effector-recruitment. These are represented by both the soluble N-ethylmaleimide-sensitive-factor attachment receptor (SNARE)-protein early endosome antigen 1 (EEA1) and Rabenosyn-5, which finally mediate membrane fusion of vesicles with the EEs [217-219] (Figure 13). 

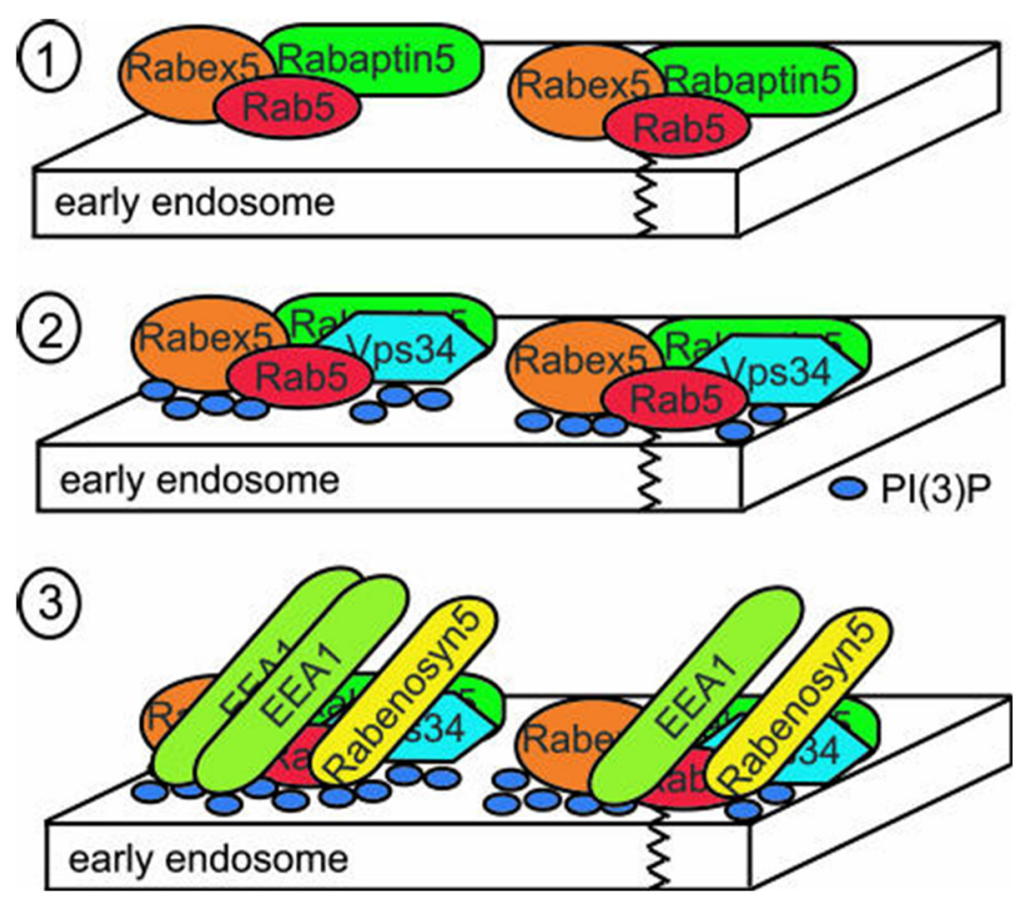

Figure 13. Rab5 and its effectors on the membrane of early endosomes. (1) Upon prenylation, Rab-5 is inserted into membranes of EEs and recruits its GEF Rabex5 and an effector, Radaptin5, to maintain its GTP-bound form. (2) This complex then recruits the PI3-OH-kinase Vps34 as a second Rab5-effector, which phosphorylates PI3-OH and leads to PI3P-buildup on EE membranes. (3) Both presence of Rab5 and PI3P leads to recruitment of EEA1 and Rabenosyn5, which mediate membrane fusion of vesicles with EEs. Image retrieved and modified from [220].

Just as for EEs, Rab-proteins mediated build-up of different PIs via recruitment of various PI-kinases [221]. These in turn recruit further effectors mediating functional effects exerted by the Rab-proteins. Ultimately, this leads to a continuous switch of Rab- and PI-fingerprints of the different sets of endosomes, which can be used to identify different endosomal populations throughout their maturation. While EEs are characterised by the presence of Rab5 and PI3P, recycling endosomes carry Rab4 and Rab35 [222,223]. Rab-conversion with regards to LEs is initiated by exchange to Rab7, leading to build-up of PI3,5P2 [224,225]. On the other hand, EEs exchanging markers to Rab11 results in further build-up of PI3,4,5P3, which then represents the endocytic recycling compartment (ERC) being connected with the TGN network [226,227]. From there on, loss of PI3,4,5P3 leads to fusion with the PM and to release of luminal contents into the extracellular space [228]. Besides the role of Rab-proteins within the endosomal system, they also mediate cargo-import and -export to and from the TGN via Rab6 and Rab9 [229,230] or from ER to the cis-Golgi network (CGN) via Rab1 and Rab2 [231,232]. A fundamental aspect being regulated by these Rab-triggered conversions, besides membrane-fusion, is the targeted transport within the cell guided along MTs. Herein, Rab-proteins recruit distinct effectors representing motor-proteins to maintain retrograde or anterograde vesicular trafficking [233] thereby guiding endosomal content to either being released or being degraded in lysosomes via acid hydrolases activated by the gradual drop in $\mathrm{pH}$ [234] (Figure 14). 


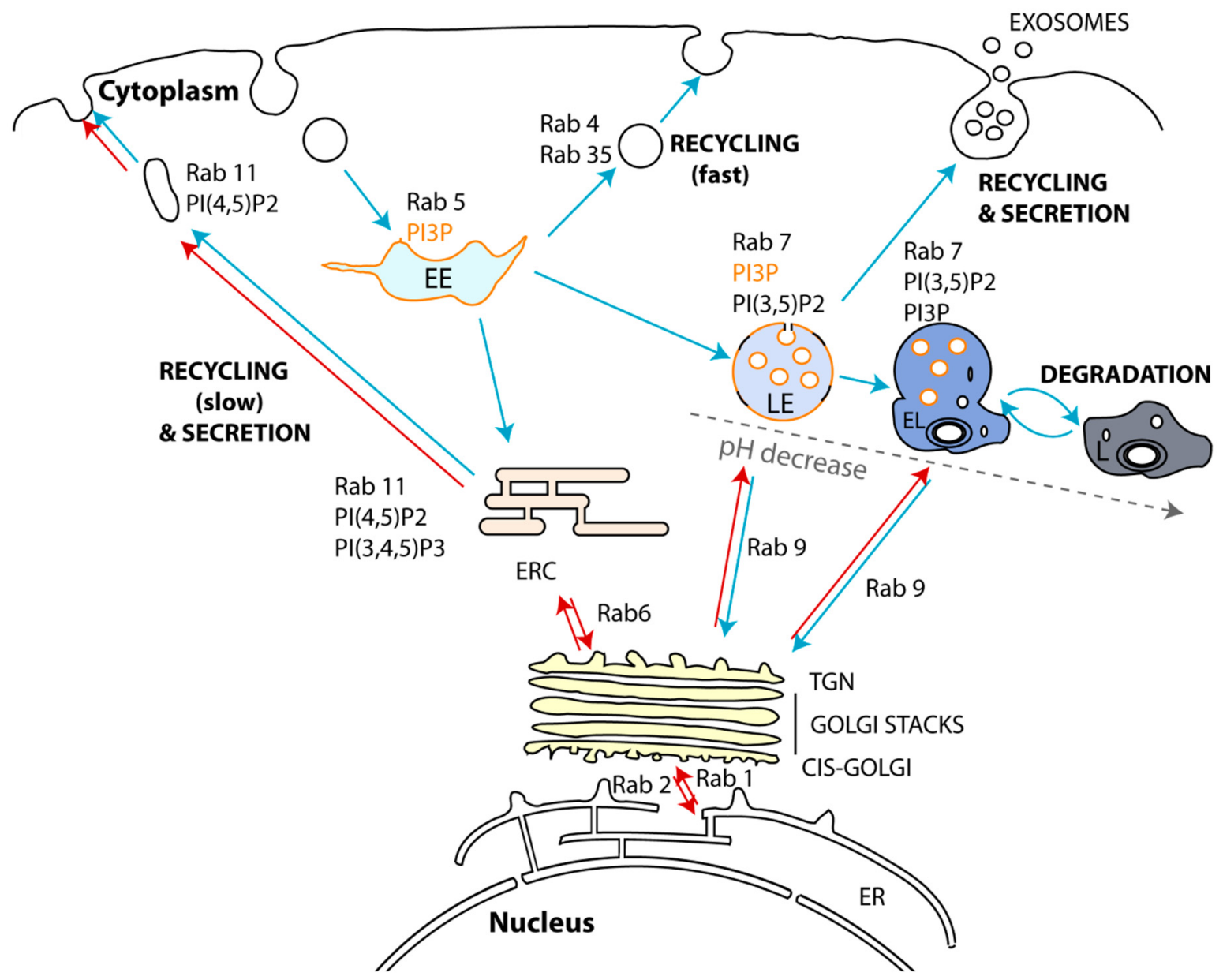

Figure 14. Endosome maturation in dependency of Rab-proteins and phosphatidylinositol. Upon internalisation of cargo, the endosomal system shuttles incorporated components to the required destination. From EEs over LEs to Lysosomes (L), this sorting and maturation is accompanied by conversion of associated Rab-proteins. Similarly, EE-to-ERC shuttling, Golgi-associated shuttling and cargo-recycling is mediated by the small GTPases. This leads to establishment of unique fingerprints of endosomal vesicles with respect to associated Rabs, PIs and intralumenal $\mathrm{pH}$. Image retrieved and modified from [235].

\subsubsection{Cargo-sorting in MVBs with respect to $H E V$}

A specific form of LEs is represented by MVBs. These vesicular structures are characterised by accumulated ILVs and are major regulators of cargo-sorting within the endosomal system. As LEs, MVBs comprise each of the afore-mentioned keyfeatures of these organelles. Further, they are defined by containing a subset of distinct membrane and intralumenal proteins [236]. The ESCRT-machinery carries a majority of these distinct proteins found on MVBs. It consists of four building blocks, which are called ESCRT0-III and mediates cargo sorting and subsequent sequestration into ILVs [237]. While manifold accessory proteins are described for all of the ESCRT subunits, only some are required to maintain its functionality. For instance, the cytosolic subunits of ESCRT-0 are majorly involved in gathering and delivering ubiquitinated cargo to ESCRT-I, thus making it non-essential for further functions of the ESCRTmachinery [238]. The latter however, is tethered to the outer surface of MVBs via so 
called FYVE zinc finger domain (FYVE-domain)-mediated interactions of accessory proteins with PI3P. Cargo-interaction and subsequent membrane-localisation is mediated via interactions with ubiquitin and respective GRAM-Like Ubiquitinbinding in EAP45 (GLUE)-domains in ESCRT subunits [239-241]. Once cargo has been transferred to the Vps23, -27 and -28-containing ESCRT-I complex, being responsible for cargo-recognition [242,243], the ESCRT-II complex is recruited. The herein included subunits Vps22, -25 and -36 condense the gathered cargo in a smaller lipid domain [244] and recruit the ESCRT-III complex. Finally, the Vps2, -20 and -24-containing ESCRT-III subunit is recruited, which de-ubiquitinates cargo and induces inwardbudding of ILVs through ATP- and Vps4-dependent polymerisation of the complex [245-247]. This ESCRT-III-spiralling and subsequent invagination of membranes is assisted by the vacuolar-sorting protein SNF7 [248]. Once membrane scission and ILVrelease occurred, the ESCRT-III complex dissociates and cargo is successfully contained within MVBs (Figure 15).

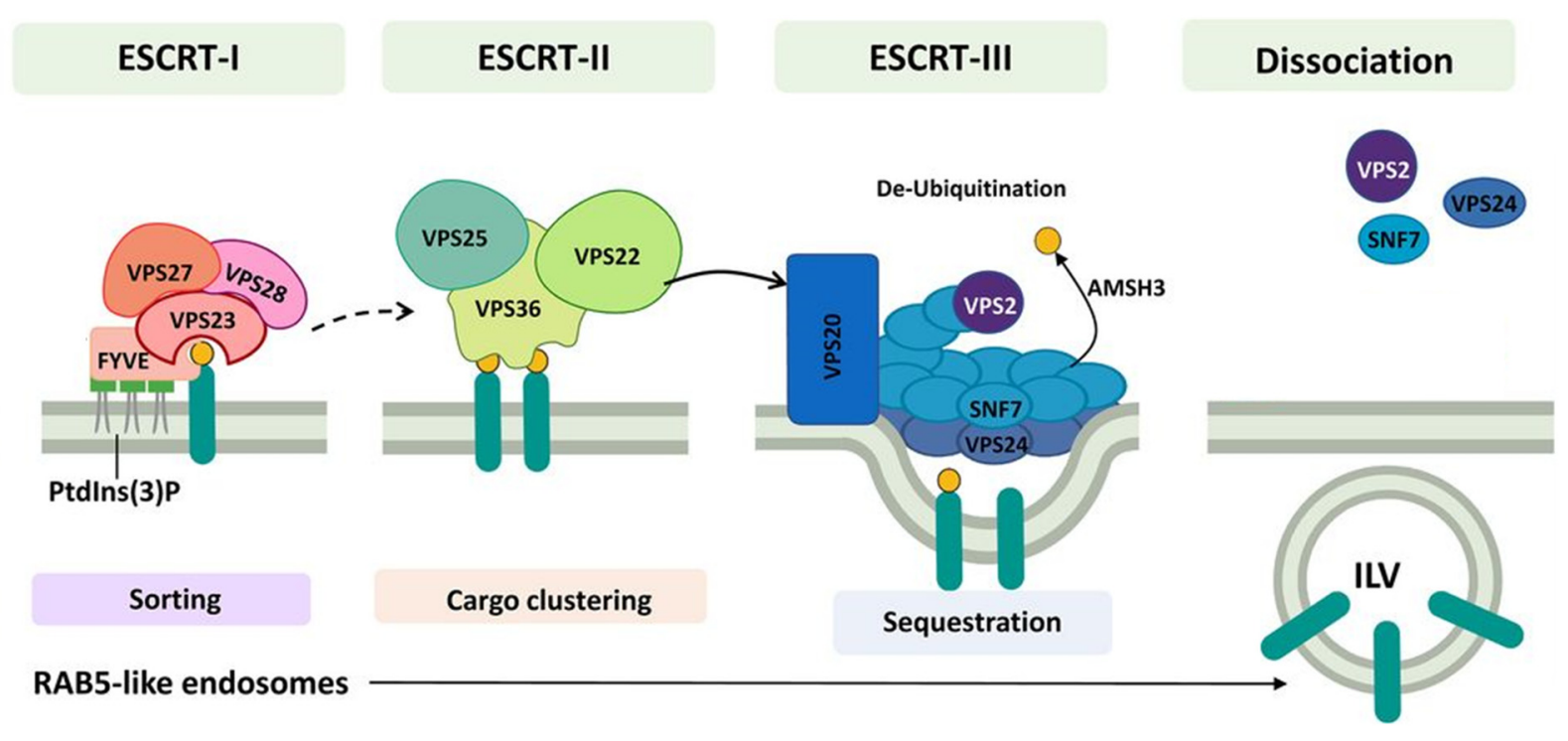

Figure 15. Ubiquitin-dependent cargo-sorting and ILV budding on MVBs via the ESCRT-machinery. Ubiquitinated cargo is recognised and bound by ESCRT-I on the surface of MVBs. Subsequent recruitment of ESCRT-II leads to clustering of cargo within lipid microdomains, thereby concentrating ubiquitinated proteins. Finally, ESCRT-III is recruited which leads to inward-budding of the MVB membrane through polymerisation, thereby creating ILV-precursors. After membrane-scission, ILVs are released into MVBs and the ESCRT-complex dissociates to start a new cycle. Image retrieved and modified from [249].

Apart from ubiquitin-based cargo-sorting, molecules can be shuttled to the ESCRTmachinery via various different mechanisms. This cargo can represent proteins, which were described to be delivered to the ESCRT-complexes by e.g. Alix [250], hepatocyte growth factor-regulated tyrosine kinase substrate (Hrs) [251] or tetraspanins [252]. As alternate cargo-receptors, they subsequently make use of the already MVB-localised sorting complexes. Similarly, non-protein cargo such as lipids or RNA can be transferred into ILVs [253-255]. 
One rather interesting aspect and alternate route of cargo-sorting in MVBs is the use of TSG101 (Vps23)-interactions within the ESCRT-I-complex. First described for the gag-protein of HIV, viruses can induce an interaction with the sorting complex via so called viral late domains [256,257]. For HEV, such a late domain was characterised as part of pORF3, which carries a classical PxxP-motif in its C-terminal portion. For all described HEV genotypes, this is specifically formed by the amino acid sequence PSAP [34], which directly interacts with TSG101 [103,105]. As MVBs may fuse with the PM, ILVs are subsequently released as so called exosomes, depending on associated cargosorting factors [258,259]. Similarly, ILV-incorporated HEV capsids containing prenylated pORF3 are released as quasi-enveloped or exosomal particles in a Rab27adependent manner [260]. As such, this lipid envelope of eHEV virions carries several proteins conventionally found within or associated with MVBs, suggesting that the exosomal pathway represents the major viral release mechanism [100,106]. These cellular factors are represented by e.g. the tetraspanins cluster of differentiation 9 (CD9), CD63 or CD81, parts of the ESCRT-machinery such as Alix, Hrs, TSG101 or Vps4 and finally the TGN-protein trans-Golgi network integral membrane protein 2 (TGOLN2). This quasi-envelope is retained within the blood-stream, possibly rendering a broad tissue-specificity and immune-evasion of eHEV [261,262], and is lost once virions enter the biliary duct (Figure 16). 


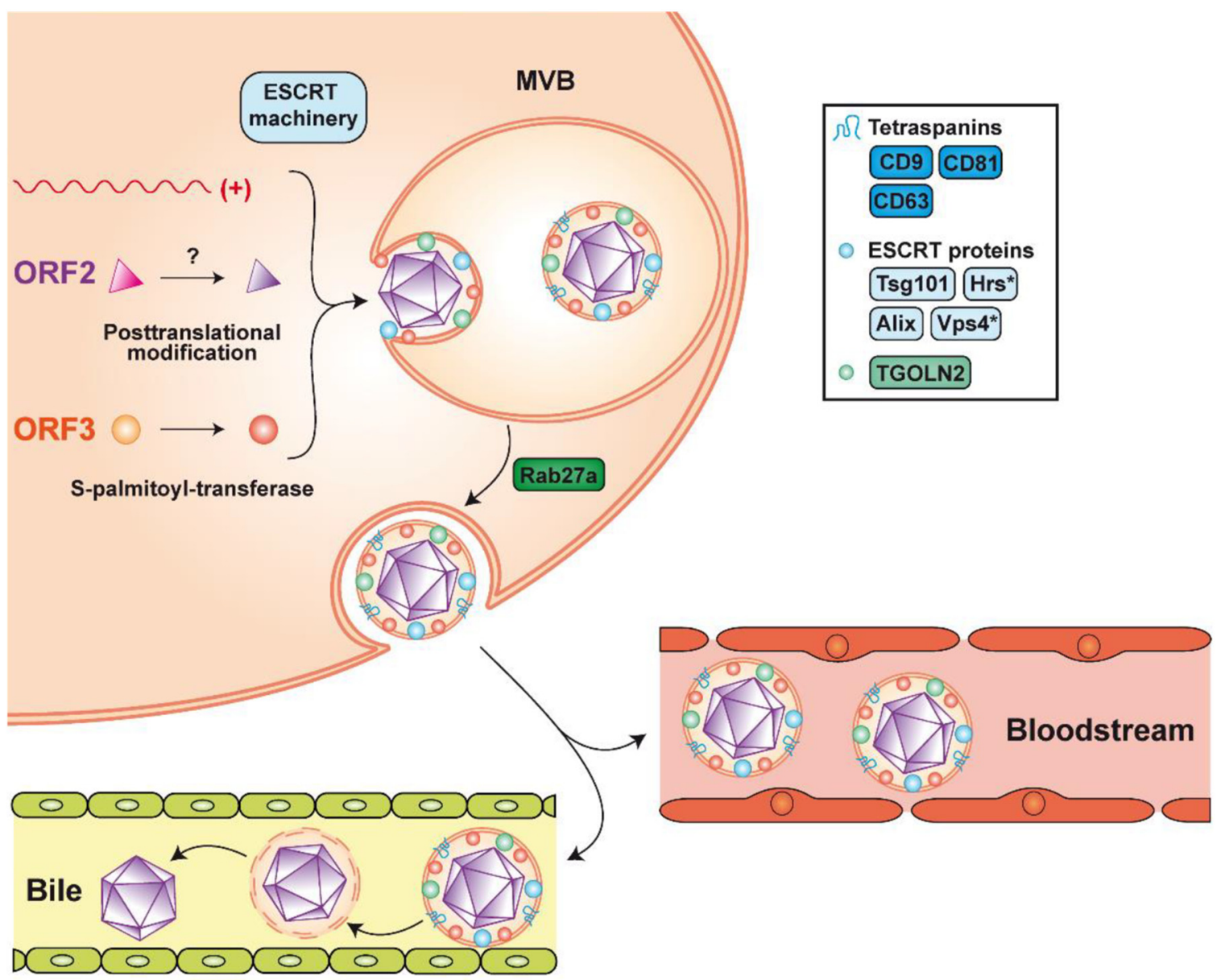

Figure 16. Endosomal incorporation of HEV virions. Upon morphogenesis of the pORF2- and genomic RNAcontaining HEV capsids, palmitoylated pORF3 interacts with the structure. This leads to TSG101- and ESCRTdependent incorporation into ILVs of MVBs. These ILVs carry cellular factors such as CD9, -63, -81, TSG101, Hrs, ALIX, Vps4 or TGOLN2. Subsequently, HEV-containing MVBs shuttle towards the PM in a Rab27a-dependent manner, where they fuse and release intralumenal cargo into the extracellular space. Thereby, HEV virions retain their ILV-originating envelope along with cellular host-factors while circulating in the blood. Upon exposure to bile-salts in the biliary duct, the quasi-envelope is removed and HEV is excreted as naked capsid virus. Image retrieved from [263].

\subsubsection{Cholesterol-dependent regulations in the endolysosomal system}

As the endolysosomal system requires continuous membrane-fission, -fusion and curvature events to maintain an overall vesicular flux, it is highly dependent on lipid composition of vesicular membranes. Herein, an abundant and central key-player is represented by cholesterol and its derivatives, affecting all of the above mentioned mechanisms [264-266]. Generally, the abundance of cholesterol within a lipid bilayer has various impacts on its properties, such as membrane thickness or fluidity $[267,268]$. This in turn alters proteins being clustered in certain domains of the bilayer with respect to e.g. the length of their transmembrane domain (TMD) [269], thereby also affecting lipid raft-like structures as functional domains in the endolysosomal system [270-272]. Additionally, the presence of cholesterol facilitates membrane curvature to some extent [273]. This proves important for endosomal ILV-formation, especially for 
the ESCRT-machinery. Here, functional domain clustering, as well as cargo-sorting and ILV-scission depend on and are partially regulated by the lipid [244,274-276].

Apart from intralumenal processes in late endosomes, cholesterol further is important in organising membrane fusion of vesicles flowing into the endolysosomal system. These processes are mainly mediated by SNARE-proteins, which in turn are trafficked to the place of action $[277,278]$, sorted in place $[279,280]$ and controlled with respect to successful membrane fusion [281] in a cholesterol-dependent manner. Just as SNARE-trafficking, retrograde vesicular trafficking and build-up of ER-vesicle contact-sites is orchestrated by cholesterol. This is achieved by cholesterol-binding proteins being responsible for recruiting different sets of accessory proteins to vesicular structures. Master regulators of these processes are the oxysterol-binding protein-related protein 1 (ORP1L) and Rab-interacting lysosomal protein (RILP) [282284]. Together they mediate LE translocation by selectively regulating association with the motor complex Dynein/Dynactin, thereby directing retrograde trafficking of LEs towards lysosomes along MTs [285,286]. Besides cholesterol directly mediating interactions of Rab-effectors such as SNAREs, RILP or ORP1L, it also plays a crucial role in controlling Rab-prenylation. With changing cholesterol-levels, cholesterol biosynthesis is impacted. High cellular cholesterol herein leads to a drop in 3-hydroxy3-methyl-glutaryl-coenzyme A reductase (HMGCR)-expression [287-289]. As HMGCR represents the rate-limiting step in cholesterol-biosynthesis [290], a drop in productivity also renders further catalytic steps, crucial for providing substrates for protein prenylation, to be less efficient. A lack in proper protein-prenylation also decreases efficient prenylation-dependent shuttling of Rab-proteins to respective endosomes [291], leading to a lack in effector-recruitment and to an overall impairment of endocytic flux and endosome-motility [292,293].

The above described mechanisms of cholesterol controlling the maturation, processivity and trafficking of vesicles within the endosomal system can be summarised as the lipid directly affecting the following aspects: (i) organisation of proteins in functional microdomains, (ii) facilitation of membrane curvature, (iii) induction of ILV-scission, (iv) regulation of membrane-fusion events, (v) control of vesicle-transport directions, (vi) regulation of Rab-functionality and recruitment of Rab-effector molecules to their destination (Figure 17). 


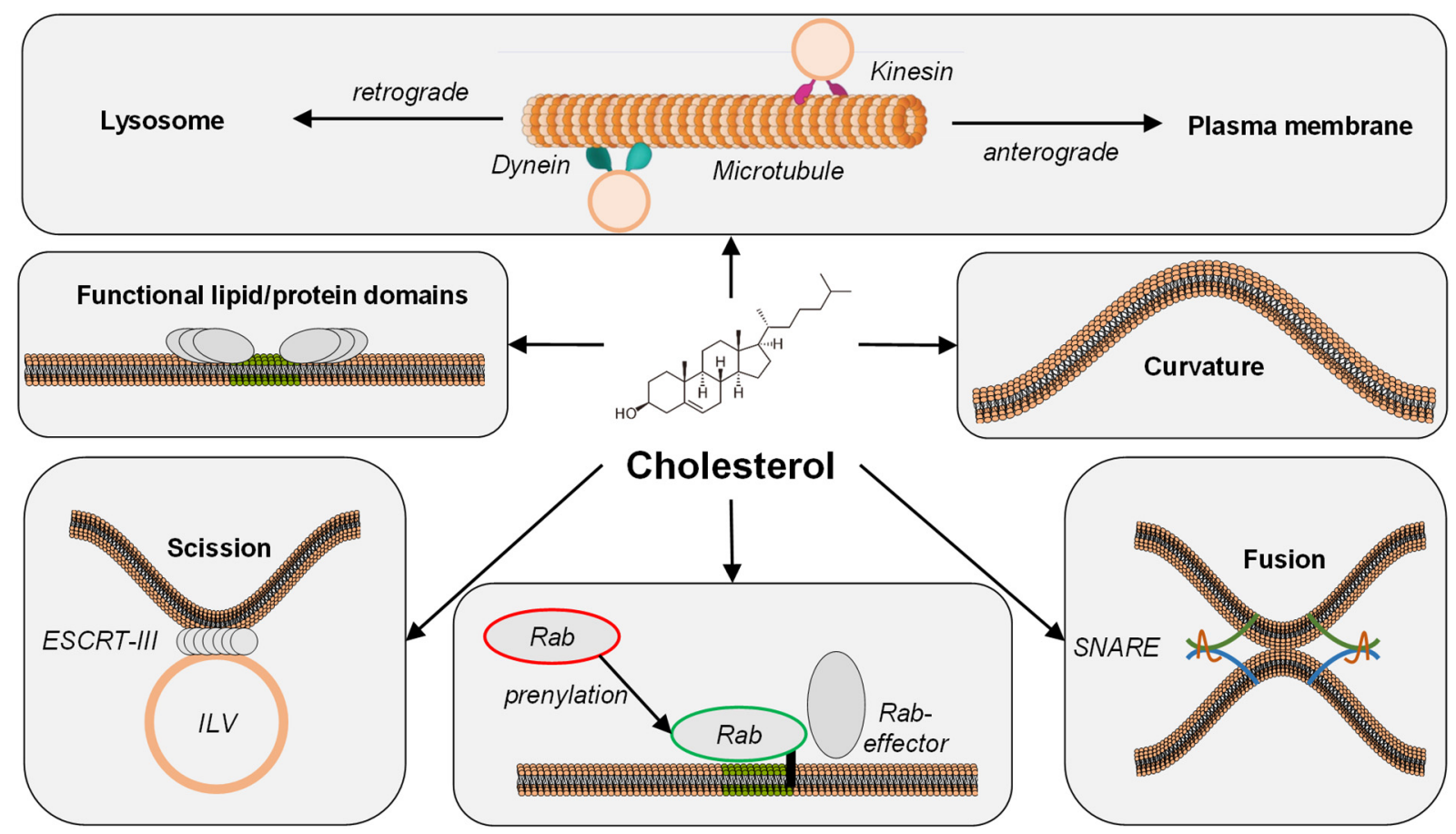

Figure 17. Regulatory processes of cholesterol in the endolysosomal system. Cholesterol directly is involved in building up lipid microdomains containing distinct proteins of different functions. These, together with cholesterol exerting bending effects on the membrane, facilitate induction of membrane-curvature. Protein- or cholesterolassisted bent membranes are crucial for both fission and fusion events. ESCRT-III-induced membrane-scission is the fundament of ILV-formation within LEs, whereas SNARE-mediated membrane-fusion is crucial for maintaining endosomal flux, both of which are, in parts, controlled by cholesterol. In this context, recruitment of accessory proteins exerting effects on the membrane and orchestrating endosome-trafficking, is mediated by prenylated Rab, which in turn is dependent on an intact cholesterol synthesis. This makes cholesterol a central regulator in the endolysosomal system.

\subsection{Cellular cholesterol homeostasis and pharmacological modulation}

Within a cell, the amount and localisation of cholesterol is strictly regulated to maintain cellular processes and to prevent cytotoxic effects. The lipid can either be taken up exogenously via lipoproteins, most prominently via low-density lipoprotein (LDL), or can be synthesised within a cell. Processes involved in either of these regulate one another via transcription or protein degradation, thereby using a complex system depending on manifold cellular factors.

LDL particles are members of the lipoprotein particle family, which are responsible for carrying lipids through an organism. Apart from LDL they include chylomicrons, very low-density lipoproteins (VLDL), intermediate-density lipoproteins (IDL) and highdensity lipoproteins (HDL). Each consists of a lipophilic core with distinct lipidcomposition, which is surrounded by a phospholipid-membrane and accessory proteins $[294,295]$. These complexing proteins also determine targeting of cells, as they interact with specific receptor. In case of LDL, which mostly carries cholesterol, the surface protein apolipoprotein B100 (ApoB100) interacts with the LDL-receptor (LDLR) on e.g. hepatocytes to induce receptor-mediated endocytosis [296]. From this 
point on, LDLR-bound LDL particles are carried towards EEs, where the complex dissociates and LDLR may be recycled via REs [297,298] or targeted for degradation via interaction with proprotein convertase subtilisin/kexin type 9 (PCSK9) [299]. LDL is then further shuttled to LEs and lysosomes, where it is degraded via lysosomal acid lipase (LAL) and cholesterol is freed from the particle [300]. Once this is achieved, cholesterol is shuttled from the lysosome to other organelles with the help of lysosomal cholesterol-binding protein such as lysosome-associated membrane glycoprotein 2 (LAMP2) [301], Saposins (Saps) [302], lysosomal integral membrane protein-2 (LIMP2) [303] or the Niemann-Pick disease, type $C 1$ intracellular cholesterol transporter 1 (NPC1) and NPC2 system [304,305]. The transfer of cholesterol from lysosomes to respective target organelles is hereby dependent on recruited contact-mediators such as Synaptotagmin-7 (Syt7) for peroxisomes [306], Mitofusin 2 (Mfn2) and Vps13A for mitochondria [307,308] or NPC1, ORP1L and RILP for the ER [284,309]. Once cholesterol reached the ER, it is esterified with fatty acids in an Acyl-CoA-cholesterolAcyltransferase (ACAT)-dependent manner and subsequently stored in lipid droplets (LDs) [310]. This essentially abrogates biological activity of cholesterol and withdraws it from the cycle (Figure 18).

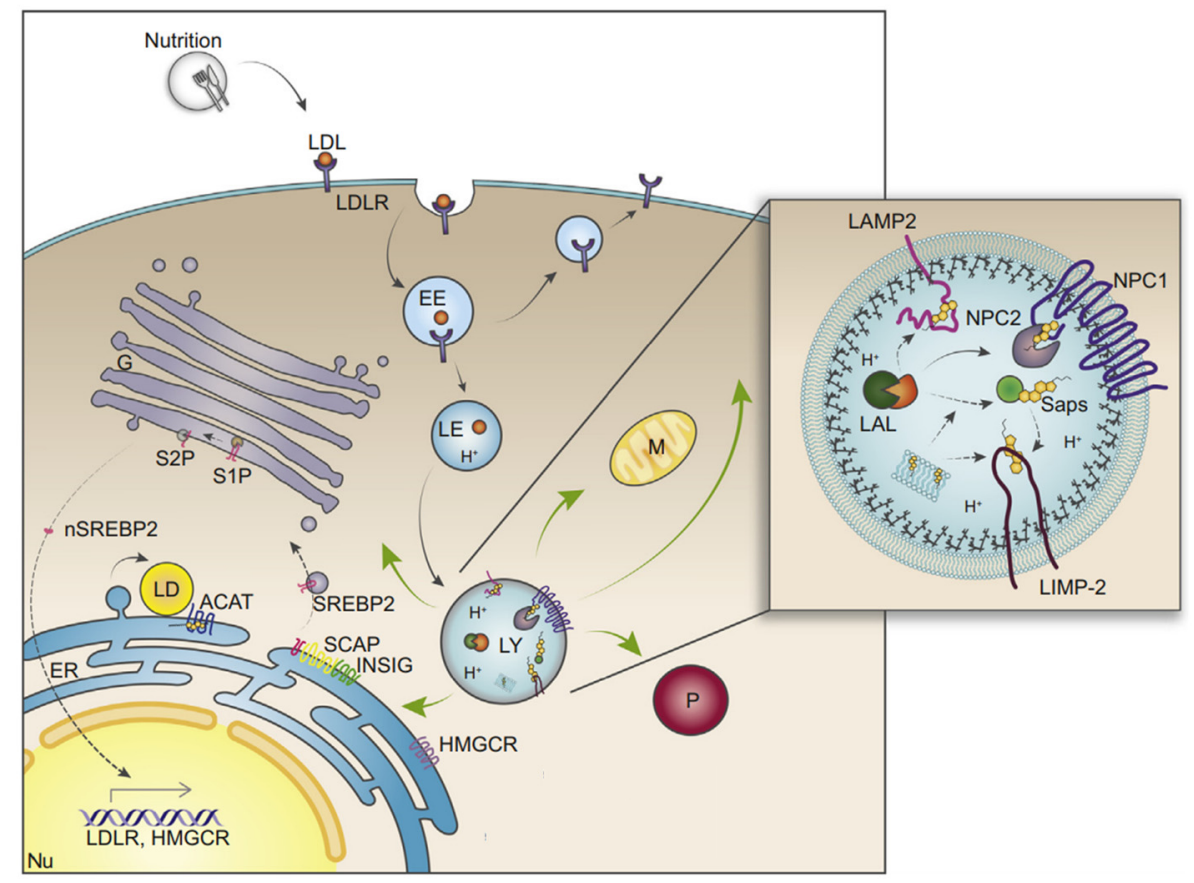

Figure 18. LDL-uptake and its resulting transcriptional changes. LDL enters the cell via receptor-mediated endocytosis. Once inside EEs, LDLR can either be recycled to the PM or further transferred to LEs in a PCSK9dependent manner. Via LEs, receptor-dissociated LDL is shuttled into lysosomes (LY). There, cholesterol is freed from LDL particles via LAL and passed on to different organelles with the help of LAMP2, LIMP-2, NPC1/2 or Saps. Major organelles being targets of these re-distributions are mitochondria (M), peroxisomes (P) or the ER. Within the ER, cholesterol is esterified with fatty acids via ACAT. Further, it stabilises the SREBP-SCAP-INSIG complex in the ER-membrane, preventing SREBP-shuttling to the Golgi and subsequent S1P/S2P-mediated activation of the transcription factor. This leads to repression of SREBP-dependent expression of both HMGCR and LDLR, inhibiting both LDL-uptake and cholesterol-biosynthesis. Image retrieved and modified from [311]. 
Apart from being stored via the ER, cholesterol here also fulfils regulatory functions with respect to transcription. Abundance of the lipid stabilises a complex containing the transcription factors sterol regulatory element-binding proteins (SREBPs) and the associated proteins SREBP cleavage activating protein (SCAP) and Insulin induced gene 1 (INSIG1) [312]. While interacting, this complex remains in the ER membrane without fulfilling transcriptional activation of sterol-regulatory element (SRE)containing genes. However, once cholesterol-concentrations within the ER drop, INSIG1 dissociates and the SCAP-SREBP-complex is shuttled to the Golgi-apparatus [313]. There, SREBPs are cleaved by site-1- and site-2-proteases (S1P/S2P) [314,315], releasing the transcription factor domain from the membrane [316], which subsequently is shuttled to the nucleus via Importin. Finally, expression of genes being involved in cholesterol-uptake and -synthesis such as LDLR or HMGCR [317] are induced (Figure 18), consequently elevating intracellular cholesterol levels [318].

Similar to this regulatory system, cholesterol levels are affected by other transcription factors such as liver $X$ receptors (LXRs), farnesoid $X$ receptors (FXRs), retinoid $X$ receptors (RXRs) or peroxisome proliferation-activated receptors (PPARs), all of which are agonised or antagonised by cholesterol and its derivatives such as oxysterols [319323]. Induction and repression of gene-expression exerted by these transcription factors results in a complex system of cholesterol re-distribution and removal. A direct way in achieving this is using cholesterol-exporters and adaptor proteins delivering the substrate. To a large extent, these export-processes and related factors are transcriptionally controlled via the LXR-isoform LXR $\alpha$ rather than LXR $\beta$ [324]. These transcription factors form heterodimers with RXRs or PPARs, which is crucial for subsequent effects on gene-expression [325-327]. Concordantly, induction of LXRactivity is mediated by either its own agonists, cholesterol and oxysterols [328-330], or the agonists of its partner within the heterodimer. Alongside minor effectors of direct cholesterol-catabolism, some ATP-binding cassette transporters (ABC-transporters) are induced, which specifically bind cholesterol and oxysterols and transport the molecules into the extracellular space. One example is represented by ABC-transporter A1 (ABCA1), which aids in removal of cholesterol from lysosomes via the NPC1/2 system [331]. The latter transfers cholesterol to the first, which passes the lipid on to apolipoprotein A-I (apoA-I) [332,333]. Ultimately, this leads to removal of intracellular cholesterol via apoA-I-dependent formation of HDL with the help of ABC-transporter G1 (ABCG1) [334,335]. LXR-activity further is modulated by several co-activators or repressors. One of these is represented by the transcription factor nuclear factor erythroid 2-related factor 1 (Nrf1), which is a dual sensor for both cholesterol and reactive oxygen species (ROS) [336-338]. Similar to SREBPs, the absence of cholesterol leads to a proteolysis- and translocation-based activation of Nrf1 and a subsequent repression of LXR. On the contrary, abundance of cholesterol results in retention of 
Nrf1 within the ER, inactivating the transcription factor [339,340]. This lack of effect further leads to induction of inflammation [338], which can be correlated with a cholesterol-dependent activation of the inflammasome [341] in the context of a PRRactivation [342], thereby linking cholesterol- and PAMP-based transcriptional processes.

Besides cholesterol- and oxysterol-removal via exporters, cholesterol levels can also be lowered via derivatisation into bile acids. A transcriptional key-regulator of enzymes involved in bile acid formation is FXR. Similar to LXRs, it forms heterodimers with RXRs [343] to induce or repress gene-expression in this context [344]. Its natural agonist is farnesol [345], which proves as important substrate in cholesterolbiosynthesis in form of farnesyl pyrophosphate (FPP), thereby rendering regulation of FXR by cholesterol anabolism [346] with associated cholesterol-clearance. Apart from farnesol, primary bile acids such as chenodeoxycholic acid control FXR-activity, thereby regulating bile acid synthesis [347-349]. FXR is further co-regulated by PPARs, where PPAR $\alpha$ exerts inhibitory functions towards FXR [350,351]. Generally, bile acid synthesis is started by LXR-induced cholesterol-oxidation via either the ER-resident cholesterol 7 alpha-hydroxylase (CYP7A1) or the mitochondrial sterol 27-hydroxylase (CYP27A1) [352,353], both of which represent committed steps in the classical and the acidic alternate bile acid synthesis, respectively [354-358]. Three major organelles are involved in bile acid synthesis: (i) the ER, (ii) mitochondria and (iii) peroxisomes [359361]. Substrate distribution is hereby achieved through cholesterol-transport mechanisms explained above and mediated through organelle-organelle contact sites. Once bile acid synthesis is completed, a negative feedback loop is in place, rendering FXR to inhibit primary bile acid synthesis [362]. Further, the amphiphilic molecules are shuttled out of the cell via a variety of different transporters or conjugated to amino acids via e.g. bile acid-CoA amino acid N-acyltransferase (BAAT) [363], which are then exported. While the classical exporter for bile acids was described as bile salt export pump (BSEP/ABCB11) [364-367], alternative exporters are represented by either canalicular multispecific organic anion transporter 2 (MRP2/ABCC2) [367] or organic solute transporter subunit alpha (OST $\alpha)$ and OST $\beta$ [368]. While the first is majorly transcriptionally induced by FXR [369], expression of the latter are induced by both FXR and LXR [370-372], again proving the strong interaction between the different cholesterol-modulated transcription factors. In principal, the synthesis of bile acids and amino acid-conjugated bile acids and direct cholesterol-export represent the most efficient ways to rid a cell from cholesterol.

Together with the cholesterol-anabolic regulators SREBP, the majorly catabolic regulators FXR, LXR, PPAR and RXR form a cycle of maintaining cholesterolhomeostasis under mutual induction. This is achieved by build-up and removal of 
respective substrates acting as agonists or antagonists of associated transcription factors [373-375] (Figure 19).

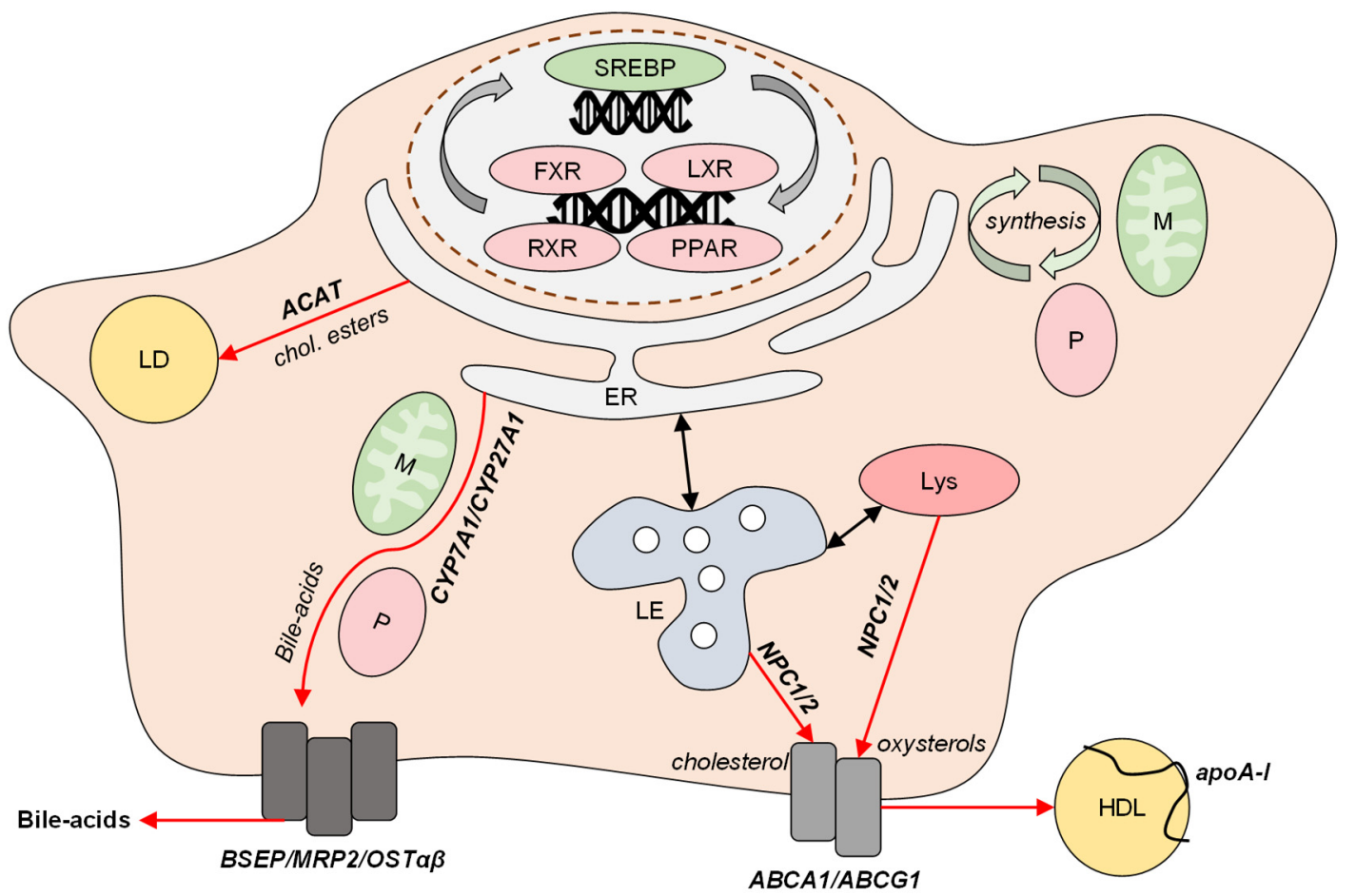

Figure 19. Cholesterol-removal processes in a human hepatocyte. Cholesterol biosynthesis is regulated by SREBPS and is catalysed within the ER, peroxisomes $(\mathrm{P})$ and mitochondria $(\mathrm{M})$. Upon cellular re-distribution and rising cholesterol-levels, LXR-, FXR-, PPAR- and RXR-mediated cholesterol-removal processes are induced. Inactivation of cholesterol is achieved via esterification with fatty acids in an ACAT-dependent way with cholesteryl-esters being stored in lipid droplets (LD). A further way of decreasing intracellular cholesterol is direct export of the lipid or oxysterols via late endosomes (LE) or lysosomes (Lys). These transfer cholesterol e.g. via NPC1/2 to the transporters ABCA1 and ABCG1, which aid in building up apoA-I-complexed high-density lipoprotein (HDL). A third cholesterol-removal program is induced upon bile acid synthesis, which is engaged by either CYP7A1 or CYP27A1 as they catalyse the committed steps of classical and acidic alternate synthesis of the derivatives. Bile acids synthesised via catalytic processes in ER, $\mathrm{M}$ or $\mathrm{P}$, are then exported from cells via the transporters BSEP, MRP2 or OST $\alpha / \beta$. In principle, build-up of cholesterol and its primary derivatives induces its removal pathways. Opposing to this, ridding a cell of cholesterol induces its synthesis. Through this mechanism, related transcription factors regulate each other via supplying agonists and antagonists. Removal routes of cholesterol are marked with red arrows, whereas contributing transcription factors are coloured red. Synthesis of cholesterol is marked with green arrows, whereas contributing transcription factors are coloured green.

From a clinical point of view, the strict regulation of cholesterol-metabolism and homeostasis can be exploited to achieve certain phenotypes. In the majority of cases, an overall aim is the reduction of cholesterol in patients suffering from hypercholesterolemia, atherosclerosis or cholestasis. Here, a variety of different lipid lowering drugs are available on the market or are subject to clinical trials and act through different mechanisms.

In the late 1950's, first steps towards the discovery of cholesterol- and lipid-lowering drugs was achieved by establishing the use of Fibrates [376]. Following application of Clofibrate, a variety of different derivatives were discovered and released on the market, including compounds such as Bezafibrate, Fenofibrate or Gemfibrozil 
[377,378]. These drugs act as agonists of PPAR $\alpha$, which effectively leads to a suppression of bile acid synthesis. A consequence is a retention of cholesterol within the cell and therefore triggers a reduced serum-cholesterol concentration and reduction in biliary sterols [379,380]. About a decade later, in the early 1970's [381], another family of lipid-lowering drugs was discovered: Statins. These were more selective towards cholesterol and ever since represented the most frequently used drug to treat hypercholesterolemia. Included in this drug family are compounds such as Pravastatin, Fluvastatin, Atorvastatin or Simvastatin. Mechanistically, these compounds act through inhibition of HMGCR, thereby inhibiting cholesterol biosynthesis [381,382]. A result is a decrease in intracellular cholesterol, hence a reduction in systematic cholesterol [383]. Apart from Fibrates and statins, a third group of small-molecule cholesterol-modulators was under discussion for being used in clinic. Here, compounds such as Lecimibid or Avasimibe were used to inhibit ACAT, therefore interfering with cholesterol-storage in LDs, which subsequently can be detoxified more efficiently [384,385]. Despite there being promising studies, ACATinhibitors were never considered for cholesterol-targeted use in clinics.

More novel approaches of lowering systemic cholesterol make use of larger molecules such as cyclosporin A-derivatives, hormones or antibodies. Initially developed as a compound aiding in chemotherapy, the cyclosporin A-derivative PSC833 inhibits pglycoproteins. Its primary targets are $\mathrm{ABC}$-transporters, which results in a reduction in multiple drug resistance [386]. However, by inhibiting the ABC-transporter ABCA1, PSC833 also poses inhibitory functions for cholesterol-efflux from cells [387]. Consequently, cellular cholesterol levels rise upon application. However, clinical approval of the compound is not yet completed. Another candidate to be approved for clinical use is the FGF19-analogue NGM282. Acting as a hormone, this analogue inhibits CYP7A1-expression via FXR and therefore bile acid synthesis [388,389]. As a consequence, cholesterol is not detoxified via bile acids, but via HDL-formation [390]. Lastly, a new generation of systemic cholesterol-lowering drugs is represented by the monoclonal antibodies Evolocumab and Alirocumab. These specifically bind PCSK9 on the cell-surface and lead to its inactivation [391]. In essence, this prevents LDLRdegradation and increases its amount on the cell surface through enhanced receptorrecycling, thereby increasing LDL-uptake and its clearance from serum [392,393].

Taken together, all of the above mentioned compounds achieve the overall aim to reduce serum-cholesterol. However, statins differ from the others with respect to what this means for intracellular cholesterol levels. While statins generally reduce cholesterol-biosynthesis, therefore reducing cellular cholesterol, compounds such as Fibrates, Avasimibe, PSC833, FGF19 and Alirocumab lead to increased cholesteroluptake or retention of the lipid within the cell. This in turn will be of central interest for this study and is to be highlighted. 


\section{Aim of this study}

The Hepatitis E virus, the major agent inducing an acute viral hepatitis worldwide, is a grossly understudied virus rendering both cellular defence-mechanisms and the viral life cycle remaining elusive in large parts. As a consequence, antiviral strategies are sparse, unspecific and associated with severe adverse effects. Understanding how HEV modulates the innate immune-response, which effector molecules are induced by and efficient against the virus and how this can be used in an antiviral strategy therefore represents the overall aim of this study.

In context of innate immunity, elucidation of antiviral mechanisms exerted by one of the most prominently induced ISGs, GBP1, was set to be clarified in more detail. How HEV modulates this host-factor and what consequences on the viral life cycle are triggered by an abundance of it shall be elucidated. Further, different modes of action of GBP1 are to be analysed with regards to the protein's dynamin-function, GTPaseactivity and involvement in endolysosomal processes. As HEV is released via exosomes generated within MVBs, the latter fulfil a pivotal role in the viral life cycle. These structures are highly dependent on cellular cholesterol homeostasis. Thus, effects exerted by an HEV-infection on cellular cholesterol, as well as effects exerted by the lipid on the virus shall be studied. A focus herein is set on how cholesterol affects the fate of progeny viruses under use of pharmacological modulation. Ultimately, the question to be answered is whether targeting the cellular cholesterol content may serve as antiviral target against HEV.

In essence, this study will help to understand underlying effects of an already used antiviral, namely IFN, and how this is connected to cholesterol and degradative processes within an infected cell. This will help improving basic understanding of the viral life cycle and will lead to discovery of a more precisely targeted therapy. 
3. Materials

3.1. Buffers and solutions

\begin{tabular}{|c|c|}
\hline Buffer or solution & Ingredients \\
\hline \multirow[t]{2}{*}{ Anode buffer I } & $20 \%(v / v) \mathrm{EtOH}$ \\
\hline & $0.3 \mathrm{M}$ Tris \\
\hline \multirow[t]{2}{*}{ Anode buffer II } & $20 \%(v / v) \mathrm{EtOH}$ \\
\hline & $25 \mathrm{mM}$ Tris \\
\hline \multirow[t]{4}{*}{ Carbazole stain solution } & $30 \mathrm{mM} \mathrm{NaOAc}$ \\
\hline & 12 mM AcOH (glacial) \\
\hline & $0.05 \%(\mathrm{w} / \mathrm{v})$ 3-amino-9-ethylcarbazole \\
\hline & $0.01 \%(\mathrm{v} / \mathrm{v}) \mathrm{H}_{2} \mathrm{O}_{2}$ \\
\hline \multirow[t]{2}{*}{ Cathode buffer } & $20 \%(v / v) \mathrm{EtOH}$ \\
\hline & $40 \mathrm{mM}$ 6-aminohexanoic acid \\
\hline \multirow[t]{7}{*}{ Firefly luciferase substrate } & 20 mM Tris- $\mathrm{HCl}$ \\
\hline & $5 \mathrm{mM} \mathrm{MgCl} 2$ \\
\hline & $0.1 \mathrm{mM}$ EDTA \\
\hline & $33.3 \mathrm{mM}$ DTT \\
\hline & $470 \mu \mathrm{M}$ D-luciferin \\
\hline & $530 \mu \mathrm{M}$ ATP \\
\hline & $\mathrm{pH} 7.8$ \\
\hline \multirow[t]{6}{*}{ Luciferase lysis buffer } & $25 \mathrm{mM}$ Tris- $\mathrm{HCl}$ \\
\hline & $2 \mathrm{mM}$ DTT \\
\hline & 2 mM EGTA \\
\hline & $10 \%(v / v)$ glycerol \\
\hline & $0.1 \%(\mathrm{v} / \mathrm{v})$ Triton $\mathrm{X}-100$ \\
\hline & $\mathrm{pH} 7.5$ \\
\hline \multirow[t]{5}{*}{ Mowiol mounting medium } & 100 mM Tris/HCl \\
\hline & $10 \%(w / v)$ Mowiol \\
\hline & $25 \%(\mathrm{w} / \mathrm{v})$ glycerol \\
\hline & $2.5 \% \mathrm{DABCO}$ \\
\hline & $\mathrm{pH} 8.5$ \\
\hline PBS (Phosphate Buffered Saline) & $137 \mathrm{mM} \mathrm{NaCl}$ \\
\hline \multirow[t]{3}{*}{ without $\mathrm{Mg}^{2+}$ and $\mathrm{Ca}^{2+}$} & $2.7 \mathrm{mM} \mathrm{KCl}$ \\
\hline & $8.1 \mathrm{mM} \mathrm{Na}_{2} \mathrm{HPO}_{4}$ \\
\hline & $\mathrm{pH} 7.4$ \\
\hline \multirow[t]{6}{*}{ RIPA lysis buffer } & 50 mM Tris/HCl \\
\hline & $150 \mathrm{mM} \mathrm{NaCl}$ \\
\hline & $0.1 \%(\mathrm{w} / \mathrm{v}) \mathrm{SDS}$ \\
\hline & $1 \%(\mathrm{w} / \mathrm{v})$ sodium deoxycholate \\
\hline & $1 \%(v / v)$ Triton X-100 \\
\hline & $\mathrm{pH} 7.2$ \\
\hline
\end{tabular}




\begin{tabular}{ll}
\hline SDS-loading buffer (4x) & $4 \%(\mathrm{w} / \mathrm{v}) \mathrm{SDS}$ \\
& $125 \mathrm{mM}$ Tris/HCl \\
& $10 \%(\mathrm{v} / \mathrm{v})$ glycerol \\
& $10 \%(\mathrm{v} / \mathrm{v}) \beta$-mercaptoethanol \\
& $0.02 \%(\mathrm{w} / \mathrm{v})$ bromophenol blue \\
& $\mathrm{pH} 6.8$ \\
\hline SDS-running buffer (10x) & $0.25 \mathrm{M}$ Tris $\mathrm{pH} 8.3$ \\
& $2 \mathrm{M}$ glycine \\
& $1 \%(\mathrm{w} / \mathrm{v}) \mathrm{SDS}$ \\
\hline SDS-separating gel buffer & $1.5 \mathrm{M}$ Tris \\
& $0.4 \%(\mathrm{w} / \mathrm{v}) \mathrm{SDS}$ \\
& $\mathrm{pH} 8.8$ \\
\hline SDS-stacking gel buffer & $0.5 \mathrm{M} \mathrm{Tris}$ \\
& $0.4 \%(\mathrm{w} / \mathrm{v}) \mathrm{SDS}$ \\
& $\mathrm{pH} 6.7$ \\
\hline TBS (1x) & $20 \mathrm{mM}$ Tris \\
& $150 \mathrm{mM} \mathrm{NaCl}$ \\
& $\mathrm{pH} 8.8$ \\
\hline TBS-T (1x) & $20 \mathrm{mM}$ Tris \\
& $150 \mathrm{mM} \mathrm{NaCl}$ \\
& $0.05 \%(\mathrm{v} / \mathrm{v})$ Tween-20 \\
& $\mathrm{pH} 8.8$ \\
\hline & $0.05 \%(\mathrm{w} / \mathrm{v})$ trypsin \\
& $0.02 \%(\mathrm{w} / \mathrm{v})$ EDTA \\
& in PBS \\
\hline
\end{tabular}

3.2. Growth media

\begin{tabular}{lll}
\hline Medium & Ingredients & Supplier \\
\hline $\begin{array}{l}\text { DMEM (Dulbecco's } \\
\text { Modified Eagle's } \\
\text { Medium) High Glucose }\end{array}$ & Biowest, Nuaillé, France \\
& & \\
\hline DMEM complete & $\begin{array}{l}\text { DMEM High Glucose } \\
10 \%(\mathrm{v} / \mathrm{v}) \mathrm{FCS}\end{array}$ & \\
& $2 \mathrm{mM}$ L-glutamine \\
& $100 \mu \mathrm{g} / \mathrm{mL}$ streptomycin & \\
& $100 \mathrm{U} / \mathrm{mL}$ penicillin & \\
\hline $\begin{array}{l}\text { Foetal bovine serum } \\
\text { (FBS/FCS) }\end{array}$ & & $\begin{array}{l}\text { Bio \& Sell GmbH, Feucht, } \\
\text { Germany }\end{array}$ \\
\hline $\begin{array}{l}\text { Opti-MEM } \\
\text { serum medium }\end{array}$ & & $\begin{array}{l}\text { Thermo-Scientific, } \\
\text { Braunschweig, Germany }\end{array}$ \\
\hline
\end{tabular}




\begin{tabular}{ll}
\hline Serum-free DMEM & DMEM High Glucose \\
& $2 \mathrm{mM}$ L-glutamine \\
& $100 \mu \mathrm{g} / \mathrm{mL}$ streptomycin \\
& $100 \mathrm{U} / \mathrm{mL}$ penicillin \\
\hline Serum-reduced DMEM & DMEM High Glucose \\
& $1 \%(\mathrm{v} / \mathrm{v})$ FCS \\
& $2 \mathrm{mM}$ L-glutamine \\
& $100 \mu \mathrm{g} / \mathrm{mL}$ streptomycin \\
& $100 \mathrm{U} / \mathrm{mL}$ penicillin \\
\hline
\end{tabular}

\subsection{Antibodies}

\subsubsection{Primary antibodies}

\begin{tabular}{|c|c|c|c|}
\hline Reactivity & Host & Cat. No. & Supplier \\
\hline $\begin{array}{l}\text { Flag-tag } \\
\text { (monoclonal) }\end{array}$ & Mouse & 66008-3-Ig & $\begin{array}{l}\text { Proteintech, } \\
\text { Rosemont, USA }\end{array}$ \\
\hline $\begin{array}{l}\text { HEV capsid } \\
\text { protein } \\
\text { (monoclonal) }\end{array}$ & Mouse & Clone 5G5 & Prof. Jihong Meng \\
\hline $\begin{array}{l}\text { HEV capsid } \\
\text { protein } \\
\text { (polyclonal) }\end{array}$ & Rabbit & HCD3K129 & Mirco Glitscher \\
\hline $\begin{array}{l}\text { human GAPDH } \\
\text { (monoclonal) }\end{array}$ & Mouse & sc-32233 & $\begin{array}{l}\text { SantaCruz } \\
\text { Biotechnology, } \\
\text { Heidelberg, } \\
\text { Germany }\end{array}$ \\
\hline $\begin{array}{l}\text { human GBP1 } \\
\text { (polyclonal) }\end{array}$ & Rabbit & 15303-1-AP & $\begin{array}{l}\text { Proteintech, } \\
\text { Rosemont, USA }\end{array}$ \\
\hline $\begin{array}{l}\text { human LAMP2 } \\
\text { (polyclonal) }\end{array}$ & Goat & AF6228 & $\begin{array}{l}\text { R\&D Systems, } \\
\text { Minneapolis, USA }\end{array}$ \\
\hline $\begin{array}{l}\text { human LAMP2 } \\
\text { (monoclonal) }\end{array}$ & Mouse & 555803 & $\begin{array}{l}\text { BD Biosciences, } \\
\text { San Jose, USA }\end{array}$ \\
\hline $\begin{array}{l}\text { human p62 } \\
\text { (polyclonal) }\end{array}$ & Guinea pig & GP62-C & $\begin{array}{l}\text { ProGen Biotechnik, } \\
\text { Heidelberg, } \\
\text { Germany }\end{array}$ \\
\hline $\begin{array}{l}\text { human } \beta \text {-Actin } \\
\text { (monoclonal) }\end{array}$ & Mouse & A5316 & $\begin{array}{l}\text { Sigma Aldrich, } \\
\text { Hamburg, } \\
\text { Germany }\end{array}$ \\
\hline
\end{tabular}




\subsubsection{Secondary antibodies}

\begin{tabular}{|c|c|c|c|c|}
\hline Reactivity & Host & Conjugate & Cat. No. & Supplier \\
\hline Goat IgG & Donkey & $\begin{array}{l}\text { AlexaFluor® } \\
633\end{array}$ & A-21082 & $\begin{array}{l}\text { Thermo- } \\
\text { Scientific, } \\
\text { Braunschweig, } \\
\text { Germany }\end{array}$ \\
\hline $\begin{array}{l}\text { Guinea pig } \\
\text { IgG }\end{array}$ & Donkey & $\begin{array}{l}\text { IRDye® } \\
800 C W\end{array}$ & $926-32411$ & $\begin{array}{l}\text { Li-cor } \\
\text { Biosciences } \\
\text { GmbH, Bad } \\
\text { Homburg, } \\
\text { Germany }\end{array}$ \\
\hline Mouse IgG & Donkey & $\begin{array}{l}\text { AlexaFluor }{ }^{\circledR} \\
488\end{array}$ & A-21202 & $\begin{array}{l}\text { Thermo- } \\
\text { Scientific, } \\
\text { Braunschweig, } \\
\text { Germany }\end{array}$ \\
\hline Mouse IgG & Donkey & $\begin{array}{l}\text { AlexaFluor® } \\
546\end{array}$ & A-10036 & $\begin{array}{l}\text { Thermo- } \\
\text { Scientific, } \\
\text { Braunschweig, } \\
\text { Germany }\end{array}$ \\
\hline Mouse IgG & Donkey & $\begin{array}{l}\text { IRDye® } \\
\text { 680RD }\end{array}$ & $926-68072$ & $\begin{array}{l}\text { Li-cor } \\
\text { Biosciences } \\
\text { GmbH, Bad } \\
\text { Homburg, } \\
\text { Germany }\end{array}$ \\
\hline Mouse IgG & Donkey & $\begin{array}{l}\text { IRDye® } \\
800 C W\end{array}$ & $926-32212$ & $\begin{array}{l}\text { Li-cor } \\
\text { Biosciences } \\
\text { GmbH, Bad } \\
\text { Homburg, } \\
\text { Germany }\end{array}$ \\
\hline Rabbit IgG & Donkey & $\begin{array}{l}\text { AlexaFluor }{ }^{\circledR} \\
488\end{array}$ & A-21206 & $\begin{array}{l}\text { Thermo- } \\
\text { Scientific, } \\
\text { Braunschweig, } \\
\text { Germany }\end{array}$ \\
\hline Rabbit IgG & Donkey & $\begin{array}{l}\text { AlexaFluor® } \\
546\end{array}$ & A-10040 & $\begin{array}{l}\text { Thermo- } \\
\text { Scientific, } \\
\text { Braunschweig, } \\
\text { Germany }\end{array}$ \\
\hline Rabbit IgG & Donkey & $\begin{array}{l}\text { IRDye® } \\
\text { 680RD }\end{array}$ & $926-68073$ & $\begin{array}{l}\text { Li-cor } \\
\text { Biosciences } \\
\text { GmbH, Bad } \\
\text { Homburg, } \\
\text { Germany }\end{array}$ \\
\hline
\end{tabular}




\begin{tabular}{|c|c|c|c|c|}
\hline \multirow[t]{3}{*}{ Rabbit IgG } & \multirow[t]{3}{*}{ Donkey } & \multirow{3}{*}{$\begin{array}{l}\text { IRDye® } \\
800 C W\end{array}$} & \multirow[t]{3}{*}{$926-32213$} & Li-cor \\
\hline & & & & Biosciences \\
\hline & & & & $\begin{array}{l}\text { GmbH, Bad } \\
\text { Homburg, } \\
\text { Germany }\end{array}$ \\
\hline Rabbit IgG & Donkey & $\begin{array}{l}\text { Horseradish } \\
\text { peroxidase }\end{array}$ & NA934 & $\begin{array}{l}\text { Sigma Aldrich, } \\
\text { Hamburg, } \\
\text { Germany }\end{array}$ \\
\hline
\end{tabular}

3.4. Oligonucleotides

\begin{tabular}{|c|c|c|}
\hline Target gene & Transcript ID & Sequence \\
\hline $\begin{array}{l}\text { control siRNA-A } \\
\text { (SantaCruz } \\
\text { Biotechnology, } \\
\text { Heidelberg, Germany) }\end{array}$ & - & - \\
\hline $\begin{array}{l}\text { hABCA1 } \\
\text { (forward qPCR primer) }\end{array}$ & ENST00000374736.8 & $\begin{array}{l}5^{\prime}-\text { GCT CGC CTG TTC } \\
\text { TCA GAT GC-3' }\end{array}$ \\
\hline $\begin{array}{l}\text { hABCA1 } \\
\text { (reverse qPCR primer) }\end{array}$ & ENST00000374736.8 & $\begin{array}{l}5^{\prime}-\text { GGA GAA } \\
\text { CAG CCC TCA } \\
\text { GC-3' }\end{array}$ \\
\hline $\begin{array}{l}\text { hAPOE } \\
\text { (forward qPCR primer) }\end{array}$ & ENST00000252486.9 & $\begin{array}{l}5^{\prime}-\mathrm{CCA} \text { ATC } \text { ACA } \text { GGC } \\
\text { AGG AAG ATG AAG G-3' }\end{array}$ \\
\hline $\begin{array}{l}\text { hAPOE } \\
\text { (reverse qPCR primer) }\end{array}$ & ENST00000252486.9 & $\begin{array}{l}5^{\prime}-\mathrm{AGA} \text { CAG TGT CTG } \\
\text { CAC CCA GC-3' }\end{array}$ \\
\hline $\begin{array}{l}\text { hCH25H } \\
\text { (forward qPCR primer) }\end{array}$ & ENST00000371852.4 & $\begin{array}{l}\text { 5'-GGT CCT GGA TAT } \\
\text { CCT GTG CTC } \mathrm{C}-3 \text { ' }\end{array}$ \\
\hline $\begin{array}{l}\text { hCH25H } \\
\text { (reverse qPCR primer) }\end{array}$ & ENST00000371852.4 & $\begin{array}{l}\text { 5'-GAG }^{\prime} \text { TAG } \\
\text { GAA CAG }\end{array}$ \\
\hline $\begin{array}{l}\text { hCYP7B1 } \\
\text { (forward qPCR primer) }\end{array}$ & ENST00000310193.4 & 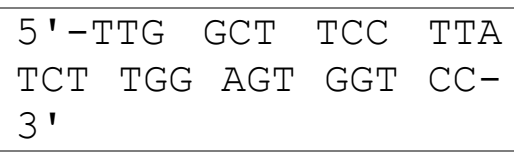 \\
\hline $\begin{array}{l}\text { hCYP7B1 } \\
\text { (reverse qPCR primer) }\end{array}$ & ENST00000310193.4 & 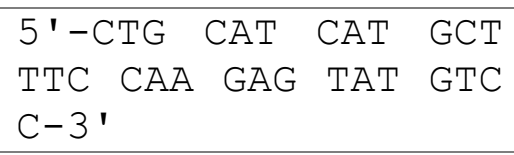 \\
\hline $\begin{array}{l}\text { hGBP1 } \\
\text { (forward qPCR primer) }\end{array}$ & ENST00000370473.5 & $\begin{array}{l}5^{\prime}-\mathrm{GGT} \text { CCA GTT GCT } \\
\text { GAA AGA GC-3' }\end{array}$ \\
\hline $\begin{array}{l}\text { hGBP1 } \\
\text { (reverse qPCR primer) }\end{array}$ & ENST00000370473.5 & $\begin{array}{l}5^{\prime}-\mathrm{TGA} \text { CAG GAA GGC } \\
\text { TCT GGT CT-3' }\end{array}$ \\
\hline $\begin{array}{l}\text { HEV ORF2 } \\
\text { (forward qPCR primer) }\end{array}$ & KC618402.1 & $\begin{array}{l}5^{\prime}-\text { GGT GGT TTC TGG } \\
\text { GGT GAC-3' }\end{array}$ \\
\hline $\begin{array}{l}\text { HEV ORF2 } \\
\text { (reverse qPCR primer) }\end{array}$ & KC618402.1 & $\begin{array}{l}5^{\prime}-A G G \quad \text { GGT } \text { TGG } \\
\text { GAT GA } 3 \text { ' }\end{array}$ \\
\hline
\end{tabular}




\begin{tabular}{|c|c|c|}
\hline $\begin{array}{l}\text { hHMGCR } \\
\text { (forward qPCR primer) }\end{array}$ & ENST00000287936.9 & $\begin{array}{llll}5^{\prime}-\mathrm{CCT} & \text { TAG } & \text { TGG } & \text { CTG } \\
\text { AAA CAG } & \text { ATA } & \text { CCC }-3^{\prime}\end{array}$ \\
\hline $\begin{array}{l}\text { hHMGCR } \\
\text { (reverse qPCR primer) }\end{array}$ & ENST00000287936.9 & $\begin{array}{l}5^{\prime}-\mathrm{CTG} \text { GAT GAT CTC } \\
\text { AGC ATC ACT AAG G-3' }\end{array}$ \\
\hline $\begin{array}{l}\text { hNR1H4/FXR } \\
\text { (forward qPCR primer) }\end{array}$ & ENST00000392986.8 & $\begin{array}{l}5^{\prime}-\mathrm{CAT} \text { GCG } \text { AAG AAA } \\
\text { GTG TCA AGA } \text { GTG TCG- } \\
3\end{array}$ \\
\hline $\begin{array}{l}\text { hNR1H4/FXR } \\
\text { (reverse qPCR primer) }\end{array}$ & ENST00000392986.8 & $\begin{array}{l}\text { 5'-CCT GCA TGA CTT } \\
\text { TGT TGT CGA GG-3' }\end{array}$ \\
\hline $\begin{array}{l}\text { hPCSK9 } \\
\text { (forward qPCR primer) }\end{array}$ & ENST00000302118.5 & $\begin{array}{l}5^{\prime}-\mathrm{GCC} \text { AGG ACA GCA } \\
\text { ACC TCT CC-3' }\end{array}$ \\
\hline $\begin{array}{l}\text { hPCSK9 } \\
\text { (reverse qPCR primer) }\end{array}$ & ENST00000302118.5 & $\begin{array}{l}5^{\prime}-\mathrm{TTC} \text { AGC } \text { ACC } \mathrm{ACC} \\
\text { ACG TAG GTG } \mathrm{C}-3^{\prime}\end{array}$ \\
\hline $\begin{array}{l}\text { hRPL27 } \\
\text { (forward qPCR primer) }\end{array}$ & ENST00000253788.12 & $\begin{array}{l}5^{\prime}-\mathrm{AAA} \text { GCT GTC ATC } \\
\text { GTG AAG AAC-3' }\end{array}$ \\
\hline $\begin{array}{l}\text { hRPL27 } \\
\text { (reverse qPCR primer) }\end{array}$ & ENST00000253788.12 & $\begin{array}{l}5^{\prime}-\mathrm{GCT} \text { GCT ACT } \\
\text { CGG GGG TAG-3' }\end{array}$ \\
\hline $\begin{array}{l}\text { hGBP1 } \\
\text { (sense siRNA) }\end{array}$ & ENST00000370473.5 & $\begin{array}{l}5^{\prime}-\mathrm{GGC} \text { AUG UAC CAU } \\
\text { AAG CUA AAG ACC A-3' }\end{array}$ \\
\hline $\begin{array}{l}\text { hGBP1 } \\
\text { (antisense siRNA) }\end{array}$ & ENST00000370473.5 & $\begin{array}{l}5^{\prime}-\text { UGG UCU UUA GCU } \\
\text { UAU GGU ACA UGC CUU- } \\
3^{\prime}\end{array}$ \\
\hline $\begin{array}{l}\text { Random } \\
\text { primers }\end{array}$ & - & - \\
\hline
\end{tabular}

\subsection{Plasmid DNA}

\begin{tabular}{lll}
\hline Plasmid name & Description & Supplier \\
\hline pCMV2b-tag-GBP1- & Encoding human GBP1 Dr. Gerrit J.K. Praefcke \\
R48A & with R48A mutation \\
& under control of CMV- \\
& promoter \\
\hline pCMV2b-tag-GBP1-S73A & Encoding human GBP1 Dr. Gerrit J.K. Praefcke \\
& with S73A mutation under \\
& control of CMV-promoter \\
\hline pCMV2b-tag-GBP1-wt & Encoding human wild- Dr. Gerrit J.K. Praefcke \\
& type GBP1 under control \\
& of CMV-promoter
\end{tabular}




\begin{tabular}{|c|c|c|}
\hline pGL3-GBP1-Luc & $\begin{array}{l}\text { Encoding firefly luciferase } \\
\text { under endogenous, } \\
\text { proximal human GBP1- } \\
\text { promoter }\end{array}$ & Dr. Xingjian Wen \\
\hline pGL3-GAS-Luc & $\begin{array}{l}\text { Encoding firefly luciferase } \\
\text { under gamma-activated- } \\
\text { site-driven promoter }\end{array}$ & Cloned internally \\
\hline pGL3-ISRE-Luc & $\begin{array}{l}\text { Encoding firefly luciferase } \\
\text { under interferon- } \\
\text { stimulated-element- } \\
\text { driven promoter }\end{array}$ & Cloned internally \\
\hline pmCherry & $\begin{array}{l}\text { Encoding mCherry under } \\
\text { control of CMV-promoter }\end{array}$ & Cloned internally \\
\hline
\end{tabular}

3.6. Organisms

\subsubsection{Eukaryotic cells}

\begin{tabular}{lll}
\hline Organism & Type & Supplier \\
\hline A549/D3 & A549 subclone & Prof. Reimar Johne \\
\hline A549/N5 & Persistently HEV-infected & Prof. Reimar Johne \\
& A549 (gt3c, strain 47832c) & \\
\hline
\end{tabular}

\subsubsection{Viruses}

\begin{tabular}{llll}
\hline Organism & Strain & Genotype & Supplier \\
\hline Hepatitis E virus & $47832 \mathrm{c}$ & $3 \mathrm{c}$ & Prof. Reimar Johne \\
\hline
\end{tabular}

\subsection{Software}

\begin{tabular}{ll}
\hline Software & Supplier \\
\hline Citavi 5 & Swiss Academic Software GmbH, CH \\
\hline FIJI & Open source \\
\hline GraphPad Prism 8 & GraphPad, USA \\
\hline Image Studio Lite & LI-COR Biosciences, Lincoln, US \\
\hline LAS X Control Software & Leica, Wetzlar, Germany \\
\hline LAS X core offline & Leica, Wetzlar, Germany \\
\hline LightCycler 480 SW1.5.1 & Roche Diagnostics, Mannheim, Germany \\
\hline MS Office & Microsoft, USA \\
\hline TECAN iControl & Tecan, Switzerland \\
\hline
\end{tabular}




\subsection{Devices}

\begin{tabular}{|c|c|c|}
\hline Device & Function & Supplier \\
\hline Dmi8 microscope & Microscope & Leica, Wetzlar, Germany \\
\hline $\begin{array}{l}\text { Electrophoresis } \\
\text { supply EPS } 301\end{array}$ & Power supply & $\begin{array}{l}\text { GE Healthcare, Freiburg, } \\
\text { Germany }\end{array}$ \\
\hline Heraeus Multifuge 1S-R & Centrifuge & $\begin{array}{l}\text { Thermo-Scientific, } \\
\text { Braunschweig, Germany }\end{array}$ \\
\hline $\begin{array}{l}\text { IKAMAG® universal hot } \\
\text { plate magnetic stirrer }\end{array}$ & Stirrer & IKA, Staufen, Germany \\
\hline Incubator BBD 6220 & Incubator & $\begin{array}{l}\text { Heraeus, OsteroGermany, } \\
\text { Germany }\end{array}$ \\
\hline Infinite M1000 & Microplate reader & Tecan, Switzerland \\
\hline JA25.50 & Centrifuge rotor & $\begin{array}{l}\text { Beckmann Coulter, } \\
\text { Krefeld, Germany }\end{array}$ \\
\hline Leica TCS SP8 System & $\begin{array}{l}\text { Confocal laser scanning } \\
\text { microscope system }\end{array}$ & Leica, Wetzlar, Germany \\
\hline LI-COR Odyssey & Infrared imager & $\begin{array}{l}\text { LI-COR } \\
\text { Lincoln, US }\end{array}$ \\
\hline $\begin{array}{l}\text { LightCycler } \\
\text { Instrument II }\end{array}$ & qPCR device & $\begin{array}{l}\text { Roche Diagnostics, } \\
\text { Mannheim, Germany }\end{array}$ \\
\hline $\begin{array}{l}\text { Mighty small II vertical } \\
\text { electrophoresis } \\
\text { SE250 }\end{array}$ & Electrophoresis chamber & $\begin{array}{l}\text { GE Healthcare, Freiburg, } \\
\text { Germany }\end{array}$ \\
\hline $\begin{array}{l}\text { Mighty small multiple } \\
\text { gel caster SE } 200\end{array}$ & Gel caster & $\begin{array}{l}\text { GE Healthcare, Freiburg, } \\
\text { Germany }\end{array}$ \\
\hline Nanophotometer P300 & Photometer & $\begin{array}{l}\text { Implen, München, } \\
\text { Germany }\end{array}$ \\
\hline $\begin{array}{l}\text { Optima }^{\mathrm{TM}} \quad \mathrm{L}-80 \quad \mathrm{XP} \\
\text { Preparative } \\
\text { Ultracentrifuge }\end{array}$ & Ultracentrifuge & $\begin{array}{l}\text { Beckmann Coulter, } \\
\text { Krefeld, Germany }\end{array}$ \\
\hline Optima $^{\mathrm{TM}}$ Max XP & Ultracentrifuge & $\begin{array}{l}\text { Beckmann Coulter, } \\
\text { Krefeld, Germany }\end{array}$ \\
\hline $\begin{array}{l}\text { Orion II Microplate } \\
\text { Luminometer }\end{array}$ & Microplate reader & $\begin{array}{l}\text { Titertek, Pforzheim, } \\
\text { Germany }\end{array}$ \\
\hline Pipettes & & $\begin{array}{l}\text { Eppendorf, } \\
\text { Germany }\end{array}$ \\
\hline Rocking platform WT16 & Roller & $\begin{array}{l}\text { Biometra, } \\
\text { Germany }\end{array}$ \\
\hline S20 - SevenEasy ${ }^{\mathrm{TM}} \mathrm{pH}$ & $\mathrm{pH}$ probe & Mettler Toledo, Germany \\
\hline
\end{tabular}




\begin{tabular}{|c|c|c|}
\hline $\begin{array}{l}\text { Satorius balance LP } 6000 \\
200 S\end{array}$ & Scale & $\begin{array}{l}\text { Satorius, Göttingen, } \\
\text { Germany }\end{array}$ \\
\hline SonoPuls MS73 & Sonicator & $\begin{array}{l}\text { BanGermanylin, Berlin, } \\
\text { Germany }\end{array}$ \\
\hline SW32-Ti & Ultracentrifuge rotor & $\begin{array}{l}\text { Beckmann Coulter, } \\
\text { Krefeld, Germany }\end{array}$ \\
\hline SW41-Ti & Ultracentrifuge rotor & $\begin{array}{l}\text { Beckmann Coulter, } \\
\text { Krefeld, Germany }\end{array}$ \\
\hline $\begin{array}{l}\text { TE77 ECL semi dry } \\
\text { transfer unit }\end{array}$ & Blotter & $\begin{array}{l}\text { GE Healthcare, Freiburg, } \\
\text { Germany }\end{array}$ \\
\hline Thermomixer compact & Heat block & $\begin{array}{l}\text { Eppendorf, Hamburg, } \\
\text { Germany }\end{array}$ \\
\hline TLS-55 & Ultracentrifuge rotor & $\begin{array}{l}\text { Beckmann Coulter, } \\
\text { Krefeld, Germany }\end{array}$ \\
\hline Vortex®Genie 2 & Agitation platform & Scientific Industries, US \\
\hline Water bath1228-2F & Water bath & $\begin{array}{l}\text { VWR, Darmstadt, } \\
\text { Germany }\end{array}$ \\
\hline
\end{tabular}

\subsection{Consumables}

\begin{tabular}{ll}
\hline Consumable & Supplier \\
\hline Cell culture flasks & Greiner, Frickenhausen, Germany \\
\hline Cell scrapers & Greiner, Frickenhausen, Germany \\
\hline Coverslips & Carl Roth, Karlsruhe, Germany \\
\hline Falcon tubes & Greiner, Frickenhausen, Germany \\
\hline Filtered pipette tips & 4titude, Berlin, Germany \\
\hline Glass coverslips & Carl Roth, Karlsruhe, Germany \\
\hline Glass slides & Carl Roth, Karlsruhe, Germany \\
\hline Hybond-P PVDF membrane & Millipore, Schwalbach, Germany \\
\hline LightCycler Multiwell plates & Genaxxon, Biberach, Germany \\
\hline Multiwell plates & Greiner, Frickenhausen, Germany \\
\hline Page Ruler Prestained protein ladder & Thermo-Scientific, \\
\hline $\begin{array}{l}\text { RotiLabo }{ }^{\circledR} \text { syringe filters } \mathbf{( 0 , 2 2 ~ / ~ 0 , 4 5} \\
\text { Mm) }\end{array}$ & Germany \\
\hline Safe-lock micro test tubes & Sarstedt, Nümbrecht, Germany \\
\hline Syrological pipettes & Greiner, Frickenhausen, Germany \\
\hline Ultracentrifugation tubes & Beckmann Coulter, Krefeld, Germany \\
\hline Whatman filter paper & GE Healthcare, Freiburg, Germany \\
\hline &
\end{tabular}




\subsection{Kits}

\begin{tabular}{|c|c|}
\hline Kit & Supplier \\
\hline LDH Cytotoxicity Detection Kit & Clontech, Mountain View, US \\
\hline $\begin{array}{l}\text { LightCycler Multiplex RNA Master } \\
\text { Mix }\end{array}$ & Roche Diagnostics, Mannheim, Germany \\
\hline $\begin{array}{l}\text { LightMix Modular Hepatitis E Virus } \\
\text { Kit }\end{array}$ & TIB Molbio, Berlin, Germany \\
\hline $\begin{array}{l}\text { Lipofectamine }{ }^{\mathrm{TM}} \quad 3000 \quad \text { Transfection } \\
\text { Reagent }\end{array}$ & $\begin{array}{l}\text { Thermo-Scientific, } \\
\text { Germany }\end{array}$ \\
\hline LysoTracker ${ }^{\mathrm{TM}}$ Red DND-99 & $\begin{array}{l}\text { Thermo-Scientific, } \\
\text { Germany }\end{array}$ \\
\hline Maxima SYBR-Green qPCR Kit & $\begin{array}{l}\text { Thermo-Scientific, } \\
\text { Germany }\end{array}$ \\
\hline $\begin{array}{lll}\text { PageRulerTM } & \text { Prestained } & \text { Protein } \\
\text { Ladder } & & \end{array}$ & $\begin{array}{l}\text { Thermo-Scientific, } \\
\text { Germany }\end{array}$ \\
\hline PrestoBlue $^{\mathrm{TM}}$ Cell Viability Reagent & $\begin{array}{l}\text { Thermo-Scientific, } \\
\text { Germany }\end{array}$ \\
\hline RNase-Free DNase Set & QIAGEN, Hilden, Germany \\
\hline RNeasy Mini Kit & QIAGEN, Hilden, Germany \\
\hline siPORT ${ }^{\mathrm{TM}}$ NeoFX ${ }^{\mathrm{TM}}$ Transfection Agent & $\begin{array}{l}\text { Thermo-Scientific, Braunschweig, } \\
\text { Germany }\end{array}$ \\
\hline
\end{tabular}

\subsection{Chemicals}

\subsubsection{Generic chemicals}

\begin{tabular}{lll}
\hline Chemical & Abbreviation & Supplier \\
\hline 1,4-diazabicyclo[2.2.2]octane & DABCO & $\begin{array}{l}\text { Merck. Darmstadt, } \\
\text { Germany }\end{array}$ \\
\hline $\begin{array}{l}\text { 2-Amino-2-(hydroxymethyl) } \\
\text { propan-1,3-diol }\end{array}$ & Tris & $\begin{array}{l}\text { Carl Roth, Karlsruhe, } \\
\text { Germany }\end{array}$ \\
\hline 2-mercaptoethanol & & $\begin{array}{l}\text { Carl Roth, Karlsruhe, } \\
\text { Germany }\end{array}$ \\
\hline 3-Amino-9-ethylcarbazole & Carbazole & $\begin{array}{l}\text { Carl Roth, Karlsruhe, } \\
\text { Germany }\end{array}$ \\
\hline $\begin{array}{l}\text { 4',6-diamidino-2- } \\
\text { phenylindole }\end{array}$ & DAPI & $\begin{array}{l}\text { Sigma-Aldrich, } \\
\text { Hamburg, Germany }\end{array}$ \\
\hline
\end{tabular}




\begin{tabular}{|c|c|c|}
\hline 6-Aminohexanoic acid & & $\begin{array}{l}\text { Carl Roth, Karlsruhe, } \\
\text { Germany }\end{array}$ \\
\hline Acetic acid (glacial) & & $\begin{array}{l}\text { Carl Roth, Karlsruhe, } \\
\text { Germany }\end{array}$ \\
\hline Acetone & & $\begin{array}{l}\text { Carl Roth, Karlsruhe, } \\
\text { Germany }\end{array}$ \\
\hline Ammonium persulfate & APS & $\begin{array}{l}\text { Carl Roth, Karlsruhe, } \\
\text { Germany }\end{array}$ \\
\hline Bradford reagent & & $\begin{array}{l}\text { Sigma Aldrich, } \\
\text { Hamburg, Germany }\end{array}$ \\
\hline Bromophenole blue & & $\begin{array}{l}\text { Merck, Darmstadt, } \\
\text { Germany }\end{array}$ \\
\hline Chloroform & & $\begin{array}{l}\text { Carl Roth, Karlsruhe, } \\
\text { Germany }\end{array}$ \\
\hline $\begin{array}{l}\text { Deoxynucleoside } \\
\text { triphosphates }\end{array}$ & dNTPs & $\begin{array}{l}\text { Thermo-Scientific, } \\
\text { Braunschweig, } \\
\text { Germany }\end{array}$ \\
\hline Diethyl pyrocarbonate & DEPC & $\begin{array}{l}\text { Paul-Ehrlich-Institut, } \\
\text { Langen, Germany }\end{array}$ \\
\hline Dimethyl sulfoxide & DMSO & $\begin{array}{l}\text { Genaxxon, Biberach, } \\
\text { Germany }\end{array}$ \\
\hline Double distilled water & $\mathrm{ddH}_{2} \mathrm{O}$ & $\begin{array}{l}\text { Paul-Ehrlich-Institut, } \\
\text { Langen, Germany }\end{array}$ \\
\hline $\begin{array}{l}\text { Ethanol (96 } \% \text { v/v, non- } \\
\text { denatured) }\end{array}$ & & $\begin{array}{l}\text { Carl Roth, Karlsruhe, } \\
\text { Germany }\end{array}$ \\
\hline $\begin{array}{l}\text { Ethylenediaminetetraacetic } \\
\text { acid }\end{array}$ & EDTA & $\begin{array}{l}\text { Serva, Heidelberg, } \\
\text { Germany }\end{array}$ \\
\hline Filipin III & Filipin & $\begin{array}{l}\text { Sigma-Aldrich, } \\
\text { Hamburg, Germany }\end{array}$ \\
\hline Glycerol $99.5 \%$ v/v & & $\begin{array}{l}\text { GERBU Biotechnik, } \\
\text { Gaiberg, Germany }\end{array}$ \\
\hline Hydrogen peroxide & $\mathrm{H}_{2} \mathrm{O}_{2}$ & $\begin{array}{l}\text { Carl Roth, Karlsruhe, } \\
\text { Germany }\end{array}$ \\
\hline Iodixanol (OptiPrep ${ }^{\mathrm{TM}}$ ) & & $\begin{array}{l}\text { Sigma-Aldrich, } \\
\text { Hamburg, Germany }\end{array}$ \\
\hline Isopropanol & & $\begin{array}{l}\text { Carl Roth, Karlsruhe, } \\
\text { Germany }\end{array}$ \\
\hline L-glutamine & & PAA, Linz, Austria \\
\hline Methanol (96 \% v/v) & & $\begin{array}{l}\text { Carl Roth, Karlsruhe, } \\
\text { Germany }\end{array}$ \\
\hline Mowiol & & $\begin{array}{l}\text { Sigma-Aldrich, } \\
\text { Hamburg, Germany }\end{array}$ \\
\hline
\end{tabular}




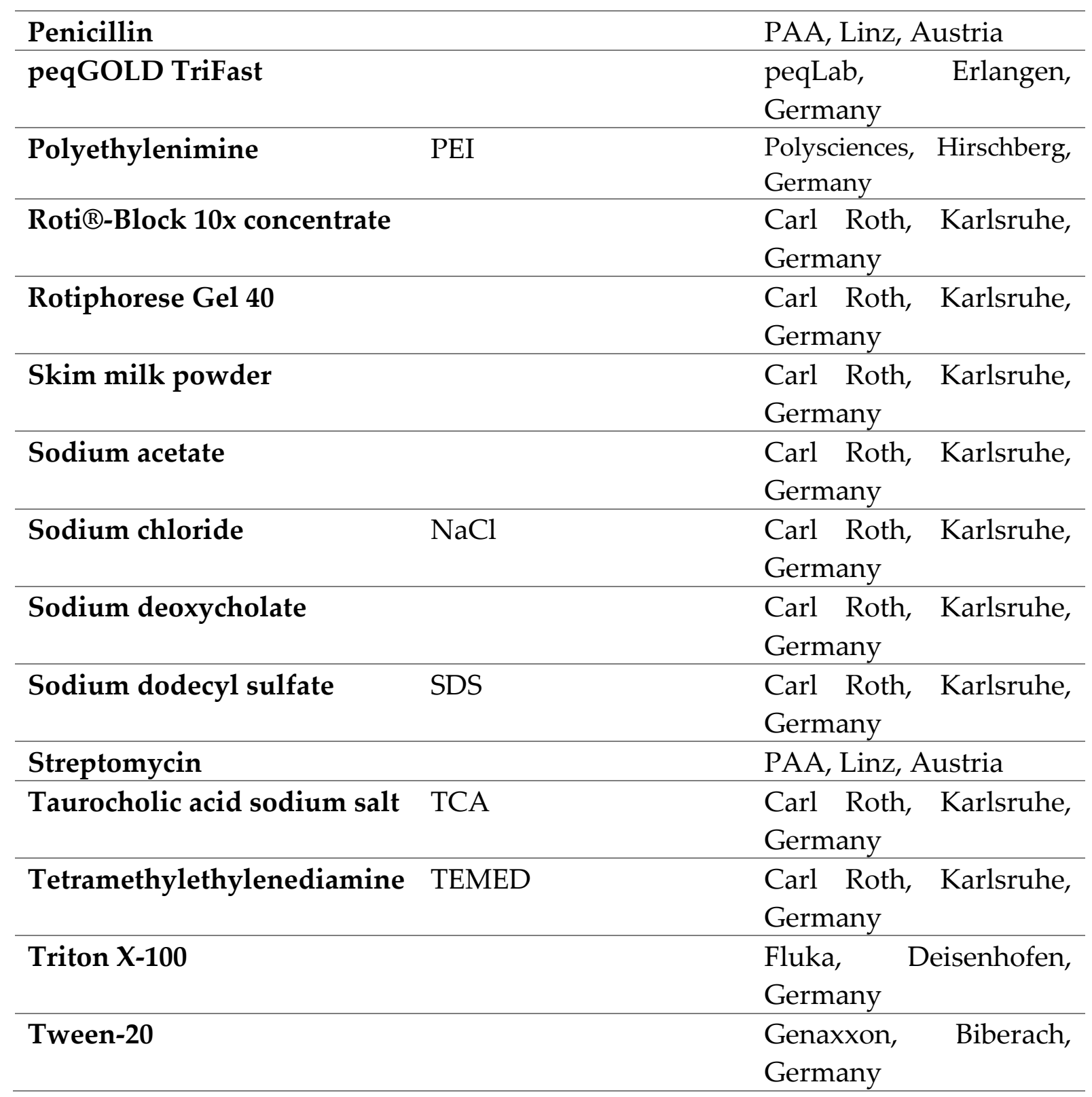

\subsubsection{Chemicals used for treatments}

\begin{tabular}{lll}
\hline Chemical & Abbreviation & Supplier \\
\hline 25-hydroxycholesterol & 25-HC & $\begin{array}{l}\text { Cayman Chemicals, Ann } \\
\text { Arbor, USA }\end{array}$ \\
\hline Avasimibe & & $\begin{array}{l}\text { SantaCruz Biotechnology, } \\
\text { Heidelberg, Germany }\end{array}$ \\
\hline Cycloheximide & CHX & Sigma Aldrich, Hamburg, \\
& & Germany \\
\hline Fenofibrate & SantaCruz Biotechnology, \\
& & Heidelberg, Germany \\
\hline Gemfibrozil & SantaCruz Biotechnology, \\
& & Heidelberg, Germany \\
\hline
\end{tabular}




\begin{tabular}{ll}
\hline Leupeptin & $\begin{array}{l}\text { Sigma Aldrich, Hamburg, } \\
\text { Germany }\end{array}$ \\
\hline PSC833 & SantaCruz Biotechnology, \\
& Heidelberg, Germany \\
\hline Simvastatin & SantaCruz Biotechnology, \\
& Heidelberg, Germany \\
\hline
\end{tabular}

\subsection{Proteins and protein complexes}

\begin{tabular}{|c|c|}
\hline Protein & Supplier \\
\hline Alirocumab & Creative Biolabs, Shirley, USA \\
\hline Bovine serum albumin & PAA, Linz, Austria \\
\hline FGF19 & Peprotech, Hamburg, Germany \\
\hline Interferon alpha & Peprotech, Hamburg, Germany \\
\hline Interferon beta & Peprotech, Hamburg, Germany \\
\hline Interferon gamma & Peprotech, Hamburg, Germany \\
\hline LDL & Lee Biosolutions, Metro Ct, USA \\
\hline $\begin{array}{l}\text { Reverted Aid } \text { AM }^{\mathrm{TM}} \text { Minus Reverse } \\
\text { Transcriptase }(200 \mathrm{U} / \mu \mathrm{L})\end{array}$ & $\begin{array}{l}\text { Thermo Scientific, Braunschweig, } \\
\text { Germany }\end{array}$ \\
\hline RQ1 RNase-Free DNase & Promega GmbH, Walldorf, Germany \\
\hline Trypsin & Paul-Ehrlich-Institut, Langen, Germany \\
\hline
\end{tabular}




\section{Methods}

\subsection{General cell culture}

In this study, A549 cells (ATCC $®$ CCL-185 ${ }^{\mathrm{TM}}$ ), a human lung carcinoma-derived cell line, were used as a model system for in vitro studies of HEV. Initially, these cells were isolated from a 58-year-old Caucasian male and display an epithelial-like, adherent morphology [394]. Subsequently, these cells were sub-cloned for high susceptibility to an HEV-infection, yielding HEV-permissive A549/D3 cells [395]. On the other hand, parental A549 cells were inoculated with an HEV genotype 3c isolate, termed isolate 47832c (GenBank: KC618402.1), obtained from a chronically infected renal transplant patient. Resulting HEV-infected A549 cells retained viral infection after passaging and low-temperature storage, effectively yielding a persistent infection and are therefore referred to as persistently HEV-infected A549 cells (or A549/N5 cells) [23]. It must be noted that, even though HEV virions are always present in culture media of A549/N5 cells, not each cell within this culture is infected.

Cells were cultivated in Dulbecco's Modified Eagle's Medium (DMEM, $4.5 \mathrm{~g} / \mathrm{L}$ glucose) supplemented with L-glutamine $(2 \mathrm{mM})$, streptomycin $(100 \mu \mathrm{g} / \mathrm{mL})$, penicillin $(100 \mathrm{IU} / \mathrm{mL})$ and foetal bovine serum $(10 \% \mathrm{v} / \mathrm{v})$. Cultivation was performed at $37{ }^{\circ} \mathrm{C}$ with $95 \%$ humidity and $5 \%$ atmospheric $\mathrm{CO}_{2}$ to maintain the $\mathrm{pH}$-value of the medium. Passaging of cells was performed every 3-4 days while keeping cells as confluent as possible, which, empirically determined, yielded the highest number of HEV-infected cells within the culture. Unless stated otherwise, the following cell densities were used throughout the experiments in respective multiwell-plates: (i) $3 \times 10^{5}$ cells/well (6-well-plate), (ii) $1 \times 10^{5}$ cells/well (12-well-plate) or (iii) $1 \times 10^{4}$ cells/well (96-well-plate).

\subsection{Generation of infection stocks}

To generate crudely purified HEV virions, A549/N5 cells were grown in T175 cell culture flasks with $30 \mathrm{~mL}$ growth medium for 14 days. Subsequently, cell culture supernatant of five T175 cell culture flasks was collected and centrifuged $10 \mathrm{~min}$ at $1,000 \mathrm{xg}$ at $4{ }^{\circ} \mathrm{C}$ to rid the solution of cell debris. The resulting supernatant was further centrifuged $30 \mathrm{~min}$ at $20,000 \mathrm{xg}$ at $4{ }^{\circ} \mathrm{C}$ in a JA25.50 rotor as a second pre-purification. Virions were subsequently pelleted from this solution via centrifugation for $1.5 \mathrm{~h}$ at $125.000 \mathrm{xg}$ at $4{ }^{\circ} \mathrm{C}$ in an SW-32Ti rotor with the supernatant being discarded afterwards. The pellet containing virions was resuspended in a final volume of $5 \mathrm{~mL}$ PBS supplemented with $125 \mathrm{mM}$ taurocholic acid and incubated $1.5 \mathrm{~h}$ at $37^{\circ} \mathrm{C}$ under agitation to remove the quasi-envelope from eHEV virions, consequently yielding $\mathrm{nHEV}$ virions. As a last purification step, the $\mathrm{nHEV}$ virion-containing solution was 
layered onto a discontinuous density-gradient $\left(60 \% \mathrm{w} / \mathrm{v}\right.$ Iodixanol in $\mathrm{ddH}_{2} \mathrm{O}$, $30 \% \mathrm{w} / \mathrm{v}$ Iodixanol in $\mathrm{ddH}_{2} \mathrm{O}$ and $10 \% \mathrm{w} / \mathrm{v}$ Iodixanol in $\mathrm{ddH}_{2} \mathrm{O}$ from bottom to top) and centrifuged $4 \mathrm{~h}$ at $287,400 \mathrm{xg}$ at $4{ }^{\circ} \mathrm{C}$ in an SW41-Ti rotor to achieve isopycnic centrifugation. As nHEV virions $(\mathrm{Q}=1.18-1.26 \mathrm{~g} / \mathrm{mL})$ band between $30 \% \mathrm{w} / \mathrm{v}$ Iodixanol in $\mathrm{ddH}_{2} \mathrm{O}(\mathrm{Q}=1.16 \mathrm{~g} / \mathrm{mL})$ and $60 \% \mathrm{w} / \mathrm{v}$ Iodixanol in $\mathrm{ddH}_{2} \mathrm{O}(\mathrm{Q}=1.32 \mathrm{~g} / \mathrm{mL})$, $\sim 1 \mathrm{~mL}$ of the interphase between these fractions was extracted using a blunt syringe. The number of viral genomes present in this fraction was assessed via RT-qPCR. Glycerol was added to a final concentration of $50 \% \mathrm{v} / \mathrm{v}$ to allow storage at $-20{ }^{\circ} \mathrm{C}$. If needed, HEV stocks were inactivated by exposure to UV light for $45 \mathrm{~min}$.

\subsection{Transient transfection}

\subsubsection{Overexpression of proteins}

Cells were seeded in growth medium in either 12-well-plates or 6-well-plates with a cell density of $2.5 \times 10^{5}$ cells/well or $5 \times 10^{5}$ cells/well, respectively, and cultivated overnight. The next day, cells were transfected with $2.5 \mu \mathrm{g}$ DNA (6-well-plate) or $1.25 \mu$ g DNA (12-well-plate) per well in combination with Lipofectamine ${ }^{\mathrm{TM}} 3000$ according to the manufacturer's instructions. Transfection mixes were added dropwise to the growth medium. After $24 \mathrm{~h}$ of cultivation, growth media were refreshed and/or treatment was applied for the desired amount of time. Plasmid DNA being used included pCMV2b-tag-GBP1-wt, pCMV2b-tag-GBP1-R48A, pCMV2b-tagGBP1-S73A or pmCherry. Successful transfection was controlled via presence of red fluorescence in pmCherry-transfected cells, which was visible as early as $\sim 16 \mathrm{~h}$ posttransfection or via immunofluorescence microscopy.

\subsubsection{Luciferase reporter assays}

Cells were seeded in 6-well-plates and cultivated overnight. The next day, cells were transfected with $1 \mu \mathrm{g}$ DNA diluted in $200 \mu \mathrm{L}$ PBS supplemented with $6 \mu \mathrm{g}$ polyethylenimine per well. Transfection mixes were incubated $10 \mathrm{~min}$ at RT and added dropwise into the growth medium. After $24 \mathrm{~h}$ of cultivation, growth media were refreshed and/or treatment was applied for the desired amount of time. Plasmid DNA being used included pGL3-GBP1-Luc [396], pGL3-ISRE-Luc or pGL3-GAS-Luc. Successful transfection was controlled via presence of luciferase-activity being measurable in cell lysates. 


\subsection{3. siRNA-mediated silencing}

To achieve an siRNA-mediated knockdown, siPORT ${ }^{\mathrm{TM}}$ NeoFX ${ }^{\mathrm{TM}}$ Transfection Agentbased transfection mixes were prepared to contain a final concentration of $10 \mathrm{nM}$ siRNA in the resulting growth medium according to the manufacturer's instructions. Transfection mixes were dispersed in 12-well-plates and $1 \mathrm{~mL}$ of cell suspension was seeded onto the solution. Cells were cultivated for $48 \mathrm{~h}$ before treatments were applied for the desired amount of time. During the experiments, no medium exchange was performed to ensure cells were always exposed to siRNA, which included siGBP1 [397] or scrambled siRNA (control siRNA-A). Successful transfection was controlled via immunofluorescence microscopy and Western blot analyses.

\subsection{Treatments}

\subsubsection{Stimulation with Interferons}

Transfected cells were treated $24 \mathrm{~h}$ post-transfection with growth medium supplemented with a final concentration of $100 \mathrm{IU} / \mathrm{mL} \operatorname{IFN} \alpha, \operatorname{IFN} \beta$ or $\operatorname{IFN} \gamma$ (reconstituted in PBS) for a duration of $24 \mathrm{~h}$. A medium exchange was performed for all plasmid DNA-based transfections to start the treatment. Treatment of GBP1silenced cells and respective control transfections was achieved by addition of a prediluted Interferon stock $(4,000 \mathrm{IU} / \mathrm{mL}$ in growth medium) to the growth medium present in respective wells, yielding a final concentration of $100 \mathrm{IU} / \mathrm{mL} \mathrm{IFN} \gamma$. Untransfected cells were treated $48 \mathrm{~h}$ post-seeding with growth medium supplemented with a final concentration of $100 \mathrm{IU} / \mathrm{mL} \operatorname{IFN} \alpha, \operatorname{IFN} \beta$ or $\operatorname{IFN} \gamma$ (reconstituted in PBS) for a duration of $24 \mathrm{~h}$. Supplementing growth medium with equal volumes of PBS served as treatment for the experimental control.

\subsubsection{CHX-chase assay}

Cells were treated $48 \mathrm{~h}$ post-seeding with growth medium supplemented with a final concentration of $100 \mu \mathrm{g} / \mathrm{mL} \mathrm{CHX} \mathrm{(dissolved} \mathrm{in} \mathrm{ddH}_{2} \mathrm{O}$ ) over the course of $0,15,30,45$, $60,75,90,105,120,150$ or $180 \mathrm{~min}$. A medium exchange was performed for all conditions to start the treatment. Supplementing growth medium with equal volumes of $\mathrm{ddH}_{2} \mathrm{O}$ served as treatment for the experimental control. 


\subsubsection{Elevation or reduction of intracellular cholesterol}

Cells were seeded in growth medium and treated $24 \mathrm{~h}$ post-seeding with serum-free DMEM supplemented with a final concentration of $200-600 \mu \mathrm{g} / \mathrm{mL}$ LDL (present as human serum-fraction), 25-100 $\mu \mathrm{M} 25-\mathrm{HC}$ (dissolved in $96 \% \mathrm{v} / \mathrm{v}$ ethanol) or $0.5-5 \mu \mathrm{M}$ Simvastatin (dissolved in DMSO) for a duration of $24 \mathrm{~h}$ or $48 \mathrm{~h}$. Treatment with 25-HC was carried out in the presence of $2 \% \mathrm{v} / \mathrm{v}$ ethanol. Supplementing serum-free DMEM with equal volumes of PBS, $2 \% \mathrm{v} / \mathrm{v}$ ethanol or $0.1 \% \mathrm{v} / \mathrm{v}$ DMSO served as treatment for the experimental controls, respectively.

\subsubsection{Cholesterol-modulation via drugs}

Cells were treated $24 \mathrm{~h}$ post-seeding with growth medium supplemented with a final concentration of 2-1250 $\mu \mathrm{M}$ Gemfibrozil (dissolved in DMSO), 1-625 $\mu \mathrm{M}$ Fenofibrate (dissolved in DMSO), 0.01-6.25 $\mu \mathrm{M}$ Avasimibe (dissolved in DMSO), 0.08-50 $\mu \mathrm{M}$ PSC833 (dissolved in DMSO), 0.004-2.5 $\mu \mathrm{g} / \mathrm{mL} \mathrm{FGF19} \mathrm{(reconstituted} \mathrm{in} \mathrm{TBS)} \mathrm{or} \mathrm{0.8-}$ $500 \mathrm{ng} / \mathrm{mL}$ Alirocumab (reconstituted in TBS) for a duration of 24-72 h. Supplementing growth medium with $1 \% \mathrm{v} / \mathrm{v}$ DMSO or equal volumes of TBS served as treatment for the experimental controls, respectively.

\subsubsection{Inhibition of lysosomal degradation}

All cells were treated $24 \mathrm{~h}$ prior to harvesting with respective cell culture medium supplemented with a final concentration of $200 \mu \mathrm{M}$ Leupeptin (dissolved in $\mathrm{dd}_{2} \mathrm{O}$ ) for a duration of $24 \mathrm{~h}$. Treatment of GBP1-silenced cells and respective control transfections was achieved by addition of a pre-diluted Leupeptin stock ( $5 \mathrm{mM}$ in growth medium) to the growth medium present in respective wells, similarly yielding a final concentration of $200 \mu \mathrm{M}$ Leupeptin. Supplementing growth medium with equal volumes of $\mathrm{ddH}_{2} \mathrm{O}$ served as treatment for the experimental control.

\subsection{Viability assays}

\subsubsection{LDH-assay}

Cytotoxicity assays were performed in a 96-well-plate under treatments listed above in serum-reduced DMEM. Supplementing growth medium with a final concentration of $1 \% \mathrm{v} / \mathrm{v}$ Triton X-100 served as internal control causing all present cells to disintegrate, therefore leading to the highest signal possible. Determination of lactate dehydrogenase (LDH) activity in cell culture media was performed using the LDH Cytotoxicity Detection Kit (Clontech, Mountain View, US) according to the manufacturer's instructions. Absorbance of LDH-dependently formed Formazan was measured with a plate-reader (Infinite M1000; TECAN, Switzerland) at a wavelength 
of $492 \mathrm{~nm}$ with a reference wavelength of $680 \mathrm{~nm}$. Percentages of intact cells referred to the experimental control were calculated according to Equation (1).

$$
\% \text { intact cells }=\frac{\left[1-\left(\frac{\left(\mathrm{AU}_{492}-\mathrm{AU}_{680}\right)_{\text {treatment }}}{\left(\mathrm{AU}_{492}-\mathrm{AU}_{680}\right)_{\text {Triton } \mathrm{X}-100}}\right)\right]}{\left[1-\left(\frac{\left(\mathrm{AU}_{492}-\mathrm{AU}_{680}\right)_{\text {control }}}{\left(\mathrm{AU}_{492}-\mathrm{AU}_{680}\right)_{\text {Triton } \mathrm{X}-100}}\right)\right]} \cdot 100
$$

$\mathrm{AU}=$ absorption units

\subsubsection{PrestoBlue assay}

Cytostaticity assays were performed in a 96-well-plate under treatments listed above. Supplementing growth medium with a final concentration of $1 \% \mathrm{v} / \mathrm{v}$ Triton X-100 served as qualitative, internal control causing all present cells to disintegrate, therefore leading to the lowest signal possible. Determination of redox-metabolic activity in living cells was performed using the PrestoBlue ${ }^{\mathrm{TM}}$ Cell Viability Reagent (ThermoScientific, Braunschweig, Germany) according to the manufacturer's instructions. Fluorescence of NADH/ $\mathrm{H}^{+}$-dependently formed Resorufin was measured with a platereader (Infinite M1000; TECAN, Switzerland) at Ex560/Em590. Percentages of redoxmetabolic activity in treated cells referred to the experimental control were calculated according to Equation (2).

$$
\% \text { metabolic activity }=\frac{\mathrm{RFU}_{\text {treatment }}}{\mathrm{RFU}_{\text {control }}} \cdot 100
$$

$\mathrm{RFU}=$ relative fluorescence units

\subsection{Luciferase reporter-assays}

Cells were seeded in 6-well-plates, transfected with either pGL3-ISRE-Luc, pGL3GAS-Luc or pGL3-GBP1-Luc and treated as described above. Cell culture supernatant was aspirated at the time of harvesting and cells were washed twice with PBS at RT. Cell lysis was achieved by addition of luciferase lysis buffer and incubation for $10 \mathrm{~min}$ on ice. Subsequently, lysates were cleared of debris by centrifugation for $10 \mathrm{~min}$ at $16,000 \mathrm{xg}$ at $4{ }^{\circ} \mathrm{C}$. Cleared cell lysate were transferred into a white microtitre plate and chemiluminescence was measured in a chemiluminometer (Orion II Microplate Luminometer; Titertek, Pforzheim, Germany) over a course of $10 \mathrm{~s}$ after the addition of firefly-luciferase substrate with the help of a built-in liquid dispenser. Normalisation of measured values was achieved by assessing relative protein concentration in lysates via a Bradford assay (Sigma Aldrich, Hamburg, Germany), which was performed according to the manufacturer's instructions. Resulting absorption at $595 \mathrm{~nm}$ was measured in a microtiter plate in a plate-reader (Infinite M1000; TECAN, Switzerland). As a blank value in Bradford assays, luciferase lysis 
buffer was used. Relative changes in chemiluminescence referred to respective experimental controls were calculated according to Equation (3).

$$
\text { Fold-change } \mathrm{RLU} / \mathrm{s}=\frac{\left(\frac{\frac{R L U}{S} \text { treatment }}{\left(\mathrm{AU}_{\text {treatment }}-\mathrm{AU}_{\text {blank }}\right)}\right)}{\left(\frac{\frac{R L U}{S} \text { control }}{\left(\mathrm{AU}_{\text {control }}-\mathrm{AU}_{\text {blank }}\right)}\right)} \cdot 100
$$

$\mathrm{RLU} / \mathrm{s}=$ relative light units per second

$\mathrm{AU}=$ absoprtion units (Bradford)

\subsection{Transcriptome profiling}

Cells were seeded in 6-well-plates and cultivated over a course of 4 days. Cell culture supernatant was aspirated at the time of harvesting and cells were washed twice with PBS at RT. Subsequently, total intracellular mRNA was isolated with the RNeasy Mini Kit (QIAGEN, Hilden, Germany) according to the manufacturer's instructions. Removal of potential DNA-contaminations in isolated RNA was achieved via an oncolumn digest with the RNase-Free DNase Set (QIAGEN, Hilden, Germany) according to the manufacturer's instructions.

Samples were sent to the Federal Institute of Drugs and Medical Devices, Bonn, Germany (Division of Pharmacogenomics; Denna Tabari, Dr. Catharina Scholl and Prof. Dr. Julia C. Stingl), where differential gene-expression between uninfected and HEV-infected cells was assessed as described elsewhere [398]. Gene-expression profiles are deposited at Gene Expression Omnibus (https://www.ncbi.nlm.nih.gov/geo/) under identifier GSE157820.

\section{8. $R T-q P C R$}

\subsubsection{Intracellular transcripts}

Cells were seeded in 6-well-plates and treated as described above. Cell culture supernatant was aspirated at the time of harvesting and cells were washed twice with PBS at RT. Cells were lysed by using peqGOLD TriFast (peqLab, Erlangen, Germany) and RNA was isolated according to the manufacturer's instructions. Air-dried RNA pellets were resuspended in $\mathrm{ddH}_{2} \mathrm{O}$ supplemented with $0.1 \%$ DEPC. Subsequently, DNA-contaminations were removed by addition of 1 Unit RQ1 RNase-free DNase (Promega $\mathrm{GmbH}$, Walldorf, Germany) and incubation for $1 \mathrm{~h}$ at $37^{\circ} \mathrm{C}$. cDNA synthesis was performed in the presence of $5 \mu \mathrm{M}$ random hexamer primers and $1 \mu \mathrm{M} \mathrm{dNTPs}$ 
using 200 Units RevertAid H Minus RT (Thermo Scientific, Braunschweig, Germany) with an RNA concentration of $0.25 \mu \mathrm{g} / \mu \mathrm{L}$ over the course of $1 \mathrm{~h}$ at $42{ }^{\circ} \mathrm{C}$. The reaction was stopped by incubation for $5 \mathrm{~min}$ at $72{ }^{\circ} \mathrm{C}$.

Analysis of gene-expression was performed with a cDNA concentration equivalent to $7.5 \mathrm{ng} / \mu \mathrm{L}$ input-RNA in the presence of $250 \mathrm{nM}$ forward and reverse primer using the Maxima SYBR-Green qPCR Kit (Thermo-Scientific, Braunschweig, Germany) according to the manufacturer's instructions with a LightCycler480 instrument (Roche Diagnostics, Mannheim, Germany). Measurement of $\mathrm{ddH}_{2} \mathrm{O}$ and non-reversetranscribed RNA served as qualitative, internal controls. Reactions were carried out in conical well, heat-sealed microtitre plates. The program was set to $10 \mathrm{~min}$ initiation at $95^{\circ} \mathrm{C}$, followed by 45 amplification cycles $\left(95^{\circ} \mathrm{C}\right.$ for $15 \mathrm{~s}, 60^{\circ} \mathrm{C}$ for $30 \mathrm{~s}$ and $72{ }^{\circ} \mathrm{C}$ for $30 \mathrm{~s}$ ) with fluorescence measurements occurring after elongation. A melting curve of amplicons starting from $60{ }^{\circ} \mathrm{C}$ to $95^{\circ} \mathrm{C}$ with continuous measurement of fluorescence served as quality control determining amplicon melting points. Determination of $\mathrm{C}_{\mathrm{q}^{-}}$ values was performed with the LightCycler480 software (Roche Diagnostics, Mannheim, Germany) using the Second Derivative Maximum method. Relative changes in gene-expression were calculated according to Equation 4.

$$
\begin{aligned}
& \text { Fold-change }=2^{-\left[\left(\mathrm{Cq}_{\text {treated }}[\mathrm{GOI}]-\mathrm{Cq}_{\text {treated }}[\mathrm{REF}]\right)-\left(\mathrm{Cq}_{\mathrm{control}}[\mathrm{GOI}]-\mathrm{Cq}_{\mathrm{control}}[\mathrm{REF}]\right)\right]} \\
& \mathrm{REF}=\text { reference gene } \\
& \mathrm{GOI}=\text { gene of interest }
\end{aligned}
$$

\subsubsection{Extracellular viral RNA}

Harvested cell culture supernatants, density-gradient fractions or infection stocks were diluted 1:5 in $\mathrm{ddH}_{2} \mathrm{O}$ supplemented with $0.1 \%$ DEPC. Prior to dilution, cell culture supernatants were rid of cell debris via centrifugation for $10 \mathrm{~min}$ at 1,000 xg at $4{ }^{\circ} \mathrm{C}$. Subsequent measurement of viral genomes was performed using the LightMix Modular Hepatitis E Virus Kit (TIB MolBiol, Berlin, Germany) in combination with the LightCycler® Multiplex RNA Virus Master (Roche Diagnostics GmbH, Mannheim, Germany) according to the manufacturer's instructions. Measurement of $\mathrm{ddH}_{2} \mathrm{O}$ and a kit internal HEV RNA standard (input being equivalent to 1,000 genomic copies) served as internal control and quantification standard, respectively. Reactions were carried out in conical well, heat-sealed microtitre plates. The concentration of HEV RNA in samples was calculated according to Equation (5) using an internal standard curve. 


$$
\text { genomes } / \mathrm{mL}=10 \frac{\mathrm{Cq}_{\mathrm{sample}}-\left(9.93+\mathrm{Cq}_{\mathrm{standard}}\right)}{-3.31} \cdot 1,000
$$

Relative changes in extracellular genomes were calculated on the basis of Equation (5) by applying Equation (6).

$$
\text { Fold-change extracellular HEV RNA }=\frac{\text { genomes } / \mathrm{mL}_{\text {treatment }}}{\text { genomes } / \mathrm{mL}_{\text {control }}}
$$

Percentages of HEV RNA present in each fraction of a density-gradient were calculated according to Equation (7).

$$
\% \mathrm{HEV} \text { genomes in fraction }=\frac{\text { genomes } / \mathrm{mL}_{\text {fraction }}}{\sum \text { genomes } / \mathrm{mL}_{\text {gradient }}} \cdot 100
$$

\subsection{Western blot analyses}

Cells were seeded in 12-well plates or 6-well-plates and treated or transfected as described above. At the time of harvesting, cell culture supernatant was aspirated and cells were washed twice with PBS at RT. Lysis was achieved by addition of RIPA lysis buffer and subsequent incubation for $10 \mathrm{~min}$ on ice. Cell lysates were sonicated for $5 \mathrm{~s}$ with a sonication probe, supplemented with SDS loading buffer and boiled for $10 \mathrm{~min}$ at $95^{\circ} \mathrm{C}$.

Electrophoretic separation occurred in a vertical electrophoresis system with $10 \%$ SDS-PAGE-gels being submerged in 1x SDS running buffer. Separated proteins were transferred onto a methanol-activated PVDF-membrane by applying a current of $1.5 \mathrm{~mA} / \mathrm{cm}^{2}$ in a semi-dry blot approach, which were subsequently blocked in $1 \times$ RotiBlock or $10 \% \mathrm{w} / \mathrm{v}$ skim milk powder dissolved in TBS-T for $30 \mathrm{~min}$ at RT. Probing of membranes with primary antibodies diluted in respective blocking buffers was performed overnight at $4{ }^{\circ} \mathrm{C}$ with subsequent washing with TBS-T being performed at RT. Membranes were further probed with fluorescently-labelled (IRDye ${ }^{\circledR}$ 680RD or IRDye® $800 \mathrm{CW}$ ) secondary antibodies diluted in TBS-T for $1 \mathrm{~h}$ at RT. Fluorescence of secondary antibodies was detected after washing with TBS-T in a LI-COR Odyssey infrared imager (LI-COR Biosciences, Lincoln, US) with wavelengths adjusted to IRDye ${ }^{\circledR}$ 680RD (Ex672/Em694) or IRDye® ${ }^{\circledR}$ 800CW (Ex774/Em789) fluorescent spectra. Images were converted from pseudo-colour to grey scale and cropped to show relevant protein bands to be presented in respective figures.

Quantification of fluorescent band-intensities was performed using the Image Studio Lite software (LI-COR Biosciences, Lincoln, US) with local background surrounding 
the regions of interest being subtracted from the selected band. Signal intensities corresponding to proteins of interest were normalised to signal intensities of reference proteins (GAPDH or $\beta$-Actin) in each sample and referred to respective experimental controls according to Equation (8).

$$
\text { Fold-change protein level }=\frac{\left(\frac{\text { Signal }_{\mathrm{POI}}}{\text { Signal }_{\mathrm{REF}}}\right)_{\text {treatment }}}{\left(\frac{\text { Signal }_{\mathrm{POI}}}{\text { Signal }_{\mathrm{REF}}}\right)_{\text {control }}}
$$

$\mathrm{POI}=$ protein of interest

$\mathrm{REF}=$ reference protein

\subsection{Immunofluorescence microscopy}

Mowiol-mounted samples on glass slides were analysed with the help of a Confocal Laser Scanning Microscope Leica TCS SP8 System with a DMi8 microscope (Leica, Wetzlar, Germany) at room temperature. A 100x magnification oil immersion objective (numerical aperture $=1.4$ ) was used and the pinhole was set to $1.3 \mathrm{AU}$, resulting confocal section thickness of $0.895 \mu \mathrm{m}$. Image acquisition and image quantification was performed using the LAS X Control Software (Leica, Wetzlar, Germany) or FIJI [399]. For quantification of protein amounts, the corrected total cell fluorescence (CTCF) was calculated according to Equation (9). A minimum of 5 cells were analysed.

$$
\mathrm{CTCF}=\operatorname{IntDen}_{P O I}-\left(A_{P O I} \cdot A B\right)
$$

IntDen = integrated signal density

$A=$ area of selection

$\mathrm{AB}=$ average signal background

$\mathrm{POI}=$ protein of interest

For assessment of co-localisation between stained components of the cell, the thresholded Mander's overlap coefficient (tMOC) was determined. A minimum of 5 cells were analysed. This coefficient is free of intensity-based bias, unlike the Pearson's coefficient, as it ignores relative differences in signals between compared channels. The tMOC assesses the grade of pixel-presence of a channel of interest within pixels being present in a region of interest in the reference channel. Values yielded by assessment of $\mathrm{tMOC}$ range from 0 (no overlapping pixels) to 1 (all pixels overlapping).

\subsubsection{Immunofluorescent stain of ethanol-fixed samples}

The following procedure was applied as standard for immunofluorescence analyses, unless Filipin III or LysoTracker ${ }^{\mathrm{TM}}$ Red DND-99 were used. Cells were seeded on glass coverslips in 12-well plates treated or transfected as described above. At the time of harvesting, cell culture supernatant was aspirated and cells were washed twice with 
PBS at RT. Fixation of cells was achieved by addition of ice-cold $96 \% \mathrm{v} / \mathrm{v}$ ethanol or a 1:1 mixture of ethanol/acetone and incubation for $30 \mathrm{~min}$ at $-20^{\circ} \mathrm{C}$. Excess ethanol was removed and cells were washed once with PBS. Blocking occurred by addition of $5 \% \mathrm{w} / \mathrm{v}$ BSA in TBS-T for $15 \mathrm{~min}$ at RT. Probing of fixed samples with primary antibodies diluted in blocking buffer was performed for $1 \mathrm{~h}$ at RT with subsequent washing with TBS-T at RT. Samples were further probed with fluorescently labelled (AlexaFluor ${ }^{\circledR} 488$, AlexaFluor ${ }^{\circledR} 546$ or AlexaFluor ${ }^{\circledR} 633$ ) secondary antibodies diluted in blocking buffer supplemented with $250 \mathrm{ng} / \mathrm{mL}$ DAPI for $1 \mathrm{~h}$ at RT. Subsequently, samples were washed with TBS-T at RT and embedded upside down in Mowiol mounting medium on glass slides.

\subsubsection{Immunofluorescent stain of formaldehyde-fixed samples}

Cells were seeded on glass coverslips in 12-well plates treated or transfected as described above. Prior to harvesting, growth medium was replaced by fresh medium supplemented with a final concentration of $50 \mathrm{nM}$ LysoTracker ${ }^{\mathrm{TM}}$ Red DND-99 (Thermo Scientific, Braunschweig, Germany) and incubated for $1 \mathrm{~h}$ at $37^{\circ} \mathrm{C}$ in an appropriate incubator. At the time of harvesting, cell culture supernatant was aspirated and cells were washed twice with PBS at RT. Fixation of cells was achieved by addition of $4 \% \mathrm{v} / \mathrm{v}$ formaldehyde in PBS and incubation for $10 \mathrm{~min}$ at RT. Excess formalin was removed and cells were washed once with PBS. Blocking occurred by addition of $5 \% \mathrm{w} / \mathrm{v}$ BSA in TBS-T for $15 \mathrm{~min}$ at RT. Probing of fixed samples with primary antibodies diluted in blocking buffer was performed for $1 \mathrm{~h}$ at RT with subsequent washing with TBS-T at RT. Samples were further probed with fluorescently labelled (AlexaFluor $\AA 488$, AlexaFluor ${ }^{\circledR} 546$ or AlexaFluor $₫ 633$ ) secondary antibodies diluted in blocking buffer for $1 \mathrm{~h}$ at RT. Subsequently, samples were washed with TBS-T at RT and embedded upside down in Mowiol mounting medium on glass slides.

\subsubsection{Immunofluorescent stain with Filipin III}

Cells were seeded on glass coverslips in 12-well plates treated or transfected as described above. At the time of harvesting, cell culture supernatant was aspirated and cells were washed twice with PBS at RT. Fixation of cells was achieved by addition of $4 \% \mathrm{v} / \mathrm{v}$ formaldehyde in PBS and incubation for $10 \mathrm{~min}$ at RT. Excess formalin was removed and cells were washed once with PBS. Formaldehyde-dependently formed Schiff-bases were quenched via addition of TBS for $5 \mathrm{~min}$ at RT. Blocking occurred by addition of $5 \% \mathrm{w} / \mathrm{v}$ BSA in TBS supplemented with $0.1 \mathrm{mg} / \mathrm{mL}$ Filipin III for $30 \mathrm{~min}$ at RT. Filipin III herein represents a fluorescent sterol-chelator extracted from the fungus Streptomyces filipinensis, yielding fluorescent stain of cholesterol and oxysterols. Probing of fixed samples with primary antibodies diluted in blocking buffer (without 
Filipin III) was performed for $1 \mathrm{~h}$ at RT with subsequent washing with TBS at RT. Samples were further probed with fluorescently labelled (AlexaFluor ${ }^{\circledR} 488$, AlexaFluor ${ }^{\circledR} 546$ or AlexaFluor ${ }^{\circledR} 633$ ) secondary antibodies diluted in blocking buffer supplemented with $0.1 \mathrm{mg} / \mathrm{mL}$ Filipin III for $1 \mathrm{~h}$ at RT. Subsequently, samples were washed with TBS at RT and embedded upside down in Mowiol mounting medium on glass slides.

\subsection{Density-gradient centrifugation}

Cells were seeded in 6-well-plates and treated as described above. At the time of harvesting, cell culture supernatant was collected and centrifuged $10 \mathrm{~min}$ at 1,000 xg at $4{ }^{\circ} \mathrm{C}$ to rid the solution of cell debris. Cleared cell culture supernatant was layered onto a discontinuous density-gradient (60-10\% w/v Iodixanol in $\mathrm{ddH}_{2} \mathrm{O}$ in steps of $10 \% \mathrm{w} / \mathrm{v}$ Iodixanol from bottom to top) and centrifuged $4 \mathrm{~h}$ at $255,000 \mathrm{xg}$ at $4{ }^{\circ} \mathrm{C}$ in a TLS-55 rotor to achieve isopycnic centrifugation. Density gradients were fractionated into 10 fractions with equal volumes from top to bottom. The number of viral genomes present in fractions was assessed via RT-qPCR. Densities of fractions were assessed using a refractometer with conversion of refractive indices into density being performed as indicated by the supplier of Iodixanol.

\subsection{End point dilution assays $\left(T C I D_{50}\right)$}

Evaluation of viral titres in cell culture supernatants was achieved by subjecting collected supernatants to an end point dilution assay (EDPA). Therefore, cells were seeded in 6-well-plates and treated or transfected as described above. At the time of harvesting, cell culture supernatant was collected and centrifuged $10 \mathrm{~min}$ at 1,000 xg at $4{ }^{\circ} \mathrm{C}$ to rid the solution of cell debris. Infectious supernatant was used to infect HEVpermissive A549/D3 cells seeded in a 96-well-plate with $2.5 \times 10^{3}$ cells/well. Infection was carried out over the course of $96 \mathrm{~h}$ in six replicates per sample in a serial dilution in 1:5 dilution-steps reaching from 1:5 to 1:78,125. Uninfected A549/D3 cells served as qualitative negative control, whereas persistently HEV-infected A549 cells served as qualitative positive control.

At the time of harvesting, cell culture supernatant was aspirated and cells were fixed by addition of ice-cold $96 \% \mathrm{v} / \mathrm{v}$ ethanol and incubation for $30 \mathrm{~min}$ at $-20{ }^{\circ} \mathrm{C}$. Excess ethanol was removed and cells were washed once with PBS. Blocking occurred by addition of $5 \% \mathrm{w} / \mathrm{v}$ BSA in TBS-T for $15 \mathrm{~min}$ at RT. Probing of fixed samples with primary antibodies targeting HEV pORF2 (rbopORF2, HCD3K129) diluted in blocking buffer was performed overnight at $4{ }^{\circ} \mathrm{C}$ with subsequent washing with TBS-T at RT. Samples were further probed with HRP-conjugated secondary antibodies (d $\alpha \mathrm{rbIgG})$ diluted in blocking buffer for $1 \mathrm{~h}$ at RT. Subsequently, samples were washed with TBS$\mathrm{T}$ at RT and HRP-dependent stain was achieved by incubation with sterile-filtered 
Carbazole stain solution for $4 \mathrm{~h}$ at RT. Infected wells were identified via presence of a red precipitate with the help of a digital microscope. Subsequent calculation of the half maximal tissue culture infection dose (TCID 50 ) was performed according to Equation (10) as described previously and expressed as relative change in TCID 50 as compared to the experimental control [400].

$$
\text { Fold-change } \operatorname{TCID}_{50}=\frac{\left[10^{\left(\frac{\# \text { infected wells }}{\# \text { replicates }}+0.5\right) \cdot \log _{10} \text { dilution }}\right]}{\left[10^{\left(\frac{\# \text { infected wells }}{\# \text { replicates }}+0.5\right) \cdot \log _{10} \text { dilution }}\right]_{\text {control }}}
$$

\subsection{Patient data analyses}

Collection of patient data during routine follow-up visits was conducted by the Charité Universitätsmedizin Berlin (Department of Nephrology and Medical Intensive Care, Dr. med. Evelyn Seelow and PD Dr. med. Mira Choi). Each patient gave written, informed consent for the data being used and analysed. The study was approved by the local institutional review board of the ethics committee of Charité Universitätsmedizin Berlin, Germany (approval number EA1/249/16).

Retrospective analysis of lipid levels and HEV titres in patient sera included 42 chronically HEV-infected patients (female, $n=33$; male, $n=9$ ) covering an age-span of 24-78 years. Data of patients were accessible for 2-37 individual follow-up visits, depending on the patient. All patients received a solid-organ transplant (renal, $n=42$; renal and co-transplant, $n=7$ ). Therefore, all patients were treated with immunosuppressants (Tacrolimus, $\mathrm{n}=35$; Cyclosporin A, n=2; Belatacept, $\mathrm{n}=3$; Azathioprin, $n=1$ ) and Mycophenolate-mofetil, with one exception. Further diagnosed illnesses in the set of patients included Diabetes mellitus $(n=10)$ and different chronic hepatic infections (hepatitis B or C, n=3). An HEV-infection was assessed via testing of IgG/IgM-reactivity against HEV pORF2 or via PCR. Viral titres were quantified using qPCR and serum-parameters were assessed via standard laboratory procedures. If patients received antiviral treatment (Ribavirin or Sofosbuvir), values originating from corresponding dates were excluded from the analysis. For analysis of statin-induced changes in HEV titres, patients were grouped based on whether or not they received respective treatments at the time point of acquiring sera. Statin-treatment included the use of Simvastatin, Pravastatin, Fluvastatin or Atorvastatin. For analyses of blood-lipid kinetics upon $\mathrm{HEV}$-infection, dates and respective values of each specific patient were picked corresponding to the last follow-up visit before infection ( $t-1)$, at first positive testing $(\mathrm{t} 0)$ or the first follow-up visit after infection ( $\mathrm{t} 1)$. Outliers were identified using the ROUT method $(\mathrm{Q}=1 \%)$. 


\subsection{Statistical analyses}

Cell culture-based data of this study is displayed as value and corresponding standard error of the mean. Unless stated otherwise, an unpaired t-test was applied as a standard test for statistical significance. For multiple comparisons, a Holm-Sidak correction was applied. For datasets being presented as fold-change, values were referred to a suitable, experimental control as indicated above. Error bars for these controls cannot be displayed, as control groups were arbitrarily set as 1 , which represents standardisation performed for each of the independent assays. Independent measurements are indicated by a number $n=x$. Unless stated otherwise, experiments are based on biological replicates with $n=3$. All statistical analyses were performed using GraphPad Prism version 8.0. 


\section{Results}

\subsection{GBP1 induction and antiviral effect are most efficiently exerted by IFN $\gamma$}

PEGylated IFNs are commonly used as antiviral treatment against an HEV-infection, yet underlying mechanisms are poorly understood. Among the potentially relevant IFN-effectors is the GTPase GBP1, as it is one of the most abundantly expressed proteins in IFN-mediated innate immunity. Therefore, an initial screening of different IFNs was set up to elucidate differences in GBP1-induction in vitro. Luciferase reporter assays and qPCR were of use to investigate IFN $\alpha$-, IFN $\beta$ - or IFN $\gamma$-dependent geneexpression and antiviral effects in uninfected A549/D3 cells or persistently HEVinfected A549 cells (Figure 20).

A

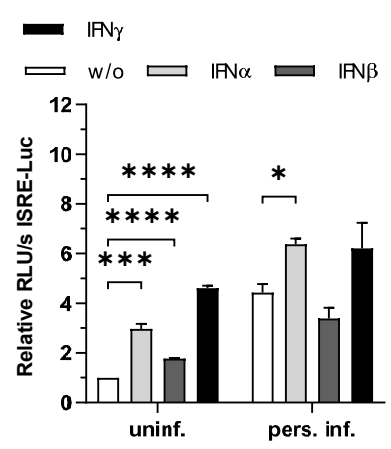

D

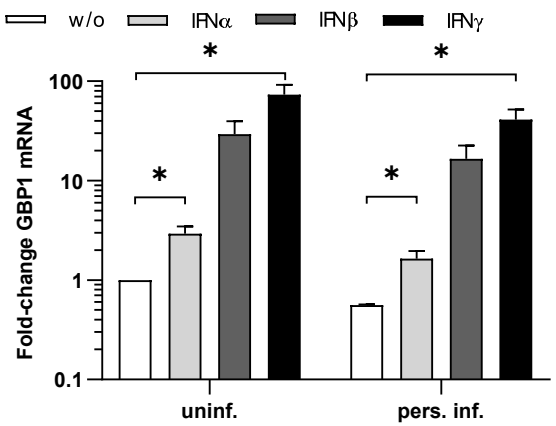

B

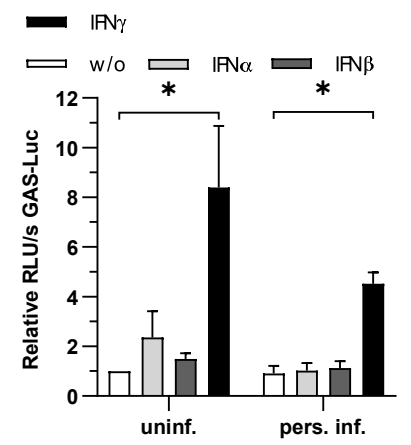

C

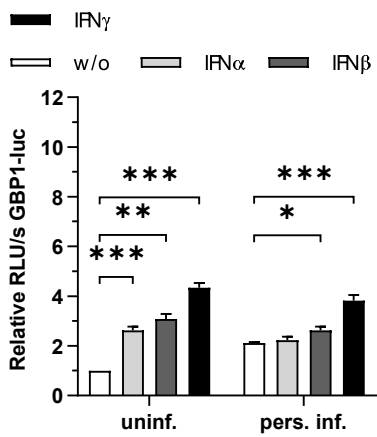

E

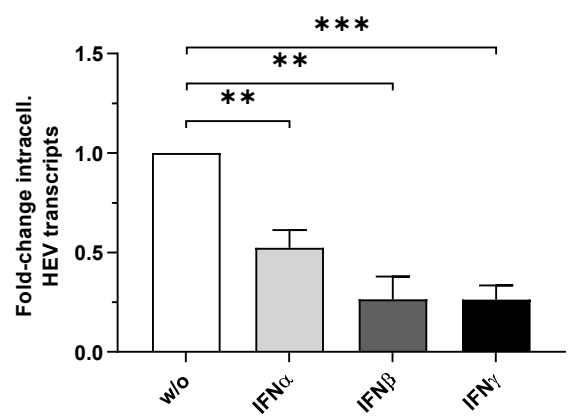

Figure 20. IFN $\gamma$ induces GBP1 promoter and gene-expression most efficiently leading to strongest antiviral effect. (A-C) Relative change in ISRE-, GAS, or proximal GBP1-promoter-driven luciferase activity upon treatment with $100 \mathrm{U} / \mathrm{mL}$ IFN $\alpha / \beta / \gamma$ over $24 \mathrm{~h}$ in uninfected or infected cells; values referred to uninfected, untreated control. (D) Relative change in intracellular GBP1 mRNA in uninfected or infected cells upon treatment with $100 \mathrm{U} / \mathrm{mL}$ IFN $\alpha / \beta / \gamma$ over $24 \mathrm{~h}$ as assessed via RT-qPCR; values referred to uninfected, untreated cells. (E) Relative change in intracellular HEV transcripts in infected cells upon treatment with $100 \mathrm{U} / \mathrm{mL}$ IFN $\alpha / \beta / \gamma$ over $24 \mathrm{~h}$ as assessed via RT-qPCR; values referred to untreated cells. uninf. = uninfected A549/D3; inf. = persistently HEVinfected A549; w/o $=$ no treatment. ${ }^{*} \mathrm{p}<0.05,{ }^{* *} \mathrm{p}<0.01,{ }^{* * *} \mathrm{p}<0.001,{ }^{* * * *} \mathrm{p}<0.0001$, unpaired $\mathrm{t}$-test with Holm-Sidak correction.

Luciferase reporter assays clearly demonstrated that all tested IFNs generally induced ISRE in uninfected cells. In infected cells, however, IFN $\beta$ failed to do so (Figure 20A). 
On the other hand, IFN $\gamma$ represented the only IFN being capable of inducing GASrelated luciferase activity (Figure 20B). These changes were partially retained for the ISRE- and GAS-containing GBP1-promoter, which was inducible by all tested IFNs in uninfected cells. IFN $\beta$ prompted a moderate induction of the promoter in persistently HEV-infected cells, yet IFN $\gamma$ herein evoked predominant changes (Figure 20C). In general, IFN $\gamma$ induced promoter-activity most efficiently. This was in line with qPCR analyses where IFN $\gamma$ elicited the strongest increase in GBP1 mRNA (Figure 20D). All tested IFNs displayed antiviral activity against HEV, as all led to a reduction in intracellular HEV transcripts. Again, this effect was most pronounced upon addition of IFN $\gamma$ (Figure 20E).

These data indicate GBP1 to be induced most efficiently by IFN $\gamma$ in both uninfected and infected cells. This directly correlates with antiviral activity being exerted predominantly by the type II IFN.

\subsection{GBP1 is induced in an early infection with HEV}

Previous experiments point towards an involvement of GBP1 in innate immunity against HEV. To evaluate an early immune-response with regards to GBP1, HEVsusceptible A549/D3 cells were infected with 10 genome equivalents (GE) of HEV virus stock (Hepeviridae, Orthohepevirus A, genotype 3c, isolate 47832c) and monitored over the course of 12 days. Within this system, HEV-mediated GBP1-inducibility was assessed on gene-expression level via qPCR and protein level via Western blot analyses or assessment of the corrected total cell fluorescence (CTCF) in confocal laser scanning microscopy (CLSM) analyses (Figure 21). 
A

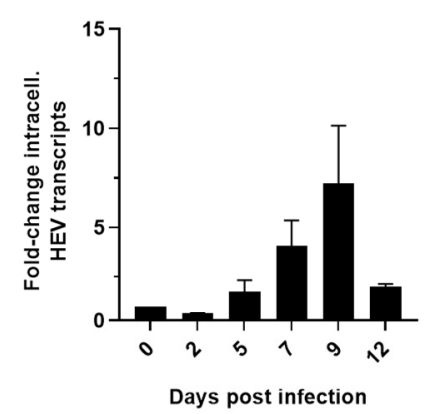

D

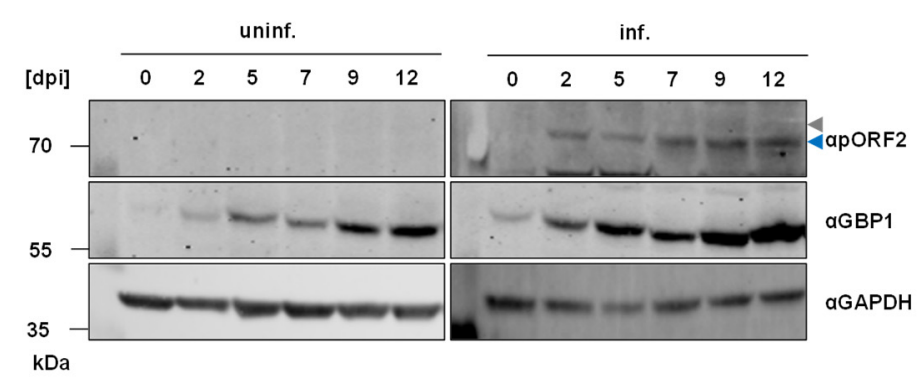

$\mathbf{F}$

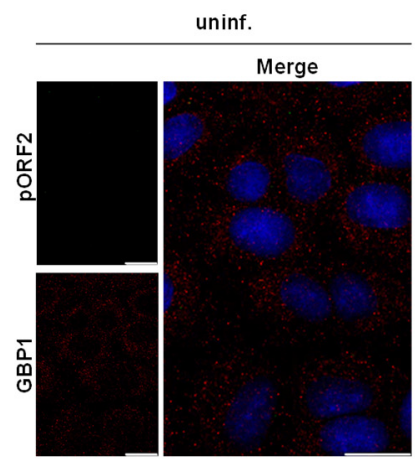

B

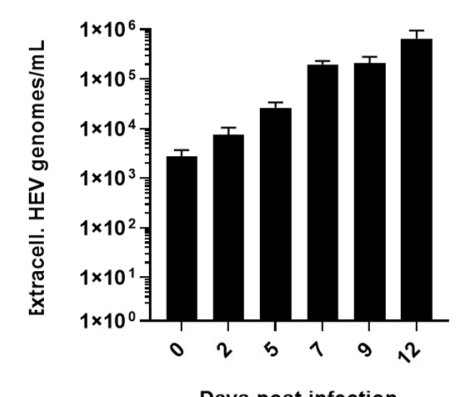

C

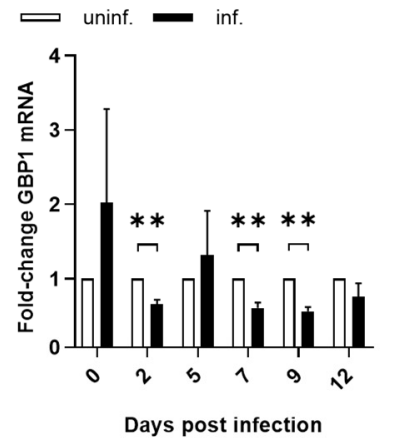

E

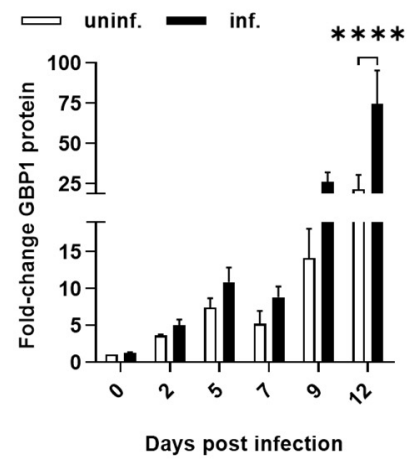

G

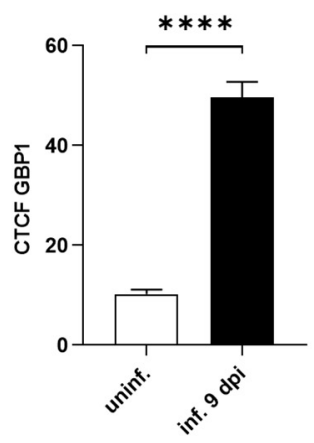

Figure 21. GBP1 mRNA and protein amount is modulated upon early HEV-infection of A549/D3 cells. (A) Relative change in intracellular HEV transcripts in infected A549/D3 cells as assessed via RT-qPCR over the course of 12 days post infection; values referred to day 0 post infection. (B) Absolute number of HEV transcripts present in cell culture supernatant of infected A549/D3 cells as assessed via RT-qPCR over the course of 12 days post infection. (C) Relative change in intracellular GBP1 mRNA in uninfected or infected A549/D3 cells as assessed via RT-qPCR over the course of 12 days post infection; values referred to respective time points of uninfected A549/D3 cells. (D) Representative Western blot of HEV pORF2, GBP1 and GAPDH in total lysates of uninfected or infected A549/D3 cells over the course of 12 days post infection; blue arrow indicates unglycosylated HEV pORF2; grey arrow indicates glycosylated HEV pORF2. (E) Relative change in GBP1 signal intensity in (D); values referred to day 0 of uninfected A549/D3 cells. (F) Representative CLSM images of DAPI (blue), HEV pORF2 (green) and GBP1 (red) of uninfected or infected A549/D3 cells at 9 days post infection; scale bar $=20 \mu \mathrm{m}$. (G) Quantification of GBP1 signal intensity in (F) expressed as CTCF. uninf. $=$ uninfected A549/D3; inf. = infected A549/D3; dpi = days post infection. ${ }^{* *} \mathrm{p}<0.01,{ }^{* * * *} \mathrm{p}<0.0001$, unpaired t-test with Holm-Sidak correction.

Successful establishment of a viral infection prevailed as early as 2-5 days post infection, as visible in quantification of both intra- and extracellular HEV RNA (Figure 21A-B). Rising amounts of HEV pORF2 further indicated an ongoing viral spread within the culture (Figure 21D). Although an initial spike in GBP1 transcripts could be observed at day 0 post infection, the amount of GBP1 mRNA generally declined in 
infected cells (Figure 21C). This contrasted with the amount of GBP1 protein, which significantly increased over the course of infection as compared to uninfected cells (Figure 21D-E). This was further evidenced by applying single-cell CLSM analyses on cells. Here, significant elevation of the GBP1 amount was found to be present as early as 9 days post infection (Figure 21F-G).

To exclude possibilities of components present in viral stocks triggering the GBP1induction, A549/D3 cells were infected with both an active HEV inoculate as well as with a UV-inactivated inoculate. Protein amount of GBP1 was subsequently assessed via Western blot analyses at 12 days post infection (Figure 22).

A

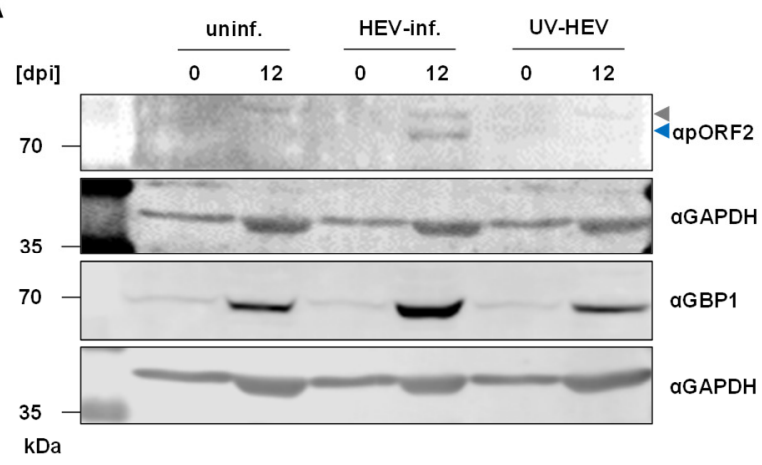

B

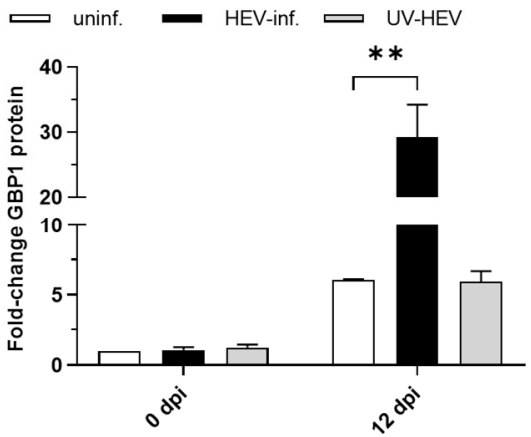

Figure 22. Induction of GBP1 is due to successful HEV-infection. (A) Representative Western blot of HEV pORF2, GBP1 and GAPDH in total lysates of uninfected, HEV-infected or UV-HEV subjected A549/D3 cells at 0 or 12 days post infection; blue arrow indicates unglycosylated HEV pORF2; grey arrow indicates glycosylated HEV pORF2. (B) Relative change in GBP1 signal intensity in (A); values referred to day 0 of uninfected A549/D3 cells. uninf. = uninfected A549/D3; HEV-inf. = infected A549/D3; UV-HEV = cells subjected to UV-treated viral stock; dpi = days post infection. ${ }^{* *} \mathrm{p}<0.01$, unpaired t-test with Holm-Sidak correction.

An HEV-infection only established in cells infected with the active viral stock, as evidenced by the presence of the viral capsid protein in Western blot analyses (Figure 22A). No HEV pORF2 could be observed in cells infected with UV-inactivated HEV preparations. Concordantly, GBP1 protein levels only increased in cells displaying an established infection (Figure 22A-B).

Summarising these findings indicates that an HEV-infection reduces GBP1-coding mRNA. However, the opposite is the case for the GBP1 protein amount, which is significantly elevated after 9-12 days post infection.

\subsection{GBP1 is induced in a stable, persistent infection with HEV}

Effects caused by an early infection with HEV may differ from a stable, persistent viral infection with respect to ongoing cellular processes. Thus, HEV-mediated GBP1inducibility was assessed in the standard cell culture system used throughout this study, namely persistently HEV-infected A549 cells. These were analysed with respect 
to GBP1 promoter-activity, gene-expression, and protein amount as compared to uninfected A549/D3 cells after 4 days of culturing (Figure 23).

A

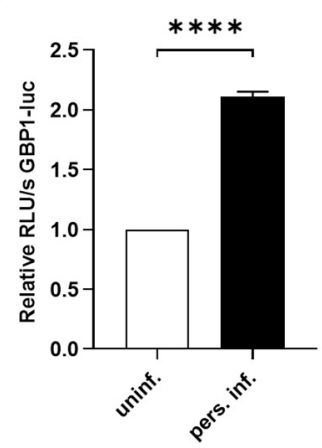

E

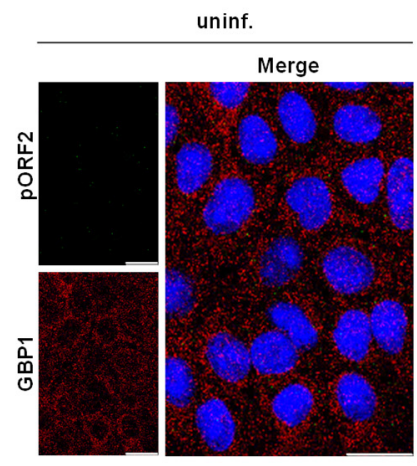

B

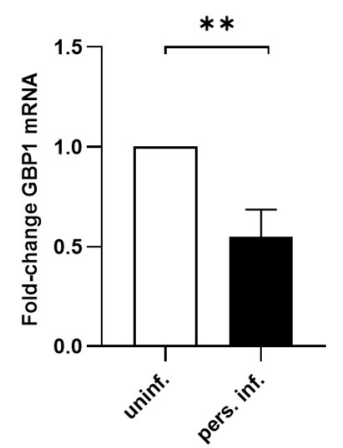

C

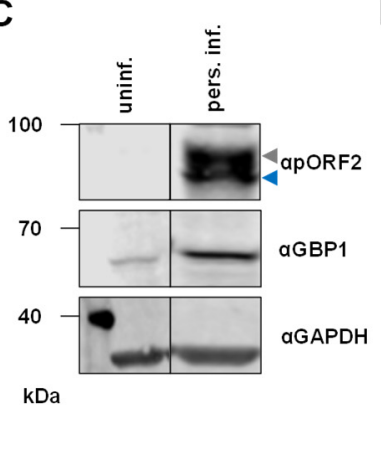

D

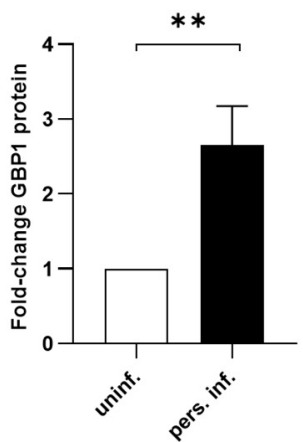

$\mathbf{F}$ pers. inf.

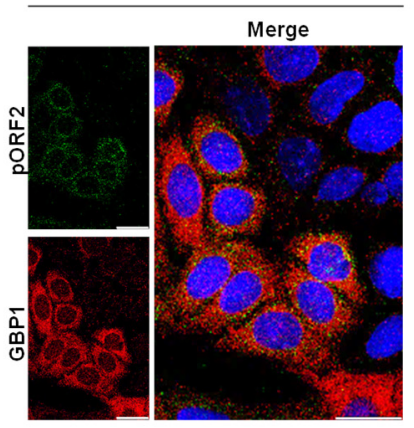

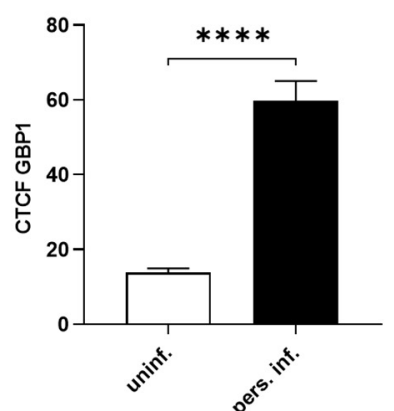

Figure 23. GBP1 promoter activity, mRNA and protein amount is modulated in persistently HEV-infected A549 cells. (A) Relative change in proximal GBP1-promoter driven luciferase activity in uninfected or infected cells; values referred to uninfected cells. (B) Relative change in intracellular GBP1 mRNA in uninfected or infected cells as assessed via RT-qPCR; values referred to uninfected cells. (C) Representative Western blot of HEV pORF2, GBP1 and GAPDH in total lysates of uninfected or infected cells; blue arrow indicates unglycosylated HEV pORF2; grey arrow indicates glycosylated HEV pORF2. (D) Relative change in GBP1 signal intensity in (C); values referred to uninfected cells. (E) Representative CLSM images of DAPI (blue), HEV pORF2 (green) and GBP1 (red) of uninfected or infected cells; scale bar $=20 \mu \mathrm{m}$. (F) Quantification of GBP1 signal intensity in (E) expressed as CTCF. uninf. = uninfected A549/D3; inf. = persistently HEV-infected A549. ${ }^{* *} \mathrm{p}<0.01$, ${ }^{* * *} \mathrm{p}<0.0001$, unpaired t-test with HolmSidak correction.

As observed before, the proximal GBP1-promoter activity was induced in infected cells (Figure 23A). However, this was not reflected by an induction of GBP1 mRNA amount. In fact, infected cells displayed a significantly reduced GBP1 mRNA amount (Figure 23B). An even more complex picture was drawn by the analysis of GBP1 protein levels in infected cells. Here, GBP1 was found to be significantly induced by an infection with HEV in Western blot analyses (Figure 23C-D), which was validated via single-cell CLSM analyses under similar conditions (Figure 23E-F).

Taken together, HEV moderately induces GBP1 promoter-activity. Surprisingly, this is not reflected by an elevated amount of GBP1 mRNA, which suggests viral immuneevasion mechanisms being in place. Nonetheless, HEV elicits a rise in GBP1 protein levels, which certainly are required for GBP1-driven cellular effects. 


\subsection{GBP1 half-life is prolonged in HEV-infected cells}

An infection with HEV caused GBP1 mRNA to be reduced, yet protein levels to be increased in A549 cells. This difference is of central interest, as the protein itself is needed to exert effector functions, thus requiring elucidation. Therefore, the protein half-life of GBP1 was assessed in uninfected and HEV-infected cells in a Cycloheximide (CHX) chase-assay and subsequent Western blot analyses (Figure 24).

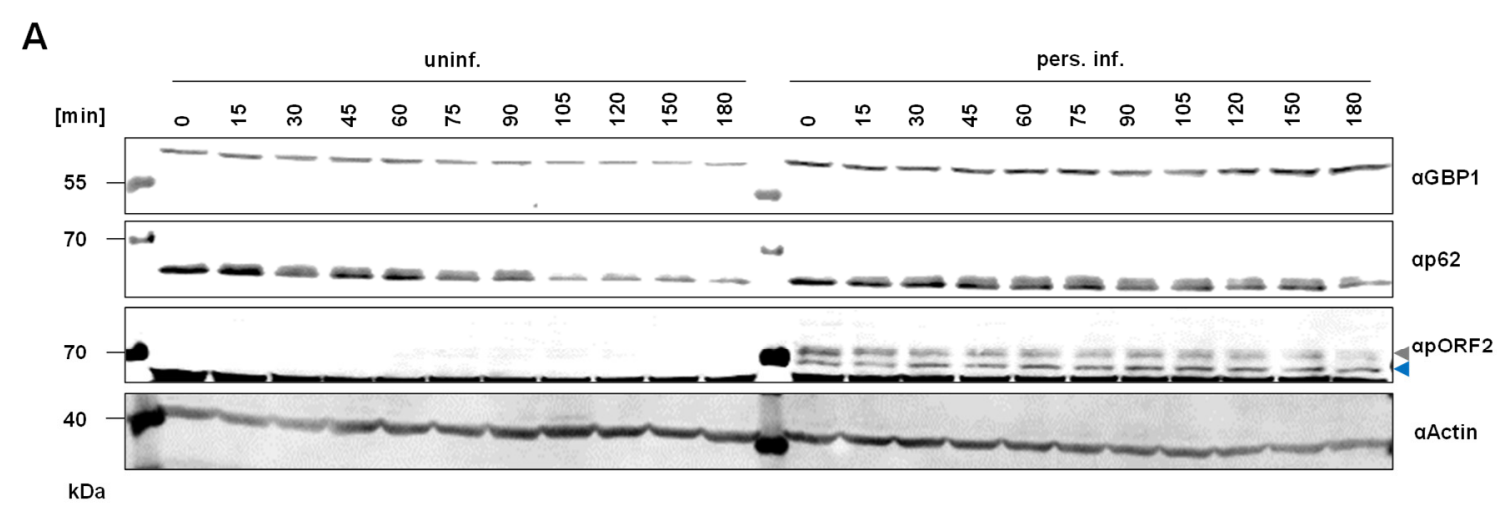

B

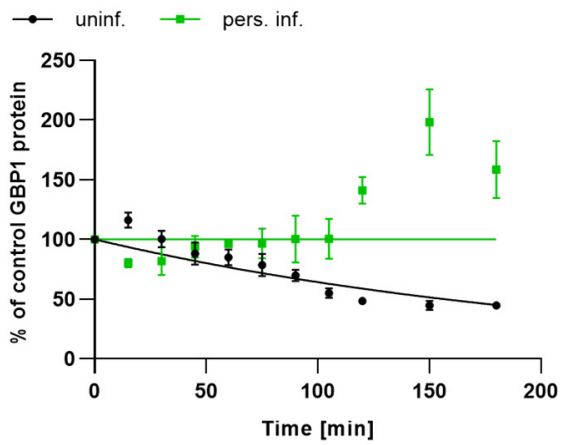

C

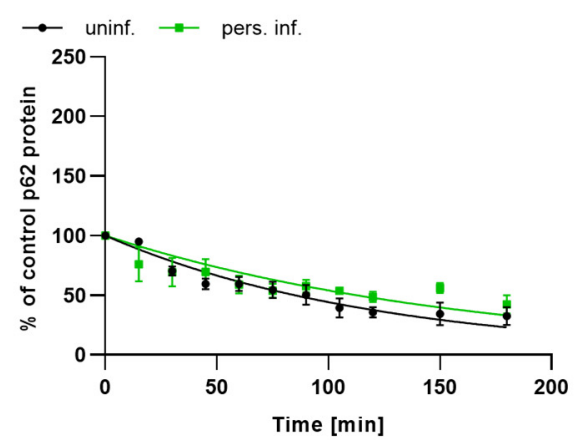

Figure 24. Stabilising effect on GBP1 half-life exerted by an HEV-infection in A549 cells. (A) Representative Western blot of HEV pORF2, GBP1, p62 and $\beta$ Actin in total lysates of uninfected or infected cells over the course of 0 to $180 \mathrm{~min} 100 \mu \mathrm{g} / \mathrm{mL}$ CHX-treatment; blue arrow indicates unglycosylated HEV pORF2; grey arrow indicates glycosylated HEV pORF2. (B) Relative change in GBP1 signal intensity in (A); values expressed as \% of signal intensity at 0 min CHX-treatment in respective cell line; curve fitting applied as one-phase decay model with intercept set to 100 and decay set to reach $0 \%$. (C) Relative change in p62 signal intensity in (A); values expressed as \% of signal intensity at 0 min $\mathrm{CHX}$-treatment in respective cell line; curve fitting applied as one-phase decay model with intercept set to 100 and decay set to reach $0 \%$. uninf. = uninfected A549/D3; inf. = persistently HEVinfected A549.

The CHX chase-assay was controlled by assessing the protein half-life of the shortlived protein p62. Both in uninfected and infected cells, p62 levels remitted over time displaying a half-life of $86 \pm 11 \mathrm{~min}\left(\mathrm{R}^{2}=0.81\right)$ or $116 \pm 23 \mathrm{~min}\left(\mathrm{R}^{2}=0.39\right)$ within a $95 \%$ confidence interval, respectively (Figure 24A and C). Given a properly working translation-inhibition, as evidenced by decreasing p62 levels, GBP1 protein half-life was found to be in a range of $160.5 \pm 29.5 \mathrm{~min}\left(\mathrm{R}^{2}=0.74\right)$ in uninfected cells within a $95 \%$ confidence interval. Interestingly, GBP1 protein levels failed to decrease in HEVinfected cells, indicating a strongly stabilising effect of the protein, although 
mathematical determination of the protein half-life was not possible (Figure 24A and B).

Although GBP1 mRNA is significantly reduced upon HEV-infection, protein levels are induced due to a stabilising effect exerted on GBP1.

\subsection{Overexpression of GBP1 restricts HEV via lysosomal degradation}

The type II interferon IFN $\gamma$ induces GBP1 most efficiently in A549 cells, when compared to IFN $\alpha$ or IFN $\beta$. Similarly, IFN $\gamma$ exerts the strongest antiviral effect against HEV. To test for a causal relationship, GBP1 was overexpressed in uninfected and infected cells via transfection of a plasmid encoding the N-terminally FLAG-tagged GBP1 wild-type protein (GBP1wt). Resulting antiviral activities were analysed using Western blot analyses in the presence or absence of IFN $\gamma$. A virus titration via an end point dilution assay (EPDA) helped to gain insights into viral release (Figure 25).

A

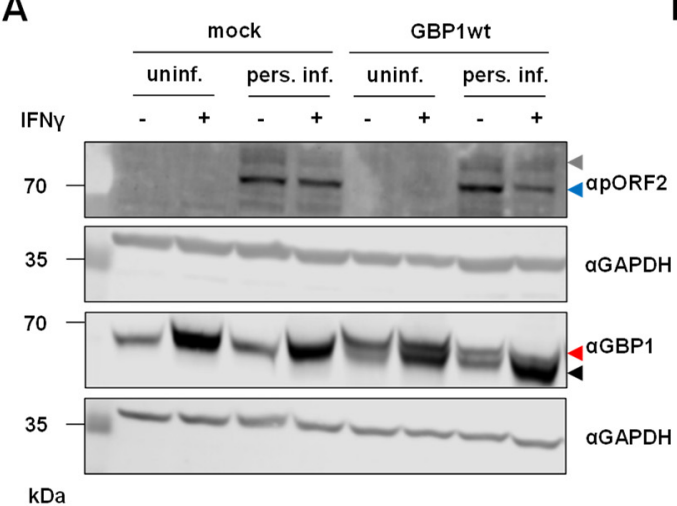

B

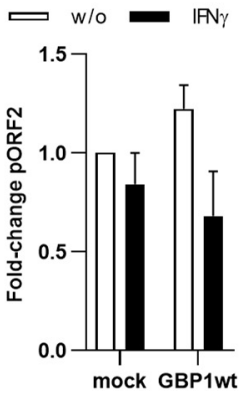

C

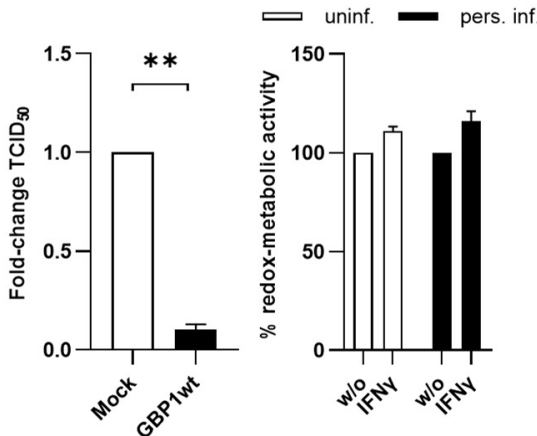

Figure 25. Overexpression of wild-type GBP1 reduces viral release from persistently HEV-infected A549 cells. (A) Representative Western blot of HEV pORF2, GBP1 and GAPDH in total lysates of mock transfected or pCMV2btag-GBP1-wt transfected uninfected or infected cells without and with $100 \mathrm{U} / \mathrm{mL}$ IFN $\gamma$ over 24 h; blue arrow indicates unglycosylated HEV pORF2; grey arrow indicates glycosylated HEV pORF2; black arrow indicates endogenous GBP1; red arrow indicates FLAG-tagged GBP1. (B) Relative change in HEV pORF2 signal intensity in (A); values referred to infected, mock transfected, untreated cells. (C) Relative change in number of infectious viral particles being released into cell culture supernatant of transfected and infected cells as assessed in an EPDA; values referred to mock transfected, infected cells. (D) Cell viability of uninfected and infected cells upon $24 \mathrm{~h}$ treatment with $100 \mathrm{U} / \mathrm{mL}$ IFN $\gamma$ as assessed via a PrestoBlue assay; values expressed as \% metabolic activity referred to the experimental control. uninf. $=$ uninfected A549/D3; inf. $=$ persistently HEV-infected A549; w/o $=$ no treatment. ${ }^{* *}$ $\mathrm{p}<0.01$, unpaired t-test with Holm-Sidak correction.

An overexpression of GBP1wt did not lead to a significant reduction in intracellular HEV pORF2 in Western blot analyses. Solely by the addition of IFN $\gamma$ a reductive trend was unveiled (Figure 25A-B) without disturbing cell viability (Figure 25D). Despite this, elevated GBP1wt levels did evoke a major drop in the number of released infectious viral particles (Figure 25C).

To rid the experimental system from transfection-efficiency-based distortions in Western blot analyses, the intracellular amount of HEV pORF2 was analysed via 
single-cell CLSM analyses quantifying protein levels in GBP1wt-overexpressing cells only. Further, subcellular localisation of pORF2 was monitored with respect to the lysosomal marker protein LAMP2. Leupeptin, a cysteine-, serine- and threonineprotease inhibitor, was applied to reduce lysosomal degradation. This allowed to pinpoint whether observed effects are related to lysosomal degradation of HEV (Figure 26).

A
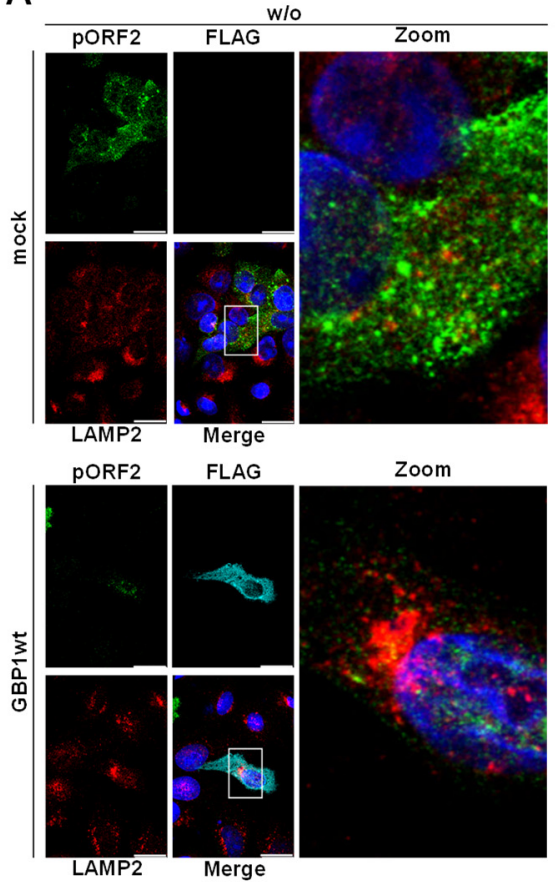

D

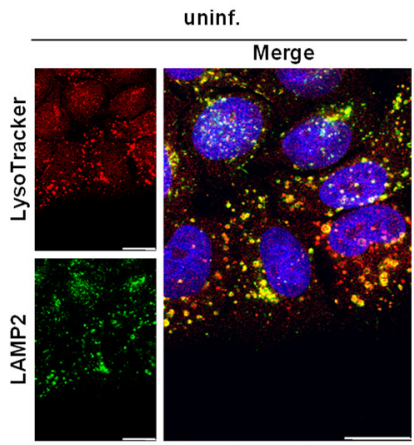

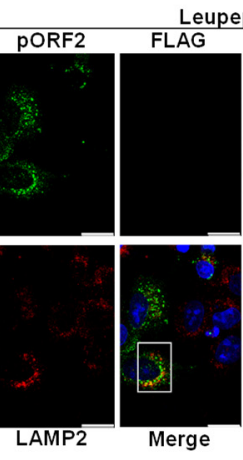

eupeptin

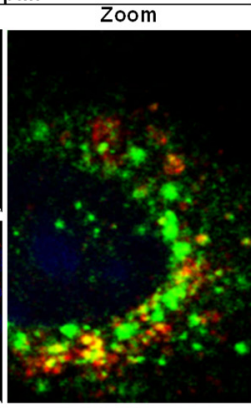

PORF2

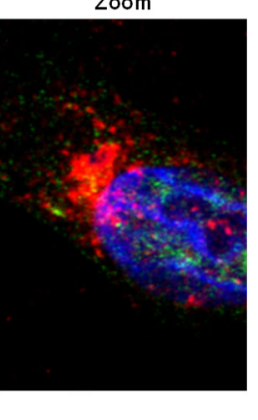

$E$
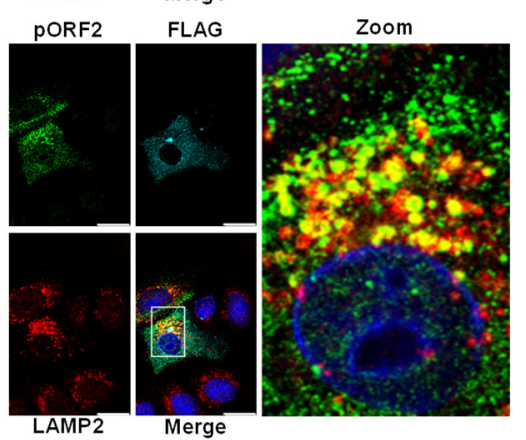

$\mathbf{F}$
B

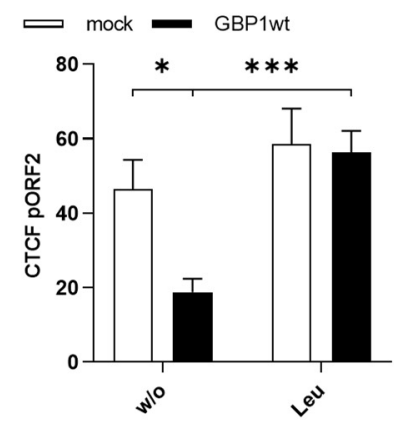

C

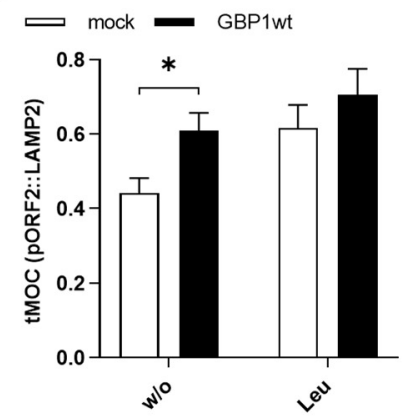

Figure 26. GBP1 overexpression induces lysosomal localisation of HEV and HEV pORF2 reduction rescuable via Leupeptin treatment. (A) Representative CLSM images of DAPI (blue), HEV pORF2 (green), LAMP2 (red) and FLAG-tagged GBP1 (cyan) of transfected, persistently HEV-infected A549 cells without or with $200 \mu \mathrm{M}$ Leupeptin treatment over $24 \mathrm{~h}$; scale bar $=20 \mu \mathrm{m}$; zoom represents magnification from area in white rectangle. (B) Quantification of HEV pORF2 signal intensity in (A) expressed as CTCF. (C) Quantification of pixel co-localisation between HEV pORF2 and LAMP2 in (A) expressed as tMOC. (D) Representative CLSM images of DAPI (blue), LAMP2 (green) and LysoTracker (red) of uninfected A549/D3 cells; scale bar $=20 \mu \mathrm{m}$. (E) Quantification of pixel co-localisation between LAMP2 and LysoTracker in (D) expressed as tMOC. (F) Cell viability of uninfected and infected cells upon $24 \mathrm{~h}$ treatment with $200 \mu \mathrm{M}$ Leupeptin as assessed via a PrestoBlue assay; values expressed as $\%$ metabolic activity referred to the experimental control. uninf. = uninfected A549/D3; inf. = persistently HEVinfected A549; $w / 0=$ no treatment; Leu = Leupeptin-treatment. ${ }^{*} \mathrm{p}<0.05,{ }^{* * *} \mathrm{p}<0.001$, unpaired t-test with HolmSidak correction. 
On a single-cell basis it became evident that overexpression of GBP1wt indeed led to a significant reduction in intracellular HEV pORF2. Importantly, this subsided under application of Leupeptin (Figure 26A-B). Herein, no loss of cell viability could be observed (Figure 26F). In concordance, co-localisation of HEV pORF2 with the lysosomal marker LAMP2, expressed as thresholded Mender's overlap coefficient (tMOC), significantly increased in GBP1wt-overexpressing cells (Figure 26A and C). Signal patterns of LAMP2 were considered to reflect actual lysosomes, as most of the resulting signals overlaid with signals yielded from LysoTracker stain, a lysosomespecific fluorophore (Figure 26D-E).

Based on these results, an overexpression of GBP1wt employs reductions in HEV pORF2 levels. Causative is the lysosomal incorporation and subsequent degradation. Consequently, the number of released HEV virions declines.

\subsection{GBP1 homodimerisation is required for lysosomal degradation of HEV}

GBP1 is a GTPase and is classified within the dynamin superfamily. Hence, several molecular aspects may be involved in triggering an antiviral effect. Two of these are either GTPase-activity, contributing to dynamin-functionality, or homodimerisation, affecting membrane-localisation. These functions can be abrogated via introduction of a point mutation in the protein sequence. Loss of GTPase functionality is achieved by mutation R48A, whereas loss of homodimerisation-capability is achieved by mutation S73A. To improve the understanding about the mode of action of GBP1, both mutants were overexpressed in infected cells and before-seen effects on HEV were re-assessed via CLSM analyses and virus titration (Figure 27). 
A

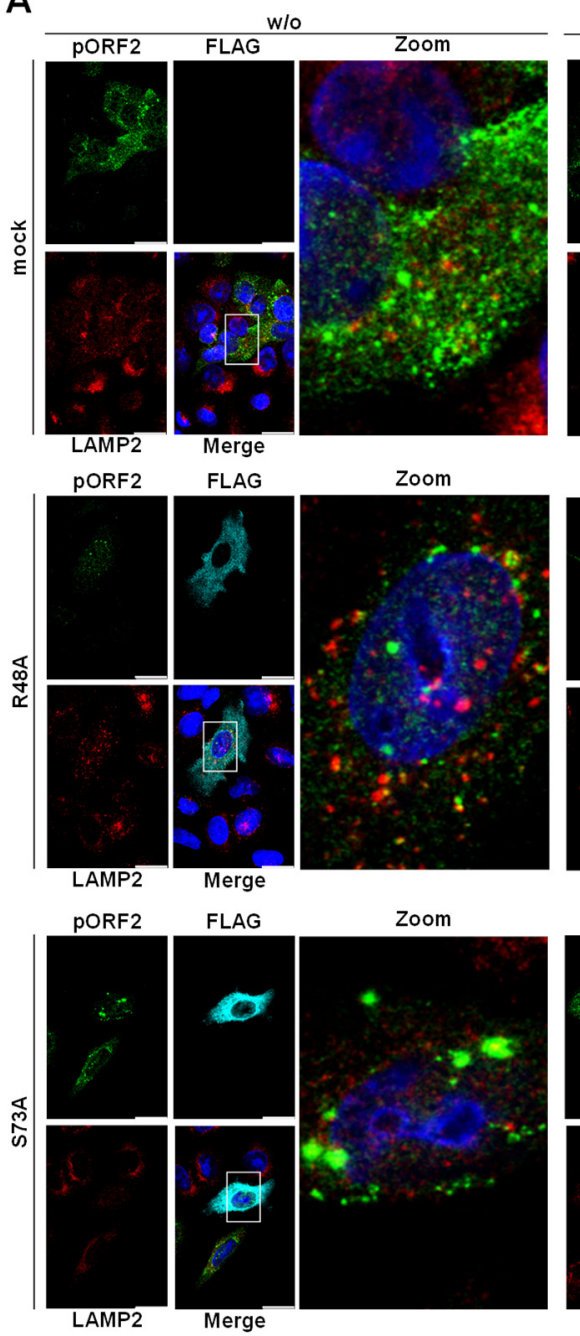

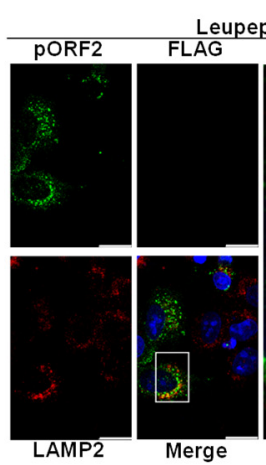
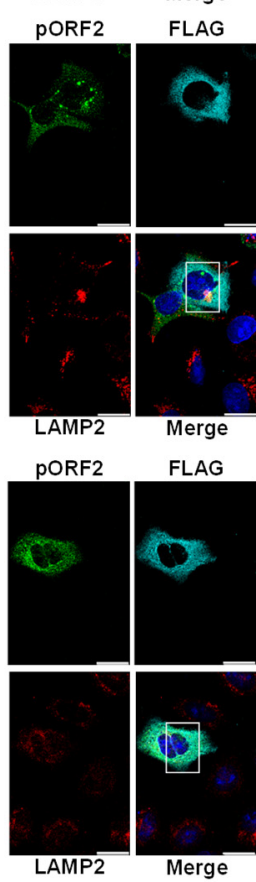

B

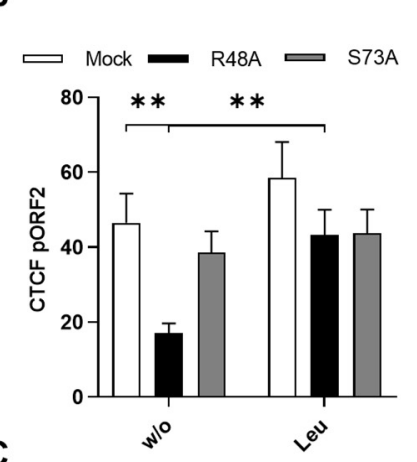

C
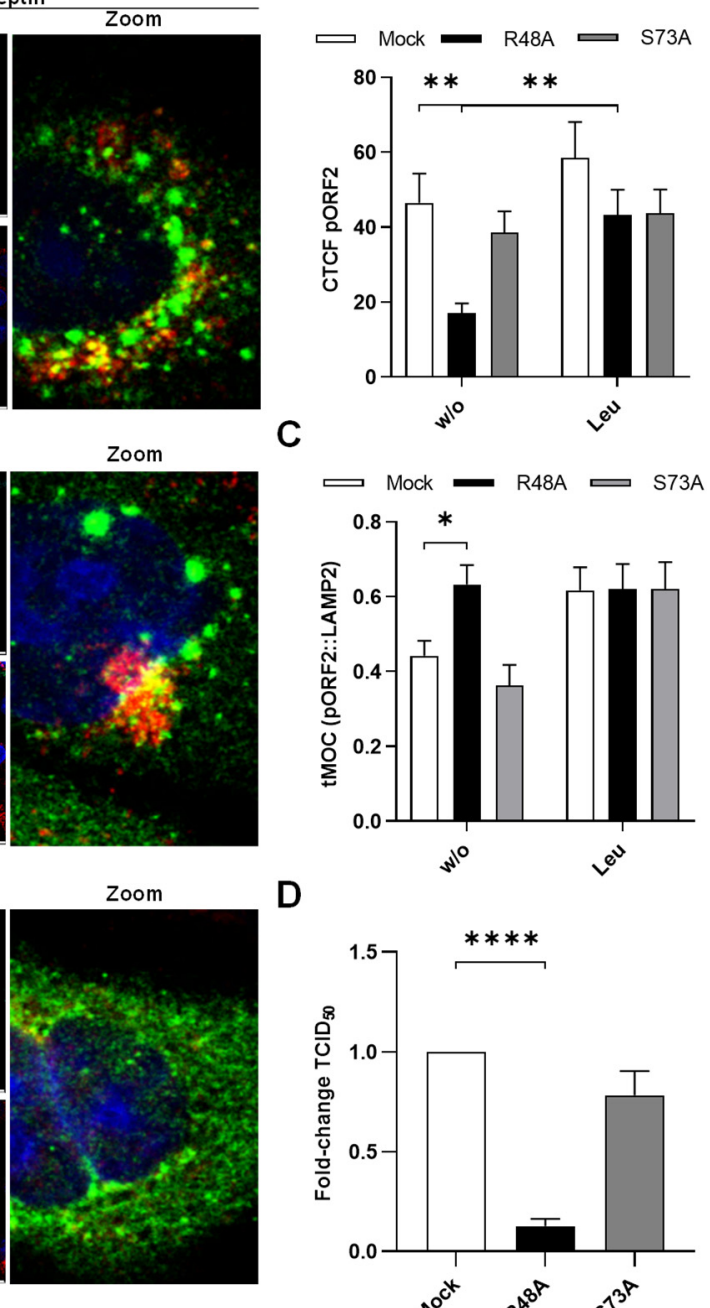

D

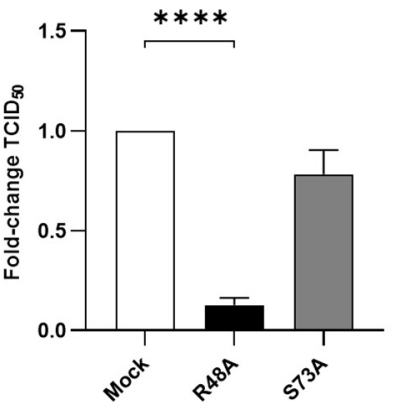

Figure 27. GBP1-S73A mutant fails to induce lysosomal HEV degradation while GBP1-R48A retains antiviral activity. (A) Representative CLSM images of DAPI (blue), HEV pORF2 (green), LAMP2 (red) and FLAG-tagged GBP1 (cyan) of transfected, persistently HEV-infected A549 cells without or with $200 \mu \mathrm{M}$ Leupeptin treatment over $24 \mathrm{~h}$; scale bar $=20 \mu \mathrm{m}$; zoom represents magnification from area in white rectangle. (B) Quantification of HEV pORF2 signal intensity in (A) expressed as CTCF. (C) Quantification of pixel co-localisation between HEV pORF2 and LAMP2 in (A) expressed as tMOC. (D) Relative change in number of infectious viral particles being released into cell culture supernatant of transfected and infected cells as assessed in an EPDA; values referred to mock transfected, infected cells. $w / o=$ no treatment; Leu $=$ Leupeptin-treatment. ${ }^{*} p<0.05,{ }^{* *} p<0.01,{ }^{* * * *} p<0.0001$, unpaired t-test with Holm-Sidak correction.

Overexpression of the GTPase-deficient mutant GBP1-R48A led to a significant reduction of intracellular HEV pORF2 amount (Figure 27A and B). This went along with a dot-like distribution and an increase in lysosomal co-localisation of the latter (Figure 27A and C). Finally, HEV virion release plummeted, once the GTPase-deficient mutant GBP1-R48A was overexpressed (Figure 27D). As for homodimerisationdeficient GBP1 harbouring an S73A mutation (GBP1-S73A), no such effects were exhibited (Figure 27A-C). Similarly, virion release remained unaffected in homodimerisation-deficient GBP1-S73A-overexpressing cells (Figure 27D). 
In conclusion, GBP1 retains antiviral effects on HEV, even if no GTP-hydrolysis can occur. An impairment of GBP1 homodimerisation however, is detrimental for antiviral activity. Thus, the restrictive effect of GBP1 on HEV is homodimerisation-dependent.

\subsection{Silencing of GBP1 rescues HEV from IFN $\gamma$-mediated lysosomal degradation}

GBP1 evidently represents a major restriction factor for $\mathrm{HEV}$ by inducing homodimerisation-dependent, lysosomal degradation of the virus. However, it also only represents one of many factors being induced by IFN $\gamma$. It therefore is of interest, whether GBP1 also represents an essential factor within the IFN $\gamma$-driven antiviral response against the Hepevirus. To address this question, uninfected and infected cells were subjected to an siRNA-based GBP1 silencing under stimulation with the type II IFN. Antiviral activity herein was monitored via quantification of intracellular HEV pORF2 levels and assessment of release of HEV virions via Western blot analyses and EPDA, respectively (Figure 28).

A

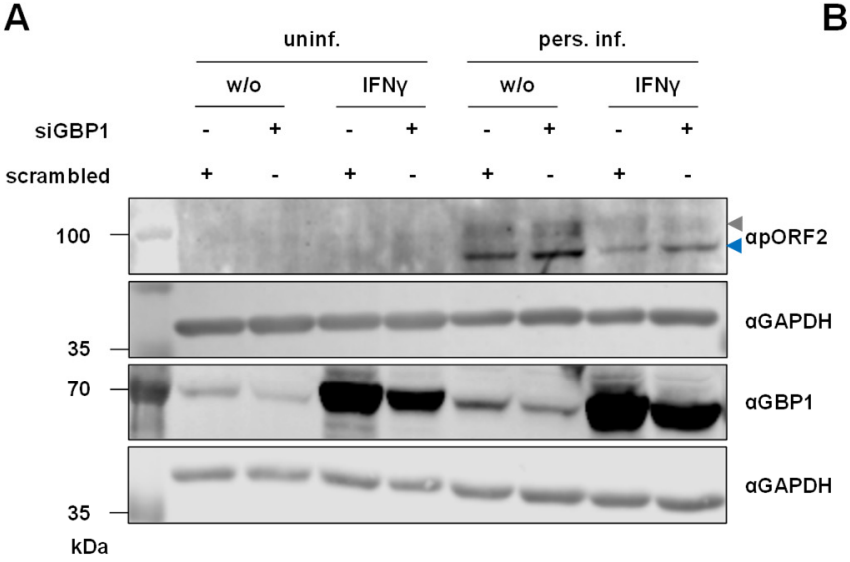

B

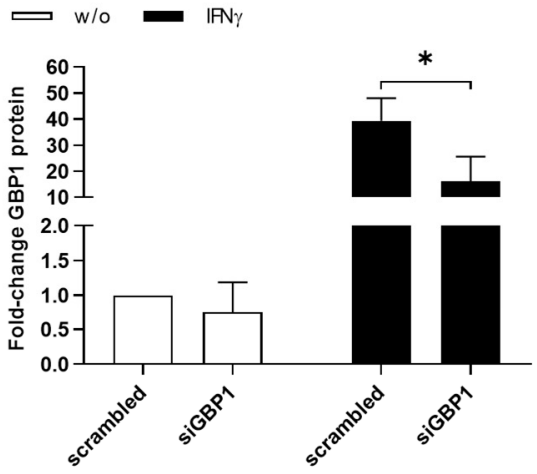

C

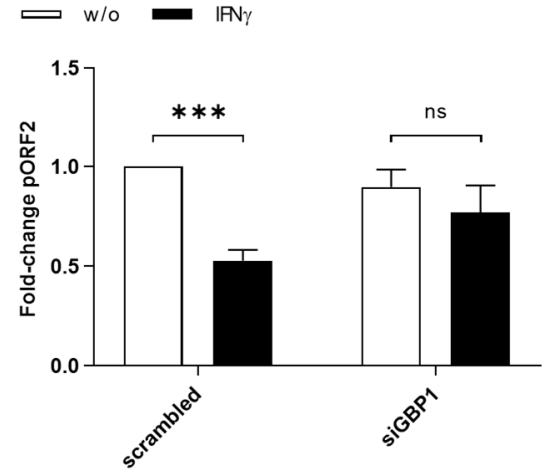

D

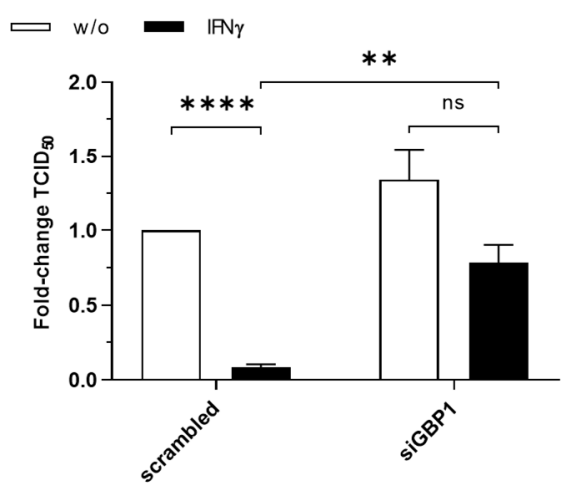

Figure 28. IFN $\gamma$-mediated impairment of HEV life cycle is abrogated upon GBP1 silencing. (A) Representative Western blot of HEV pORF2, GBP1 and GAPDH in total lysates of scrambled siRNA transfected or GBP1 siRNA transfected uninfected or infected cells without and with $100 \mathrm{U} / \mathrm{mL}$ IFN $\gamma$ over $24 \mathrm{~h}$; blue arrow indicates unglycosylated HEV pORF2; grey arrow indicates glycosylated HEV pORF2. (B) Relative change in GBP1 signal intensity of persistently HEV-infected A549 cells in (A); values referred to scrambled siRNA transfected, untreated cells. (C) Relative change in HEV pORF2 signal intensity in (A); values referred to scrambled siRNA transfected, untreated, infected cells. (D) Relative change in number of infectious viral particles being released into cell culture 
supernatant of transfected, $24 \mathrm{~h} 100 \mathrm{U} / \mathrm{mL} \mathrm{IFN} \gamma$ treated and infected cells as assessed in an EPDA; values referred to scrambled siRNA transfected, untreated, infected cells. uninf. = uninfected A549/D3; inf. = persistently HEVinfected A549; w/o = no treatment. ${ }^{*} \mathrm{p}<0.05,{ }^{* *} \mathrm{p}<0.01,{ }^{* * *} \mathrm{p}<0.001,{ }^{* * * *} \mathrm{p}<0.0001$, unpaired t-test with Holm-Sidak correction.

As visible in the above-shown Western blot analysis, silencing efficiency in nonstimulated, HEV-infected cells was relatively low. However, once IFN $\gamma$ was applied, silencing efficiency increased significantly, therefore fulfilling experimental purposes (Figure 28A-B). Upon application if IFN $\gamma$, the intracellular amount of HEV pORF2 diminished. Silencing of GBP1 prompted a loss in IFN $\gamma$-mediated reductions in pORF2 (Figure 28A and C). Similarly, IFN $\gamma$-stimulation caused $\mathrm{HEV}$ virion release to drop heavily, yet again silencing of GBP1 substantially abrogated this process (Figure 28D).

Findings set forth suggest that silencing GBP1 is detrimental to an efficient antiviral effect exerted by IFN $\gamma$. To address this in more detail, single-cell analyses were carried out to rid experimental setups from possible transfection efficiency related issues. Just as before, the nature of the IFN-mediated effect was analysed for lysosomal involvement by evaluating co-localisation between HEV pORF2 and LAMP2 (Figure 29). 


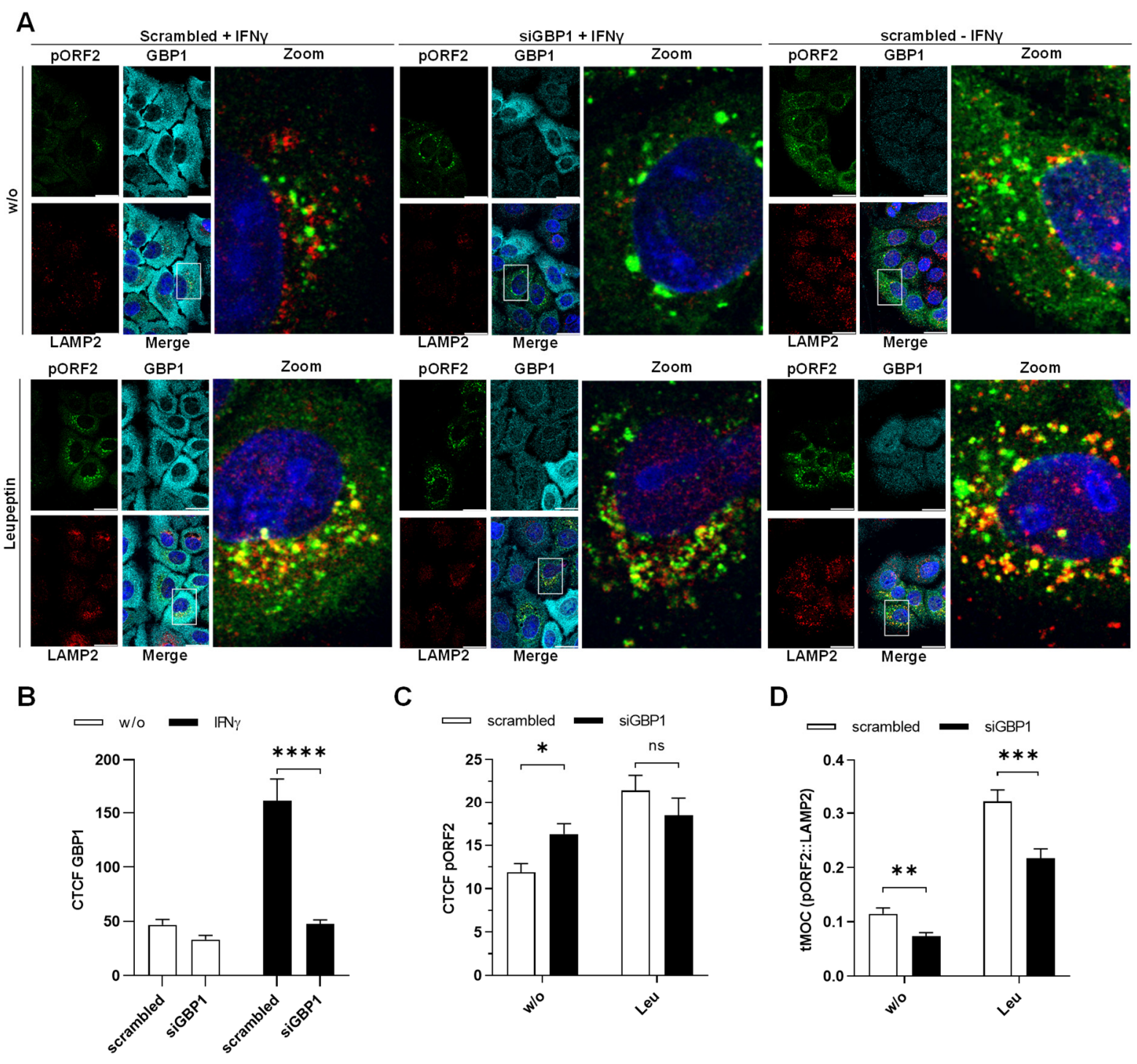

Figure 29. Silencing of GBP1 rescues HEV from IFN $\gamma$-mediated lysosomal localisation and HEV pORF2 reduction. (A) Representative CLSM images of DAPI (blue), HEV pORF2 (green), LAMP2 (red) and GBP1 (cyan) of transfected, persistently HEV-infected A549 cells without or with $200 \mu \mathrm{M}$ Leupeptin over $24 \mathrm{~h}$ and/or $100 \mathrm{U} / \mathrm{mL}$ IFN $\gamma$ treatment over $24 \mathrm{~h}$; scale bar $=20 \mu \mathrm{m}$; zoom represents magnification from area in white rectangle without GBP1 stain. (B) Quantification of GBP1 signal intensity in non-Leupeptin-treated, HEV-infected cells in (A) expressed as CTCF. (C) Quantification of HEV pORF2 signal intensity of IFN $\gamma$-treated, persistently HEV-infected A549 cells in (A) expressed as CTCF. (D) Quantification of pixel co-localisation between HEV pORF2 and LAMP2 in (A) expressed as tMOC. $\mathrm{w} / \mathrm{o}=$ no treatment; Leu $=$ Leupeptin-treatment. $\mathrm{ns}=$ not significant, ${ }^{*} \mathrm{p}<0.05,{ }^{* *} \mathrm{p}<0.01$, ${ }^{* * *} \mathrm{p}<0.001,{ }^{* * * *} \mathrm{p}<0.0001$, unpaired t-test with Holm-Sidak correction.

The majority of cells subjected to GBP1 silencing displayed strongly reduced protein levels even under IFN $\gamma$-stimulation, yet some were not affected (Figure 29A). Notably, transfection efficiency of GBP1 silencing was found to be tremendously higher when compared to overexpression experiments, rendering assumptions based on Western blot analyses (as shown in Figure 28) justified. Based on the above-shown CLSM analyses, siRNA-based silencing led GBP1 levels to drop to non-stimulated levels, even though IFN $\gamma$ was applied (Figure 29B). The latter again caused intracellular HEV pORF2 levels to decline, which was revertible by applying a Leupeptin-treatment. 
Silencing of GBP1 similarly elevated HEV pORF2 levels significantly, rescuing it from degradation (Figure 29A and C). Concordantly, IFN $\gamma$ only was capable to trigger lysosomal localisation of HEV pORF2, if GBP1 was present in sufficient amounts. Once silencing was applied, co-localisation of HEV and lysosomes was significantly reduced (Figure 29D).

In addition to samples being presented as two-dimensional CLSM images, threedimensional reconstructions were generated from the above-shown experimental setting via scanning samples with iterative z-pane shifts. This helps to visualise whether HEV pORF2 simply is in close proximity to LAMP2 or within structures formed by the latter (Figure 30).

A

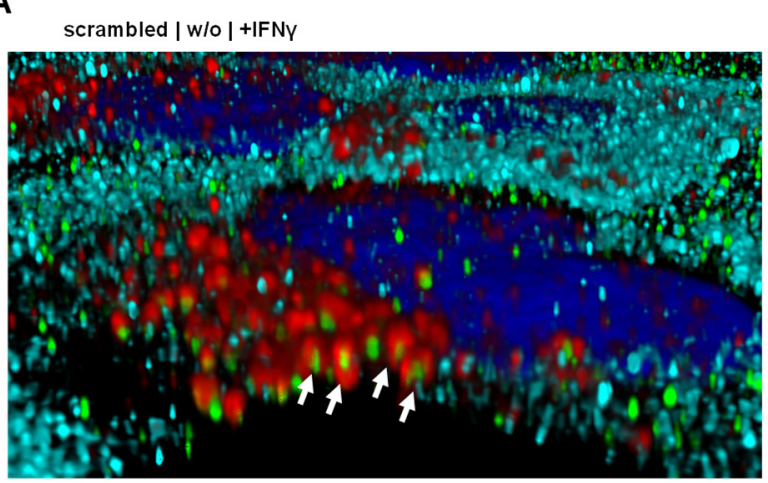

DAPI / PORF2 // LAMP2 // GBP1

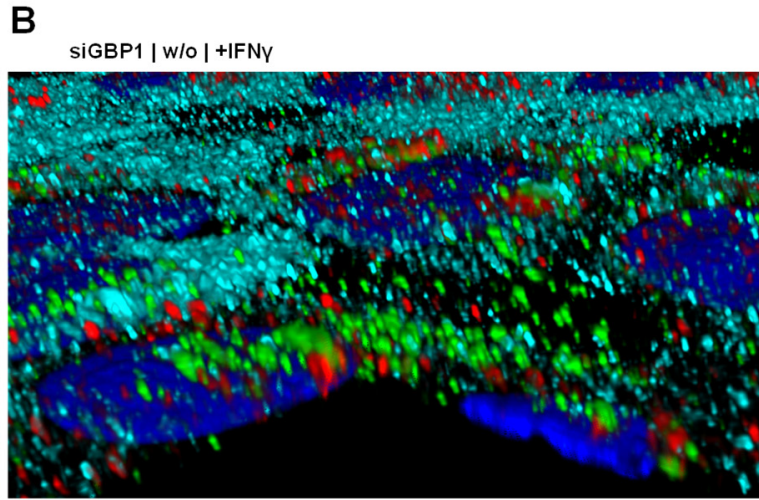

DAPI // pORF2 // LAMP2 // GBP1

Figure 30. IFN $\gamma$-mediated lysosomal HEV encapsulation is abrogated upon GBP1 silencing. (A-B) Representative 3D-reconstruction of z-pane scanning CLSM images of DAPI (blue), HEV pORF2 (green), LAMP2 (red) and GBP1 (cyan) of transfected, persistently HEV-infected A549 cells upon treatment with $100 \mathrm{U} / \mathrm{mL}$ IFN $\gamma$; white arrows indicate pORF2 being present inside LAMP2-harboring structures; 3D volumes were clipped in the $\mathrm{x} / \mathrm{y}$-pane to visualise the interior of lysosomal structures. Both images represent examples from samples presented in Figure 29. w/o = no Leupeptin-treatment.

A stimulation of HEV-infected cells with IFN $\gamma$ resulted in formation of spherical structures carrying LAMP2, indicating that these represent lysosomes. Within these structures, signals of HEV pORF2 could be found, effectively showing the majority of HEV pORF2 residing therein (Figure 30A). Silencing of GBP1 not only reduced the amount of lysosomal clusters, yet it also abrogated the incorporation of HEV pORF2 into these (Figure 30B).

With respect to these data, it presents evident that IFN $\gamma$, similar to a GBP1 overexpression, triggers a GBP1-dependent antiviral effect against HEV that is exerted via lysosomal degradation. 


\subsection{IFN $\gamma$ induces lysosomal cholesterol accumulation}

In the preceding sections, IFNs were proven to impact lysosomal processes with regard to host-defence mechanisms targeting HEV. Such processes are strongly intertwined with lipid homeostasis and -metabolism. Similarly, the entirety of the endosomal system strongly depends on lipid-related processes, especially on cholesterol with regards to their maturation, activity, and functionality. Together they form the endolysosomal system, representing a central point in regulation of cholesterol-homeostasis. On the other hand, HEV represents a virus being entirely dependent on the endosomal system, for its release requires MVBs. Therefore, IFNrelated effects were analysed for their involvement in cholesterol-homeostasis, as they evoke a variety of changes within the cellular continuum. Here, uninfected and HEVinfected cells were treated with IFN $\gamma$ and gene-expression of some central genes in cholesterol-homeostasis were analysed via qPCR (Figure 31).

A

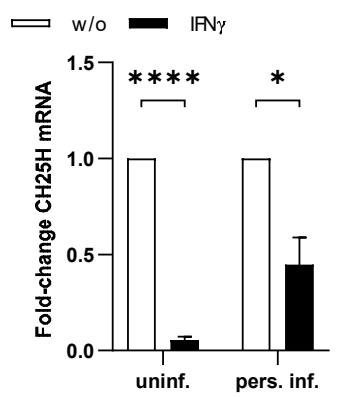

B

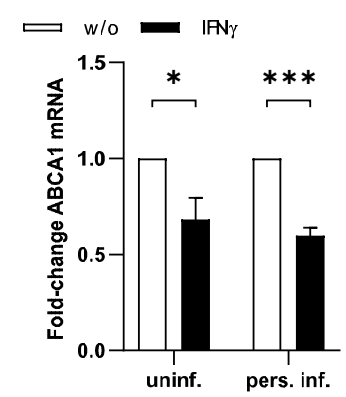

C

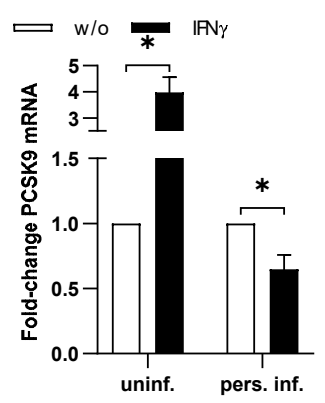

D

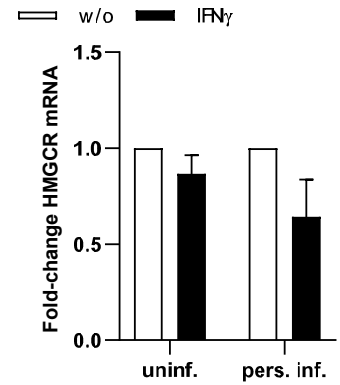

Figure 31. IFN $\gamma$ induces changes in expression of genes encoding proteins involved in maintenance of cholesterol-homeostasis. (A-D) Relative change in intracellular CH25H, ABCA1, PCSK9 or HMGCR mRNA in uninfected or infected cells upon treatment with $100 \mathrm{U} / \mathrm{mL}$ IFN $\gamma$ over $24 \mathrm{~h}$ as assessed via RT-qPCR; values referred to uninfected, untreated cells. uninf. = uninfected A549/D3; inf. = persistently HEV-infected A549; w/o = no treatment. ${ }^{*} \mathrm{p}<0.05,{ }^{* * *} \mathrm{p}<0.001,{ }^{* * * *} \mathrm{p}<0.0001$, unpaired t-test with Holm-Sidak correction.

Within this screening, both cholesterol 25-hydroxylase $(\mathrm{CH} 25 \mathrm{H})$ and ABCA1 mRNA levels were found to be significantly reduced upon IFN $\gamma$-stimulation (Figure 31A-B). Both are involved in cholesterol-detoxification. Further, HMGCR was negatively affected, yet to a lesser extent (Figure 31D). Interestingly, the expression of PCSK9 was found to be significantly elevated in uninfected cells yet reduced in persistently HEVinfected cells (Figure 31C).

As IFN $\gamma$ extorted a strong dysregulation of cholesterol-related genes, a general elevation of intracellular cholesterol could be in place. To assess whether altered geneexpression upon IFN-stimulation really affects intracellular cholesterol concentration and distribution, uninfected and infected A549 cells were subjected to IFN $\alpha$, IFN $\beta$ and IFN $\gamma$ over the course of $24 \mathrm{~h}$. Subsequently, cells were subjected to CLSM analyses 
with cholesterol being visualised by a Filipin III stain. Both the intracellular cholesterol amount and lysosomal localisation of cholesterol was assessed (Figure 32).

A
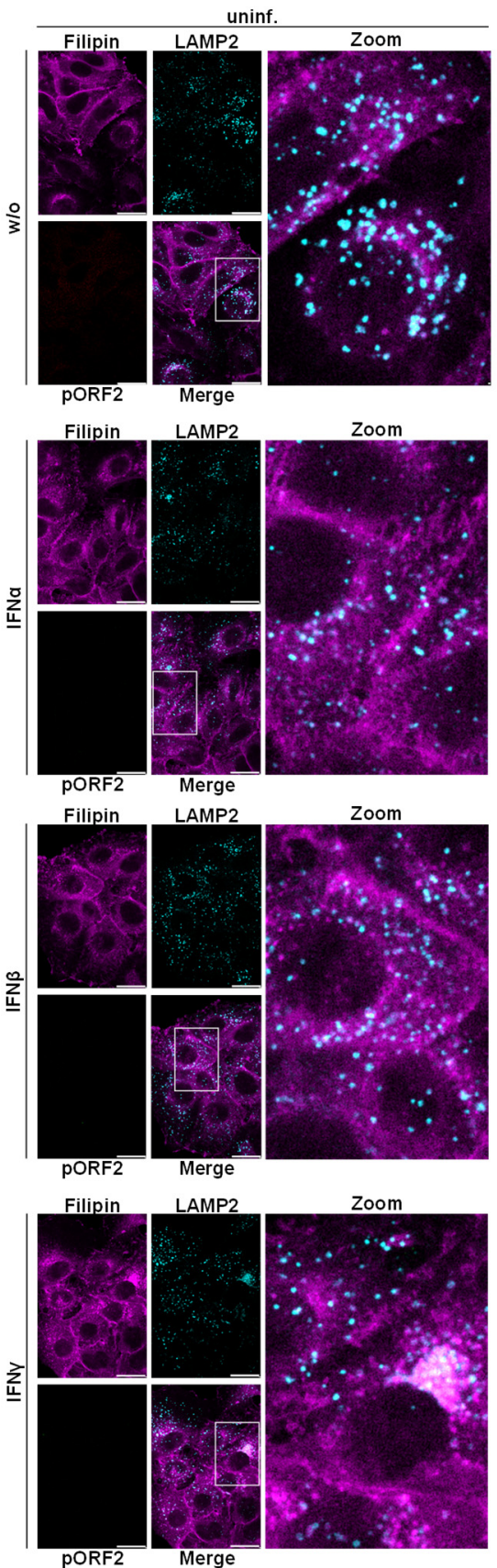

Zoom
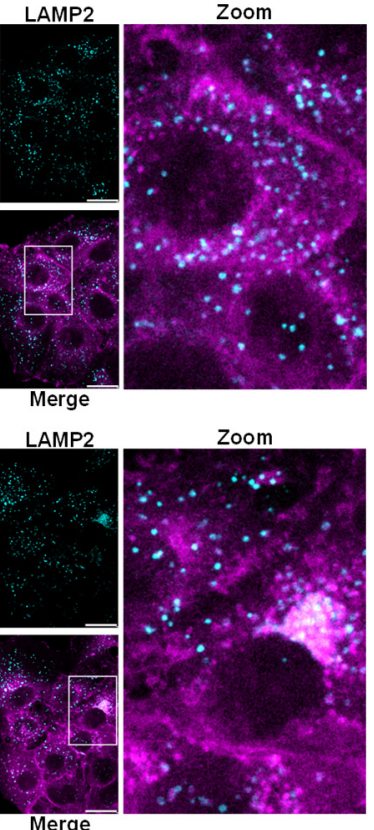

Zoom

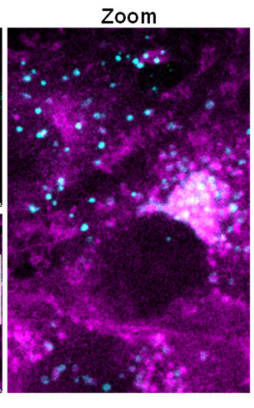

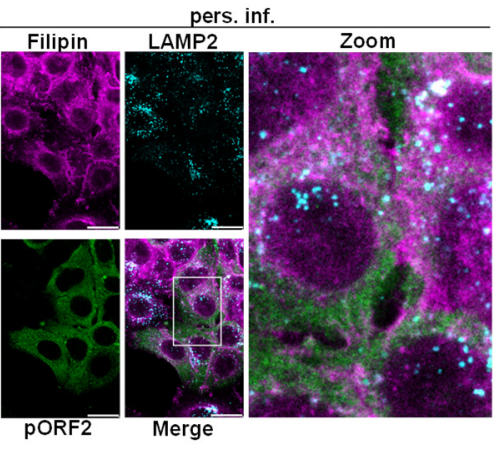
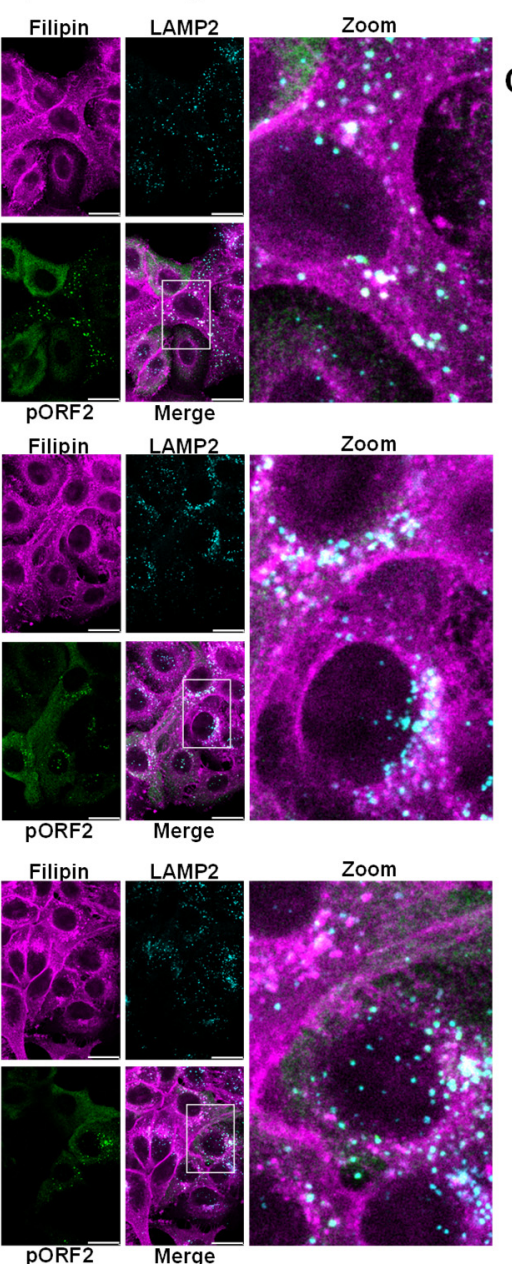

B

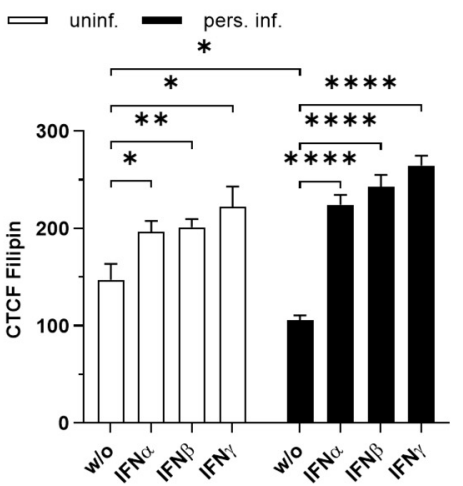

C

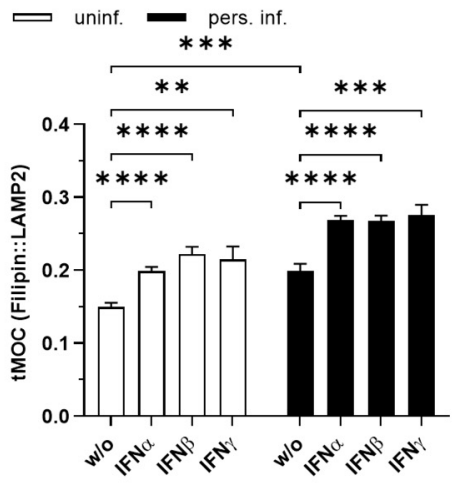

Figure 32. Type I and II Interferons elevate intracellular cholesterol and lead to its lysosomal accumulation. (A) Representative CLSM images of Filipin III (magenta), HEV pORF2 (green) and LAMP2 (cyan) of uninfected or infected cells without or with $100 \mathrm{U} / \mathrm{mL}$ IFN treatment over $24 \mathrm{~h}$; scale bar $=20 \mu \mathrm{m}$; zoom represents magnification from area in white rectangle. (B) Quantification of Filipin III signal intensity in (A) expressed as CTCF. (C) Quantification of pixel co-localisation between Filipin III and LAMP2 in (A) expressed as tMOC. uninf. $=$ uninfected A549/D3; inf. = persistently HEV-infected A549; w/o = no treatment. ${ }^{*} \mathrm{p}<0.05,{ }^{* *} \mathrm{p}<0.01,{ }^{* * *} \mathrm{p}<0.001,{ }^{* * * *} \mathrm{p}<0.0001$, unpaired t-test with Holm-Sidak correction.

All of the tested IFNs markedly elevated intracellular cholesterol levels (Figure 32AB). This effect was predominant in cells subjected to IFN $\gamma$. In general, a big proportion 
of the cellular cholesterol was found to be present within the plasma membrane of untreated cells. However, some intracellular, dot-like accumulations could be observed. These were increased in number upon subjection to IFNs. Interestingly, the globular cholesterol-containing structures were overlapping with globular LAMP2 signals. In fact, once IFN was present, cholesterol increasingly accumulated in LAMP2containing structures, which indicated an increase in lysosomal cholesterol concentration (Figure 32A and C). This effect was more pronounced in HEV-infected cells.

Altogether, these data provide evidence for IFN-related transcriptional changes effectively leading to a lysosomal accumulation of cholesterol. Conspicuously, both the effect on cholesterol and the antiviral effect are most pronounced for IFN $\gamma$. This relationship calls attention to a possible involvement of cholesterol in the antiviral effect against HEV.

5.9. HEV-infection alters expression of cholesterol-regulating genes and reduces intracellular cholesterol

A more detailed assessment of cholesterol-related gene-expression and intracellular cholesterol levels seemed appropriate, based on the before presented data. To do so, a comparative transcriptome profiling was performed analysing differences between uninfected and HEV-infected cells. Results were subsequently validated by reanalysing some of the best hits via qPCR. Additionally, cholesterol levels in uninfected and infected cells were monitored via CLSM analyses (Figure 33). 
A

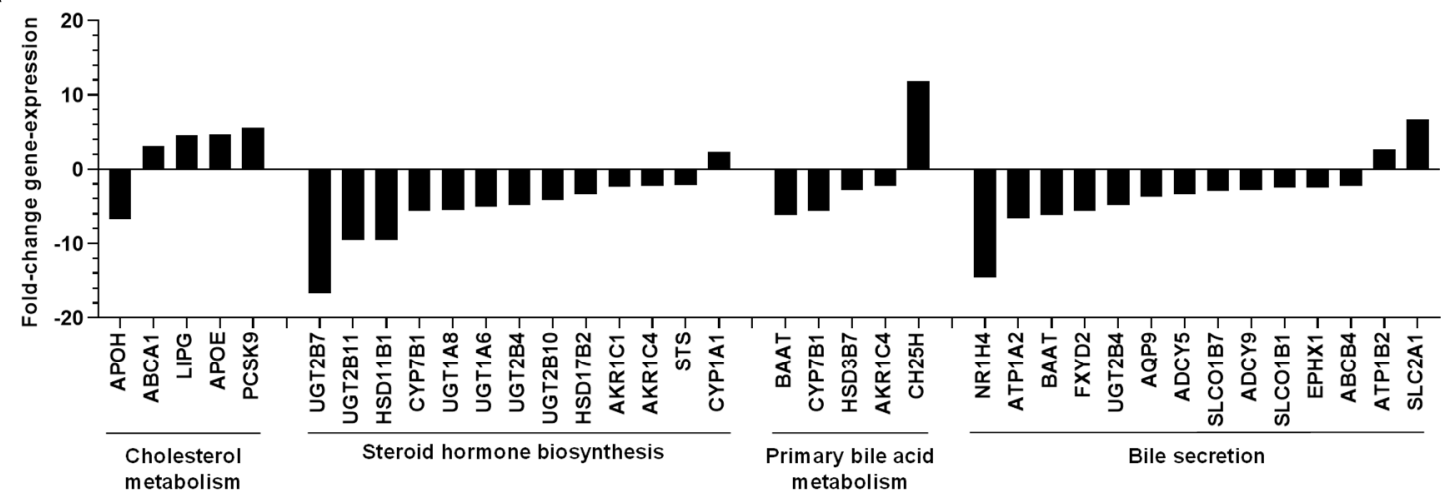

B

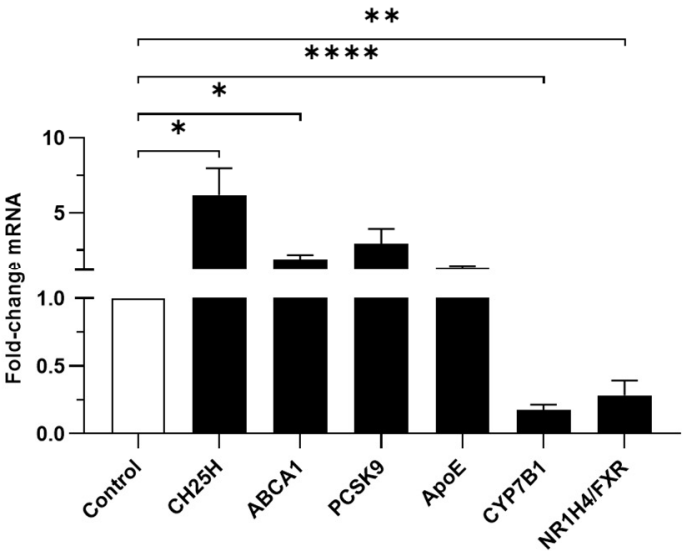

C

uninf.

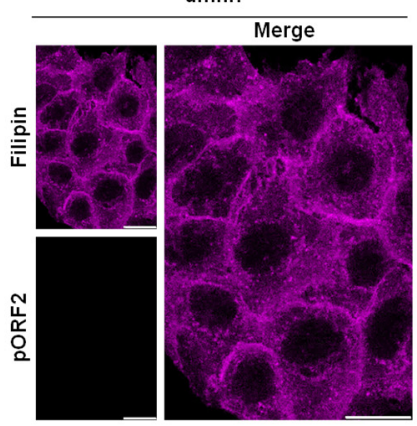

pers. inf.

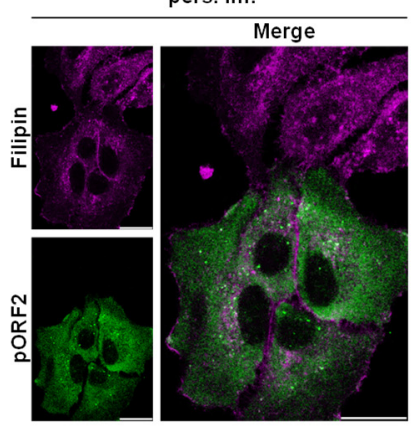

D

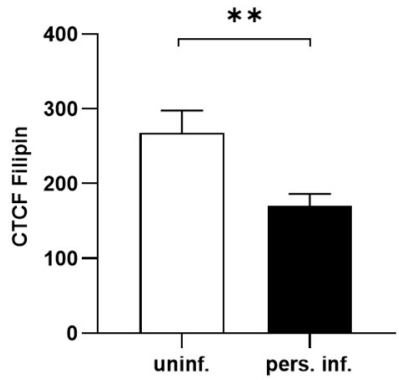

Figure 33. HEV-infection triggers changes in cholesterol-related gene-expression and a reduction in intracellular cholesterol. (A) Representative, relative changes in cholesterol-related transcriptome of uninfected or infected cells, values referred to uninfected cells. (B) Relative change in intracellular CH25H, ABCA1, PCSK9, ApoE, CYP7B1 and NR1H4/FXR mRNA in uninfected or infected cells as assessed via RT-qPCR as validation for (A); values referred to uninfected cells. (C) Representative CLSM images of Filipin III (magenta) and HEV pORF2 (green) of uninfected or infected cells; scale bar $=20 \mu \mathrm{m}$. (D) Quantification of Filipin III signal intensity in (C) expressed as CTCF. uninf. $=$ uninfected A549/D3; inf. = persistently HEV-infected A549. ${ }^{*} \mathrm{p}<0.05,{ }^{* *} \mathrm{p}<0.01,{ }^{* * * *} \mathrm{p}<0.0001$, unpaired t-test with Holm-Sidak correction.

Among other affected pathways and cellular processes, a substantial amount of dysregulated genes was found to be correlated with cholesterol-homeostasis upon HEV-infection (Figure 33A). Some of these genes were picked for qPCR-validation. Herein, the majority of genes encode for proteins being involved in cellular cholesterol-detoxification, namely $\mathrm{CH} 25 \mathrm{H}, \mathrm{ABCA} 1, \mathrm{PCSK} 9$, apolipoprotein E (ApoE) and 25-hydroxycholesterol 7-alpha-hydroxylase (CYP7B1), all of which, except for the 
latter, were found to be enforced in expression in HEV-infected cells (Figure 33B). FXR, being involved in cholesterol-clearance, is the exception to this (Figure 33B). Cohesively, HEV-infected cells were demonstrated to harbour significantly reduced levels of the lipid, when compared to uninfected cells (Figure 33C-D).

In sum, an ongoing HEV-infection results in altered gene-expression of cholesterolregulating genes. This effectively causes intracellular cholesterol levels to decline. At this point, it could be thought of a certain benefit HEV experiences, once there is less cholesterol present in cells, as it displays the opposite situation to an antiviral IFNstimulation.

\subsection{Serum-lipids are reduced in chronically HEV-infected patients}

Persistently HEV-infected A549 cells may represent a sufficient model for most in vitro studies of the virus, yet especially systemically regulated cholesterol levels may differ heavily when compared to an in vivo situation. Thus, serum-lipid concentration of renal transplant patients being diagnosed for an $\mathrm{HEV}$-infection were analysed retrospectively to validate previous experiments. Sera were collected during routine follow-up visits and included in the analysis if infection occurred during this period of time. Selected time points were then grouped as the last follow-up before positive testing ( $\mathrm{t}-1)$, at positive testing $(\mathrm{t} 0)$ and the first follow-up after positive testing $(\mathrm{t}+1)$. Herein, sera were monitored for concentrations of LDL-cholesterol (LDL), HDLcholesterol (HDL), triglycerides (TG) and total cholesterol (TC) to demonstrate effects of an early HEV-infection (Figure 34).

A

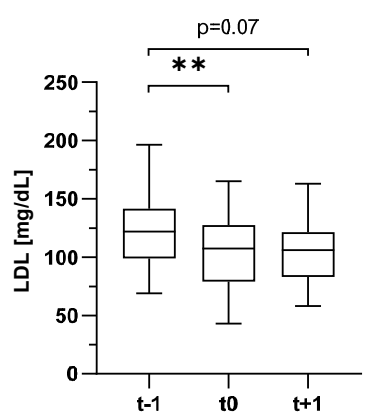

B

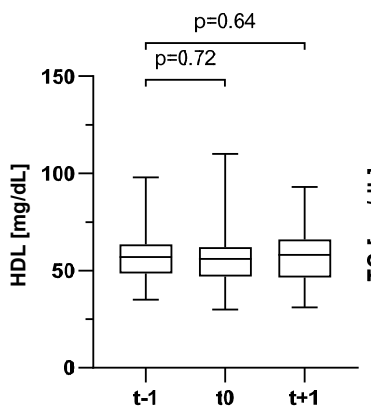

C

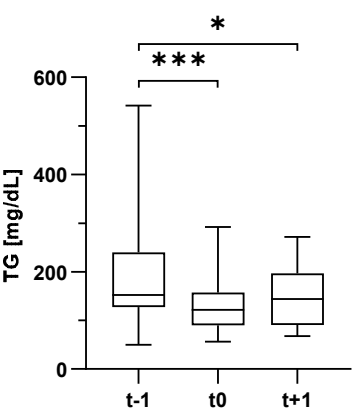

D

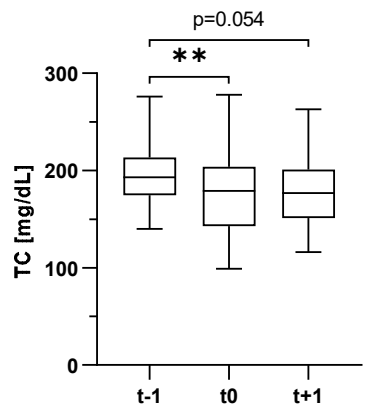

Figure 34. Early HEV-infection triggers reduction of serum-lipids in renal transplant patients. (A-D) Low-density lipoprotein cholesterol (LDL), high-density lipoprotein (HDL), triglyceride (TG) or total cholesterol (TC) concentrations in sera of renal transplant patients before $(t-1)$, at $(t 0)$ or after $(t+1)$ first positive testing for an HEVinfection. $\mathrm{t}-1$ vs. t0: TG $n=29$, TC $n=29$, LDL $n=29$, HDL $n=28$; t0 vs. $\mathrm{t}+1$ : TG $n=20$, TC $n=20$, LDL $n=21$, HDL $n=21$. ${ }^{*} \mathrm{p}<0.05,{ }^{* *} \mathrm{p}<0.01,{ }^{* * *} \mathrm{p}<0.001$, paired, non-parametric t-test.

No HEV-related changes occurred with regards to HDL-concentrations upon infection in patients (Figure 34B). However, LDL, TC and TG concentration significantly 
declined upon the onset of the disease (Figure 34A, C and D). Over a prolonged period of time of up to 6 months between $t 0$ and $t+1$, this reduction is maintained as trend.

These results are in line with findings made in cell culture. Here again, an HEVinfection affects cholesterol concentrations in a negative way, providing a first proof of a more generalised hypothesis.

5.11. A549 cells display a suitable model for studying modulations in cholesterol-

\section{homeostasis}

Following the hypothesis, cholesterol levels are to be modulated in persistently HEVinfected A549 cells. As these represent a pulmonary cell line, the question arose whether they are a suitable model for this experimental setup. Assessment of suitability therefore was performed by subjecting uninfected A549/D3 cells and Huh7 cells, a hepatoma cell line, to 25-hydroxycholesterol (25-HC). Subsequently, changes in expression of cholesterol-related genes were monitored via qPCR (Figure 35).
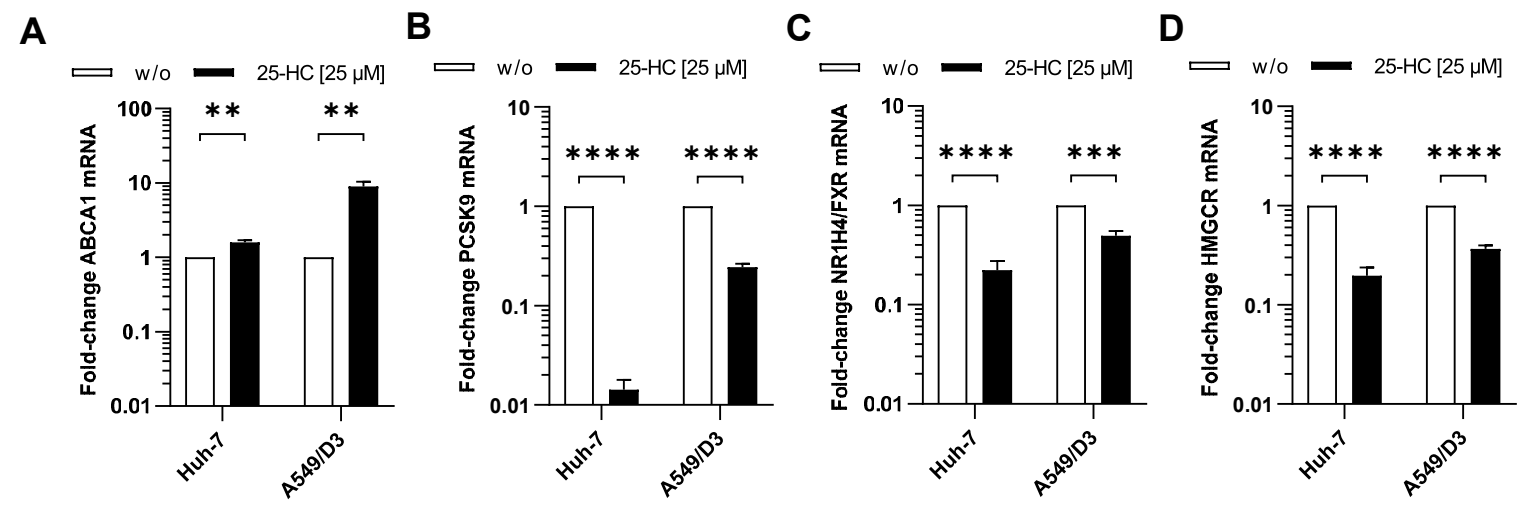

Figure 35. Cholesterol-induced gene-expression in A549 cells behaves similar to Huh-7 cells. (A-D) Relative change in intracellular ABCA1, PCSK9, NR1H4/FXR or HMGCR mRNA in uninfected A549/D3 or Huh-7 cells upon treatment with $25 \mu \mathrm{M} 25-\mathrm{HC}$ over $24 \mathrm{~h}$ as assessed via RT-qPCR; values referred to untreated Huh-7 cells. w/o = no treatment. ${ }^{* *} \mathrm{p}<0.01,{ }^{* * *} \mathrm{p}<0.001,{ }^{* * * *} \mathrm{p}<0.0001$, unpaired t-test with Holm-Sidak correction.

Treatment with 25-HC generally led to heavy changes in gene-expression. Of general interest was the testing for similar behaviour of A549 cells compared to Huh-7 cells. Both cell lines displayed altered gene-expression to a different extent, yet the overall effect, reduction or increase, was similar (Figure 35A-D). This in turn means that A549 cells indeed represent a suitable cell culture system to study cholesterol-homeostasis.

A further step of analysing the system was achieved by screening several compounds to modulate intracellular cholesterol levels. Here, persistently HEV-infected A549 cells were treated with LDL or 25-HC to yield increased cholesterol levels or with Simvastatin to induce the opposite effect via inhibition of cholesterol biosynthesis. Intracellular cholesterol levels as well as cell viability was assessed vie CLSM analyses or viability assay, respectively (Figure 36). 
A

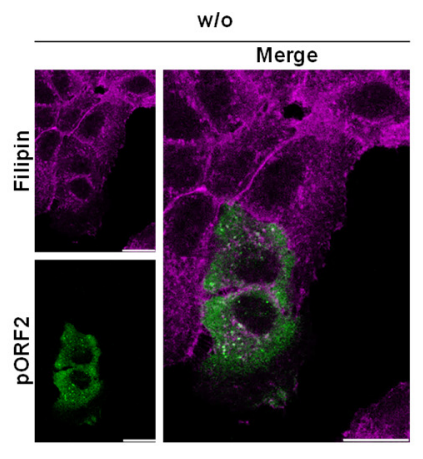

DMSO

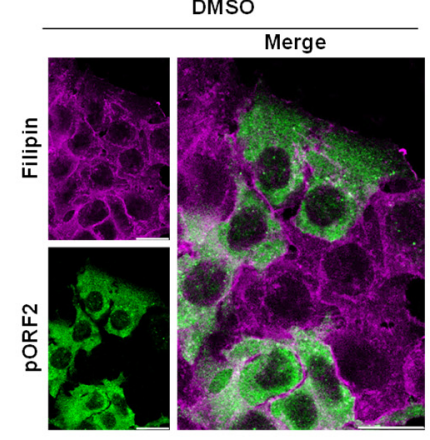

C

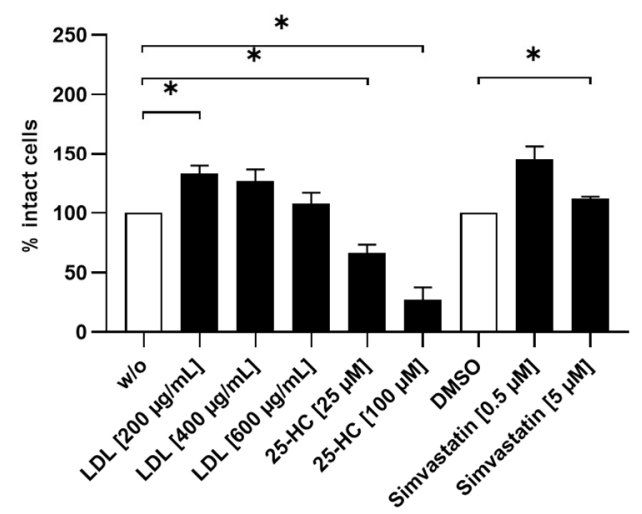

LDL $[600 \mu \mathrm{g} / \mathrm{mL}]$

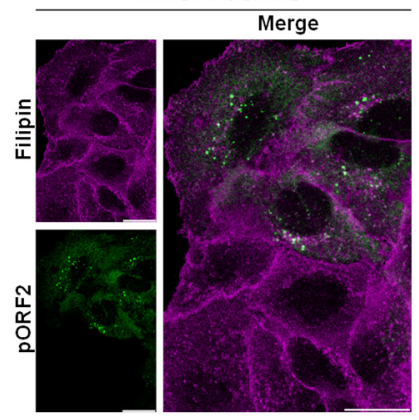

Simvastatin [5 $\mu \mathrm{M}]$

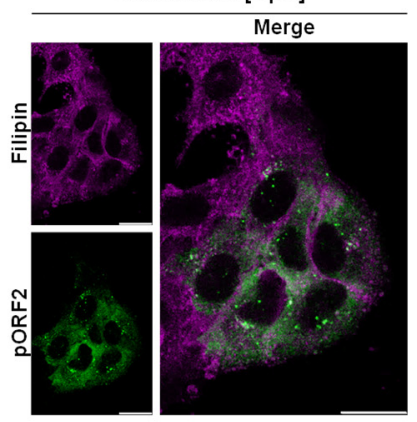

25-HC [25 $\mu \mathrm{M}]$

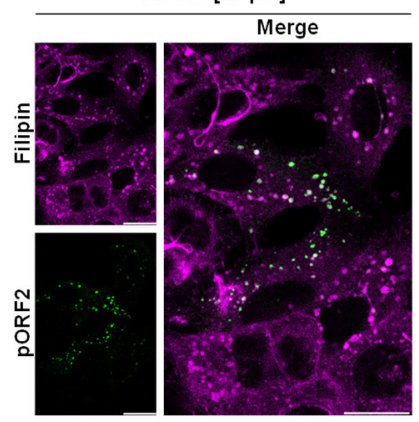

B

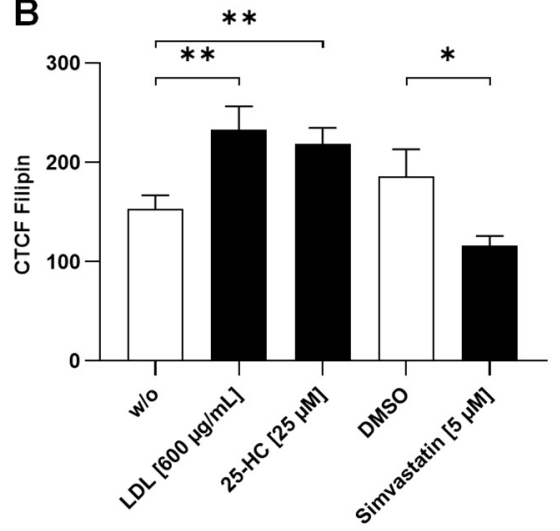

D

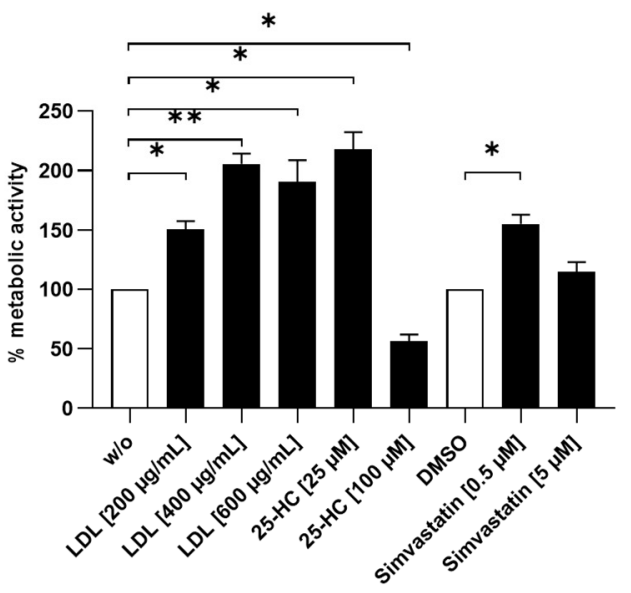

Figure 36. Intracellular cholesterol can be modulated with LDL, 25-HC or Simvastatin in A549 cells. (A) Representative CLSM images of Filipin III (magenta) and HEV pORF2 (green) of $48 \mathrm{~h}$ treated, persistently HEVinfected A549 cells; scale bar $=20 \mu \mathrm{m}$. (B) Quantification of Filipin III signal intensity in (A) expressed as CTCF. (C) Cell viability of persistently HEV-infected A549 cells upon $48 \mathrm{~h}$ treatment with LDL, 25-HC or Simvastatin as assessed via an LDH assay; values expressed as \% intact cells referred to the experimental control. (D) Cell viability of persistently HEV-infected A549 cells upon $48 \mathrm{~h}$ treatment with LDL, 25-HC or Simvastatin as assessed via a PrestoBlue assay; values expressed as \% metabolic activity referred to the experimental control. $\mathrm{w} / \mathrm{o}=$ no treatment. ${ }^{*} \mathrm{p}<0.05,{ }^{* *} \mathrm{p}<0.01$, unpaired t-test with Holm-Sidak correction.

Both a 25-HC- and LDL-treatment, augmented cellular cholesterol levels. Concordantly, treatment with Simvastatin yielded a reduction in the latter (Figure 36A-B). Especially in 25-HC treated cells, cholesterol appeared to accumulate in dotlike structures (Figure 36A). As per the viability assays, none of the applied treatments induced major cell death, with the exception of $100 \mu$ M 25-HC (Figure 36C). Similarly, 
this treatment was the sole one to display a heavily reduced redox metabolism (Figure 36D) and was excluded from major functional experiments.

Presented data in this section demonstrates that A549 cells are a suitable system for studying cholesterol-homeostasis in cell culture. Additionally, the results provide evidence that elevation and reduction of intracellular cholesterol is efficient with the applied treatments.

\subsection{Elevation of intracellular cholesterol restricts HEV}

Before-shown data indicate that intracellular cholesterol levels may inhibit the life cycle of HEV. As modulation of cellular cholesterol was efficient in A549 cells, consequences for HEV were analysed within these. Therefore, persistently HEVinfected A549 cells were treated with LDL and 25-HC or Simvastatin over $48 \mathrm{~h}$ to elevate or decrease cellular cholesterol, respectively. The amount of HEV pORF2 in treated cells was assessed via Western blot analyses (Figure 37).

A

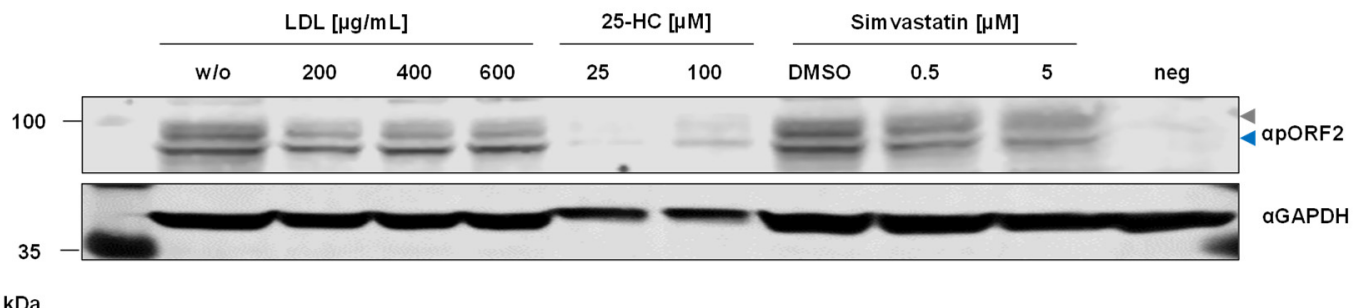

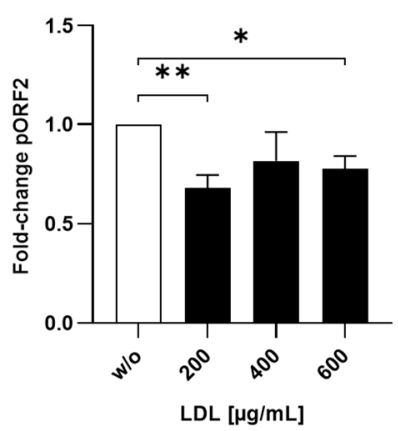

C

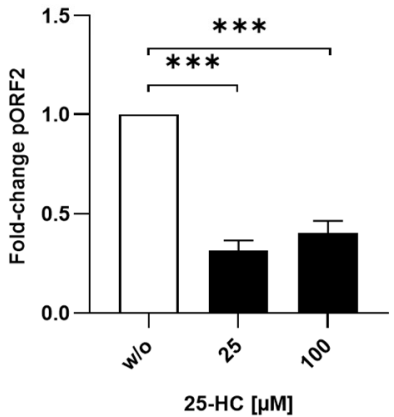

D

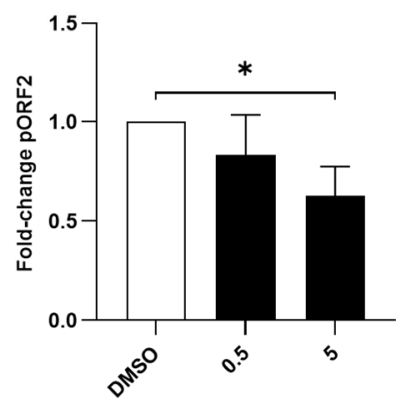

Simvastatin $[\mu \mathrm{M}]$

Figure 37. Increased intracellular cholesterol concentrations lead to reduction in intracellular HEV pORF2. (A) Representative Western blot of HEV pORF2 and GAPDH in total lysates of persistently HEV-infected A549 cells subjected to a $48 \mathrm{~h}$ treatment with LDL, 25-HC or Simvastatin; blue arrow indicates unglycosylated HEV pORF2; grey arrow indicates glycosylated HEV pORF2. (B-D) Relative change in HEV pORF2 signal intensity in (A); values referred to experimental control. w/o $=$ no treatment. ${ }^{*} \mathrm{p}<0.05,{ }^{* *} \mathrm{p}<0.01,{ }^{* * *} \mathrm{p}<0.001,{ }^{* * * *} \mathrm{p}<0.0001$, unpaired t-test with Holm-Sidak correction.

Elevation of cellular cholesterol led to a significant decrease in HEV pORF2 for all tested concentrations of LDL and 25-HC (Figure 37A-C). The same holds true for a reduction in cellular cholesterol via treatment of cells with Simvastatin (Figure 37A and D). Although the slight cytotoxic effect exerted by $25-\mathrm{HC}$ became apparent, 
treatment with the cholesterol-derivative yielded the most prominent reduction in HEV pORF2.

An overall reduction of intracellular HEV pORF2 upon modulation of cellular cholesterol indeed is somewhat ambiguous. Notably, a reduction in viral capsid protein inside infected cells may have several causes, two of which are either enhanced viral release or protein degradation. Thus, viral release was monitored in cells subjected to cholesterol modulation by assessing levels of intra- and extracellular HEV transcripts as well as the amount of released virions via qPCR and EPDA, respectively. To correlate in vitro findings with an in vivo situation, patient data of chronically HEVinfected renal transplant patients receiving statin-treatment were compared to nontreated patients with regards to viral titres in sera. Further, virion density was assessed via isopycnic density-gradient centrifugation to determine whether eHEV or nHEV is being released from cells (Figure 38).

A

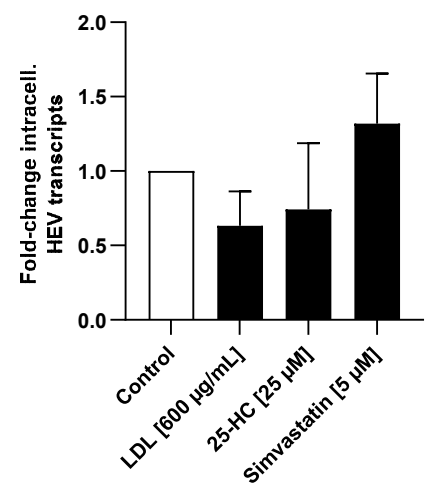

B

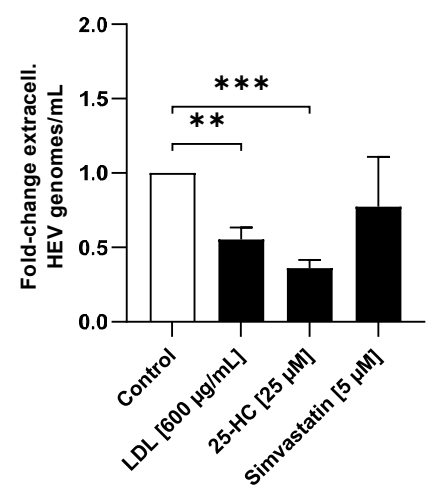

C

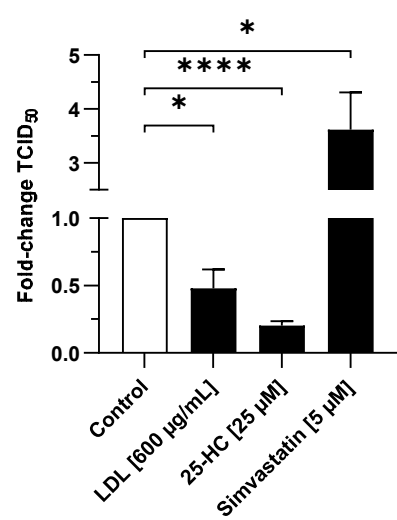

D

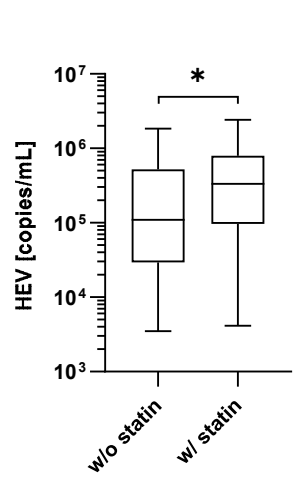

E
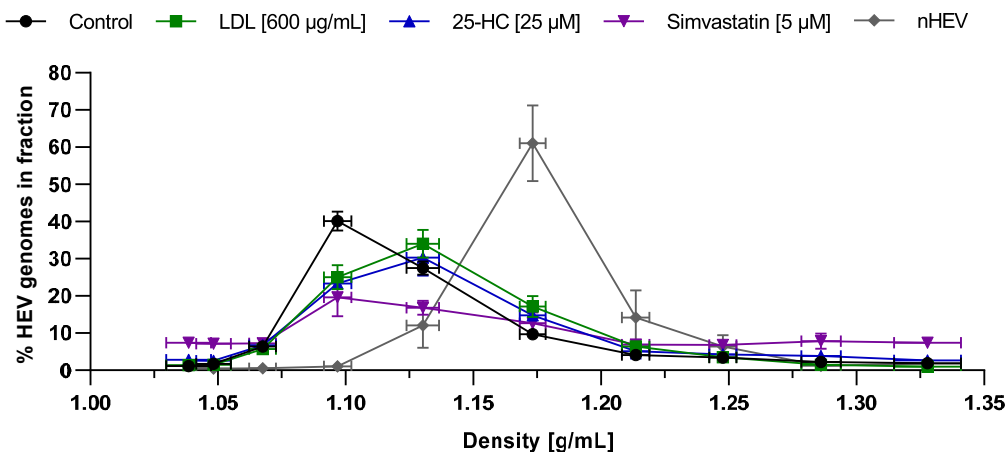

Figure 38. High intracellular cholesterol reduces viral release, whereas low intracellular cholesterol is in favour of it. (A) Relative change in intracellular HEV transcripts in persistently HEV-infected A549 cells upon $48 \mathrm{~h}$ treatment with LDL, 25-HC or Simvastatin as assessed via RT-qPCR; values referred to experimental control. (B) Relative change in extracellular HEV genomes released from persistently HEV-infected A549 cells upon $48 \mathrm{~h}$ treatment with LDL, 25-HC or Simvastatin as assessed via RT-qPCR; values referred to experimental control. (C) Relative change in number of infectious viral particles being released into cell culture supernatant of persistently HEV-infected A549 cells as assessed in an EPDA; values referred experimental control. (D) Concentration of HEV genomes in sera of chronically HEV-infected renal transplant patients without or with statin-treatment; 42 patients with a total of 129 measured values (w/o statin $n=74 ; w /$ statin $n=55)$. (E) Relative amount of HEV genomes found 
in density gradient fractions of HEV virions released by persistently HEV-infected A549 cells upon 48 h LDL, 25HC or Simvastatin treatment, values expressed as \% of genomes in the whole gradient being present in respective fraction; $\mathrm{nHEV}=$ taurocholate-treated virus stock. $\mathrm{w} / \mathrm{o}=$ no treatment; control $=\mathrm{w} / \mathrm{o}$ for LDL and 25-HC or DMSO for Simvastatin. ${ }^{*} \mathrm{p}<0.05,{ }^{* *} \mathrm{p}<0.01,{ }^{* *} \mathrm{p}<0.001,{ }^{* * *} \mathrm{p}<0.0001$, unpaired t-test with Holm-Sidak correction for panel A-D; Mann-Whitney test for panel D.

Analysing intracellular HEV transcripts indicated that there were no major changes upon treatment with either of the substances, yet a reductive trend is visible in LDLtreated cells (Figure 38A). This also proved true for extracellular HEV RNA released from Simvastatin-treated cells. However, in cells with increased cellular cholesterol, viral RNA release was significantly reduced (Figure 38B). This effect was even more pronounced for virion release, where both LDL and 25-HC reduced the number of released infectious viral particles markedly. Interestingly, the opposite was observed for cells with reduced cellular cholesterol upon Simvastatin-treatment, where an enforcement of virion release became apparent (Figure 38C). This, in turn, went in line with the statin-induced effect detected in chronically HEV-infected patients. Here, an application of the drugs significantly increased the viral load found in sera (Figure 38D). A further interesting aspect was found when analysing the density of virions released from treated cells. Both untreated and Simvastatin-treated cells released virions matching exosomal buoyant-density $(\sim 1.08-1.12 \mathrm{~g} / \mathrm{mL})$. Cells with increased cellular cholesterol however, released virions with a relatively higher buoyant-density ( 1.12 - $1.15 \mathrm{~g} / \mathrm{mL}$ ), yet not representing nHEV (Figure 38E).

In summary, reduced cellular cholesterol reduces intracellular HEV pORF2 levels. This is found to be due to an enhanced virion release, effectively causing more pORF2 to be released as part of eHEV. A similar behaviour is found in vivo, suggesting that statintreatment may be counter indicated. Opposing to this, an increase in cellular cholesterol causes both pORF2 in cells and virion release to decline. This indicates that cholesterol-dependent degradative processes could be in place.

\subsection{Elevated intracellular cholesterol induces lysosomal degradation of HEV}

Previously presented data indicated lysosomal degradation to be an important gatekeeper in viral inactivation. Concordantly, cholesterol may herein be a similarly important factor. Hence, the antiviral effect being exerted by increased cellular cholesterol was analysed with respect to lysosomal incorporation of HEV. Again, CLSM analyses were of use to validate above shown Western blot analyses and to assess lysosomal localisation of HEV pORF2 (Figure 39). 
A

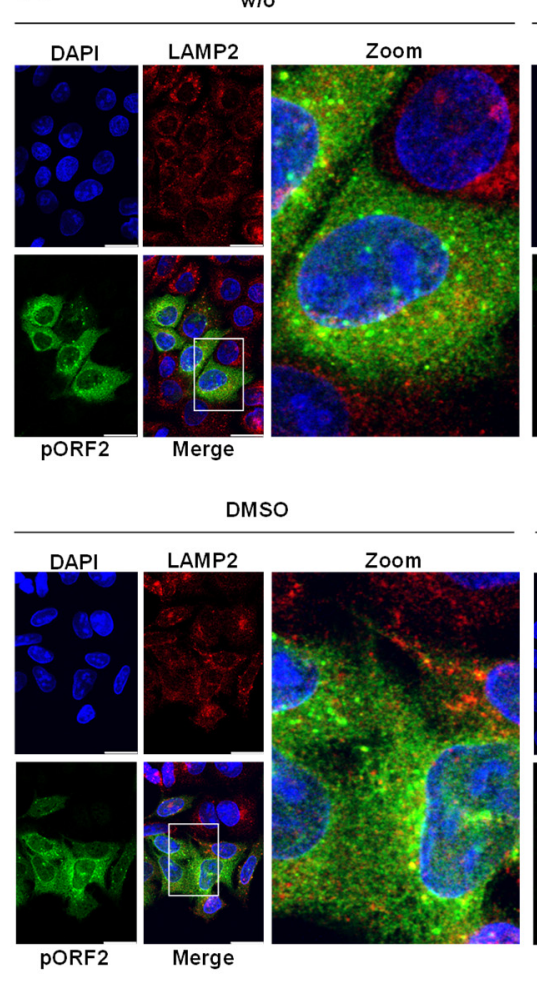

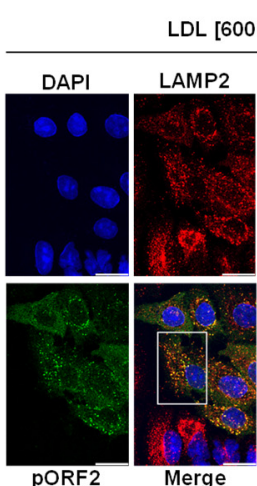

pORF2
$[600 \mu \mathrm{g} / \mathrm{mL}]$

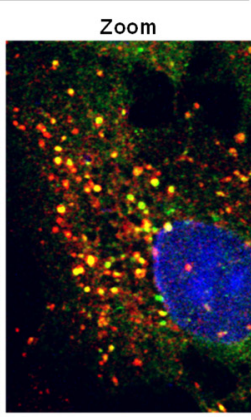

Simvastatin $[5 \mu \mathrm{M}]$

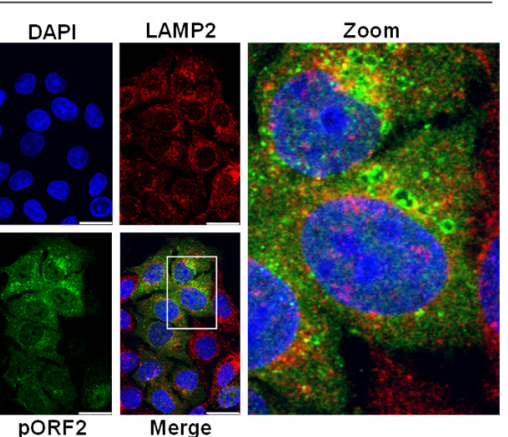

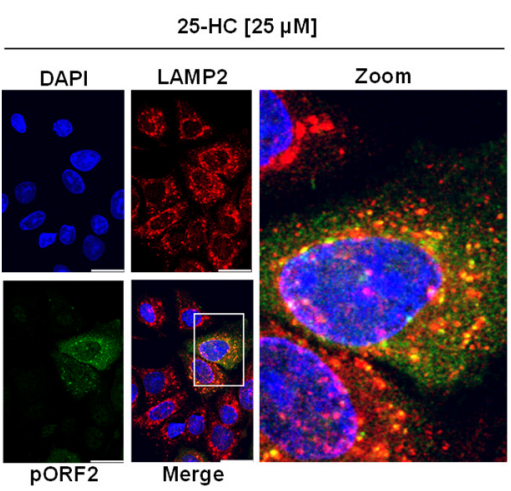

B

C

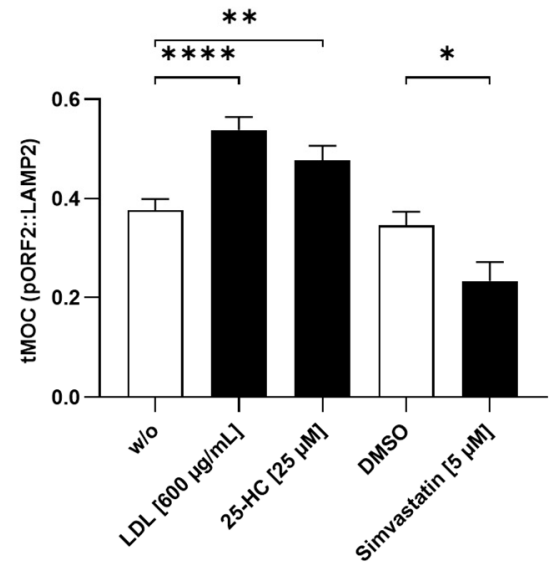

Figure 39. Modulation of intracellular cholesterol reduces intracellular HEV pORF2 and affects its lysosomal localisation. (A) Representative CLSM images of DAPI (blue), HEV pORF2 (green) and LAMP2 (red) of persistently HEV-infected A549 cells under 48 h LDL, 25-HC or Simvastatin treatment; scale bar $=20 \mu \mathrm{m}$; zoom represents magnification from area in white rectangle. (B) Quantification of HEV pORF2 signal intensity in (A) expressed as CTCF. (C) Quantification of pixel co-localisation between HEV pORF2 and LAMP2 in (A) expressed as tMOC. $\mathrm{w} / \mathrm{o}=$ no treatment. ${ }^{*} \mathrm{p}<0.05,{ }^{* *} \mathrm{p}<0.01,{ }^{* * *} \mathrm{p}<0.001,{ }^{* * * *} \mathrm{p}<0.0001$, unpaired t-test with Holm-Sidak correction.

Modulation of intracellular cholesterol led to a significant reduction in HEV pORF2 in single-cell analyses. This effect again was most prominent upon treatment with $25-\mathrm{HC}$ (Figure 39A-B). In both LDL- and 25-HC-treated cells, intracellular pORF2 accumulated in dot-like structures perinuclearly as well as in the cell periphery. The same was observed for the subcellular distribution of LAMP2. These accumulations of both pORF2 and LAMP2 were found to co-localise with signals overlapping 
significantly more as compared to the experimental control. In contrast, a reduction in this co-localisation could be detected in Simvastatin-treated cells (Figure 39A and C).

With high intracellular cholesterol leading to an increase in lysosomal localisation of $\mathrm{HEV}$, lysosomal degradation taking place is evident. A co-treatment with Leupeptin therefore was applied to inhibit this process and CLSM analyses were of use to monitor whether pORF2-degradation could still occur (Figure 40).

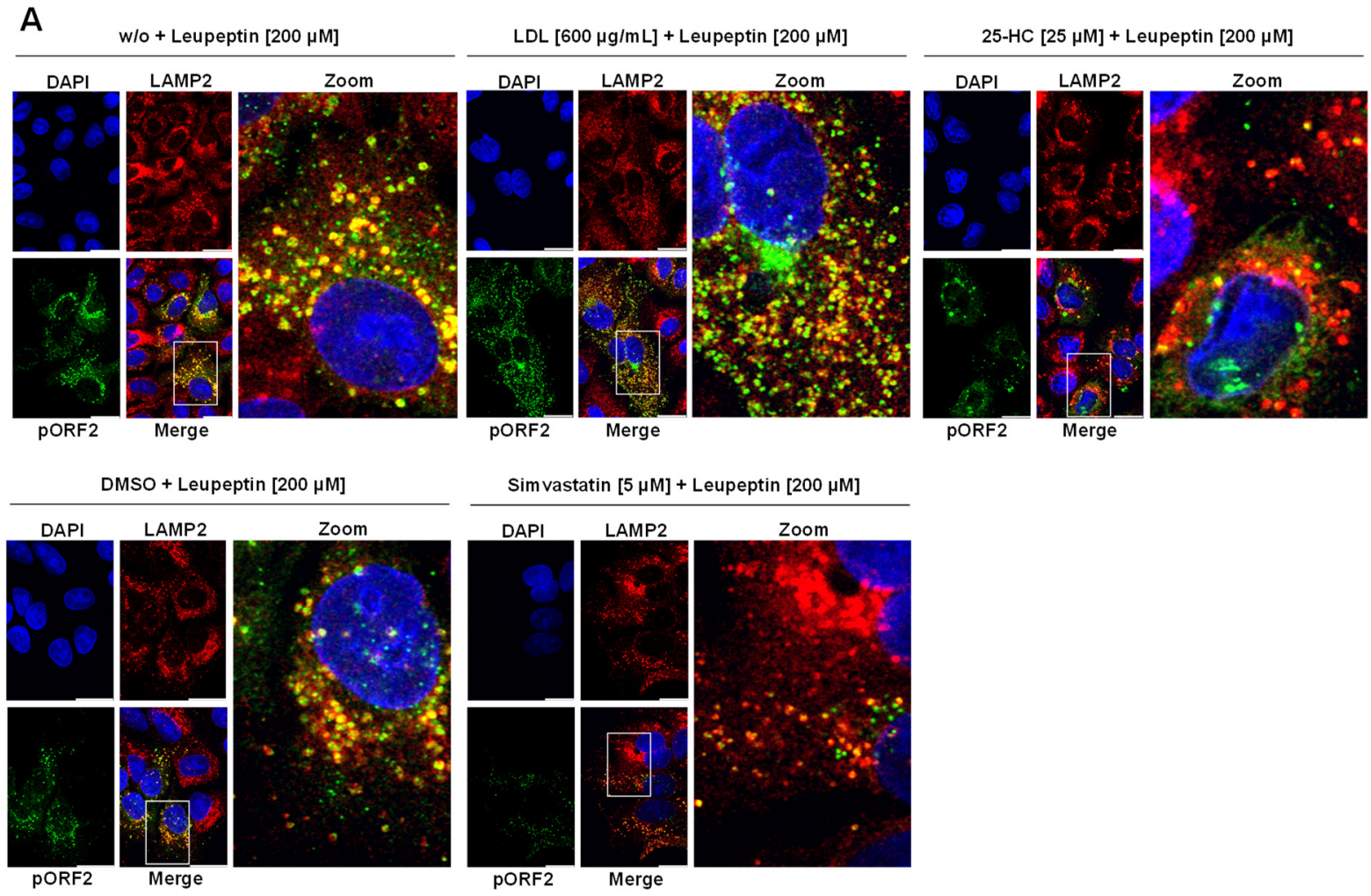

B

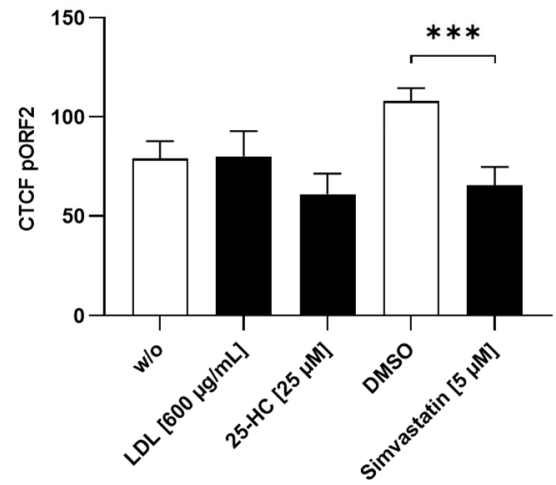

C

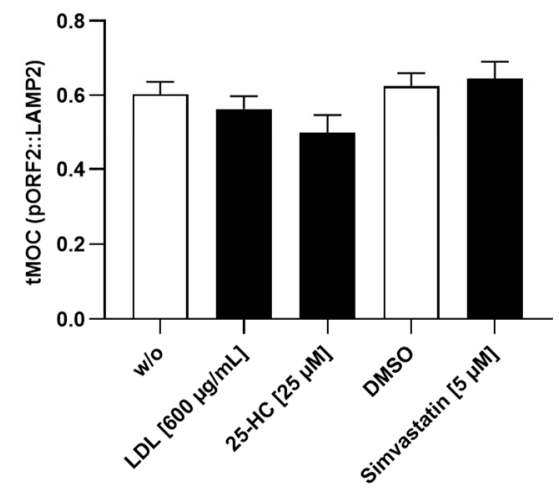

Figure 40. Blocking of lysosomal degradation rescues intracellular HEV pORF2 from cholesterol-induced reduction. (A) Representative CLSM images of DAPI (blue), HEV pORF2 (green) and LAMP2 (red) of persistently HEV-infected A549 cells under 48 h LDL, 25-HC or Simvastatin treatment with $200 \mu \mathrm{M}$ Leupeptin treatment over $24 \mathrm{~h}$; scale bar $=20 \mu \mathrm{m}$; zoom represents magnification from area in white rectangle. (B) Quantification of HEV pORF2 signal intensity in (A) expressed as CTCF. (C) Quantification of pixel co-localisation between HEV pORF2 
and LAMP2 in (A) expressed as tMOC. w/o $=$ no treatment. ${ }^{*} \mathrm{p}<0.05,{ }^{* *} \mathrm{p}<0.01,{ }^{* * *} \mathrm{p}<0.001,{ }^{* * * *} \mathrm{p}<0.0001$, unpaired ttest with Holm-Sidak correction.

Application of Leupeptin on HEV-infected cells led to a pronounced accumulation of pORF2. This clustering was equally pronounced in the different co-treatments and differences in lysosomal localisation of pORF2 was abolished (Figure 40A and C). As per the amount of intracellular pORF2, Simvastatin retained its reductive effect even under inhibition of lysosomal degradation. In contrast, applying Leupeptin on cells with increased cellular cholesterol was detrimental for the previously detected reductions in HEV pORF2. Herein, the co-treatment rescued the pORF2 amount from reduction yielding signal intensities being in a range of the experimental control (Figure 40A-B).

On the whole, increasing cellular cholesterol leads to lysosomal localisation of HEV. This is accompanied by subsequent degradation. Hence, a cholesterol-induced lysosomal degradation of HEV is evident. 


\subsection{Cholesterol-modulating drugs act efficiently in A549 cells}

Several drugs influencing systemic or intracellular cholesterol are available on the market or subject to clinical trials. A selection of these were therefore set to be screened for antiviral activity against HEV. First and foremost, drug-candidates were analysed for potential cytotoxic effects exerted on persistently HEV-infected A549 cells (Figure 41).

A

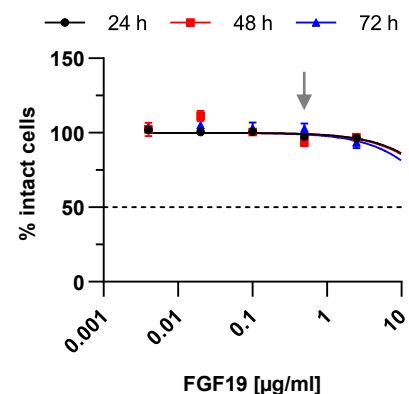

D

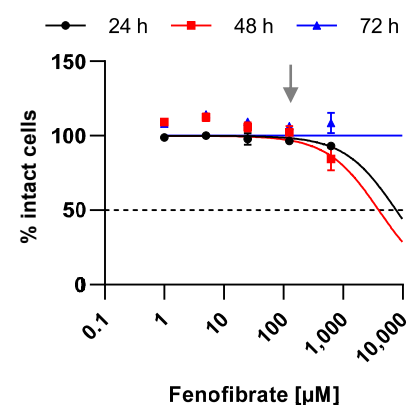

B

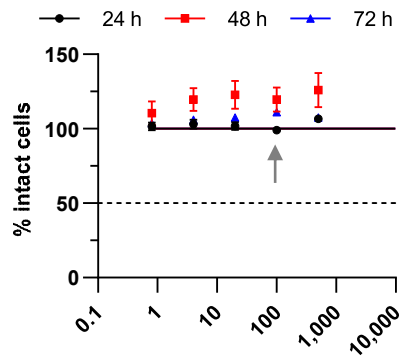

Alirocumab $[\mathrm{ng} / \mathrm{ml}]$

$\mathbf{E}$

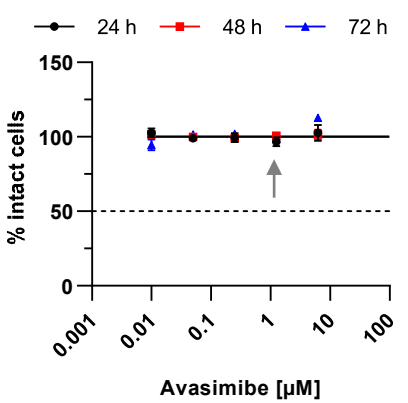

C

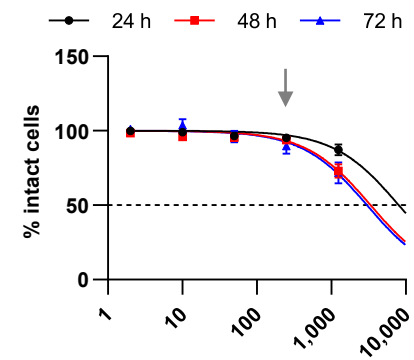

Gemfibrozil $[\mu \mathrm{M}]$

$\mathbf{F}$

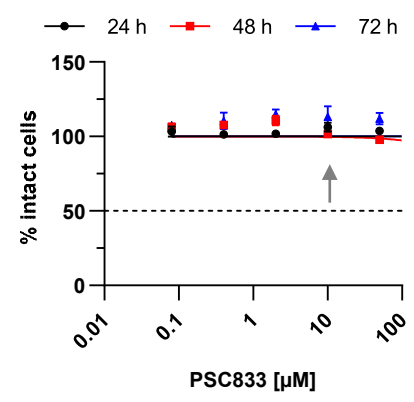

Figure 41. Cholesterol modulating drugs are non-toxic to A549 cells. (A-F) Cell viability of persistently HEVinfected A549 cells upon 24-72 h treatment with FGF19, Alirocumab, Gemfibrozil, Fenofibrate, Avasimibe or PSC833 as assessed via an LDH assay; values expressed as \% intact cells referred to the experimental control; grey arrows indicate highest concentration used for subsequent functional analyses; curves fitted with an inhibitory dose-response model.

None of the tested substances induced major cytotoxicity in a wide spectrum of concentrations between $24-72 \mathrm{~h}$ (Figure 41A-F). Solely exceedingly high concentrations of Gemfibrozil or Fenofibrate led to a slight drop in cellular integrity (Figure 41C-D). Tolerated concentrations of the compounds were therefore considered for further experiments.

Similar to cytotoxicity, cytostaticity of the compounds was to be assessed on persistently HEV-infected A549 cells to rule out detrimental effects on cellular redoxmetabolism (Figure 42). 
A

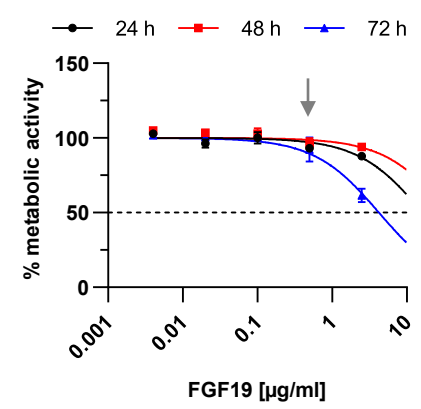

D

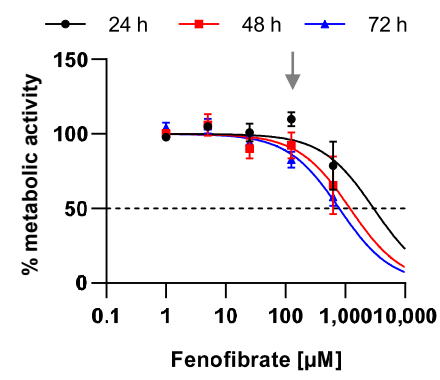

B

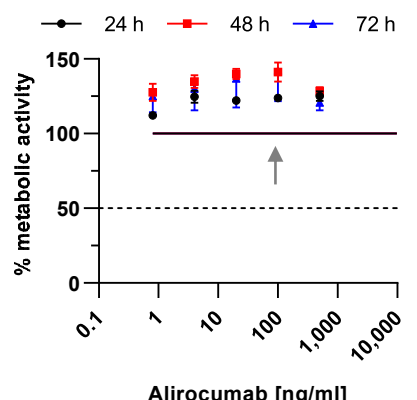

$\mathbf{E}$

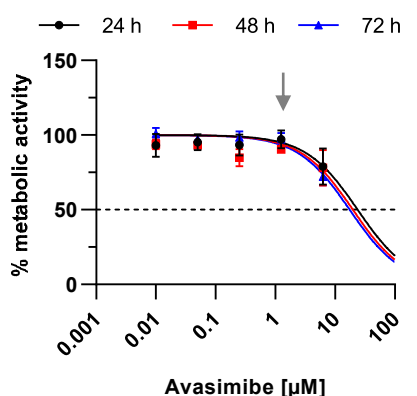

C

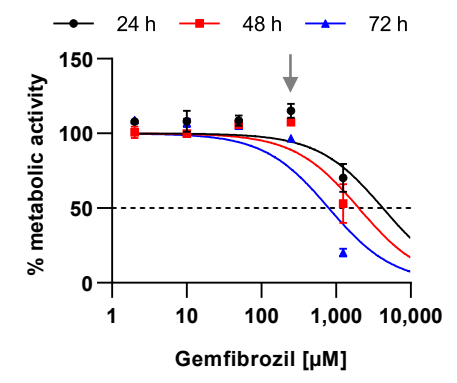

$\mathbf{F}$

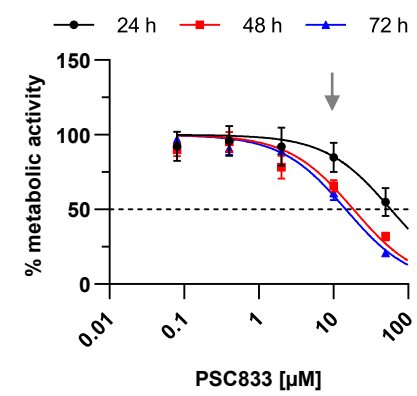

Figure 42. Cholesterol modulating drugs display slight cytostatic effects in A549 cells. (A-F) Cell viability of persistently HEV-infected A549 cells upon 24-72 h treatment with FGF19, Alirocumab, Gemfibrozil, Fenofibrate, Avasimibe or PSC833 as assessed via a PrestoBlue assay; values expressed as \% metabolic activity referred to the experimental control; grey arrows indicate highest concentration used for subsequent functional analyses; curves fitted with an inhibitory dose-response model.

Just like in cytotoxicity assays, only extremely high concentrations of the compounds yielded cytostatic effects (Figure 42A-F). Therefore, only the second highest concentrations were used for subsequent analyses.

The overall aim of using cholesterol-modulating drugs was to elevate intracellular cholesterol levels. Within the hypothesis, this should trigger lysosomal degradation of HEV. Therefore, drug-candidates were tested for their capacity to lead to an accumulation of intracellular cholesterol after $48 \mathrm{~h}$ in persistently HEV-infected A549 cells via CLSM analyses (Figure 43). 
A

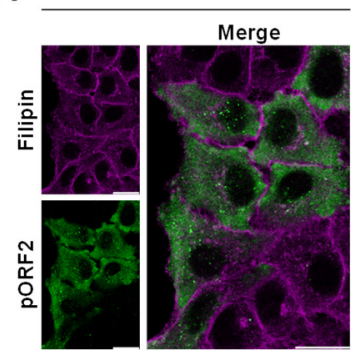

Avasimibe $[1.25 \mu \mathrm{M}]$

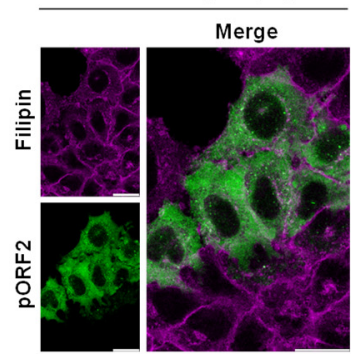

B

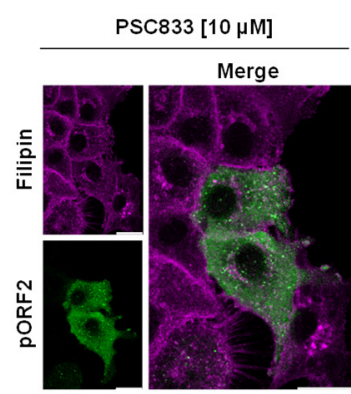

w/o

Merge

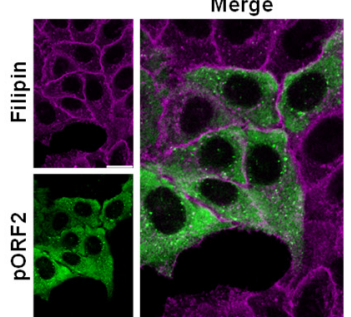

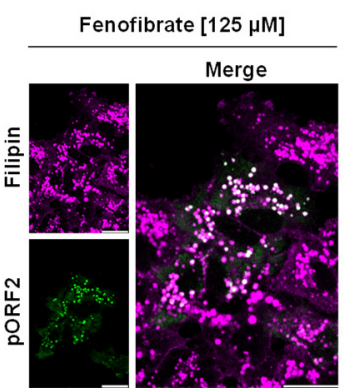

FGF19 $[0.5 \mu \mathrm{g} / \mathrm{mL}]$

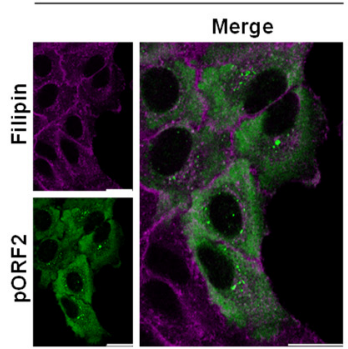

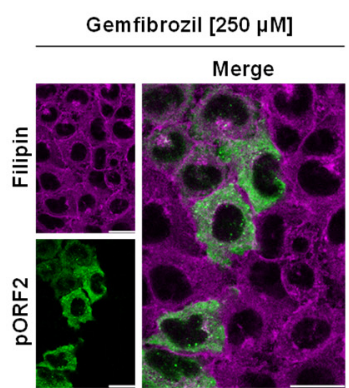

Alirocumab $[0.5 \mu \mathrm{g} / \mathrm{mL}$ ]

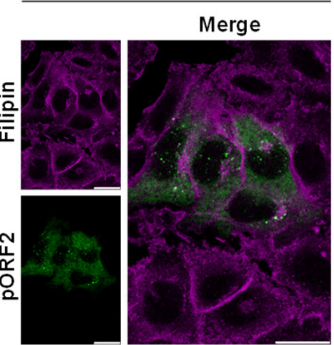

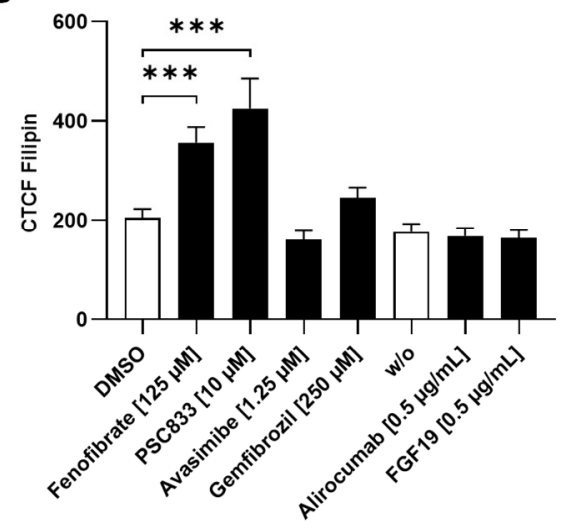

Figure 43. PSC833 and Fenofibrate efficiently increase intracellular cholesterol in A549 cells. (A) Representative CLSM images of Filipin III (magenta) and HEV pORF2 (green) of 48 h FGF19, Alirocumab, Gemfibrozil, Fenofibrate, Avasimibe or PSC833 treated, persistently HEV-infected A549 cells; scale bar $=20 \mu \mathrm{m}$. (B) Quantification of Filipin III signal intensity in (A) expressed as CTCF. w/o $=$ no treatment. ${ }^{* *} \mathrm{p}<0.001$, unpaired t-test with HolmSidak correction.

Analysing the fluorescent signal intensity of Filipin III revealed that neither Avasimibe nor Alirocumab or FGF19 impacted cellular cholesterol. However, both PSC833 and Fenofibrate led to a dot-like accumulation of cholesterol within treated cells being most pronounced for the latter. This in turn went along with an increased level of the lipid, proving efficient treatment (Figure 43A-B).

Both PSC833 and Fenofibrate rely on a certain pattern in cholesterol metabolism with respect to their mode of action. These are likely to be most comparable to an intact organism in hepatocytes. As this may differ in A549 cells, the effect of the drugcandidates was compared to Huh-7 cells. Therefore, both the pulmonary cell line as well as the hepatocyte cell line were treated with the drug-candidates for $48 \mathrm{~h}$ and CLSM analyses were applied to assess intracellular cholesterol levels (Figure 44). 
A

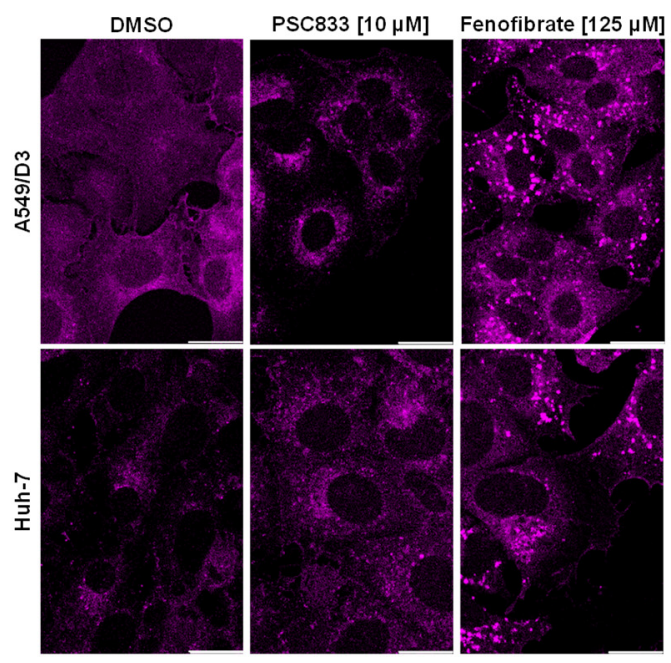

B

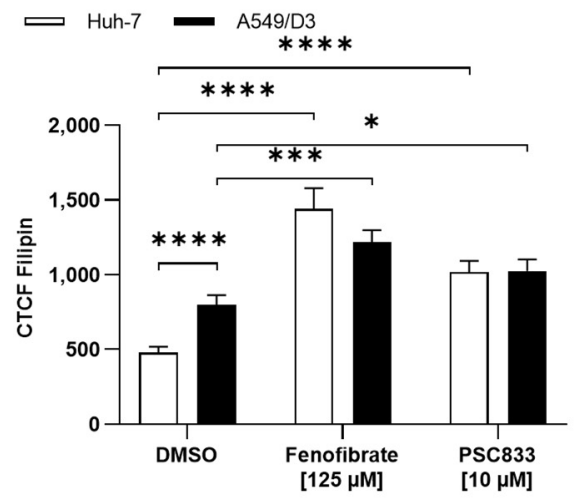

Figure 44. PSC833 and Fenofibrate act similarly on A549 cells as on Huh-7 cells. (A) Representative CLSM images of Filipin III (magenta) of $48 \mathrm{~h}$ Fenofibrate or PSC833 treated A549/D3 or Huh-7 cells; scale bar $=20 \mu \mathrm{m}$. (B) Quantification of Filipin III signal intensity in (A) expressed as CTCF. ${ }^{*} \mathrm{p}<0.05,{ }^{* * *} \mathrm{p}<0.001,{ }^{* * * *} \mathrm{p}<0.0001$, unpaired ttest with Holm-Sidak correction.

On the basis of a Filipin III stain, a certain difference in cellular cholesterol distribution became apparent between A549 and Huh-7 cells. In the first, the lipid was relatively equally distributed over the cell, while small accumulations could be detected. In Huh7 cells however, cholesterol accumulated in larger, dot-like structures (Figure 44A). Despite this, A549 cells were found to contain significantly more cholesterol as compared to Huh-7 cells (Figure 44B). As per the effect exerted by drug-candidates, both cells lines behave similarly. Here, Fenofibrate- as well as PSC833-treatment led to a significant increase in cellular cholesterol to a similar extent in both cell lines (Figure 44B). This effect was most pronounced upon Fenofibrate-treatment, which further showed a stronger induction of dot-like cholesterol accumulations.

Taken together, these data provide evidence for PSC833 and especially Fenofibrate being suitable drugs to increase intracellular cholesterol significantly. Furthermore, A549 cells again display to be an adequate system to study cholesterol-related effects in cell culture.

\subsection{Drug-induced elevation of intracellular cholesterol impairs viral release}

After assessing that a proportion of compounds increase intracellular cholesterol, drug-candidates were screened for their antiviral activity against HEV. Therefore, persistently HEV-infected A549 cells were treated with different concentrations of the compounds over 24-72 h. Subsequently, viral RNA release was monitored via qPCR (Figure 45). 
A

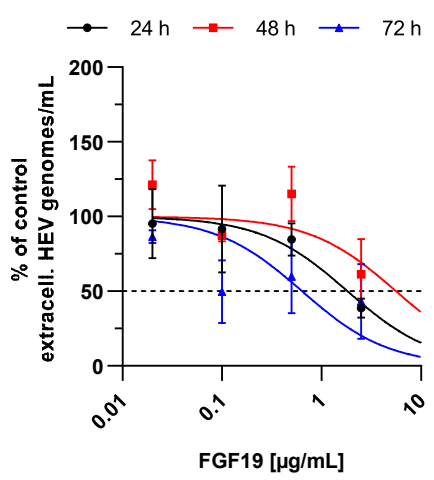

D

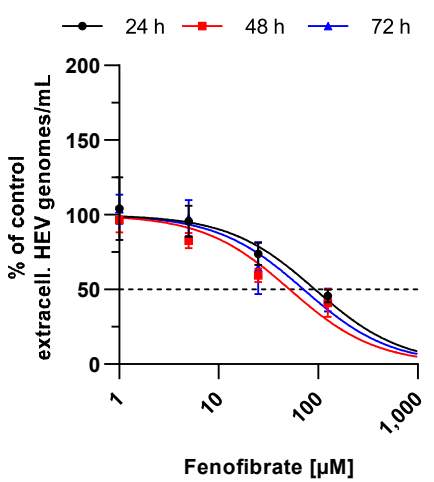

B

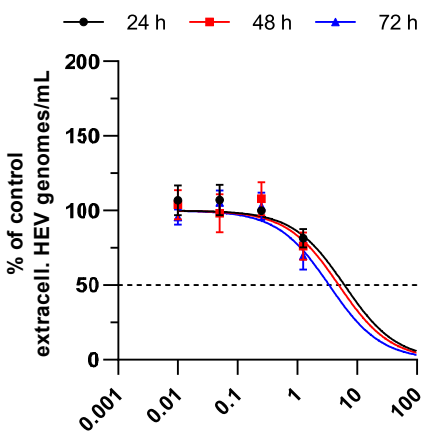

Avasimibe $[\mu \mathrm{M}]$

E

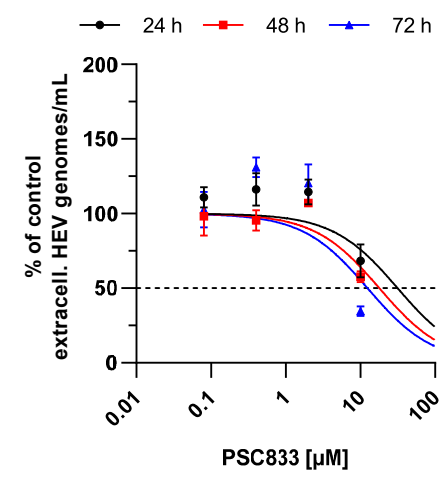

C

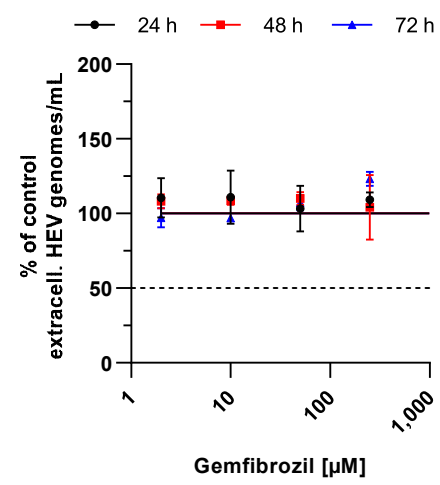

$\mathbf{F}$

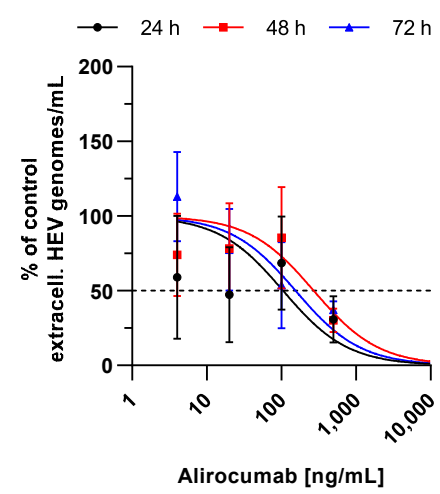

Figure 45. Cholesterol-modulating drugs efficiently inhibit viral release from persistently HEV-infected A549 cells. (A-F) Relative amount of viral genomes/mL released into culture supernatant of persistently HEV-infected A549 cells upon 24-72 h treatment with FGF19, Alirocumab, Gemfibrozil, Fenofibrate, Avasimibe or PSC833 as assessed RT-qPCR; values expressed as \% of control extracellular HEV genomes $/ \mathrm{mL}$ referred to the experimental control; curves fitted with an inhibitory dose-response model.

Assessment of the half-maximal effective concentration (EC50) within this screening unveiled that Gemfibrozil did not display antiviral activity. Both Avasimibe $\left(\mathrm{EC}_{50}=3.4 \mu \mathrm{M}\right.$ at $72 \mathrm{~h} ; 95 \% \mathrm{CI} 1.84$ to $\left.8.5 \mu \mathrm{M}\right)$ and FGF19 $\left(\mathrm{EC}_{50}=0.64 \mu \mathrm{g} / \mathrm{mL}\right.$ at $72 \mathrm{~h}$; $95 \%$ CI 0.08 to $4.27 \mu \mathrm{g} / \mathrm{mL}$ ) slightly reduced release of viral RNA (Figure 45A-C). A mild inhibition of viral RNA egress was found to be induced by Alirocumab $\left(E_{50}=155 \mathrm{ng} / \mathrm{mL}\right.$ at $72 \mathrm{~h} ; 95 \%$ CI 34.94 to $\left.810.4 \mathrm{ng} / \mathrm{mL}\right)$. Importantly, both Fenofibrate $\left(\mathrm{EC}_{50}=71.24 \mu \mathrm{M}\right.$ at $72 \mathrm{~h} ; 95 \%$ CI 34.52 to $\left.154.3 \mu \mathrm{M}\right)$ and PSC833 $\left(\mathrm{EC}_{50}=12.47 \mu \mathrm{M}\right.$ at $72 \mathrm{~h} ; 95 \% \mathrm{CI} 4.95$ to $48.0 \mu \mathrm{M}$ ) displayed a pronounced reductive effect exerted on the release of viral RNA (Figure 45D-F).

As three of the drug-candidates were proved to display an antiviral effect at least with respect to the release of HEV RNA, further analyses were implemented. Herein, all previously screened compounds were used to treat infected cells over the course of $72 \mathrm{~h}$. As released viral RNA does not directly correlate with released virions, the antiviral effect therefore was assessed via an EPDA. To correlate potential changes in buoyant-density of HEV particles to the increase in particle density found to be caused 
by increased intracellular cholesterol, isopycnic density-gradient centrifugations were performed for promising drug-candidates (Figure 46).

A

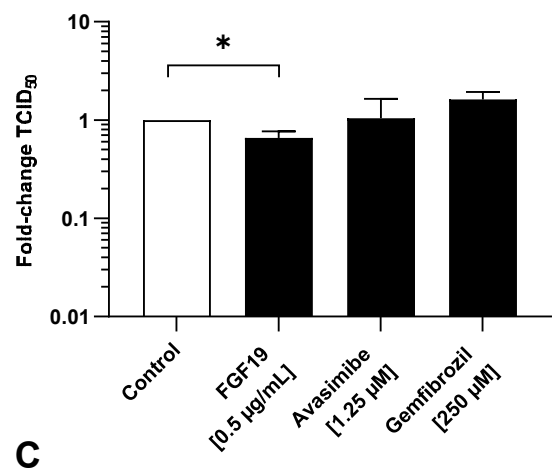

$\rightarrow$ nHEV

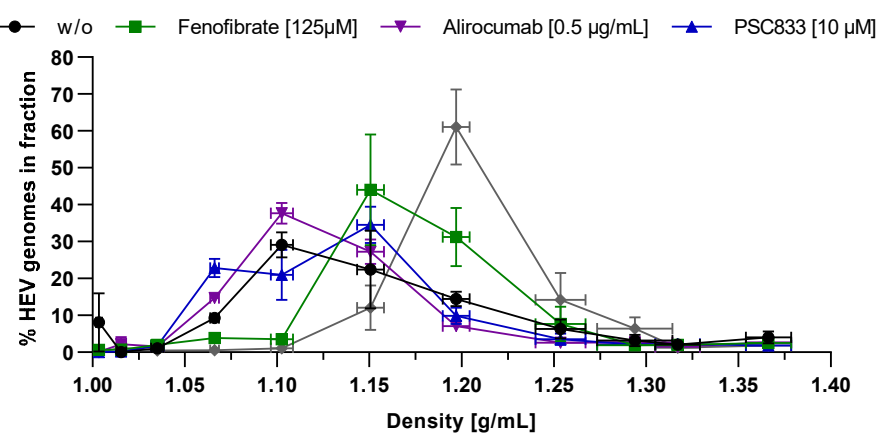

B

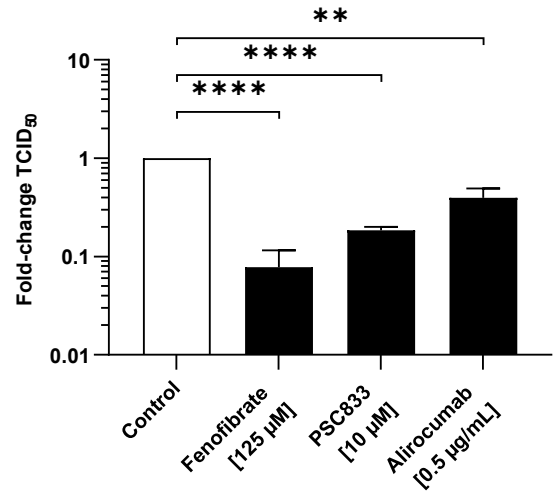

Figure 46. PSC833 and Fenofibrate prohibit efficient release of infectious viral particles from persistently HEVinfected A549 cells. (A-B) Relative change in number of infectious viral particles being released into cell culture supernatant of persistently HEV-infected A549 cells upon $72 \mathrm{~h}$ treatment with FGF19, Alirocumab, Gemfibrozil, Fenofibrate, Avasimibe or PSC833 as assessed in an EPDA; values referred experimental control. (C) Relative amount of HEV genomes found in density gradient fractions of HEV virions released by persistently HEV-infected A549 cells upon $72 \mathrm{~h}$ Alirocumab, Fenofibrate or PSC 833 treatment, values expressed as \% of genomes in the whole gradient being present in respective fraction; $\mathrm{nHEV}=$ taurocholate-treated virus stock. Control $=\mathrm{w} / \mathrm{o}$ for Alirocumab and FGF19 or DMSO for Avasimibe, Gemfibrozil, Fenofibrate and PSC833. ${ }^{*} \mathrm{p}<0.05,{ }^{* *} \mathrm{p}<0.01$, $* * * * p<0.0001$, unpaired t-test with Holm-Sidak correction.

Analysing the number of released HEV virions indeed implied that neither Avasimibe nor Gemfibrozil had the capacity to reduce virion release. Despite there being a significant reduction on virion release exerted by FGF19, this was only pronounced very weakly (Figure 46A). Similar to data displayed in Figure 45, Alirocumab did also affect virion release negatively and significantly. Alongside this, both PSC833 and Fenofibrate reduced HEV virion release tremendously (Figure 46B). These effects are in line with findings made for LDL- or 25-HC treated cells (Figure 38C), considered that elevated cellular cholesterol also triggered an increase in virion density (Figure 38E). This increase in density, yet not matching nHEV-density, could also be observed partially for PSC833-treated cells and especially for Fenofibrate-treated cells, the most efficient antiviral compound (Figure 46C). 
The above shown data demonstrate that cholesterol-modulating drugs do act antivirally against HEV. Specifically, PSC833 and Fenofibrate herein induce similar phenotypes as LDL and 25-HC with respect to virion density. This antiviral activity being most pronounced for these drugs does correlate particularly well with the most prominent effect exerted on intracellular cholesterol accumulation (Figure 44).

\subsection{Efficiently acting cholesterol-modulators induce lysosomal degradation of HEV}

As seen before, reductive effects on virion release may have different causes. Therefore, infected cells were subjected to treatments with the most promising drugcandidates over the course of $72 \mathrm{~h}$. To check for intracellular HEV pORF2 retention, reflecting an inhibition of viral release, or HEV pORF2 reduction, reflecting degradation, Western blot analyses were performed (Figure 47).

A

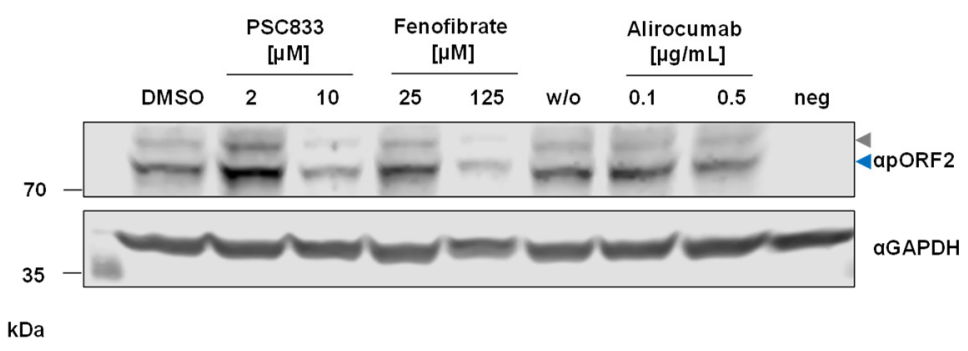

B

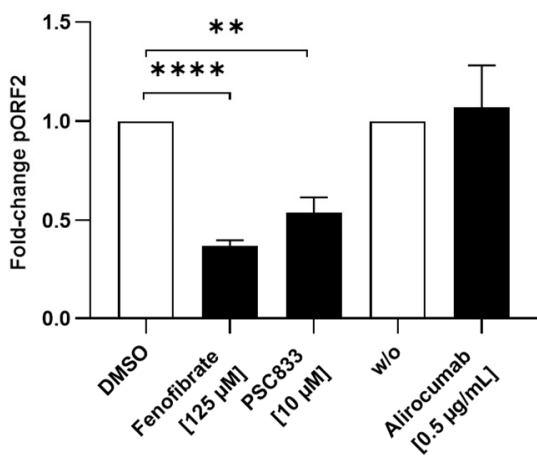

Figure 47. PSC833 and Fenofibrate reduce intracellular amount of HEV pORF2. (A) Representative Western blot of HEV pORF2 and GAPDH in total lysates of persistently HEV-infected A549 cells subjected to a $72 \mathrm{~h}$ treatment with PSC833, Fenofibrate or Alirocumab; blue arrow indicates unglycosylated HEV pORF2; grey arrow indicates glycosylated HEV pORF2. (B) Relative change in HEV pORF2 signal intensity in (A); values referred to experimental control. $\mathrm{w} / \mathrm{o}=$ no treatment. ${ }^{*} \mathrm{p}<0.05,{ }^{* * *} \mathrm{p}<0.001$, unpaired $\mathrm{t}$-test with Holm-Sidak correction.

Within this experimental setting, Alirocumab did not alter the intracellular amount of pORF2. Contrarily, both PSC833- and Fenofibrate-treatment led to a significant reduction in pORF2. In concordance with previous findings, Fenofibrate exerted the strongest reductive effect (Figure 47A-B).

As previous findings implied that HEV is incorporated into lysosomes once there are elevated cellular cholesterol levels at hand (Figure 39), this was addressed in a further experiment. Here, cells were treated with promising drug-candidates for $48 \mathrm{~h}$ to visualise the subcellular distribution of both HEV pORF2 and LAMP2 before the onset of the strong reduction in virion release. CLSM analyses were applied to validate Western blot findings and to address lysosomal localisation of pORF2 (Figure 48). 
A

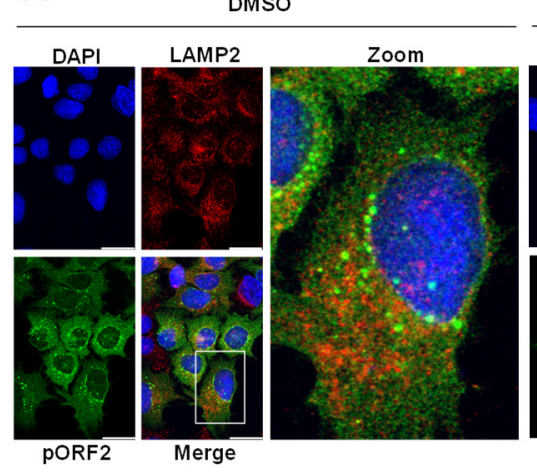

w/o

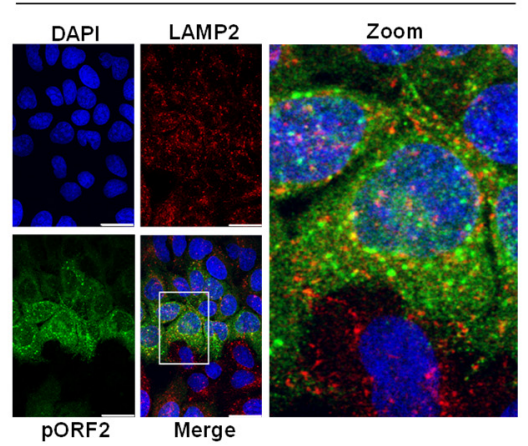

B
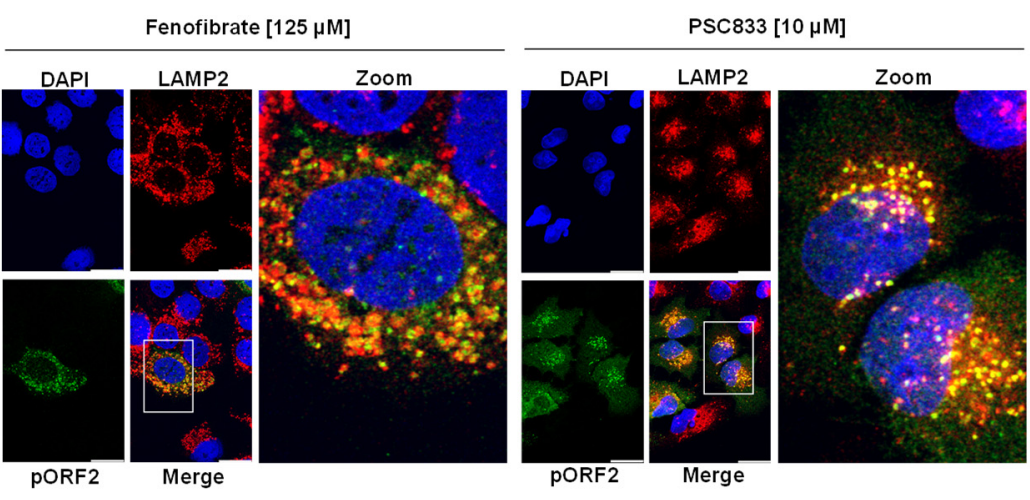

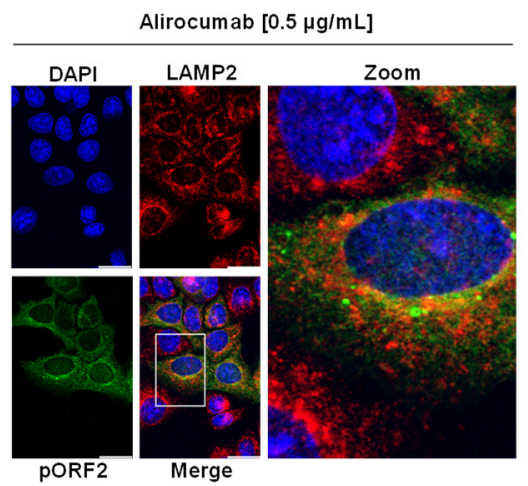

C
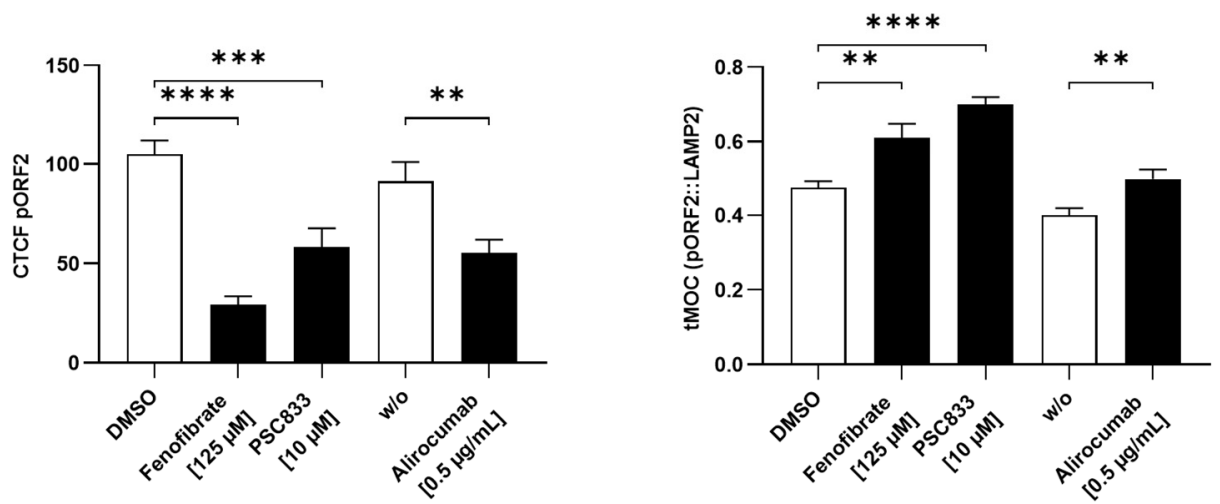

Figure 48. PSC833 and Fenofibrate efficiently reduce intracellular HEV pORF2 and induce its lysosomal localisation. (A) Representative CLSM images of DAPI (blue), HEV pORF2 (green) and LAMP2 (red) of persistently HEV-infected A549 cells under $48 \mathrm{~h}$ Fenofibrate, PSC833 or Alirocumab treatment; scale bar $=20 \mu \mathrm{m}$; zoom represents magnification from area in white rectangle. (B) Quantification of HEV pORF2 signal intensity in (A) expressed as CTCF. (C) Quantification of pixel co-localisation between HEV pORF2 and LAMP2 in (A) expressed as tMOC. $\mathrm{w} / \mathrm{o}=$ no treatment. ${ }^{* *} \mathrm{p}<0.01,{ }^{* * *} \mathrm{p}<0.001,{ }^{* * * *} \mathrm{p}<0.0001$, unpaired t-test with Holm-Sidak correction.

In single-cell analyses, a reduction of intracellular HEV pORF2 became apparent for the entirety of the tested compounds. In line with previous data, Fenofibrate displayed the strongest effect (Figure 48A-B). Just as demonstrated for cholesterol-loaded cells (Figure 39), pORF2 accumulated in dot-like structures upon treatment. This was most pronounced for treatment with Fenofibrate or PSC833 and to a small extent Alirocumab-treated cells (Figure 48A). These clustered structures of HEV pORF2 were 
found to co-localise with signals for the lysosomal marker LAMP2. In effect, the lysosomal co-localisation of pORF2 significantly increased upon treatment with Fenofibrate and PSC833 (Figure 48C).

Subsequently, Leupeptin was again used to inhibit lysosomal degradation. Infected cells subjected to Fenofibrate-, PSC833- or Alirocumab-treatment were then analysed for a potential abrogation of their antiviral effects (Figure 49).

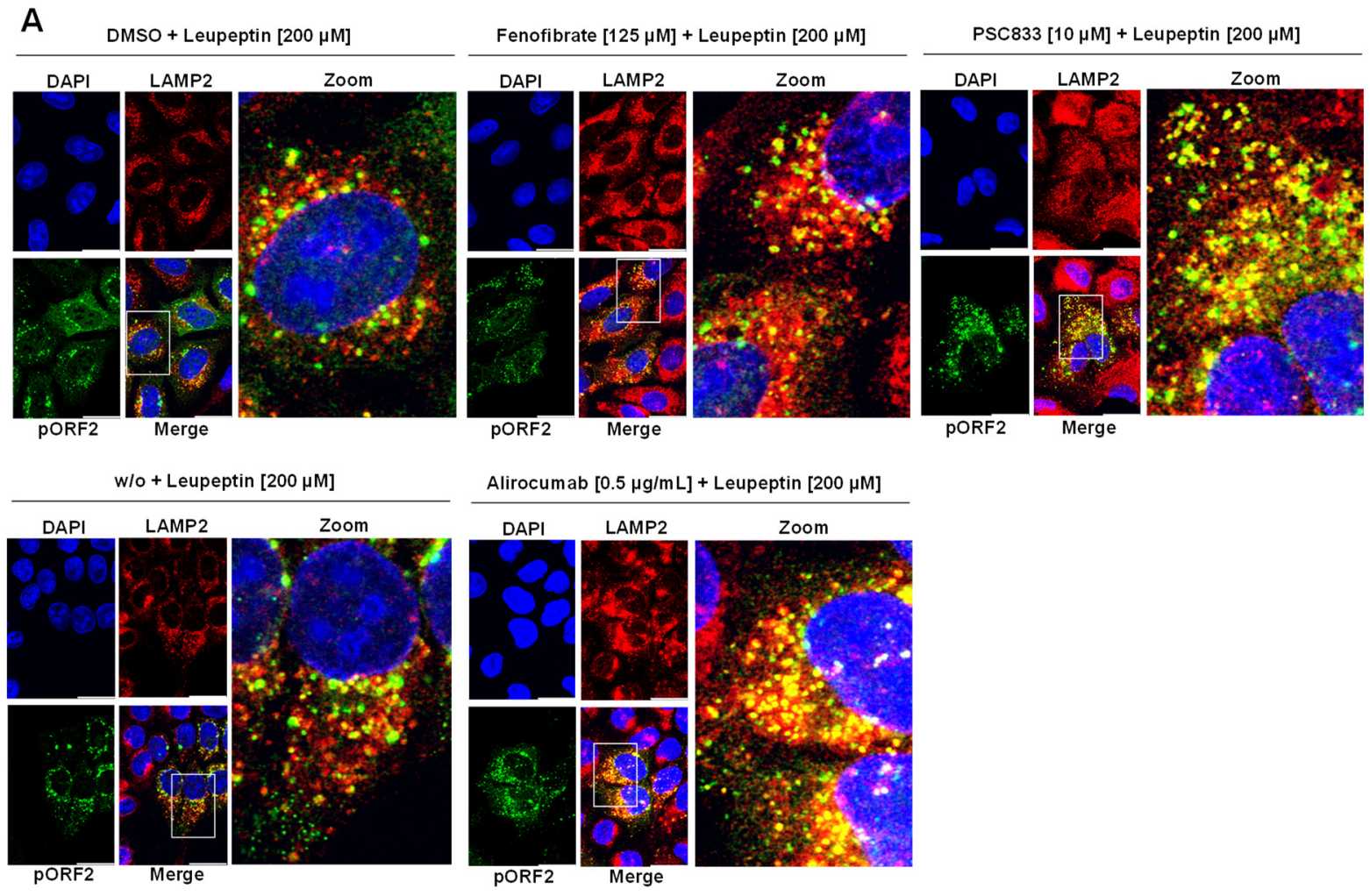

B

C
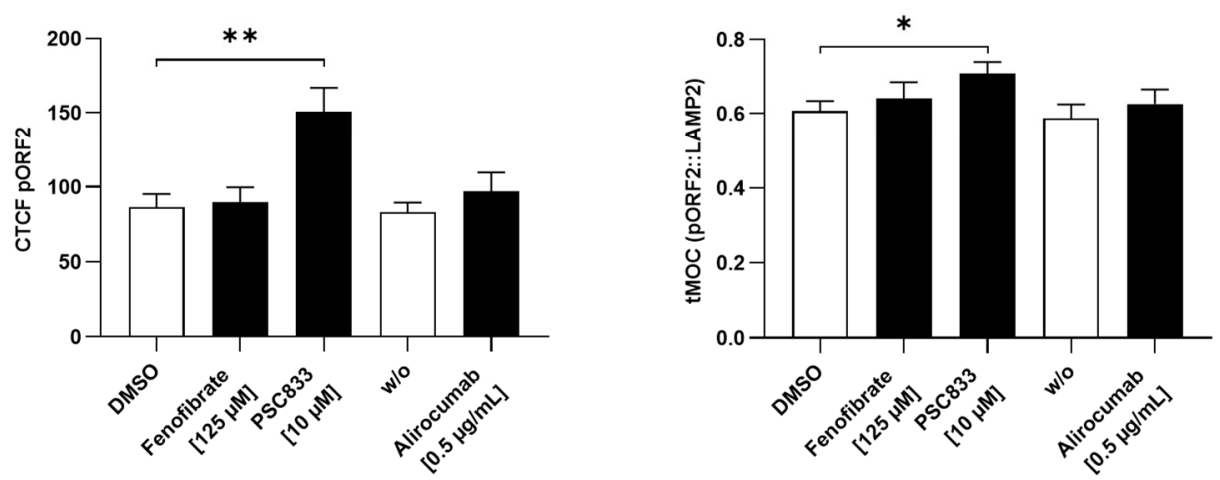

Figure 49. Blocking of lysosomal degradation rescues HEV pORF2 from drug-induced reduction. (A) Representative CLSM images of DAPI (blue), HEV pORF2 (green) and LAMP2 (red) of persistently HEV-infected A549 cells under $48 \mathrm{~h}$ Fenofibrate, PSC833 or Alirocumab treatment without or with $200 \mu \mathrm{M}$ Leupeptin treatment over $24 \mathrm{~h}$; scale bar $=20 \mu \mathrm{m}$; zoom represents magnification from area in white rectangle. (B) Quantification of HEV pORF2 signal intensity in (A) expressed as CTCF. (C) Quantification of pixel co-localisation between HEV pORF2 and LAMP2 in (A) expressed as tMOC. w/o $=$ no treatment. ${ }^{*} \mathrm{p}<0.05,{ }^{* *} \mathrm{p}<0.01$, unpaired $\mathrm{t}$-test with Holm-Sidak correction. 
All of the treated cells displayed a rather equally intense HEV pORF2 signal. Solely PSC833 was found to increase HEV pORF2 levels beyond the experimental control (Figure 49A-B). This trend was also visible when analysing the co-localisation between HEV pORF2 and LAMP2. Here, all treatments displayed a consistent lysosomal localisation of pORF2, yet only PSC833-treatment yielded a slight increase (Figure 49C). Most importantly however, co-administration of Leupeptin did indeed abolish the reductive effects exerted on pORF2 by the cholesterol-modulating drugs.

Taking the above shown data into account, a regulatory role of cholesterol with respect to the life cycle of HEV is evident. An HEV-infection alters cellular gene-expression and yields reduced cellular cholesterol. This reduction in cholesterol is beneficial for viral release in vitro and in vivo. On the contrary, elevating cellular cholesterol induces lysosomal degradation of the virus and alters its quasi-envelope. This can efficiently be induced via both IFN-application and cholesterol-modulating drugs such as Fenofibrate or PSC833. 


\section{Discussion}

In viral infections, the pathogen relies on host-factors and -machineries for efficient replication and spread of progeny. One distinct structure being hijacked by HEV is represented by the endosomal system, more precisely MVBs, which is used for quasienvelopment and subsequent virion release $[34,103,106]$. These endosomal structures are strongly intertwined with the lysosomal system, together forming one continuum of gradually maturating, vesicular structures [209]. This correlation potentially makes HEV particularly vulnerable to two major aspects: (i) innate immunity affecting endolysosomal structures [401,402] and (ii) cholesterol as essential lipid for MVB morphogenesis [236,403]. Analysing both determinants in more detail was subject to this study.

Innate sensing in infected cells, regardless of associated PRRs, generally elicits an IFN type I induction via transcriptional regulation [404]. This effect is visible when comparing basal ISRE-activity in persistently HEV-infected A549 cells with uninfected cells (Figure 20A). Interestingly, an HEV-infection alone does not induce GAS-activity (Figure 20B), which may be explained by various secondary processes being involved in transcriptional activation of IFN $\gamma$-expression, therefore prolonging the time until an induction is visible [405]. As consequence, an HEV-related augmentation in the proximal GBP1-promoter activity (Figure 20C), which contains both ISRE and GAS $[172,406]$, may be mainly driven by ISRE. Exogenous application of IFN $\alpha,-\beta$ or $-\gamma$ proved to be successful in uninfected cells with respect to GBP1-promoter induction, yet HEV-infected cells reacted differently (Figure 20C). A lack of substantial promoteractivation upon IFN $\alpha$ or $-\beta$-stimulation in the latter could be caused by HEV proteins interfering with IFNR downstream signalling [163,164,166,167,407]. These differential promoter-based effects may be interesting for potential immune-evasion strategies, as seen for other viral infections [408]. In effect, however, GBP1 mRNA was induced by all tested interferons (Figure 20D) displaying most pronounced effects upon IFN $\gamma$ stimulation. In turn, the type II IFN exerts the strongest antiviral effect on the basis of intracellular viral transcripts (Figure 20E). Interestingly, when focusing on basal levels of GBP1 mRNA, an early infection with HEV gradually reduces GBP1-encoding transcripts over time (Figure 21C). The same holds true for a long-term, persistent HEV-infection (Figure 23B). This reduction in GBP1 mRNA may be explained by HEV making use of an immune-evasion strategy counteracting the ISG, possibly through activation of the oligoadenylate-synthetase (OAS)-latent RNase (RNase L) [409]. Even more surprising is the fact that GBP1 protein levels appear to be significantly elevated, as also seen for an infection with e.g. Dengue virus (DENV) [193], as early as 9 days post infection (Figure 21D-G and Figure 22) up to a persistent infection (Figure 23CF), when compared to uninfected cells. This discrepancy between transcript and 
protein amount is explained by infection-related changes in GBP1 protein half-life. Evidently, the viral infection slows GBP1 turnover, yielding more protein being present in infected cells despite there being less mRNA (Figure 24). One hypothesis for this stabilising effect being exerted by HEV on GBP1 could be of autophagosomal nature. Both GBP1 and HEV are involved in autophagic processes [105,410,411]. Further, p62, an autophagosomal adaptor [412], displays a slightly elevated half-life in HEV-infected cells (Figure 24C). HEV-mediated effects on (auto-)lysosomal degradation could therefore also affect GBP1 co-degradation, as it is present on structures targeted for destruction [197,413]. In essence, this cross-talk of GBP1, HEV and the lysosomal system is of central interest for this study.

An abundant presence of GBP1, achieved via an overexpression, reduces virion release dramatically (Figure 25C). Alongside this, significantly reduced levels of intracellular HEV pORF2 can be found in GBP1wt-overexpressing cells (Figure 26A-B). Notably, pORF2 in the latter is mainly found in dot-like structures representing lysosomes (Figure 26A, D and E). This gives rise to a GBP1-induced increase in lysosomal localisation of HEV pORF2 (Figure 26C). The presence of viral structures within these degradative structures ultimately leads to their destruction. Clear evidence for this is found once the related degradation is prohibited by the application of Leupeptin [414], where intracellular pORF2 levels were rescued from the GBP1-induced degradation (Figure 26B). Responsible for the incorporation of HEV into lysosomes could be two different functions of GBP1: (i) enhancement of membrane fission/fusion events exerted via its dynamin-activity $[185,415,416]$ or (ii) fulfilling an adaptor role for cargo being targeted for degradation $[417,418]$. This was addressed via overexpression of either GBP1-R48A, which is GTPase-activity deficient [183], or GBP1-S73A, which cannot form homodimers and therefore is less prominently found on membranes [175]. Overexpression of the first completely retains antiviral effects against HEV, which stands in contrast to previous findings for other RNA viruses [191,192]. This effect includes the reduction in pORF2, the causative lysosomal localisation with subsequent degradation and the resulting reduction in virion release (Figure 27). Contrarily, introduction of an S73A mutation abolishes the before mentioned antiviral effects exerted by GBP1 inclusive of lysosomal pORF2 localisation (Figure 27). Hence, GBP1 does not require the dynamin function, which is dependent on functional GTPhydrolysis, to act efficiently against $\mathrm{HEV}$, but it requires homodimerisation. In turn, this suggests GBP1 representing a factor being majorly involved in targeting HEV to lysosomal degradation.

As GBP1 is only one within a variety of different ISGs, overexpression alone does not suffice to prove its relevancy in innate immunity targeting HEV. Thus, the IFN $\gamma-$ mediated antiviral effects against the virus were assessed in cells where GBP1 was silenced. The type II IFN alone reduces both intracellular pORF2 and very drastically 
virion release (Figure 28). This proves to be just as pronounced as a GBP1overexpression. Importantly, once the ISG is silenced, this effect is reverted, rendering IFN $\gamma$ to be strikingly less efficient in acting antivirally (Figure 28C and D). Reductions in GBP1 levels alone are described to influence a set of intracellular mechanisms $[193,410,419]$. Surely, it cannot be ruled out that one of these may be involved in providing a certain IFN $\gamma$-resistance with respect to HEV. However, the IFN-induced lysosomal localisation and degradation of HEV pORF2 is significantly less pronounced in GBP1-silenced cells (Figure 29). In fact, this interference with GBP1expression within IFN $\gamma$-stimulation clearly prohibits HEV from being incorporated into lysosomal structures (Figure 30), therefore rescuing virions from being degraded.

In summary, an infection with HEV induces intracellular protein levels of GBP1, an IFN $\gamma$-induced GTPase. This is achieved via both triggering ISRE-dependent geneexpression as well as prolonging the protein's half-life. On the other hand, both a GBP1-overexpression as well as IFN $\gamma$-stimulation, induce a prominent antiviral effect against HEV. This in turn, is exerted via lysosomal degradation. Without a sufficient amount of GBP1, lysosomal incorporation of HEV can no longer take place as efficiently. Hence, GBP1, acting through homodimerisation, must represent an essential factor being involved in efficient IFN $\gamma$-induced lysosomal degradation of HEV.

Apart from the above assessed mechanisms, IFNs also bear the capacity to modulate intracellular cholesterol $[420,421]$. Besides this, downstream effects further allow for modulation of the lysosomal network [402]. This goes in line with data presented in this study, where IFN $\alpha,-\beta$ and $-\gamma$ evoke changes in gene-expression of cholesterol related genes (Figure 31). In essence, these changes elicit an increase in intracellular cholesterol (Figure 32A-B), which is again most pronounced upon IFN $\gamma$-stimulation. Most importantly, these increases in intracellular cholesterol mainly contribute to cholesterol-loading of lysosomes (Figure 32C), which is consistent with previously published observations [422]. Notably, cross-regulatory events were described for cholesterol-removal transcription factors and the IFN system. Here, LXR can induce IFN $\gamma$-production [423], yet the type II IFN in turn reduces LXR-activity [424]. Similarly, FXR may induce IFN $\gamma$ [425], yet its activity is suppressed in the presence of it [426]. Opposing to this, $\mathrm{RXR} \alpha$ seems to prohibit efficient type I IFN signalling [427] and type II IFN production [428]. In effect however, studies suggest that IFNs mainly trigger prevention of sufficient FXR- and LXR-dependent cholesterol efflux, thereby possibly increasing cellular cholesterol [422,429-431]. As HEV induces innate immunity and the latter triggers changes in intracellular cholesterol, influences of a viral infection on the lipid were assessed. An infection with the Hepevirus does indeed strongly dysregulates gene-expression of a variety of different cholesterol-related genes (Figure 33A-B). Surprisingly, this does not lead to cellular cholesterol elevation, 
but to an overall reduction in cholesterol levels (Figure 33C-D), indicating that the virus could prohibit effects of innate immunity on cellular cholesterol. For these purposes, the virus potentially makes use of oxysterol-mediated efflux. Both $\mathrm{CH} 25 \mathrm{H}$ and ABCA1 expression is increased in infected cells. The protein encoded by the first catalyses cholesterol-hydroxylation on position C-25, yielding an oxysterol, namely 25HC. This lipid induces ABCA1-expression [432], which may be a reason why these genes are also upregulated upon an HEV-infection (Figure 33B). Further, it is substrate to ABCA1, which transfers the oxysterol to apoA-I, ultimately detoxifying cells from cholesterol by release of HDL [433]. Connecting these with the lysosomal cholesterolexport function of NPC1/2, based on previous studies [332,434,435], could provide important insights into a potential regulation by HEV. An additional answer as to how HEV is capable of reducing cellular cholesterol could be found by an induction of PCSK9-expression (Figure 33B). The protein encoded by this gene targets LDL-bound LDLR to lysosomal degradation instead of recycling [436]. This indicates that an HEVinfection could prohibit efficient LDL uptake. Importantly, the above described cholesterol-reducing effects are not limited to cell culture, as analyses of lipid concentrations in HEV-infected patients demonstrate. Upon infection, LDLcholesterol, triglycerides as well as total cholesterol are significantly reduced in renal transplant patients (Figure 34). Although changes in lipid profiles of patients suffering from viral infection is not unknown for various viruses [437-439], a relationship to HEV has never been drawn before. Moreover, in vitro as well as in vivo data imply that HEV may reduce cholesterol to create favourable conditions for the viral life cycle.

With respect to this hypothesis, infected cells were subjected to exogenous supplementation of LDL or 25-HC, increasing intracellular cholesterol, or Simvastatin, reducing intracellular cholesterol (Figure 36). All of these treatments contributed to diminished intracellular HEV pORF2 levels (Figure 37). However, this decrease cannot be deduced as simple antiviral effect. In fact, a Simvastatin-mediated reduction in cellular cholesterol and pORF2 is correlated with enhanced virion release (Figure 38C). This holds true for statin-mediated effects in chronically HEV-infected renal transplant patients. Subjection of the latter to statins, namely Pravastatin, Fluvastatin, Atorvastatin or Simvastatin, similarly leads to an elevation of the viral load in sera (Figure 38D). Again, this is in contrast to a variety of other viruses which were shown to be inhibited by application of Simvastatin [440-443]. Coherently, this finding is of utmost importance for clinical assessment of treatments in HEV-infected patients, as statin-use could be counter indicated. Given that this provides further evidence for the hypothesis of reduced cholesterol being beneficial for HEV, increased cellular cholesterol should be non-beneficial. Indeed, the LDL- and 25-HC-mediated reduction in pORF2 is accompanied by a reduction in the number of released virions (Figure 38C). These findings are in line with previous studies suggesting an antiviral role of 
25-HC [444-447]. Apart from the reduction in viral release, the properties of HEV virions change, as their specific buoyant-density is increased upon supplementation of cholesterol (Figure 38E). This however, does not lead to a loss of the quasi-envelope, but may reflect changes in how the virions are released and which composition the viral envelope comprises. An answer as to how this cholesterol-mediated reduction in pORF2 and virion release is caused, is provided in CLSM analyses. Once additional cholesterol is supplied to infected cells, pORF2 accumulates in dot-like structures (Figure 39A), which is reminiscent of effects detected in innate immunity. Just as under IFN $\gamma$-treatment, these dots are identified as lysosomes, which therefore yields an elevated lysosomal localisation of HEV pORF2. Appositely, this subcellular localisation is reduced in Simvastatin-treated cells (Figure 39C). In conclusion, a cholesterol-dependent incorporation of HEV into lysosomes is evident. Thereafter, the virus is degraded, which is supported by application of Leupeptin restoring pORF2 amounts in LDL- and 25-HC-treated cells, yet not in Simvastatin-treated cells (Figure 40). Part of the underlying mechanisms could be cholesterol-RILP/ORP1L-mediated endosomal trafficking towards lysosomes [448] in combination with a coupled, targeted transport [292]. Understanding this in more detail will help to newly characterise the fate of HEV within the endosomal system, as previous studies suggested e.g. HIV to modulate the RILP-ORP1L-axis [449]. Further, this relationship unravels a novel strategy for an antiviral treatment against HEV: using cholesterolmodulating drugs as a tool to induce cholesterol-dependent, lysosomal degradation of the virus.

In order to follow this aim, drugs efficiently increasing cellular cholesterol are needed. Within the drug-screening of non-cytotoxic and non-cytostatic concentrations (Figure 41 and Figure 42), both the PPAR $\alpha$-agonist Fenofibrate as well as the p-glycoprotein inhibitor PSC833 represent promising candidates. These elevate intracellular cholesterol levels significantly and lead to accumulations of the lipid (Figure 43). Furthermore, they display similar effects in A549 cells and in Huh-7 cells, indicating intact regulatory functions in the used cell culture model (Figure 44). All of the tested drug-candidates inhibit viral RNA release to a certain extent, with the exception of Gemfibrozil (Figure 45). This is rather surprising indeed, as Fenofibrate, being part of the same drug-family, exerts a very strong inhibitory effect on HEV. Although both represent Fibrates, they differ with respect to their pharmacokinetic properties $[450,451]$, which could explain the discrepancy in observed effects. The inhibitory capacity of drug-candidates was further assessed with regards to release of viral RNA. While all substances except for Gemfibrozil displayed the capacity to inhibit this process (Figure 45), a different picture arose when analysing the number of released infectious virions. Herein, it becomes apparent that neither the CYP7A1-suppressor FGF19, the ACAT-inhibitor Avasimibe nor Gemfibrozil display relevant antiviral 
effects (Figure 46A). The discrepancy between egress of viral RNA and virions may be due to impairment of a general release of intracellular components via extracellular vesicles. Importantly however, drugs not affecting virion release similarly do not exhibit cholesterol-modulating effects in A549 cells (Figure 36). While this may explain these to fail acting antivirally, the reason why cholesterol is unaffected remains elusive. An explanation for this could be the low tissue-expression of CYP7A1 in lung cells, where CYP7B1 is predominantly found [452]. As an effect, a further reduction would not affect the overall amount of present protein, therefore rendering FGF19 to be inefficient. A similar reason may explain lacking effects of Avasimibe. As this compound inhibits cholesterol-esterification to fatty acids via ACAT [453] and subsequent storage, an active storage needs to be present in the first place. Although A549 cells display overall increased cellular cholesterol as compared to Huh-7 cells, dot-like cholesterol-containing structures are sparsely populated in the pulmonary cells (Figure 44). Importantly, these often represent lipid droplets, where esterified cholesterol is stored [454]. Hence, if no substantial cholesterol storage occurs in A549 cells, an inhibition of this process to make free cholesterol available would fail. Opposing to these compounds, the PSCK9-inhibitor Alirocumab, PSC833 and Fenofibrate inhibit virion release drastically, in ascending order (Figure 46B). A correlation between their capacities to increase cellular cholesterol (Figure 43) is evidence to this effect really being exerted by influencing the lipid. As visible in these data, Alirocumab does not lead to a visible increase in cellular cholesterol. While there certainly may be an effect, it is beyond the level being detectible in the experimental approach set forth. In vivo, however, this inhibitor could prove useful, as there is more LDL present as compared to DMEM supplemented with FCS. This in turn could enhance the antibody's mode of action. A further aspect highlighting data integrity is the effect both PSC833 and Fenofibrate exert on virion density. Only for these, an increase in buoyant-density of eHEV can be detected (Figure 46C), which is in line with phenotypes observed for exogenous supplementation of cholesterol (Figure 38E). Concordantly, treatment of infected cells with Alirocumab, PSC833 or Fenofibrate decreases intracellular HEV pORF2 (Figure 47 and Figure 48A-B). Accompanying this reduction again, is a dot-like clustering of the viral protein, which is tracked down to an enhanced co-localisation with lysosomes (Figure 48C). As a final proof of an achieved induction of cholesterol-dependent lysosomal degradation upon treatment, Leupeptin was applied for similar reasons as above. This blockage of lysosomal degradation abrogates reductive effects being exerted by the drug-candidates (Figure $49)$, fully supporting the underlying hypothesis. This mechanism has the potential of being of universal use, as e.g. Fenofibrate displays antiviral effects also on other viruses [455]. 
In summa, the above presented data indicates that a second effector of IFN $\gamma$, apart from GBP1, may be represented by cholesterol. The lipid accumulates in lysosomes, thereby increasing cholesterol-loads in the endolysosomal system. Opposing to this, HEV efficiently reduces cellular cholesterol via dysregulation of related geneexpression, thereby favouring virion release in vitro and in vivo. In this context, administration of Simvastatin to HEV-infected patients may be counter indicated. More importantly however, the cholesterol-loading of the endolysosomal system results in HEV degradation via the degradative structures. These findings call attention to a variety of secondary effects being involved in the process. Cholesterol cannot be solely reduced to affecting membrane fluidity or metabolic processes, yet its anabolism also comprises substrates for prenylation. Thus, by affecting cholesterollevels, regulatory processes may evoke manifold changes in cellular processes. On the one hand, cholesterol-related metabolic changes (Figure 36) certainly affect peroxisomes [456] and mitochondria [457]. Consecutively, changes in cellular reactive oxygen species may result, which were described to play a role in the life cycle of e.g. the Hepatitis C virus [458]. On the other hand, alterations in cellular cholesterol also affects post-translational protein lipidation [459]. One primary target of protein prenylation are small GTPases, which orchestrate the flux in the endosomal system [460]. As these represent important gatekeepers in the fate of endosomal vesicles and were described to fulfil major roles in the life cycle of a variety of different viruses [461-464], HEV could certainly be affected just as well. Furthermore, farnesylation is of central importance when it comes to GBP1 oligomerisation [185]. This in turn couples the antiviral factor directly to cholesterol metabolic processes. In how far GBP1 on the other hand affects cellular cholesterol remains elusive, however previous studies have linked it to atherosclerosis [465]. One further hint is found in the presented study, where GBP1 silencing seemingly reduces LAMP2 dot-formation under IFN $\gamma$-stimulation (Figure 30). Hence, a more detailed study analysing the involvement of GBP1 in lysosomal cholesterol accumulation would prove informative.

Phenotypically and effectively, this cholesterol-dependency of HEV can be compromised by employing cholesterol-modulators such as PSC833 or Fenofibrate. Assuming that the lipid modulation of IFN $\gamma$ represents one of the fundaments of a PEG-IFN-treatment in HEV-infected patients, a targeted approach would definitely provide a safer treatment option. Despite there being clear evidence that the aforementioned drugs represent strong antivirals, some concerns must be addressed. One of these is that PSC833 is a p-glycoprotein inhibitor. With this, it does not exclusively target ABCA1, but it inhibits a variety of ABC-transporters [466]. This circumstance could therefore lead to side effects targeting co-treatments with other medication. While this may be useful for a co-treatment of patients with Ribavirin, which potentially is thereafter retained more prominently at sites of action, it could 
render other drugs to exceed their optimised dose due to retention in cells or make them inefficient. Furthermore, PSC833 is designed to support chemotherapy via reduction of multidrug resistance [467], the use of A549 cells, a cancer cell line, could prove to be a problematic system. As far as Fenofibrate is concerned, the application in morbid patients should be carefully assessed, due to reported side-effects affecting the liver. However, the use of Bezafibrate, residing within the group of fibrates as well, in primary biliary cholangitis (PBC) argues for a rather safe use of these drugs even in patients suffering from hepatic diseases [468]. In essence, the presented study opens to door for a drug-repurposing approach focusing on Fenofibrate as an agent triggering cholesterol-dependent lysosomal degradation of HEV.

As final remarks and conclusions, it can be stated that IFNs fulfil a dual role in restricting HEV by affecting cellular cholesterol, but more importantly inducing the GTPase GBP1. In this study, the latter is found to be one of the major effector molecules induced by IFN $\gamma$. It exerts its effect via targeting HEV to the lysosomal compartment where degradation takes place. For this process, not GTPase-activity, but homodimerisation is of fundamental importance [469]. In future studies, this process will be further dissected to pinpoint co-factors and upstream structures being involved in the GBP1-related targeting of HEV to lysosomes. Ultimately, the goal hereof is the identification of causative factors that can be modulated by drugs and therefore be applied to clinical use. This major aim was successfully achieved for the second part of this study. HEV was identified to be efficiently degraded lysosomally in a cholesterol-dependent manner. The virus itself induces measures to counteract this phenomenon. However, the cholesterol-dependent lysosomal degradation can be successfully induced by applying drugs, most promisingly via Fenofibrate [470]. One important aspect of having identified this is that Fenofibrate is already clinically approved. Therefore, upcoming animal-studies will show whether this treatment is as efficient in vivo as it is in vitro. Success in this would pave the way for the introduction of a novel antiviral being cheap in production and free of risks of inducing viral mutations conferring drug-resistance. 


\section{Summary}

With roughly 20 Million newly registered cases per year, HEV represents the major agent causing an acute viral hepatitis worldwide. It is responsible for over 22,000 annual fatalities and poses severe risks especially for pregnant women and immunosuppressed patients, where fulminant pathogeneses and chronification occur most frequently, respectively. As an infection can be transmitted via various routes, namely contaminated food, water and blood-products, manifold populations are at risk. Despite this, only two treatment options are available: (i) Ribavirin and (ii) PEGIFN. Both carry high risks of severe adverse effects, whereas Ribavirin even induces drug-resistance in the viral polymerase. Thus, new insights into how HEV modulates host-cells and which factors are crucial to viral egress are urgently needed to tailor novel antivirals.

An HEV-infection is mainly sensed within an infected cell via TLRs and RLRs with respect to innate immunity. This in turn triggers IFN-production and subsequent cellular IFN-response. A major ISG induced by IFN $\gamma$, which evidently represses HEV most efficiently, is represented by GBP1. In this study, it has been identified that this ISG is induced upon an HEV-infection. This is achieved via the promoter element ISRE and induction of the proximal GBP1-promoter. Interestingly, while GBP1-secific mRNA levels are contradictorily reduced in infected cells, the protein amount remains elevated. This discrepancy is based on an HEV-mediated stabilisation of GBP1 protein half-life, effectively inducing the ISG as an IFN-effector. An abundance of GBP1 was therefore analysed via overexpression in persistently HEV-infected cells with respect to whether or not antiviral effects are in place. Here, GBP1 efficiently reduces both intracellular HEV pORF2 levels, as well as the number of released virions. It does so by inducing lysosomal incorporation of viral particles, which ultimately leads to their destruction within the degradative structures. As GBP1 represents a dynamin-like GTPase, underlying mechanisms were investigated by overexpression of GBP1mutants. The mutation R48A leads to GTPase-deficiency, thus abrogating dynaminfunction and GTP-hydrolysis, whereas the mutation S73A is detrimental for GBP1 homodimerisation, rendering reduced farnesylation and subsequent membraneassociation. While the first retains its antiviral effect, the latter loses its capacity to induce HEV-degradation. This in turn means that GBP1 homodimerisation is required for an active antiviral effect, implying that GBP1 may serve as adaptor-molecule for lysosomal incorporation. The relevance of GBP1 within IFN $\gamma$-induced antiviral effects was analysed via siRNA-mediated silencing under IFN-stimulation. Herein, IFN $\gamma$ induces a similar lysosomal incorporation of HEV followed by its destruction and a dramatic drop in viral egress. On the other hand, once GBP1 is silenced, this effect is far less pronounced, rendering IFN $\gamma$ to be inefficient in fulfilling antiviral effects. This 
led to the identification of GBP1 as a major restriction factor against HEV, which does so via homodimerisation-dependent induction of lysosomal degradation.

A phenotype visible under IFN-stimulation is an accumulation of cholesterol within infected cells. As the lipid represents an essential modulator of endosomal maturation, trafficking and functionality, it may directly affect HEV. Reason for this is HEV using MVBs for viral egress, which require an intact cholesterol-homeostasis to achieve e.g. ESCRT-dependent ILV-formation and trafficking to the PM. Thus, cellular cholesterol levels and transcriptional footprints were analysed in HEV-infected cells. The virus herein modulates gene-expression of central factors within the cholesterolhomeostasis, ultimately leading to decreased intracellular cholesterol levels. A similar situation is found in HEV-infected patients, where serum-lipid concentrations diminished over the course of infection. This implies that reduced cholesterol levels are in favour of HEV. To prove this, cellular cholesterol was modulated, where an inhibition of cholesterol biosynthesis via Simvastatin induces viral release significantly. Importantly, this is also the case in chronically HEV-infected patients, where statin-treatment results in elevated viral serum titres, implying a counterindication. On the contrary, elevation of cellular cholesterol levels in vitro via supplementation of LDL or 25-HC, results in diminishing amounts of both intracellular pORF2 and viral egress. Just as for an IFN-stimulation, causative for this HEV-inhibition is lysosomal degradation. Under cholesterol-elevation, virions are incorporated into the structures and subsequently degraded. Whether exploitation of this mechanisms serves antiviral purposes was addressed in screening different cholesterol-modulators for their activity to repress HEV. While Avasimibe, Gemfibrozil, FGF19 and Alirocumab only display no or minor effects against HEV, the p-glycoprotein inhibitor PSC833 as well as the PPAR $\alpha$-agonist Fenofibrate prove to be efficient. In line with the underlying hypothesis, both compounds lead to a drastic increase in cellular cholesterol accumulation within vesicular structures. Effectively, this dramatically increases lysosomal localisation of $\mathrm{HEV}$ and results in viriondestruction. This in turn evokes significant reductions in viral egress, providing strong evidence for the compounds being promising antivirals against HEV.

In summary, this study elucidated the essential functions of GBP1 with respect to its restrictive effect against HEV. Further, the ISG was identified as crucial host-factor for the IFN $\gamma$-response against the virus in a lysosome-dependent manner. On the other hand, the presented work suggests that HEV requires low cellular cholesterol levels for efficient viral release and maintaining the viral life cycle. Disruption of the lipid homeostasis, resulting in elevated cholesterol levels, is detrimental for viral release, as lysosomal degradation is induced. This led to successful discovery of a novel antiviral efficiently inducing this cholesterol-dependent effect: Fenofibrate. 


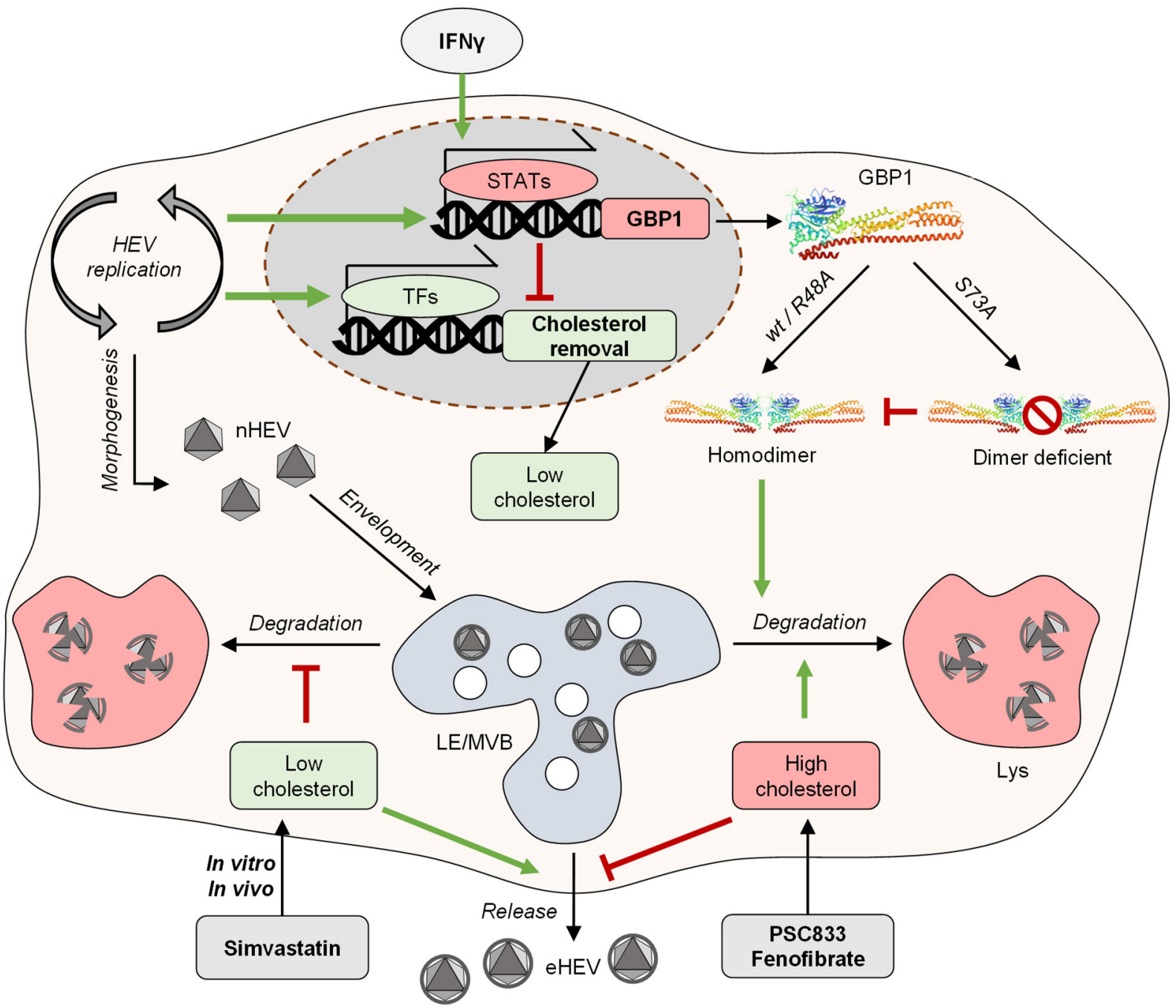

Figure 50. Schematic representation of antiviral activities identified in this study. An HEV infection, similar as an IFN $\gamma$-stimulation, induces the ISG GBP1. Acting through homodimerisation, this host-factor induces lysosomal degradation of the virus. Disruption of homodimerisation-capacity leads to loss of GBP1-mediated lysosomal HEVdegradation. On the other hand, an HEV-infection results, opposing to IFN $\gamma$-stimulation, in lowered cellular cholesterol levels via dysregulation of related gene-expression. This favours HEV-egress from MVBs and can be induced by inhibiting cholesterol-biosynthesis via Simvastatin both in vitro and in chronically HEV-infected patients. On the contrary, elevating intracellular cholesterol levels e.g. via drugs such as PSC833 or Fenofibrate, again leads to lysosomal degradation of $\mathrm{HEV}$ and a subsequent drop in virion-release. nHEV, naked capsid HEV; eHEV, quasi-enveloped HEV; Lys, lysosome; LE, late endosome; MVB, multivesicular body; wt, GBP1 wild-type; R48A, GTPase-deficient GBP1 mutant R48A; S73A, dimerisation-deficient GBP1 mutant S73A; TFs, transcription factors; STATs, signal transducers and activators of transcription. Antiviral activities are coloured red, proviral activities are coloured green. 


\section{Zusammenfassung}

Das Hepatitis-E-Virus ist ein hepatotropes, (+)-Strang ssRNA Virus und das Einzige Mitglied der Familie der Hepeviridae. Fünf humanpathogene Genotypen der Spezies Orthohepevirus A sind beschrieben, wobei Genotyp 1, 2 und 7 zoonotisches Potential aufweisen. Dies hat Implikationen für Übertragungswege und die weltweite Verbreitung des Virus. Während Genotypen 1 und 2 vermehrt in Entwicklungsländern auftreten, sind zoonotische Genotypen vermehrt in Industriestaaten verbreitet. Basis hierfür ist eine klassische Schmierinfektion bzw. fäkal-orale Übertragung als Weg der Transmission. So dienen kontaminierte Wasserquellen, wie auch kontaminiertes Fleisch infizierter Tiere als Reservoir humaner Infektionen. Dieser Sachverhalt führte in der Vergangenheit zu einer Fehleinschätzung der Infektionsrisiken, da primär Länder mit unzureichender Hygiene als endemische Gebiete der viralen Infektionen beschrieben wurden. Jedoch stellt die Übertragung über Nahrungsmittel, nebst Transmissionen über kontaminierte Blutprodukte, ein massives Risiko für entwickelte Länder dar. Mit jährlich über 20 Millionen Neuinfektionen und 44,000 assoziierten Todesfällen, gilt HEV als Hauptauslöser einer akuten, viralen Hepatitis. Während Mortalitätsraten in gesunden Erwachsenen zwischen 1-4 \% schwanken, so erreichen diese in Schwangeren Werte von bis zu 30 \%. Dies ist darin begründet, dass in dieser Gruppe ein fulminanter Verlauf der Hepatitis deutlich häufiger induziert wird. Neben der akuten Hepatitis spielt auch ein chronischer Krankheitsverlauf eine wichtige Rolle in der Pathogenese. Betroffen hiervon sind insbesondere immunsupprimierte Patienten, beispielsweise solche, die eine Organtransplantation erhalten haben. Da generell nur sehr wenig über den viralen Lebenszyklus bekannt ist, sind Behandlungsoptionen entsprechend dünn gesät. Lediglich zwei Medikamente werden hierzu herangezogen: das Virostatikum Ribavirin, ein Nucleosid-Analagon, oder pegyliertes Interferon. Beide können schwerwiegende Nebenwirkungen induzieren, während die Nutzung von Ribavirin zusätzlich eine Medikamentenresistenz in der viralen RNA-Polymerase des HEV induzieren kann. Eine detailliertere Untersuchung des HEV Lebenszyklus ist deshalb zwingend notwendig, da neue Therapieoptionen dringend benötigt werden.

Während einer jeden Virusinfektion, erkennen Zellen virale Komponenten wie Lipide, Proteine oder Nukleinsäuren via PRRs. Im Falle von HEV sind diese hauptsächlich repräsentiert durch TLRs und RLRs wie TLR3/7 und RIG-I. Eine von vielen Folgen der resultierenden Signalkaskade ist die Produktion von IFN $\gamma$. Dieses wiederum induziert im Zuge der angeborenen Immunantwort ISGs, welche großteilig Effektoren des Immunsystems auf zellulärer Ebene sind. Diese ISGs regulieren eine Vielzahl intrazellulärer Signalwege und Prozesse, welche letzten Endes beispielsweise zum Abbau infizierender Pathogene führen. Eines dieser ISGs wird dargestellt durch die 
GTPase GBP1. Als Mitglied der Dynamin Superfamilie besitzt es eine GTPase-Domäne und eine $\alpha$-helikale Effektordomäne. Nach GTP-Bindung homodimerisiert GBP1, was eine C-terminale Farnesylierung nach sich zieht. Diese bedingt, dass GBP1 nun als peripheres Membranprotein vorliegt, wo es oligomerisiert und unter GTP-Hydrolyse große Konformationsänderungen durchläuft. Als Konsequenz werden so Effekte auf die assoziierte Membran ausgeübt, welche beispielsweise Fusion oder Fission bedingen können. Mehrere essenzielle Aminosäuren wurden in diesem Kontext identifiziert. Eine Mutation an Arginin 48 (R48A) führt dazu, dass GBP1 seine Kapazität, GTP zu hydrolysieren, verliert, wobei die Bindung des Nucleotids nicht affektiert wird. Andererseits führt eine Mutation in Serin 73 (S73A) dazu, dass GBP1 keine Homodimere mehr bilden kann. Beide Mutationen bedingen Änderungen im Spektrum der Aufgaben, die von GBP1 erfüllt werden. Hierbei spielen vor allem zwei Aufgaben von GBP1 eine wichtige Rolle: (i) die durch GTP-Hydrolyse bedingte Aktivität als Dynamin, was Auswirkungen auf Membranstrukturen hat und (ii) die Dimerisierungs- und Farnesylierungs-bedingte Rolle als peripheres Membranprotein und damit eines Adapterproteins für degradative Prozesse. Eine Analyse des Wechselspiels von HEV mit IFN $\gamma$ und GBP1 war Zielsetzung des ersten Teils dieser Arbeit, wobei ein besonderes Augenmerk auf den Wirkmechanismus des ISG gelegt wurde.

Durch eine persistente HEV-Infektion in A549 Zellen, sind besonders ISREPromotorelemente induziert. Dies führt dazu, dass auch der ISRE- und GASenthaltende, proximale GBP1-Promotor durch eine HEV-Infektion induziert ist. Interessanterweise spiegelt die Menge an GBP1-spezifischer mRNA diese Induktion nicht wider. Hier führt sowohl eine frühe als auch eine persistente Infektion zu einer Reduktion in der Menge an GBP1 Transkripten. Noch komplexer wird dieser Sachverhalt in Bezug auf die GBP1 Proteinmenge. Diese ist nach Infektion deutlich erhöht, was nahelegt, dass HEV mit der Induzierbarkeit von ISGs interferiert. Eine Begründung für erhöhte GBP1 Proteinmengen kann im persistent HEV-infizierten System darin gefunden werden, dass die Halbwertszeit des Proteins stabilisiert ist. Als Konsequenz einer HEV-Infektion wird also GBP1 als ISG induziert. Ob dem eine antivirale Rolle zugesprochen werden kann, wurde durch eine Überexpression von GBP1 untersucht. Hierbei führt abundantes GBP1 dazu, dass die Menge an intrazellulärem HEV Kapsidprotein, pORF2, sinkt. Als Konsequenz hieraus, sinkt auch die Menge an freigesetzten Virionen drastisch. Welcher Mechanismus dieser Reduktion zugrunde liegt, wurde in CLSM Analysen untersucht. Hierfür wurde in GBP1-überexpreimierenden Zellen sowohl pORF2 als auch das lysosomale Protein LAMP2 observiert, um Lysosomen sichtbar zu machen. Hierbei akkumuliert HEV in punktartigen Strukturen, welche ebenfalls durch Anwesenheit von LAMP2 charakterisiert sind. Dies führt zu einer erhöhten Kolokalisation des viralen Proteins 
mit Lysosomen. Zusätzlich hebt eine Inhibition lysosomaler Degradation via Nutzung von Leupeptin die GBP1-induzierte Reduktion von intrazellulärem pORF2 auf. Dies legt nahe, dass HEV GBP1-abhängig in Lysosomen abgebaut wird. Dieser antivirale Effekt von GBP1 wird auch nach Einführung der GBP1-Mutation R48A beibehalten. Eine Abhängigkeit von der GTPase-Aktivität kann somit ausgeschlossen werden. Andererseits führt eine GBP1-Mutation in Form von S73A zu einem Verlust der antiviralen Aktivitäten, was bedeutet, dass GBP1-Homodimere für eine effiziente Inkorporation von HEV in Lysosomen benötigt wird.

Da GBP1 nur ein Vertreter von vielen verschiedenen ISGs repräsentiert, wurde dessen Rolle innerhalb einer IFN $\gamma$-Stimulation untersucht. Letzteres weist den stärksten antiviralen Effekt im Vergleich zu IFN $\alpha$ und IFN $\beta$ auf, ebenso wie die stärkste Induktion von GBP1. Das Typ II Interferon allein führt zu einer deutlichen Reduktion an intrazellulärem pORF2 und zu reduzierten, viralen Titern im Zellkulturüberstand. Ebenso wie eine GBP1-Überexpression ist dies zurückzuführen auf lysosomale Lokalisation des Kapsidproteins gepaart mit anschließender Degradation. Eine siRNA-basierte Interferenz mit GBP1 führt hier dazu, dass IFN $\gamma$ seine Aktivität gegen HEV nahezu vollständig verliert. Dies ist darin begründet, dass auch eine verminderte Lokalisation in Lysosomen mit dieser Interferenz einhergeht. Zusammenfassend lässt sich schlussfolgern, dass GBP1 einen essenziellen Faktor in der IFN $\gamma$-vermittelten, angeborenen Immunantwort darstellt. Es verübt seine Aufgabe über eine Homodimerabhängige Inkorporation von HEV Virionen in Lysosomen, wo diese abgebaut werden.

Nebst den Effekten, die durch GBP1 bedingt werden, führt eine IFN $\gamma$-Stimulation HEV-infizierter Zellen zu einer Akkumulation von Cholesterin in Lysosomen, was einen weiteren Wirkmechanismus des Cytokins impliziert. Cholesterin spielt eine zentrale Rolle für die Morphogenese, Reifung und den Transport endosomaler Strukturen. Diese wiederum sind von äußerster Wichtigkeit für HEV, da das Virus diese für seine Freisetzung via Exosomen nutzt, indem es über das virale pORF3 mit TSG101 interagiert und Kapside ESCRT-abhängig in MVBs umhüllt werden. Als zweite Zielsetzung dieser Arbeit wurde deshalb angestrebt, die Wechselwirkungen zwischen HEV und intrazellulärem Cholesterins genauer zu charakterisieren.

Eine HEV-Infektion bedingt in A549 Zellen eine Umstrukturierung der Genexpression Cholesterin-assoziierter Faktoren. Auffällig hierbei ist eine deutliche Induktion von Wirtsfaktoren, welche einen Export des Lipids nach sich ziehen. Umgekehrt werden Gene repressiert, welche zu einer Akkumulation intrazellulären Cholesterins führen. Dies hat zur Folge, dass, basierend auf CLSM-Analysen, die intrazelluläre Menge an Cholesterin in infizierten Zellen deutlich abnimmt. Nicht nur in Zellkultur kann ein solcher Effekt beobachtet werden. Auch Serum-Lipide von Patienten sinken mit einer 
HEV Infektion. Hierbei sind sowohl Triglyceride als auch LDL-Cholesterin und totales Cholesterin negativ beeinflusst. Gemeinsam mit in vitro Daten impliziert dies, dass geringe intrazelluläre Cholesterinkonzentrationen einen Vorteil für das Virus darstellen könnten. Um diesen Sachverhalt weiter zu beleuchten, wurden infizierte Zellen einer Modulation zellulären Cholesterins ausgesetzt. Während 25-HC und LDL dazu dienten, höhere Konzentrationen des Lipids zu induzieren, wurde Simvastatin genutzt, um dessen Biosynthese zu hemmen. Eine Bestätigung der zugrundeliegenden Hypothese wurde darin gefunden, dass Simvastatin die Freisetzung von HEV Virionen, unter Abnahme intrazellulären Kapsidproteins, deutlich erhöhte. Gleiches wurde erneut für chronisch-infizierte Transplantationspatienten nachgewiesen, in denen eine Behandlung mit Statinen zu erhöhten, viralen Titern im Serum führt. Gerade für die klinische Evaluation möglicher Therapieoptionen ist dieser Sachverhalt von äußerster Wichtigkeit, da Statine demnach kontraindiziert sein könnten. Im Kontrast zu reduzierten Cholesterinspiegeln, welche von Vorteil für HEV sind, sind erhöhte Cholesterinspiegel nachweislich schädlich für dessen Lebenszyklus. Sowohl unter Anwendung von 25-HC als auch unter LDL-Supplementierung, ist die Menge an intrazellulärem HEV pORF2 reduziert. Erneut hat dies zur Folge, dass die Anzahl freigesetzter Virionen dramatisch sinkt. Ähnlich der phänotypischen Untersuchung einer IFN $\gamma$-Behandlung, zog eine Erhöhung intrazellulären Cholesterins eine Akkumulation von pORF2 in punktartigen Strukturen nach sich. Diese konnten mittels Detektion von LAMP2 als Lysosomen bestätigt werden. In diesem Kontext wurde die lysosomale Aktivität mittels Supplementierung von Leupeptin als ProteaseInhibitor gehemmt. Die Konsequenz hieraus ist eine Aufhebung der reduzierenden Effekte, die durch 25-HC und LDL ausgeübt werden. Zusammengefasst legen diese Daten nahe, dass HEV für eine effiziente Freisetzung von Virionen niedrige intrazelluläre Cholesterinspiegel benötigt. Im Gegensatz hierzu bedingen hohe intrazelluläre Cholesterinkonzentrationen einen lysosomalen Abbau des Virus. Dies öffnet die Tür für eine neue, antivirale Strategie: die pharmakologische Induktion viralen Abbaus unter Benutzung von Cholesterin-Modulatoren.

Um das oben genannte Ziel zu erreichen, wurden verschiedene Substanzen verwendet, die entweder schon als Medikament zugelassen oder Objekt klinischer Studien sind. Diese umfassten die PPAR $\alpha$-Agonisten Fenofibrat und Gemfibrozil, den ABCA1-Inhibitor PSC833, den ACAT-Inhibitor Avasimib, den CYP7A1-Suppressor FGF19 und den PSCK9-Inhibitor Alirocumab. All diese Substanzen haben als mechanistische Grundlage, dass sie als systemische Cholesterinsenker wirken, indem sie eine Retention bzw. eine vermehrte Aufnahme von Cholesterin in die Zelle bedingen. Aus diesem Grund wurden Effekte der Medikamente in Bezug auf virale Freisetzung untersucht. Weder Gemfibrozil noch FGF19 oder Avasimib führten in diesem Kontext zu nennenswerten antiviralen Effekten. Die Verwendung von 
Alirocumab resultierte in einer Reduktion in extrazellulärer RNA und entsprechend viraler Partikel. Besonders jedoch im Falle einer PSC833- und Fenofibrat-Behandlung konnte eine drastische Reduktion in viralen Titern detektiert werden. Auch die Evaluation intrazellulärer Cholesterinkonzentrationen wies im Falle dieser beiden Substanzen einen deutlichen Anstieg und eine Akkumulation des Lipids nach, jedoch nicht für, auf HEV bezogene, inaktive Substanzen. Weiterhin konnten PSC833 und Fenofibrat als Substanzen identifiziert werden, die effizient die Menge an viralem pORF2 reduzieren. Dies korreliert, äquivalent zu der primären Hypothese, mit lysosomaler Lokalisation des Kapsidproteins, wie durch CLSM Analysen belegt wurde. Entsprechend konnte die Applikation von Leupeptin diese reduktiven Effekte effizient unterbinden. Es kann deshalb geschlussfolgert werden, dass sowohl PSC833 als auch Fenofibrat dramatische antivirale Effekte auf HEV ausüben. Der grundlegende Mechanismus hierbei ist die Akkumulation von Cholesterin in infizierten Zellen, was letzten Endes zum lysosomalen Abbau der Viren führt.

Zusammenfassend lässt sich über die vorgelegte Arbeit sagen, dass zwei Grundbestandteile des HEV-Lebenszyklus näher beleuchtet wurden. Die IFN $\gamma$ induzierte GTPase GBP1 wurde als essenzieller Faktor innerhalb der Typ II Interferonvermittelten, angeborenen Immunantwort identifiziert. Es übt diese Rolle aus, indem es via Homodimerisierung die Inkorporation viraler Partikel in Lysosomen induziert, wo diese abgebaut werden. Die Applikation von IFN $\gamma$ zog außerdem eine lysosomale Akkumulation von Cholesterin nach sich, was einen weiteren potenziellen antiviralen Effekt darstellen könnte. In diesem Kontext wurde nachgewiesen, dass HEV niedrige intrazelluläre Cholesterinspiegel für eine effiziente Freisetzung benötigt und dies effizient via Genexpression von Wirtsfaktoren steuern kann. Eine Gabe von Simvastatin im Kontext einer chronischen Infektion in Patienten könnte deshalb kontraindiziert sein, was bedeutend für klinische Behandlungen ist. Eine Erhöhung intrazellulären Cholesterins führt im Gegensatz, ähnlich zu einer Stimulation mit IFN $\gamma$, zum lysosomalen Abbau viraler Partikel. Dieser Sachverhalt wurde erfolgreich genutzt, um einen potenziellen, neuen, antiviralen Wirkstoff zu entdecken: Fenofibrat. Die hier vorgestellte Studie trägt maßgeblich dem Verständnis endolysosomaler Prozesse im Hinblick auf eine HEV-Infektion bei und hat das Potential, die Einführung eines neuen Medikamentes gegen die virale Infektion zu fördern. 


\section{References}

1. Khuroo, M.S. Study of an epidemic of non-A, non-B hepatitis. Possibility of another human hepatitis virus distinct from post-transfusion non-A, non-B type. Am. J. Med. 1980, 68, 818-824, doi:10.1016/0002-9343(80)90200-4.

2. Balayan, M.S.; Andjaparidze, A.G.; Savinskaya, S.S.; Ketiladze, E.S.; Braginsky, D.M.; Savinov, A.P.; Poleschuk, V.F. Evidence for a virus in non-A, non-B hepatitis transmitted via the fecal-oral route. Intervirology 1983, 20, 23-31, doi:10.1159/000149370.

3. Sreenivasan, M.A.; Arankalle, V.A.; Sehgal, A.; Pavri, K.M. Non-A, non-B epidemic hepatitis: visualization of virus-like particles in the stool by immune electron microscopy. J. Gen. Virol. 1984, 65 (Pt 5), 1005-1007, doi:10.1099/00221317-65-5-1005.

4. Smith, D.B.; Simmonds, P.; Members, O.T.I.C.O.T.T.O.V.S.G.; Jameel, S.; Emerson, S.U.; Harrison, T.J.; Meng, X.-J.; Okamoto, H.; van der Poel, W.H.M.; Purdy, M.A. Consensus proposals for classification of the family Hepeviridae. J. Gen. Virol. 2014, 95, 2223-2232, doi:10.1099/vir.0.068429-0.

5. Thiry, D.; Mauroy, A.; Pavio, N.; Purdy, M.A.; Rose, N.; Thiry, E.; Oliveira-Filho, E.F. de. Hepatitis E Virus and Related Viruses in Animals. Transbound. Emerg. Dis. 2017, 64, 37-52, doi:10.1111/tbed.12351.

6. Doceul, V.; Bagdassarian, E.; Demange, A.; Pavio, N. Zoonotic Hepatitis E Virus: Classification, Animal Reservoirs and Transmission Routes. Viruses 2016, 8, doi:10.3390/v8100270.

7. Primadharsini, P.P.; Nagashima, S.; Okamoto, H. Genetic Variability and Evolution of Hepatitis E Virus. Viruses 2019, 11, doi:10.3390/v11050456.

8. Sridhar, S.; Teng, J.L.L.; Chiu, T.-H.; Lau, S.K.P.; Woo, P.C.Y. Hepatitis E Virus Genotypes and Evolution: Emergence of Camel Hepatitis E Variants. Int. J. Mol. Sci. 2017, 18, doi:10.3390/ijms18040869.

9. Tam, A.W.; Smith, M.M.; Guerra, M.E.; Huang, C.C.; Bradley, D.W.; Fry, K.E.; Reyes, G.R. Hepatitis E virus (HEV): molecular cloning and sequencing of the full-length viral genome. Virology 1991, 185, 120-131, doi:10.1016/00426822(91)90760-9.

10. Decroly, E.; Ferron, F.; Lescar, J.; Canard, B. Conventional and unconventional mechanisms for capping viral mRNA. Nat. Rev. Microbiol. 2011, 10, 51-65, doi:10.1038/nrmicro2675.

11. Nanda, S.K.; Panda, S.K.; Durgapal, H.; Jameel, S. Detection of the negative strand of hepatitis E virus RNA in the livers of experimentally infected rhesus monkeys: evidence for viral replication. J. Med. Virol. 1994, 42, 237-240, doi:10.1002/jmv.1890420306. 
12. Ahmad, I.; Holla, R.P.; Jameel, S. Molecular virology of hepatitis E virus. Virus Res. 2011, 161, 47-58, doi:10.1016/j.virusres.2011.02.011.

13. Graff, J.; Torian, U.; Nguyen, H.; Emerson, S.U. A bicistronic subgenomic mRNA encodes both the ORF2 and ORF3 proteins of hepatitis E virus. J. Virol. 2006, 80, 5919-5926, doi:10.1128/JVI.00046-06.

14. Ju, X.; Xiang, G.; Gong, M.; Yang, R.; Qin, J.; Li, Y.; Nan, Y.; Yang, Y.; Zhang, Q.C.; Ding, Q. Identification of functional cis-acting RNA elements in the hepatitis E virus genome required for viral replication. PLoS Pathog. 2020, 16, e1008488, doi:10.1371/journal.ppat.1008488.

15. Nair, V.P.; Anang, S.; Subramani, C.; Madhvi, A.; Bakshi, K.; Srivastava, A.; Shalimar; Nayak, B.; Ranjith Kumar, C.T.; Surjit, M. Endoplasmic Reticulum Stress Induced Synthesis of a Novel Viral Factor Mediates Efficient Replication of Genotype-1 Hepatitis E Virus. PLoS Pathog. 2016, 12, e1005521, doi:10.1371/journal.ppat.1005521.

16. Koonin, E.V.; Gorbalenya, A.E.; Purdy, M.A.; Rozanov, M.N.; Reyes, G.R.; Bradley, D.W. Computer-assisted assignment of functional domains in the nonstructural polyprotein of hepatitis E virus: delineation of an additional group of positive-strand RNA plant and animal viruses. Proc. Natl. Acad. Sci. U. S. A. 1992, 89, 8259-8263, doi:10.1073/pnas.89.17.8259.

17. Magden, J.; Takeda, N.; Li, T.; Auvinen, P.; Ahola, T.; Miyamura, T.; Merits, A.; Kääriäinen, L. Virus-specific mRNA capping enzyme encoded by hepatitis E virus. J. Virol. 2001, 75, 6249-6255, doi:10.1128/JVI.75.14.6249-6255.2001.

18. Agrawal, S.; Gupta, D.; Panda, S.K. The 3' end of hepatitis E virus (HEV) genome binds specifically to the viral RNA-dependent RNA polymerase (RdRp). Virology 2001, 282, 87-101, doi:10.1006/viro.2000.0819.

19. Sehgal, D.; Thomas, S.; Chakraborty, M.; Jameel, S. Expression and processing of the Hepatitis E virus ORF1 nonstructural polyprotein. Virol. J. 2006, 3, 38, doi:10.1186/1743-422X-3-38.

20. Parvez, M.K. The hepatitis E virus nonstructural polyprotein. Future Microbiol. 2017, 12, 915-924, doi:10.2217/fmb-2017-0016.

21. Parvez, M.K. The hepatitis E virus ORF1 'X-domain' residues form a putative macrodomain protein/Appr-1"-pase catalytic-site, critical for viral RNA replication. Gene 2015, 566, 47-53, doi:10.1016/j.gene.2015.04.026.

22. Parvez, M.K. Mutational analysis of hepatitis E virus ORF1 "Y-domain": Effects on RNA replication and virion infectivity. World J. Gastroenterol. 2017, 23, 590602, doi:10.3748/wjg.v23.i4.590.

23. Johne, R.; Reetz, J.; Ulrich, R.G.; Machnowska, P.; Sachsenröder, J.; Nickel, P.; Hofmann, J. An ORF1-rearranged hepatitis E virus derived from a chronically infected patient efficiently replicates in cell culture. J. Viral Hepat. 2014, 21, 447456, doi:10.1111/jvh.12157. 
24. Pudupakam, R.S.; Kenney, S.P.; Córdoba, L.; Huang, Y.-W.; Dryman, B.A.; Leroith, T.; Pierson, F.W.; Meng, X.-J. Mutational analysis of the hypervariable region of hepatitis e virus reveals its involvement in the efficiency of viral RNA replication. J. Virol. 2011, 85, 10031-10040, doi:10.1128/JVI.00763-11.

25. Surjit, M.; Jameel, S.; Lal, S.K. The ORF2 protein of hepatitis E virus binds the $5^{\prime}$ region of viral RNA. J. Virol. 2004, 78, 320-328, doi:10.1128/jvi.78.1.320-328.2004.

26. Montpellier, C.; Wychowski, C.; Sayed, I.M.; Meunier, J.-C.; Saliou, J.-M.; Ankavay, M.; Bull, A.; Pillez, A.; Abravanel, F.; Helle, F.; et al. Hepatitis E Virus Lifecycle and Identification of 3 Forms of the ORF2 Capsid Protein. Gastroenterology 2018, 154, 211-223.e8, doi:10.1053/j.gastro.2017.09.020.

27. Zafrullah, M.; Ozdener, M.H.; Kumar, R.; Panda, S.K.; Jameel, S. Mutational analysis of glycosylation, membrane translocation, and cell surface expression of the hepatitis E virus ORF2 protein. J. Virol. 1999, 73, 4074-4082, doi:10.1128/JVI.73.5.4074-4082.1999.

28. Xu, M.; Behloul, N.; Wen, J.; Zhang, J.; Meng, J. Role of asparagine at position 562 in dimerization and immunogenicity of the hepatitis $\mathrm{E}$ virus capsid protein. Infect. Genet. Evol. 2016, 37, 99-107, doi:10.1016/j.meegid.2015.11.006.

29. Graff, J.; Zhou, Y.-H.; Torian, U.; Nguyen, H.; St Claire, M.; Yu, C.; Purcell, R.H.; Emerson, S.U. Mutations within potential glycosylation sites in the capsid protein of hepatitis E virus prevent the formation of infectious virus particles. $J$. Virol. 2008, 82, 1185-1194, doi:10.1128/JVI.01219-07.

30. Ankavay, M.; Montpellier, C.; Sayed, I.M.; Saliou, J.-M.; Wychowski, C.; Saas, L.; Duvet, S.; Aliouat-Denis, C.-M.; Farhat, R.; Masson d'Autume, V. de; et al. New insights into the ORF2 capsid protein, a key player of the hepatitis $E$ virus lifecycle. Sci. Rep. 2019, 9, 6243, doi:10.1038/s41598-019-42737-2.

31. Li, T.-C.; Takeda, N.; Miyamura, T.; Matsuura, Y.; Wang, J.C.Y.; Engvall, H.; Hammar, L.; Xing, L.; Cheng, R.H. Essential elements of the capsid protein for self-assembly into empty virus-like particles of hepatitis E virus. J. Virol. 2005, 79, 12999-13006, doi:10.1128/JVI.79.20.12999-13006.2005.

32. Emerson, S.U.; Nguyen, H.; Torian, U.; Purcell, R.H. ORF3 protein of hepatitis E virus is not required for replication, virion assembly, or infection of hepatoma cells in vitro. J. Virol. 2006, 80, 10457-10464, doi:10.1128/JVI.00892-06.

33. Ding, Q.; Heller, B.; Capuccino, J.M.V.; Song, B.; Nimgaonkar, I.; Hrebikova, G.; Contreras, J.E.; Ploss, A. Hepatitis E virus ORF3 is a functional ion channel required for release of infectious particles. Proc. Natl. Acad. Sci. U. S. A. 2017, 114, 1147-1152, doi:10.1073/pnas.1614955114.

34. Nagashima, S.; Takahashi, M.; Jirintai; Tanaka, T.; Yamada, K.; Nishizawa, T.; Okamoto, H. A PSAP motif in the ORF3 protein of hepatitis E virus is necessary for virion release from infected cells. J. Gen. Virol. 2011, 92, 269-278, doi:10.1099/vir.0.025791-0. 
35. Osterman, A.; Vizoso Pinto, M.G.; Haase, R.; Nitschko, H.; Jäger, S.; Sander, M.; Motz, M.; Mohn, U.; Baiker, A. Systematic screening for novel, serologically reactive Hepatitis E Virus epitopes. Virol. J. 2012, 9, 28, doi:10.1186/1743-422X-928.

36. Gouttenoire, J.; Pollán, A.; Abrami, L.; Oechslin, N.; Mauron, J.; Matter, M.; Oppliger, J.; Szkolnicka, D.; Dao Thi, V.L.; van der Goot, F.G.; et al. Palmitoylation mediates membrane association of hepatitis E virus ORF3 protein and is required for infectious particle secretion. PLoS Pathog. 2018, 14, e1007471, doi:10.1371/journal.ppat.1007471.

37. Zafrullah, M.; Ozdener, M.H.; Panda, S.K.; Jameel, S. The ORF3 protein of hepatitis E virus is a phosphoprotein that associates with the cytoskeleton. J. Virol. 1997, 71, 9045-9053, doi:10.1128/JVI.71.12.9045-9053.1997.

38. Tyagi, S.; Korkaya, H.; Zafrullah, M.; Jameel, S.; Lal, S.K. The phosphorylated form of the ORF3 protein of hepatitis E virus interacts with its non-glycosylated form of the major capsid protein, ORF2. J. Biol. Chem. 2002, 277, 22759-22767, doi:10.1074/jbc.M200185200.

39. VISHWANATHAN, R. Infectious hepatitis in Delhi (1955-56) : a critical study : epidemiology. Ind J Med Res 1957, 45, 49-58.

40. Maila, H.T.; Bowyer, S.M.; Swanepoel, R. Identification of a new strain of hepatitis E virus from an outbreak in Namibia in 1995. J. Gen. Virol. 2004, 85, 8995, doi:10.1099/vir.0.19587-0.

41. Pavio, N.; Merbah, T.; Thébault, A. Frequent hepatitis E virus contamination in food containing raw pork liver, France. Emerg. Infect. Dis. 2014, 20, 1925-1927, doi:10.3201/eid2011.140891.

42. Harrison, L.'C.; DiCaprio, E. Hepatitis E Virus: An Emerging Foodborne Pathogen. Front. Sustain. Food Syst. 2018, 2, 263, doi:10.3389/fsufs.2018.00014.

43. Colson, P.; Coze, C.; Gallian, P.; Henry, M.; Micco, P. de; Tamalet, C. Transfusion-associated hepatitis E, France. Emerg. Infect. Dis. 2007, 13, 648-649, doi:10.3201/eid1304.061387.

44. Bi, H.; Yang, R.; Wu, C.; Xia, J. Hepatitis E virus and blood transfusion safety. Epidemiol. Infect. 2020, 148, e158, doi:10.1017/S0950268820001429.

45. Khuroo, M.S.; Kamili, S.; Jameel, S. Vertical transmission of hepatitis E virus. Lancet 1995, 345, 1025-1026, doi:10.1016/s0140-6736(95)90761-0.

46. Khuroo, M.S.; Kamili, S. Clinical course and duration of viremia in vertically transmitted hepatitis E virus (HEV) infection in babies born to HEV-infected mothers. J. Viral Hepat. 2009, 16, 519-523, doi:10.1111/j.1365-2893.2009.01101.x.

47. Sharma, S.; Kumar, A.; Kar, P.; Agarwal, S.; Ramji, S.; Husain, S.A.; Prasad, S. Risk factors for vertical transmission of hepatitis E virus infection. J. Viral Hepat. 2017, 24, 1067-1075, doi:10.1111/jvh.12730. 
48. Carratalà, A.; Joost, S. Population density and water balance influence the global occurrence of hepatitis E epidemics. Sci. Rep. 2019, 9, 10042, doi:10.1038/s41598019-46475-3.

49. Howard, C.M.; Handzel, T.; Hill, V.R.; Grytdal, S.P.; Blanton, C.; Kamili, S.; Drobeniuc, J.; Hu, D.; Teshale, E. Novel risk factors associated with hepatitis E virus infection in a large outbreak in northern Uganda: results from a casecontrol study and environmental analysis. Am. J. Trop. Med. Hyg. 2010, 83, 11701173, doi:10.4269/ajtmh.2010.10-0384.

50. Pérez-Gracia, M.T.; Suay, B.; Mateos-Lindemann, M.L. Hepatitis E: an emerging disease. Infect. Genet. Evol. 2014, 22, 40-59, doi:10.1016/j.meegid.2014.01.002.

51. Khuroo, M.S.; Khuroo, M.S.; Khuroo, N.S. Transmission of Hepatitis E Virus in Developing Countries. Viruses 2016, 8, doi:10.3390/v8090253.

52. Chandra, V.; Taneja, S.; Kalia, M.; Jameel, S. Molecular biology and pathogenesis of hepatitis E virus. J. Biosci. 2008, 33, 451-464, doi:10.1007/s12038-008-0064-1.

53. Aggarwal, R.; Naik, S. Epidemiology of hepatitis E: current status. J. Gastroenterol. Hepatol. 2009, 24, 1484-1493, doi:10.1111/j.1440-1746.2009.05933.x.

54. Jefferies, M.; Rauff, B.; Rashid, H.; Lam, T.; Rafiq, S. Update on global epidemiology of viral hepatitis and preventive strategies. World J. Clin. Cases 2018, 6, 589-599, doi:10.12998/wjcc.v6.i13.589.

55. Wilhelm, B.; Waddell, L.; Greig, J.; Young, I. Systematic review and metaanalysis of the seroprevalence of hepatitis $\mathrm{E}$ virus in the general population across non-endemic countries. PLoS One 2019, 14, e0216826, doi:10.1371/journal.pone.0216826.

56. Bouamra, Y.; Gérolami, R.; Arzouni, J.-P.; Grimaud, J.-C.; Lafforgue, P.; Nelli, M.; Tivoli, N.; Ferretti, A.; Motte, A.; Colson, P. Emergence of autochthonous infections with hepatitis E virus of genotype 4 in Europe. Intervirology 2014, 57, 43-48, doi:10.1159/000354801.

57. Holla, R.P.; Ahmad, I.; Ahmad, Z.; Jameel, S. Molecular virology of hepatitis E virus. Semin. Liver Dis. 2013, 33, 3-14, doi:10.1055/s-0033-1338110.

58. Wehmeyer, M.H.; Hartl, J.; Wulffen, M. von; Lohse, A.W.; Pischke, S. Zeitlicher Verlauf von gemeldeten Fällen und Publikationen: Hepatitis E im Vergleich zu Hepatitis A - D In Deutschland (2001 bis 2016). Z. Gastroenterol. 2018, 56, 29-35, doi:10.1055/s-0043-123830.

59. Aggarwal, R. Hepatitis E: Historical, contemporary and future perspectives. J. Gastroenterol. Hepatol. 2011, 26 Suppl 1, 72-82, doi:10.1111/j.14401746.2010.06540.x.

60. Purcell, R.H.; Emerson, S.U. Hepatitis E: an emerging awareness of an old disease. J. Hepatol. 2008, 48, 494-503, doi:10.1016/j.jhep.2007.12.008.

61. Riezebos-Brilman, A.; Verschuuren, E.A.M.; van Son, W.J.; van Imhoff, G.W.; Brügemann, J.; Blokzijl, H.; Niesters, H.G.M. The clinical course of hepatitis E 
virus infection in patients of a tertiary Dutch hospital over a 5-year period. J. Clin. Virol. 2013, 58, 509-514, doi:10.1016/j.jcv.2013.08.022.

62. Sarkar, S.; Rivera, E.M.; Engle, R.E.; Nguyen, H.T.; Schechterly, C.A.; Alter, H.J.; Liang, T.J.; Purcell, R.H.; Hoofnagle, J.H.; Ghany, M.G. An Epidemiologic Investigation of a Case of Acute Hepatitis E. J. Clin. Microbiol. 2015, 53, 35473552, doi:10.1128/JCM.01189-15.

63. El Sayed Zaki, M.; El Razek, M.M.A.; El Razek, H.M.A. Maternal-Fetal Hepatitis E Transmission: Is It Underestimated? J. Clin. Transl. Hepatol. 2014, 2, 117-123, doi:10.14218/JCTH.2014.00006.

64. Fousekis, F.S.; Mitselos, I.V.; Christodoulou, D.K. Extrahepatic manifestations of hepatitis E virus: An overview. Clin. Mol. Hepatol. 2020, 26, 16-23, doi:10.3350/cmh.2019.0082.

65. Srivastava, R.; Aggarwal, R.; Sachdeva, S.; Alam, M.I.; Jameel, S.; Naik, S. Adaptive immune responses during acute uncomplicated and fulminant hepatitis E. J. Gastroenterol. Hepatol. 2011, 26, 306-311, doi:10.1111/j.14401746.2010.06356.x.

66. Bose, P.D.; Das, B.C.; Kumar, A.; Gondal, R.; Kumar, D.; Kar, P. High viral load and deregulation of the progesterone receptor signaling pathway: association with hepatitis E-related poor pregnancy outcome. J. Hepatol. 2011, 54, 1107-1113, doi:10.1016/j.jhep.2010.08.037.

67. Yu, W.; Hao, X.; Li, Y.; Yang, C.; Li, Y.; He, Z.; Huang, F. Vertical transmission of hepatitis $\mathrm{E}$ virus in pregnant rhesus macaques. Sci. Rep. 2020, 10, 17517, doi:10.1038/s41598-020-74461-7.

68. Jilani, N.; Das, B.C.; Husain, S.A.; Baweja, U.K.; Chattopadhya, D.; Gupta, R.K.; Sardana, S.; Kar, P. Hepatitis E virus infection and fulminant hepatic failure during pregnancy. J. Gastroenterol. Hepatol. 2007, 22, 676-682, doi:10.1111/j.14401746.2007.04913.x.

69. Julin, C.H.; Hjortaas, K.; Dembinski, J.L.; Sandbu, S.; Øverbø, J.; Stene-Johansen, K.; Dudman, S. Hepatitis E in Pregnant Women and the Potential Use of HEV Vaccine to Prevent Maternal Infection and Mortality. Curr Trop Med Rep 2019, 6, 197-204, doi:10.1007/s40475-019-00193-y.

70. Aggarwal, R.; Aggarwal, R.A. Hepatitis E: clinical presentation in diseaseendemic areas and diagnosis. Semin. Liver Dis. 2013, 33, 30-40, doi:10.1055/s0033-1338112.

71. Gouilly, J.; Chen, Q.; Siewiera, J.; Cartron, G.; Levy, C.; Dubois, M.; Al-Daccak, R.; Izopet, J.; Jabrane-Ferrat, N.; El Costa, H. Genotype specific pathogenicity of hepatitis E virus at the human maternal-fetal interface. Nat. Commun. 2018, 9, 4748, doi:10.1038/s41467-018-07200-2.

72. McPherson, S.; Elsharkawy, A.M.; Ankcorn, M.; Ijaz, S.; Powell, J.; Rowe, I.; Tedder, R.; Andrews, P.A. Summary of the British Transplantation Society UK 
Guidelines for Hepatitis E and Solid Organ Transplantation. Transplantation 2018, 102, 15-20, doi:10.1097/TP.0000000000001908.

73. Kamar, N.; Izopet, J.; Dalton, H.R. Chronic hepatitis e virus infection and treatment. J. Clin. Exp. Hepatol. 2013, 3, 134-140, doi:10.1016/j.jceh.2013.05.003.

74. Kamar, N.; Bendall, R.; Legrand-Abravanel, F.; Xia, N.-S.; Ijaz, S.; Izopet, J.; Dalton, H.R. Hepatitis E. Lancet 2012, 379, 2477-2488, doi:10.1016/S01406736(11)61849-7.

75. Kamar, N.; Selves, J.; Mansuy, J.-M.; Ouezzani, L.; Péron, J.-M.; Guitard, J.; Cointault, O.; Esposito, L.; Abravanel, F.; Danjoux, M.; et al. Hepatitis E virus and chronic hepatitis in organ-transplant recipients. N. Engl. J. Med. 2008, 358, 811-817, doi:10.1056/NEJMoa0706992.

76. Ollier, L.; Tieulie, N.; Sanderson, F.; Heudier, P.; Giordanengo, V.; Fuzibet, J.-G.; Nicand, E. Chronic hepatitis after hepatitis E virus infection in a patient with non-Hodgkin lymphoma taking rituximab. Ann. Intern. Med. 2009, 150, 430-431, doi:10.7326/0003-4819-150-6-200903170-00026.

77. Lhomme, S.; Bardiaux, L.; Abravanel, F.; Gallian, P.; Kamar, N.; Izopet, J. Hepatitis E Virus Infection in Solid Organ Transplant Recipients, France. Emerg. Infect. Dis. 2017, 23, 353-356, doi:10.3201/eid2302.161094.

78. Bouts, A.H.M.; Schriemer, P.J.; Zaaijer, H.L. Chronic hepatitis E resolved by reduced immunosuppression in pediatric kidney transplant patients. Pediatrics 2015, 135, e1075-8, doi:10.1542/peds.2014-3790.

79. Keane, F.; Gompels, M.; Bendall, R.; Drayton, R.; Jennings, L.; Black, J.; Baragwanath, G.; Lin, N.; Henley, W.; Ngui, S.-L.; et al. Hepatitis E virus coinfection in patients with HIV infection. HIV Med. 2012, 13, 83-88, doi:10.1111/j.1468-1293.2011.00942.x.

80. Rivero-Juarez, A.; Lopez-Lopez, P.; Frias, M.; Rivero, A. Hepatitis E Infection in HIV-Infected Patients. Front. Microbiol. 2019, 10, 1425, doi:10.3389/fmicb.2019.01425.

81. Lozano, R.; Naghavi, M.; Foreman, K.; Lim, S.; Shibuya, K.; Aboyans, V.; Abraham, J.; Adair, T.; Aggarwal, R.; Ahn, S.Y.; et al. Global and regional mortality from 235 causes of death for 20 age groups in 1990 and 2010: a systematic analysis for the Global Burden of Disease Study 2010. Lancet 2012, 380, 2095-2128, doi:10.1016/S0140-6736(12)61728-0.

82. Rein, D.B.; Stevens, G.A.; Theaker, J.; Wittenborn, J.S.; Wiersma, S.T. The global burden of hepatitis E virus genotypes 1 and 2 in 2005. Hepatology 2012, 55, 988997, doi:10.1002/hep.25505.

83. Kamar, N.; Abravanel, F.; Garrouste, C.; Cardeau-Desangles, I.; Mansuy, J.M.; Weclawiak, H.; Izopet, J.; Rostaing, L. Three-month pegylated interferon-alpha2a therapy for chronic hepatitis E virus infection in a haemodialysis patient. Nephrol. Dial. Transplant 2010, 25, 2792-2795, doi:10.1093/ndt/gfq282. 
84. Kamar, N.; Rostaing, L.; Abravanel, F.; Garrouste, C.; Esposito, L.; CardeauDesangles, I.; Mansuy, J.M.; Selves, J.; Peron, J.M.; Otal, P.; et al. Pegylated interferon-alpha for treating chronic hepatitis $\mathrm{E}$ virus infection after liver transplantation. Clin. Infect. Dis. 2010, 50, e30-3, doi:10.1086/650488.

85. Yang, J.-F.; Kao, Y.-H.; Dai, C.-Y.; Huang, J.-F.; Hsieh, M.-Y.; Lin, Z.-Y.; Chen, S.C.; Hsieh, M.-Y.; Wang, L.-Y.; Chuang, W.-L.; et al. Comparison of adverse effects related to pegylated interferon-based therapy for patients with chronic hepatitis B and chronic hepatitis C in Taiwan. Hepatol. Int. 2010, 4, 732-740, doi:10.1007/s12072-010-9208-0.

86. Kamar, N.; Rostaing, L.; Abravanel, F.; Garrouste, C.; Lhomme, S.; Esposito, L.; Basse, G.; Cointault, O.; Ribes, D.; Nogier, M.B.; et al. Ribavirin therapy inhibits viral replication on patients with chronic hepatitis e virus infection. Gastroenterology 2010, 139, 1612-1618, doi:10.1053/j.gastro.2010.08.002.

87. Protin, C.; Abravanel, F.; Alric, L.; Tavitian, S.; Obéric, L.; Izopet, J.; MartinBlondel, G.; Ysebaert, L. Ribavirin for Chronic Hepatitis E Virus Infection in Ibrutinib-Exposed Patients. Open Forum Infect. Dis. 2019, 6, ofz345, doi:10.1093/ofid/ofz345.

88. Debing, Y.; Gisa, A.; Dallmeier, K.; Pischke, S.; Bremer, B.; Manns, M.; Wedemeyer, H.; Suneetha, P.V.; Neyts, J. A mutation in the hepatitis E virus RNA polymerase promotes its replication and associates with ribavirin treatment failure in organ transplant recipients. Gastroenterology 2014, 147, 100811.e7; quiz e15-6, doi:10.1053/j.gastro.2014.08.040.

89. Glitscher, M.; Himmelsbach, K.; Woytinek, K.; Johne, R.; Reuter, A.; Spiric, J.; Schwaben, L.; Grünweller, A.; Hildt, E. Inhibition of Hepatitis E Virus Spread by the Natural Compound Silvestrol. Viruses 2018, 10, doi:10.3390/v10060301.

90. Müller, C.; Obermann, W.; Schulte, F.W.; Lange-Grünweller, K.; Oestereich, L.; Elgner, F.; Glitscher, M.; Hildt, E.; Singh, K.; Wendel, H.-G.; et al. Comparison of broad-spectrum antiviral activities of the synthetic rocaglate CR-31-B (-) and the eIF4A-inhibitor Silvestrol. Antiviral Res. 2020, 175, 104706, doi:10.1016/j.antiviral.2020.104706.

91. Kalia, M.; Chandra, V.; Rahman, S.A.; Sehgal, D.; Jameel, S. Heparan sulfate proteoglycans are required for cellular binding of the hepatitis E virus ORF2 capsid protein and for viral infection. J. Virol. 2009, 83, 12714-12724, doi:10.1128/JVI.00717-09.

92. Yu, H.; Li, S.; Yang, C.; Wei, M.; Song, C.; Zheng, Z.; Gu, Y.; Du, H.; Zhang, J.; Xia, N. Homology model and potential virus-capsid binding site of a putative HEV receptor Grp78. J. Mol. Model. 2011, 17, 987-995, doi:10.1007/s00894-0100794-5. 
93. Zheng, Z.-Z.; Miao, J.; Zhao, M.; Tang, M.; Yeo, A.E.T.; Yu, H.; Zhang, J.; Xia, N.S. Role of heat-shock protein 90 in hepatitis E virus capsid trafficking. J. Gen. Virol. 2010, 91, 1728-1736, doi:10.1099/vir.0.019323-0.

94. Zhou, Y.; Emerson, S.U. P.302 Heat shock cognate protein 70 may mediate the entry of hepatitis E virus into host cells. Journal of Clinical Virology 2006, 36, S155, doi:10.1016/S1386-6532(06)80480-4.

95. Yin, X.; Ambardekar, C.; Lu, Y.; Feng, Z. Distinct Entry Mechanisms for Nonenveloped and Quasi-Enveloped Hepatitis E Viruses. J. Virol. 2016, 90, 4232 4242, doi:10.1128/JVI.02804-15.

96. Holla, P.; Ahmad, I.; Ahmed, Z.; Jameel, S. Hepatitis E virus enters liver cells through a dynamin-2, clathrin and membrane cholesterol-dependent pathway. Traffic 2015, 16, 398-416, doi:10.1111/tra.12260.

97. Perttilä, J.; Spuul, P.; Ahola, T. Early secretory pathway localization and lack of processing for hepatitis E virus replication protein pORF1. J. Gen. Virol. 2013, 94, 807-816, doi:10.1099/vir.0.049577-0.

98. Surjit, M.; Jameel, S.; Lal, S.K. Cytoplasmic localization of the ORF2 protein of hepatitis $\mathrm{E}$ virus is dependent on its ability to undergo retrotranslocation from the endoplasmic reticulum. J. Virol. 2007, 81, 3339-3345, doi:10.1128/JVI.02039-06.

99. Li, D.; Huang, R.; Tian, X.; Yin, S.; Wei, J.; Huang, X.; Wang, B.; Li, R.; Li, Y. Morphology and morphogenesis of hepatitis E virus (strain 87A). Chin. Med. J. (Engl) 1995, 108, 126-131.

100. Nagashima, S.; Takahashi, M.; Jirintai, S.; Tanggis; Kobayashi, T.; Nishizawa, T.; Okamoto, $\mathrm{H}$. The membrane on the surface of hepatitis $\mathrm{E}$ virus particles is derived from the intracellular membrane and contains trans-Golgi network protein 2. Arch. Virol. 2014, 159, 979-991, doi:10.1007/s00705-013-1912-3.

101. Kenney, S.P.; Wentworth, J.L.; Heffron, C.L.; Meng, X.-J. Replacement of the hepatitis E virus ORF3 protein PxxP motif with heterologous late domain motifs affects virus release via interaction with TSG101. Virology 2015, 486, 198-208, doi:10.1016/j.virol.2015.09.012.

102. Razi, M.; Futter, C.E. Distinct roles for Tsg101 and Hrs in multivesicular body formation and inward vesiculation. Mol. Biol. Cell 2006, 17, 3469-3483, doi:10.1091/mbc.e05-11-1054.

103. Nagashima, S.; Takahashi, M.; Jirintai, S.; Tanaka, T.; Nishizawa, T.; Yasuda, J.; Okamoto, H. Tumour susceptibility gene 101 and the vacuolar protein sorting pathway are required for the release of hepatitis E virions. J. Gen. Virol. 2011, 92, 2838-2848, doi:10.1099/vir.0.035378-0.

104. Primadharsini, P.P.; Nagashima, S.; Takahashi, M.; Kobayashi, T.; Nishiyama, T.; Nishizawa, T.; Yasuda, J.; Mulyanto; Okamoto, H. Multivesicular body sorting and the exosomal pathway are required for the release of rat hepatitis $E$ virus 
from infected cells. Virus Res. 2020, 278, 197868,

doi:10.1016/j.virusres.2020.197868.

105. Nagashima, S.; Jirintai, S.; Takahashi, M.; Kobayashi, T.; Tanggis; Nishizawa, T.; Kouki, T.; Yashiro, T.; Okamoto, H. Hepatitis E virus egress depends on the exosomal pathway, with secretory exosomes derived from multivesicular bodies. J. Gen. Virol. 2014, 95, 2166-2175, doi:10.1099/vir.0.066910-0.

106. Nagashima, S.; Takahashi, M.; Kobayashi, T.; Nishizawa, T.; Nishiyama, T.; Primadharsini, P.P.; Okamoto, H. Characterization of the Quasi-Enveloped Hepatitis E Virus Particles Released by the Cellular Exosomal Pathway. J. Virol. 2017, 91, doi:10.1128/JVI.00822-17.

107. Yin, X.; Li, X.; Feng, Z. Role of Envelopment in the HEV Life Cycle. Viruses 2016, 8, doi:10.3390/v8080229.

108. Nimgaonkar, I.; Ding, Q.; Schwartz, R.E.; Ploss, A. Hepatitis E virus: advances and challenges. Nat. Rev. Gastroenterol. Hepatol. 2018, 15, 96-110, doi:10.1038/nrgastro.2017.150.

109. Rock, F.L.; Hardiman, G.; Timans, J.C.; Kastelein, R.A.; Bazan, J.F. A family of human receptors structurally related to Drosophila Toll. Proc. Natl. Acad. Sci. U. S. A. 1998, 95, 588-593, doi:10.1073/pnas.95.2.588.

110. Husebye, H.; Halaas, Ø.; Stenmark, H.; Tunheim, G.; Sandanger, Ø.; Bogen, B.; Brech, A.; Latz, E.; Espevik, T. Endocytic pathways regulate Toll-like receptor 4 signaling and link innate and adaptive immunity. EMBO J. 2006, 25, 683-692, doi:10.1038/sj.emboj.7600991.

111. Nilsen, N.J.; Deininger, S.; Nonstad, U.; Skjeldal, F.; Husebye, H.; Rodionov, D.; Aulock, S. von; Hartung, T.; Lien, E.; Bakke, O.; et al. Cellular trafficking of lipoteichoic acid and Toll-like receptor 2 in relation to signaling: role of CD14 and CD36. J. Leukoc. Biol. 2008, 84, 280-291, doi:10.1189/jlb.0907656.

112. Ishii, N.; Funami, K.; Tatematsu, M.; Seya, T.; Matsumoto, M. Endosomal localization of TLR8 confers distinctive proteolytic processing on human myeloid cells. J. Immunol. 2014, 193, 5118-5128, doi:10.4049/jimmunol.1401375.

113. Johnsen, I.B.; Nguyen, T.T.; Ringdal, M.; Tryggestad, A.M.; Bakke, O.; Lien, E.; Espevik, T.; Anthonsen, M.W. Toll-like receptor 3 associates with c-Src tyrosine kinase on endosomes to initiate antiviral signaling. EMBO J. 2006, 25, 3335-3346, doi:10.1038/sj.emboj.7601222.

114. Latz, E.; Schoenemeyer, A.; Visintin, A.; Fitzgerald, K.A.; Monks, B.G.; Knetter, C.F.; Lien, E.; Nilsen, N.J.; Espevik, T.; Golenbock, D.T. TLR9 signals after translocating from the ER to CpG DNA in the lysosome. Nat. Immunol. 2004, 5, 190-198, doi:10.1038/ni1028.

115. Nishiya, T.; Kajita, E.; Miwa, S.; Defranco, A.L. TLR3 and TLR7 are targeted to the same intracellular compartments by distinct regulatory elements. J. Biol. Chem. 2005, 280, 37107-37117, doi:10.1074/jbc.M504951200. 
116. Akira, S.; Takeda, K. Toll-like receptor signalling. Nat. Rev. Immunol. 2004, 4, 499511, doi:10.1038/nri1391.

117. Deguine, J.; Barton, G.M. MyD88: a central player in innate immune signaling. F1000Prime Rep. 2014, 6, 97, doi:10.12703/P6-97.

118. Yamamoto, M.; Sato, S.; Hemmi, H.; Sanjo, H.; Uematsu, S.; Kaisho, T.; Hoshino, K.; Takeuchi, O.; Kobayashi, M.; Fujita, T.; et al. Essential role for TIRAP in activation of the signalling cascade shared by TLR2 and TLR4. Nature 2002, 420, 324-329, doi:10.1038/nature01182.

119. Wesche, H.; Henzel, W.J.; Shillinglaw, W.; Li, S.; Cao, Z. MyD88: An Adapter That Recruits IRAK to the IL-1 Receptor Complex. Immunity 1997, 7, 837-847, doi:10.1016/S1074-7613(00)80402-1.

120. Yamashita, M.; Chattopadhyay, S.; Fensterl, V.; Zhang, Y.; Sen, G.C. A TRIFindependent branch of TLR3 signaling. J. Immunol. 2012, 188, 2825-2833, doi:10.4049/jimmunol.1103220.

121. Konno, H.; Yamamoto, T.; Yamazaki, K.; Gohda, J.; Akiyama, T.; Semba, K.; Goto, H.; Kato, A.; Yujiri, T.; Imai, T.; et al. TRAF6 establishes innate immune responses by activating NF-kappaB and IRF7 upon sensing cytosolic viral RNA and DNA. PLoS One 2009, 4, e5674, doi:10.1371/journal.pone.0005674.

122. Irie, T.; Muta, T.; Takeshige, K. TAK1 mediates an activation signal from toll-like receptor(s) to nuclear factor- $\kappa \mathrm{B}$ in lipopolysaccharide-stimulated macrophages. FEBS Letters 2000, 467, 160-164, doi:10.1016/S0014-5793(00)01146-7.

123. Yeretssian, G. Effector functions of NLRs in the intestine: innate sensing, cell death, and disease. Immunol. Res. 2012, 54, 25-36, doi:10.1007/s12026-012-8317-3.

124. Pellegrini, E.; Desfosses, A.; Wallmann, A.; Schulze, W.M.; Rehbein, K.; Mas, P.; Signor, L.; Gaudon, S.; Zenkeviciute, G.; Hons, M.; et al. RIP2 filament formation is required for NOD2 dependent NF- $\kappa$ B signalling. Nat. Commun. 2018, 9, 4043, doi:10.1038/s41467-018-06451-3.

125. McCarthy, J.V.; Ni, J.; Dixit, V.M. RIP2 is a novel NF-kappaB-activating and cell death-inducing kinase. J. Biol. Chem. 1998, 273, 16968-16975, doi:10.1074/jbc.273.27.16968.

126. Krieg, A.; Correa, R.G.; Garrison, J.B.; Le Negrate, G.; Welsh, K.; Huang, Z.; Knoefel, W.T.; Reed, J.C. XIAP mediates NOD signaling via interaction with RIP2. Proc. Natl. Acad. Sci. U. S. A. 2009, 106, 14524-14529, doi:10.1073/pnas.0907131106.

127. Bertrand, M.J.M.; Doiron, K.; Labbé, K.; Korneluk, R.G.; Barker, P.A.; Saleh, M. Cellular inhibitors of apoptosis cIAP1 and cIAP2 are required for innate immunity signaling by the pattern recognition receptors NOD1 and NOD2. Immunity 2009, 30, 789-801, doi:10.1016/j.immuni.2009.04.011. 
128. Hasegawa, M.; Fujimoto, Y.; Lucas, P.C.; Nakano, H.; Fukase, K.; Núñez, G.; Inohara, N. A critical role of RICK/RIP2 polyubiquitination in Nod-induced NFkappaB activation. EMBO J. 2008, 27, 373-383, doi:10.1038/sj.emboj.7601962.

129. Kato, H.; Takeuchi, O.; Mikamo-Satoh, E.; Hirai, R.; Kawai, T.; Matsushita, K.; Hiiragi, A.; Dermody, T.S.; Fujita, T.; Akira, S. Length-dependent recognition of double-stranded ribonucleic acids by retinoic acid-inducible gene-I and melanoma differentiation-associated gene 5. J. Exp. Med. 2008, 205, 1601-1610, doi:10.1084/jem.20080091.

130. Li, X.; Ranjith-Kumar, C.T.; Brooks, M.T.; Dharmaiah, S.; Herr, A.B.; Kao, C.; Li, P. The RIG-I-like receptor LGP2 recognizes the termini of double-stranded RNA. J. Biol. Chem. 2009, 284, 13881-13891, doi:10.1074/jbc.M900818200.

131. Kato, H.; Takeuchi, O.; Sato, S.; Yoneyama, M.; Yamamoto, M.; Matsui, K.; Uematsu, S.; Jung, A.; Kawai, T.; Ishii, K.J.; et al. Differential roles of MDA5 and RIG-I helicases in the recognition of RNA viruses. Nature 2006, 441, 101-105, doi:10.1038/nature04734.

132. Feng, Q.; Hato, S.V.; Langereis, M.A.; Zoll, J.; Virgen-Slane, R.; Peisley, A.; Hur, S.; Semler, B.L.; van Rij, R.P.; van Kuppeveld, F.J.M. MDA5 detects the doublestranded RNA replicative form in picornavirus-infected cells. Cell Rep. 2012, 2, 1187-1196, doi:10.1016/j.celrep.2012.10.005.

133. Takahasi, K.; Kumeta, H.; Tsuduki, N.; Narita, R.; Shigemoto, T.; Hirai, R.; Yoneyama, M.; Horiuchi, M.; Ogura, K.; Fujita, T.; et al. Solution structures of cytosolic RNA sensor MDA5 and LGP2 C-terminal domains: identification of the RNA recognition loop in RIG-I-like receptors. J. Biol. Chem. 2009, 284, 1746517474, doi:10.1074/jbc.M109.007179.

134. Meylan, E.; Curran, J.; Hofmann, K.; Moradpour, D.; Binder, M.; Bartenschlager, R.; Tschopp, J. Cardif is an adaptor protein in the RIG-I antiviral pathway and is targeted by hepatitis C virus. Nature 2005, 437, 1167-1172, doi:10.1038/nature04193.

135. Kawai, T.; Takahashi, K.; Sato, S.; Coban, C.; Kumar, H.; Kato, H.; Ishii, K.J.; Takeuchi, O.; Akira, S. IPS-1, an adaptor triggering RIG-I- and Mda5-mediated type I interferon induction. Nat. Immunol. 2005, 6, 981-988, doi:10.1038/ni1243.

136. Heaton, S.M.; Borg, N.A.; Dixit, V.M. Ubiquitin in the activation and attenuation of innate antiviral immunity. J. Exp. Med. 2016, 213, 1-13, doi:10.1084/jem.20151531.

137. Greenlund, A.C.; Schreiber, R.D.; Goeddel, D.V.; Pennica, D. Interferon-gamma induces receptor dimerization in solution and on cells. J. Biol. Chem. 1993, 268, 18103-18110.

138. Uzé, G.; Schreiber, G.; Piehler, J.; Pellegrini, S. The receptor of the type I interferon family. Curr. Top. Microbiol. Immunol. 2007, 316, 71-95, doi:10.1007/9783-540-71329-6_5. 
139. Silva, C.M.; Lu, H.; Weber, M.J.; Thorner, M.O. Differential tyrosine phosphorylation of JAK1, JAK2, and STAT1 by growth hormone and interferongamma in IM-9 cells. J. Biol. Chem. 1994, 269, 27532-27539.

140. Robertson, S.A.; Koleva, R.I.; Argetsinger, L.S.; Carter-Su, C.; Marto, J.A.; Feener, E.P.; Myers, M.G. Regulation of Jak2 function by phosphorylation of Tyr317 and Tyr637 during cytokine signaling. Mol. Cell. Biol. 2009, 29, 3367-3378, doi:10.1128/MCB.00278-09.

141. Gauzzi, M.C.; Velazquez, L.; McKendry, R.; Mogensen, K.E.; Fellous, M.; Pellegrini, S. Interferon-alpha-dependent activation of Tyk2 requires phosphorylation of positive regulatory tyrosines by another kinase. J. Biol. Chem. 1996, 271, 20494-20500, doi:10.1074/jbc.271.34.20494.

142. Rébé, C.; Végran, F.; Berger, H.; Ghiringhelli, F. STAT3 activation: A key factor in tumor immunoescape. JAKSTAT. 2013, 2, e23010, doi:10.4161/jkst.23010.

143.Sadzak, I.; Schiff, M.; Gattermeier, I.; Glinitzer, R.; Sauer, I.; Saalmüller, A.; Yang, E.; Schaljo, B.; Kovarik, P. Recruitment of Stat1 to chromatin is required for interferon-induced serine phosphorylation of Stat1 transactivation domain. Proc. Natl. Acad. Sci. U. S. A. 2008, 105, 8944-8949, doi:10.1073/pnas.0801794105.

144. Steen, H.C.; Gamero, A.M. STAT2 phosphorylation and signaling. JAKSTAT. 2013, 2, e25790, doi:10.4161/jkst.25790.

145. Varinou, L.; Ramsauer, K.; Karaghiosoff, M.; Kolbe, T.; Pfeffer, K.; Müller, M.; Decker, T. Phosphorylation of the Stat1 Transactivation Domain Is Required for Full-Fledged IFN- $\gamma$-Dependent Innate Immunity. Immunity 2003, 19, 793-802, doi:10.1016/S1074-7613(03)00322-4.

146. Zhang, K. Overview of Interferon: Characteristics, signaling and anti-cancer effect. Arch Biotechnol Biomed 2017, 1, 1-16, doi:10.29328/journal.hjb.1001001.

147. Sooryanarain, H.; Rogers, A.J.; Cao, D.; Haac, M.E.R.; Karpe, Y.A.; Meng, X.-J. ISG15 Modulates Type I Interferon Signaling and the Antiviral Response during Hepatitis E Virus Replication. J. Virol. 2017, 91, doi:10.1128/JVI.00621-17.

148. Kristiansen, H.; Scherer, C.A.; McVean, M.; Iadonato, S.P.; Vends, S.; Thavachelvam, K.; Steffensen, T.B.; Horan, K.A.; Kuri, T.; Weber, F.; et al. Extracellular 2'-5' oligoadenylate synthetase stimulates RNase L-independent antiviral activity: a novel mechanism of virus-induced innate immunity. J. Virol. 2010, 84, 11898-11904, doi:10.1128/JVI.01003-10.

149. Schneider, W.M.; Chevillotte, M.D.; Rice, C.M. Interferon-stimulated genes: a complex web of host defenses. Annu. Rev. Immunol. 2014, 32, 513-545, doi:10.1146/annurev-immunol-032713-120231.

150. Dauber, B.; Wolff, T. Activation of the Antiviral Kinase PKR and Viral Countermeasures. Viruses 2009, 1, 523-544, doi:10.3390/v1030523.

151. Murata, K.; Kang, J.-H.; Nagashima, S.; Matsui, T.; Karino, Y.; Yamamoto, Y.; Atarashi, T.; Oohara, M.; Uebayashi, M.; Sakata, H.; et al. IFN- $\lambda 3$ as a host 
immune response in acute hepatitis E virus infection. Cytokine 2020, 125, 154816, doi:10.1016/j.cyto.2019.154816.

152. Todt, D.; François, C.; Anggakusuma; Behrendt, P.; Engelmann, M.; Knegendorf, L.; Vieyres, G.; Wedemeyer, H.; Hartmann, R.; Pietschmann, T.; et al. Antiviral Activities of Different Interferon Types and Subtypes against Hepatitis E Virus Replication. Antimicrob. Agents Chemother. 2016, 60, 2132-2139, doi:10.1128/AAC.02427-15.

153. Wang, W.; Xu, L.; Brandsma, J.H.; Wang, Y.; Hakim, M.S.; Zhou, X.; Yin, Y.; Fuhler, G.M.; van der Laan, L.J.W.; van der Woude, C.J.; et al. Convergent Transcription of Interferon-stimulated Genes by TNF- $\alpha$ and IFN- $\alpha$ Augments Antiviral Activity against HCV and HEV. Sci. Rep. 2016, 6, 25482, doi:10.1038/srep25482.

154. Xu, L.; Zhou, X.; Wang, W.; Wang, Y.; Yin, Y.; van der Laan, L.J.W.; Sprengers, D.; Metselaar, H.J.; Peppelenbosch, M.P.; Pan, Q. IFN regulatory factor 1 restricts hepatitis E virus replication by activating STAT1 to induce antiviral IFNstimulated genes. FASEB J. 2016, 30, 3352-3367, doi:10.1096/fj.201600356R.

155. Devhare, P.B.; Chatterjee, S.N.; Arankalle, V.A.; Lole, K.S. Analysis of antiviral response in human epithelial cells infected with hepatitis E virus. PLoS One 2013, 8, e63793, doi:10.1371/journal.pone.0063793.

156. Devhare, P.B.; Desai, S.; Lole, K.S. Innate immune responses in human hepatocyte-derived cell lines alter genotype 1 hepatitis $E$ virus replication efficiencies. Sci. Rep. 2016, 6, 26827, doi:10.1038/srep26827.

157. Majumdar, M.; Ratho, R.K.; Chawla, Y.; Singh, M.P. Role of TLR gene expression and cytokine profiling in the immunopathogenesis of viral hepatitis E. J. Clin. Virol. 2015, 73, 8-13, doi:10.1016/j.jcv.2015.09.011.

158. Nan, Y.; Ma, Z.; Wang, R.; Yu, Y.; Kannan, H.; Fredericksen, B.; Zhang, Y.-J. Enhancement of interferon induction by ORF3 product of hepatitis E virus. J. Virol. 2014, 88, 8696-8705, doi:10.1128/JVI.01228-14.

159. Jagya, N.; Varma, S.P.K.; Thakral, D.; Joshi, P.; Durgapal, H.; Panda, S.K. RNAseq based transcriptome analysis of hepatitis E virus (HEV) and hepatitis B virus (HBV) replicon transfected Huh-7 cells. PLoS One 2014, 9, e87835, doi:10.1371/journal.pone.0087835.

160. Wang, W.; Wang, Y.; Qu, C.; Wang, S.; Zhou, J.; Cao, W.; Xu, L.; Ma, B.; Hakim, M.S.; Yin, Y.; et al. The RNA genome of hepatitis E virus robustly triggers an antiviral interferon response. Hepatology 2018, 67, 2096-2112, doi:10.1002/hep.29702.

161. Yin, X.; Li, X.; Ambardekar, C.; Hu, Z.; Lhomme, S.; Feng, Z. Hepatitis E virus persists in the presence of a type III interferon response. PLoS Pathog. 2017, 13, e1006417, doi:10.1371/journal.ppat.1006417. 
162. Sooryanarain, H.; Heffron, C.L.; Meng, X.-J. The U-Rich Untranslated Region of the Hepatitis E Virus Induces Differential Type I and Type III Interferon Responses in a Host Cell-Dependent Manner. mBio 2020, 11, doi:10.1128/mBio.03103-19.

163. Nan, Y.; Yu, Y.; Ma, Z.; Khattar, S.K.; Fredericksen, B.; Zhang, Y.-J. Hepatitis E virus inhibits type I interferon induction by ORF1 products. J. Virol. 2014, 88, 11924-11932, doi:10.1128/JVI.01935-14.

164. Bagdassarian, E.; Doceul, V.; Pellerin, M.; Demange, A.; Meyer, L.; Jouvenet, N.; Pavio, N. The Amino-Terminal Region of Hepatitis E Virus ORF1 Containing a Methyltransferase (Met) and a Papain-Like Cysteine Protease (PCP) Domain Counteracts Type I Interferon Response. Viruses 2018, 10, doi:10.3390/v10120726.

165. Surjit, M.; Varshney, B.; Lal, S.K. The ORF2 glycoprotein of hepatitis E virus inhibits cellular NF- $\kappa \mathrm{B}$ activity by blocking ubiquitination mediated proteasomal degradation of $\mathrm{I} \kappa \mathrm{B} \alpha$ in human hepatoma cells. BMC Biochem. 2012, 13, 7, doi:10.1186/1471-2091-13-7.

166. Dong, C.; Zafrullah, M.; Mixson-Hayden, T.; Dai, X.; Liang, J.; Meng, J.; Kamili, S. Suppression of interferon- $\alpha$ signaling by hepatitis E virus. Hepatology 2012, 55, 1324-1332, doi:10.1002/hep.25530.

167. Lei, Q.; Li, L.; Zhang, S.; Li, T.; Zhang, X.; Ding, X.; Qin, B. HEV ORF3 downregulates TLR7 to inhibit the generation of type I interferon via impairment of multiple signaling pathways. Sci. Rep. 2018, 8, 8585, doi:10.1038/s41598-01826975-4.

168. He, M.; Wang, M.; Huang, Y.; Peng, W.; Zheng, Z.; Xia, N.; Xu, J.; Tian, D. The ORF3 Protein of Genotype 1 Hepatitis E Virus Suppresses TLR3-induced NF- $\kappa$ B Signaling via TRADD and RIP1. Sci. Rep. 2016, 6, 27597, doi:10.1038/srep27597.

169. Li, Y.; Qu, C.; Yu, P.; Ou, X.; Pan, Q.; Wang, W. The Interplay between Host Innate Immunity and Hepatitis E Virus. Viruses 2019, 11, doi:10.3390/v11060541.

170. Olszewski, M.A.; Gray, J.; Vestal, D.J. In silico genomic analysis of the human and murine guanylate-binding protein (GBP) gene clusters. J. Interferon Cytokine Res. 2006, 26, 328-352, doi:10.1089/jir.2006.26.328.

171. Cheng, Y.S.; Colonno, R.J.; Yin, F.H. Interferon induction of fibroblast proteins with guanylate binding activity. Journal of Biological Chemistry 1983, 258, 77467750, doi:10.1016/S0021-9258(18)32242-7.

172. Lew, D.J.; Decker, T.; Strehlow, I.; Darnell, J.E. Overlapping elements in the guanylate-binding protein gene promoter mediate transcriptional induction by alpha and gamma interferons. Mol. Cell. Biol. 1991, 11, 182-191, doi:10.1128/mcb.11.1.182.

173. Kresse, A.; Konermann, C.; Degrandi, D.; Beuter-Gunia, C.; Wuerthner, J.; Pfeffer, K.; Beer, S. Analyses of murine GBP homology clusters based on in silico, 
in vitro and in vivo studies. BMC Genomics 2008, 9, 158, doi:10.1186/1471-2164-9158.

174. Cheng, Y.S.; Patterson, C.E.; Staeheli, P. Interferon-induced guanylate-binding proteins lack an $\mathrm{N}(\mathrm{T}) \mathrm{KXD}$ consensus motif and bind GMP in addition to GDP and GTP. Mol. Cell. Biol. 1991, 11, 4717-4725, doi:10.1128/MCB.11.9.4717.

175. Ghosh, A.; Praefcke, G.J.K.; Renault, L.; Wittinghofer, A.; Herrmann, C. How guanylate-binding proteins achieve assembly-stimulated processive cleavage of GTP to GMP. Nature 2006, 440, 101-104, doi:10.1038/nature04510.

176. Praefcke, G.J.; Geyer, M.; Schwemmle, M.; Robert Kalbitzer, H.; Herrmann, C. Nucleotide-binding characteristics of human guanylate-binding protein 1 (hGBP1) and identification of the third GTP-binding motif. J. Mol. Biol. 1999, 292, 321-332, doi:10.1006/jmbi.1999.3062.

177. Praefcke, G.J.K.; McMahon, H.T. The dynamin superfamily: universal membrane tubulation and fission molecules? Nat. Rev. Mol. Cell Biol. 2004, 5, 133-147, doi:10.1038/nrm1313.

178. Prakash, B.; Praefcke, G.J.; Renault, L.; Wittinghofer, A.; Herrmann, C. Structure of human guanylate-binding protein 1 representing a unique class of GTPbinding proteins. Nature 2000, 403, 567-571, doi:10.1038/35000617.

179. Schwemmle, M.; Staeheli, P. The interferon-induced 67-kDa guanylate-binding protein (hGBP1) is a GTPase that converts GTP to GMP. Journal of Biological Chemistry 1994, 269, 11299-11305, doi:10.1016/S0021-9258(19)78125-3.

180. Nantais, D.E.; Schwemmle, M.; Stickney, J.T.; Vestal, D.J.; Buss, J.E. Prenylation of an interferon-gamma-induced GTP-binding protein: the human guanylate binding protein, huGBP1. J. Leukoc. Biol. 1996, 60, 423-431, doi:10.1002/jlb.60.3.423.

181. Abdullah, N.; Srinivasan, B.; Modiano, N.; Cresswell, P.; Sau, A.K. Role of individual domains and identification of internal gap in human guanylate binding protein-1. J. Mol. Biol. 2009, 386, 690-703, doi:10.1016/j.jmb.2008.12.060.

182. Vöpel, T.; Syguda, A.; Britzen-Laurent, N.; Kunzelmann, S.; Lüdemann, M.-B.; Dovengerds, C.; Stürzl, M.; Herrmann, C. Mechanism of GTPase-activityinduced self-assembly of human guanylate binding protein 1. J. Mol. Biol. 2010, 400, 63-70, doi:10.1016/j.jmb.2010.04.053.

183. Praefcke, G.J.K.; Kloep, S.; Benscheid, U.; Lilie, H.; Prakash, B.; Herrmann, C. Identification of residues in the human guanylate-binding protein 1 critical for nucleotide binding and cooperative GTP hydrolysis. J. Mol. Biol. 2004, 344, 257269, doi:10.1016/j.jmb.2004.09.026.

184. Kunzelmann, S.; Praefcke, G.J.K.; Herrmann, C. Transient kinetic investigation of GTP hydrolysis catalyzed by interferon-gamma-induced hGBP1 (human guanylate binding protein 1). Journal of Biological Chemistry 2006, 281, 2862728635, doi:10.1074/jbc.M604911200. 
185. Shydlovskyi, S.; Zienert, A.Y.; Ince, S.; Dovengerds, C.; Hohendahl, A.; Dargazanli, J.M.; Blum, A.; Günther, S.D.; Kladt, N.; Stürzl, M.; et al. Nucleotidedependent farnesyl switch orchestrates polymerization and membrane binding of human guanylate-binding protein 1. Proc. Natl. Acad. Sci. U. S. A. 2017, 114, E5559-E5568, doi:10.1073/pnas.1620959114.

186. Schnoor, M.; Betanzos, A.; Weber, D.A.; Parkos, C.A. Guanylate-binding protein1 is expressed at tight junctions of intestinal epithelial cells in response to interferon-gamma and regulates barrier function through effects on apoptosis. Mucosal Immunol. 2009, 2, 33-42, doi:10.1038/mi.2008.62.

187. Tripal, P.; Bauer, M.; Naschberger, E.; Mörtinger, T.; Hohenadl, C.; Cornali, E.; Thurau, M.; Stürzl, M. Unique features of different members of the human guanylate-binding protein family. J. Interferon Cytokine Res. 2007, 27, 44-52, doi:10.1089/jir.2007.0086.

188. Britzen-Laurent, N.; Bauer, M.; Berton, V.; Fischer, N.; Syguda, A.; Reipschläger, S.; Naschberger, E.; Herrmann, C.; Stürzl, M. Intracellular trafficking of guanylate-binding proteins is regulated by heterodimerization in a hierarchical manner. PLoS One 2010, 5, e14246, doi:10.1371/journal.pone.0014246.

189. Kutsch, M.; Coers, J. Human guanylate binding proteins: nanomachines orchestrating host defense. FEBS J. 2020, doi:10.1111/febs.15662.

190. Nordmann, A.; Wixler, L.; Boergeling, Y.; Wixler, V.; Ludwig, S. A new splice variant of the human guanylate-binding protein 3 mediates anti-influenza activity through inhibition of viral transcription and replication. FASEB J. 2012, 26, 1290-1300, doi:10.1096/fj.11-189886.

191. Itsui, Y.; Sakamoto, N.; Kakinuma, S.; Nakagawa, M.; Sekine-Osajima, Y.; Tasaka-Fujita, M.; Nishimura-Sakurai, Y.; Suda, G.; Karakama, Y.; Mishima, K.; et al. Antiviral effects of the interferon-induced protein guanylate binding protein 1 and its interaction with the hepatitis C virus NS5B protein. Hepatology 2009, 50, 1727-1737, doi:10.1002/hep.23195.

192. Li, L.-F.; Yu, J.; Li, Y.; Wang, J.; Li, S.; Zhang, L.; Xia, S.-L.; Yang, Q.; Wang, X.; $\mathrm{Yu}$, S.; et al. Guanylate-Binding Protein 1, an Interferon-Induced GTPase, Exerts an Antiviral Activity against Classical Swine Fever Virus Depending on Its GTPase Activity. J. Virol. 2016, 90, 4412-4426, doi:10.1128/JVI.02718-15.

193. Pan, W.; Zuo, X.; Feng, T.; Shi, X.; Dai, J. Guanylate-binding protein 1 participates in cellular antiviral response to dengue virus. Virol. J. 2012, 9, 292, doi:10.1186/1743-422X-9-292.

194.Zou, Z.; Meng, Z.; Ma, C.; Liang, D.; Sun, R.; Lan, K. Guanylate-Binding Protein 1 Inhibits Nuclear Delivery of Kaposi's Sarcoma-Associated Herpesvirus Virions by Disrupting Formation of Actin Filament. J. Virol. 2017, 91, doi:10.1128/JVI.00632-17. 
195. Ostler, N.; Britzen-Laurent, N.; Liebl, A.; Naschberger, E.; Lochnit, G.; Ostler, M.; Forster, F.; Kunzelmann, P.; Ince, S.; Supper, V.; et al. Gamma interferon-induced guanylate binding protein 1 is a novel actin cytoskeleton remodeling factor. Mol. Cell. Biol. 2014, 34, 196-209, doi:10.1128/MCB.00664-13.

196. Biering, S.B.; Choi, J.; Halstrom, R.A.; Brown, H.M.; Beatty, W.L.; Lee, S.; McCune, B.T.; Dominici, E.; Williams, L.E.; Orchard, R.C.; et al. Viral Replication Complexes Are Targeted by LC3-Guided Interferon-Inducible GTPases. Cell Host Microbe 2017, 22, 74-85.e7, doi:10.1016/j.chom.2017.06.005.

197.Selleck, E.M.; Fentress, S.J.; Beatty, W.L.; Degrandi, D.; Pfeffer, K.; Virgin, H.W.; Macmicking, J.D.; Sibley, L.D. Guanylate-binding protein 1 (Gbp1) contributes to cell-autonomous immunity against Toxoplasma gondii. PLoS Pathog. 2013, 9, e1003320, doi:10.1371/journal.ppat.1003320.

198. Al-Zeer, M.A.; Al-Younes, H.M.; Lauster, D.; Abu Lubad, M.; Meyer, T.F. Autophagy restricts Chlamydia trachomatis growth in human macrophages via IFNG-inducible guanylate binding proteins. Autophagy 2013, 9, 50-62, doi:10.4161/auto.22482.

199. Kim, B.-H.; Shenoy, A.R.; Kumar, P.; Das, R.; Tiwari, S.; Macmicking, J.D. A family of IFN- $\gamma$-inducible $65-\mathrm{kD}$ GTPases protects against bacterial infection. Science 2011, 332, 717-721, doi:10.1126/science.1201711.

200.Steinman, R.M.; Brodie, S.E.; Cohn, Z.A. Membrane flow during pinocytosis. A stereologic analysis. J. Cell Biol. 1976, 68, 665-687, doi:10.1083/jcb.68.3.665.

201.Steinman, R.M.; Mellman, I.S.; Muller, W.A.; Cohn, Z.A. Endocytosis and the recycling of plasma membrane. J. Cell Biol. 1983, 96, 1-27, doi:10.1083/jcb.96.1.1.

202. Burd, C.; Cullen, P.J. Retromer: a master conductor of endosome sorting. Cold Spring Harb. Perspect. Biol. 2014, 6, doi:10.1101/cshperspect.a016774.

203. Nagano, M.; Toshima, J.Y.; Siekhaus, D.E.; Toshima, J. Rab5-mediated endosome formation is regulated at the trans-Golgi network. Commun. Biol. 2019, 2, 419, doi:10.1038/s42003-019-0670-5.

204.Stoorvogel, W.; Strous, G.J.; Geuze, H.J.; Oorschot, V.; Schwartzt, A.L. Late endosomes derive from early endosomes by maturation. Cell 1991, 65, 417-427, doi:10.1016/0092-8674(91)90459-C.

205. Xie, S.; Bahl, K.; Reinecke, J.B.; Hammond, G.R.V.; Naslavsky, N.; Caplan, S. The endocytic recycling compartment maintains cargo segregation acquired upon exit from the sorting endosome. Mol. Biol. Cell 2016, 27, 108-126, doi:10.1091/mbc.E15-07-0514.

206. Eaton, S.; Martin-Belmonte, F. Cargo sorting in the endocytic pathway: a key regulator of cell polarity and tissue dynamics. Cold Spring Harb. Perspect. Biol. 2014, 6, a016899, doi:10.1101/cshperspect.a016899.

207.Schmidt, O.; Teis, D. The ESCRT machinery. Curr. Biol. 2012, 22, R116-20, doi:10.1016/j.cub.2012.01.028. 
208. Mullock, B.M.; Bright, N.A.; Fearon, C.W.; Gray, S.R.; Luzio, J.P. Fusion of lysosomes with late endosomes produces a hybrid organelle of intermediate density and is NSF dependent. J. Cell Biol. 1998, 140, 591-601, doi:10.1083/jcb.140.3.591.

209. Huotari, J.; Helenius, A. Endosome maturation. EMBO J. 2011, 30, 3481-3500, doi:10.1038/emboj.2011.286.

210. Pereira-Leal, J.B.; Hume, A.N.; Seabra, M.C. Prenylation of Rab GTPases: molecular mechanisms and involvement in genetic disease. FEBS Letters 2001, 498, 197-200, doi:10.1016/s0014-5793(01)02483-8.

211. Alexandrov, K.; Horiuchi, H.; Steele-Mortimer, O.; Seabra, M.C.; Zerial, M. Rab escort protein-1 is a multifunctional protein that accompanies newly prenylated rab proteins to their target membranes. EMBO J. 1994, 13, 5262-5273.

212. Barr, F.; Lambright, D.G. Rab GEFs and GAPs. Curr. Opin. Cell Biol. 2010, 22, 461470, doi:10.1016/j.ceb.2010.04.007.

213. Seabra, M.C.; Mules, E.H.; Hume, A.N. Rab GTPases, intracellular traffic and disease. Trends Mol. Med. 2002, 8, 23-30, doi:10.1016/s1471-4914(01)02227-4.

214. Horiuchi, H.; Lippé, R.; McBride, H.M.; Rubino, M.; Woodman, P.; Stenmark, H.; Rybin, V.; Wilm, M.; Ashman, K.; Mann, M.; et al. A novel Rab5 GDP/GTP exchange factor complexed to Rabaptin-5 links nucleotide exchange to effector recruitment and function. Cell 1997, 90, 1149-1159, doi:10.1016/s00928674(00)80380-3.

215. Stenmark, H.; Vitale, G.; Ullrich, O.; Zerial, M. Rabaptin-5 is a direct effector of the small GTPase Rab5 in endocytic membrane fusion. Cell 1995, 83, 423-432, doi:10.1016/0092-8674(95)90120-5.

216. Futter, C.E.; Collinson, L.M.; Backer, J.M.; Hopkins, C.R. Human VPS34 is required for internal vesicle formation within multivesicular endosomes. J. Cell Biol. 2001, 155, 1251-1264, doi:10.1083/jcb.200108152.

217. Lawe, D.C.; Patki, V.; Heller-Harrison, R.; Lambright, D.; Corvera, S. The FYVE domain of early endosome antigen 1 is required for both phosphatidylinositol 3phosphate and Rab5 binding. Critical role of this dual interaction for endosomal localization. Journal of Biological Chemistry 2000, 275, 3699-3705, doi:10.1074/jbc.275.5.3699.

218. Merithew, E.; Stone, C.; Eathiraj, S.; Lambright, D.G. Determinants of Rab5 interaction with the $\mathrm{N}$ terminus of early endosome antigen 1. Journal of Biological Chemistry 2003, 278, 8494-8500, doi:10.1074/jbc.M211514200.

219. Nielsen, E.; Christoforidis, S.; Uttenweiler-Joseph, S.; Miaczynska, M.; Dewitte, F.; Wilm, M.; Hoflack, B.; Zerial, M. Rabenosyn-5, a novel Rab5 effector, is complexed with hVPS45 and recruited to endosomes through a FYVE finger domain. J. Cell Biol. 2000, 151, 601-612, doi:10.1083/jcb.151.3.601. 
220. Grosshans, B.L.; Ortiz, D.; Novick, P. Rabs and their effectors: achieving specificity in membrane traffic. Proc. Natl. Acad. Sci. U. S. A. 2006, 103, 1182111827, doi:10.1073/pnas.0601617103.

221. Jean, S.; Kiger, A.A. Coordination between RAB GTPase and phosphoinositide regulation and functions. Nat. Rev. Mol. Cell Biol. 2012, 13, 463-470, doi:10.1038/nrm3379.

222. Kouranti, I.; Sachse, M.; Arouche, N.; Goud, B.; Echard, A. Rab35 regulates an endocytic recycling pathway essential for the terminal steps of cytokinesis. Curr. Biol. 2006, 16, 1719-1725, doi:10.1016/j.cub.2006.07.020.

223. McCaffrey, M.W.; Bielli, A.; Cantalupo, G.; Mora, S.; Roberti, V.; Santillo, M.; Drummond, F.; Bucci, C. Rab4 affects both recycling and degradative endosomal trafficking. FEBS Letters 2001, 495, 21-30, doi:10.1016/S0014-5793(01)02359-6.

224. Vanlandingham, P.A.; Ceresa, B.P. Rab7 regulates late endocytic trafficking downstream of multivesicular body biogenesis and cargo sequestration. Journal of Biological Chemistry 2009, 284, 12110-12124, doi:10.1074/jbc.M809277200.

225. Vieira, O.V.; Bucci, C.; Harrison, R.E.; Trimble, W.S.; Lanzetti, L.; Gruenberg, J.; Schreiber, A.D.; Stahl, P.D.; Grinstein, S. Modulation of Rab5 and Rab7 recruitment to phagosomes by phosphatidylinositol 3-kinase. Mol. Cell. Biol. 2003, 23, 2501-2514, doi:10.1128/MCB.23.7.2501-2514.2003.

226. Horgan, C.P.; Oleksy, A.; Zhdanov, A.V.; Lall, P.Y.; White, I.J.; Khan, A.R.; Futter, C.E.; McCaffrey, J.G.; McCaffrey, M.W. Rab11-FIP3 is critical for the structural integrity of the endosomal recycling compartment. Traffic 2007, 8, 414430, doi:10.1111/j.1600-0854.2007.00543.x.

227. Campa, C.C.; Margaria, J.P.; Derle, A.; Del Giudice, M.; Santis, M.C. de; Gozzelino, L.; Copperi, F.; Bosia, C.; Hirsch, E. Rab11 activity and PtdIns(3)P turnover removes recycling cargo from endosomes. Nat. Chem. Biol. 2018, 14, 801-810, doi:10.1038/s41589-018-0086-4.

228. Ang, A.L.; Taguchi, T.; Francis, S.; Fölsch, H.; Murrells, L.J.; Pypaert, M.; Warren, G.; Mellman, I. Recycling endosomes can serve as intermediates during transport from the Golgi to the plasma membrane of MDCK cells. J. Cell Biol. 2004, 167, 531-543, doi:10.1083/jcb.200408165.

229. Kucera, A.; Bakke, O.; Progida, C. The multiple roles of Rab9 in the endolysosomal system. Commun. Integr. Biol. 2016, 9, e1204498, doi:10.1080/19420889.2016.1204498.

230. Mallard, F.; Tang, B.L.; Galli, T.; Tenza, D.; Saint-Pol, A.; Yue, X.; Antony, C.; Hong, W.; Goud, B.; Johannes, L. Early/recycling endosomes-to-TGN transport involves two SNARE complexes and a Rab6 isoform. J. Cell Biol. 2002, 156, 653664, doi:10.1083/jcb.200110081. 
231. Chavrier, P.; Parton, R.G.; Hauri, H.P.; Simons, K.; Zerial, M. Localization of low molecular weight GTP binding proteins to exocytic and endocytic compartments. Cell 1990, 62, 317-329, doi:10.1016/0092-8674(90)90369-p.

232. Nuoffer, C.; Davidson, H.W.; Matteson, J.; Meinkoth, J.; Balch, W.E. A GDPbound of rab1 inhibits protein export from the endoplasmic reticulum and transport between Golgi compartments. J. Cell Biol. 1994, 125, 225-237, doi:10.1083/jcb.125.2.225.

233. Hammer, J.A.; Wu, X.S. Rabs grab motors: defining the connections between Rab GTPases and motor proteins. Curr. Opin. Cell Biol. 2002, 14, 69-75, doi:10.1016/S0955-0674(01)00296-4.

234. Müller, S.; Dennemärker, J.; Reinheckel, T. Specific functions of lysosomal proteases in endocytic and autophagic pathways. Biochim. Biophys. Acta 2012, 1824, 34-43, doi:10.1016/j.bbapap.2011.07.003.

235. Vale-Costa, S.; Amorim, M.J. Recycling Endosomes and Viral Infection. Viruses 2016, 8, 64, doi:10.3390/v8030064.

236. Piper, R.C.; Katzmann, D.J. Biogenesis and function of multivesicular bodies. Annu. Rev. Cell Dev. Biol. 2007, 23, 519-547, doi:10.1146/annurev.cellbio.23.090506.123319.

237. Frankel, E.B.; Audhya, A. ESCRT-dependent cargo sorting at multivesicular endosomes. Semin. Cell Dev. Biol. 2018, 74, 4-10, doi:10.1016/j.semcdb.2017.08.020.

238. Mosesso, N.; Nagel, M.-K.; Isono, E. Ubiquitin recognition in endocytic trafficking - with or without ESCRT-0. J. Cell Sci. 2019, 132, doi:10.1242/jcs.232868.

239. Komada, M.; Soriano, P. Hrs, a FYVE finger protein localized to early endosomes, is implicated in vesicular traffic and required for ventral folding morphogenesis. Genes Dev. 1999, 13, 1475-1485, doi:10.1101/gad.13.11.1475.

240. Edgar, J.R.; Eden, E.R.; Futter, C.E. Hrs- and CD63-dependent competing mechanisms make different sized endosomal intraluminal vesicles. Traffic 2014, 15, 197-211, doi:10.1111/tra.12139.

241. Teo, H.; Gill, D.J.; Sun, J.; Perisic, O.; Veprintsev, D.B.; Vallis, Y.; Emr, S.D.; Williams, R.L. ESCRT-I core and ESCRT-II GLUE domain structures reveal role for GLUE in linking to ESCRT-I and membranes. Cell 2006, 125, 99-111, doi:10.1016/j.cell.2006.01.047.

242.Sundquist, W.I.; Schubert, H.L.; Kelly, B.N.; Hill, G.C.; Holton, J.M.; Hill, C.P. Ubiquitin recognition by the human TSG101 protein. Mol. Cell 2004, 13, 783-789, doi:10.1016/s1097-2765(04)00129-7.

243. Teo, H.; Veprintsev, D.B.; Williams, R.L. Structural insights into endosomal sorting complex required for transport (ESCRT-I) recognition of ubiquitinated proteins. Journal of Biological Chemistry 2004, 279, 28689-28696, doi:10.1074/jbc.M400023200. 
244. Boura, E.; Ivanov, V.; Carlson, L.-A.; Mizuuchi, K.; Hurley, J.H. Endosomal sorting complex required for transport (ESCRT) complexes induce phaseseparated microdomains in supported lipid bilayers. J. Biol. Chem. 2012, 287, 28144-28151, doi:10.1074/jbc.M112.378646.

245. Babst, M.; Katzmann, D.J.; Estepa-Sabal, E.J.; Meerloo, T.; Emr, S.D. Escrt-III: an endosome-associated heterooligomeric protein complex required for mvb sorting. Dev. Cell 2002, 3, 271-282, doi:10.1016/s1534-5807(02)00220-4.

246. Babst, M.; Katzmann, D.J.; Snyder, W.B.; Wendland, B.; Emr, S.D. Endosomeassociated complex, ESCRT-II, recruits transport machinery for protein sorting at the multivesicular body. Dev. Cell 2002, 3, 283-289, doi:10.1016/s15345807(02)00219-8.

247. Sachse, M.; Strous, G.J.; Klumperman, J. ATPase-deficient hVPS4 impairs formation of internal endosomal vesicles and stabilizes bilayered clathrin coats on endosomal vacuoles. J. Cell Sci. 2004, 117, 1699-1708, doi:10.1242/jcs.00998.

248. Wemmer, M.; Azmi, I.; West, M.; Davies, B.; Katzmann, D.; Odorizzi, G. Bro1 binding to Snf7 regulates ESCRT-III membrane scission activity in yeast. J. Cell Biol. 2011, 192, 295-306, doi:10.1083/jcb.201007018.

249. Rodriguez-Furlan, C.; Minina, E.A.; Hicks, G.R. Remove, Recycle, Degrade: Regulating Plasma Membrane Protein Accumulation. Plant Cell 2019, 31, 28332854, doi:10.1105/tpc.19.00433.

250. Dores, M.R.; Chen, B.; Lin, H.; Soh, U.J.K.; Paing, M.M.; Montagne, W.A.; Meerloo, T.; Trejo, J. ALIX binds a YPX(3)L motif of the GPCR PAR1 and mediates ubiquitin-independent ESCRT-III/MVB sorting. J. Cell Biol. 2012, 197, 407-419, doi:10.1083/jcb.201110031.

251. Yamashita, Y.; Kojima, K.; Tsukahara, T.; Agawa, H.; Yamada, K.; Amano, Y.; Kurotori, N.; Tanaka, N.; Sugamura, K.; Takeshita, T. Ubiquitin-independent binding of Hrs mediates endosomal sorting of the interleukin-2 receptor betachain. J. Cell Sci. 2008, 121, 1727-1738, doi:10.1242/jcs.024455.

252. MacDonald, C.; Payne, J.A.; Aboian, M.; Smith, W.; Katzmann, D.J.; Piper, R.C. A family of tetraspans organizes cargo for sorting into multivesicular bodies. Dev. Cell 2015, 33, 328-342, doi:10.1016/j.devcel.2015.03.007.

253. Wubbolts, R.; Leckie, R.S.; Veenhuizen, P.T.M.; Schwarzmann, G.; Möbius, W.; Hoernschemeyer, J.; Slot, J.-W.; Geuze, H.J.; Stoorvogel, W. Proteomic and biochemical analyses of human B cell-derived exosomes. Potential implications for their function and multivesicular body formation. Journal of Biological Chemistry 2003, 278, 10963-10972, doi:10.1074/jbc.M207550200.

254. Valadi, H.; Ekström, K.; Bossios, A.; Sjöstrand, M.; Lee, J.J.; Lötvall, J.O. Exosome-mediated transfer of mRNAs and microRNAs is a novel mechanism of genetic exchange between cells. Nat. Cell Biol. 2007, 9, 654-659, doi:10.1038/ncb1596. 
255. Irion, U.; St Johnston, D. bicoid RNA localization requires specific binding of an endosomal sorting complex. Nature 2007, 445, 554-558, doi:10.1038/nature05503.

256. Pornillos, O.; Higginson, D.S.; Stray, K.M.; Fisher, R.D.; Garrus, J.E.; Payne, M.; He, G.-P.; Wang, H.E.; Morham, S.G.; Sundquist, W.I. HIV Gag mimics the Tsg101-recruiting activity of the human Hrs protein. J. Cell Biol. 2003, 162, 425434, doi:10.1083/jcb.200302138.

257. Garrus, J.E.; Schwedler, U.K. von; Pornillos, O.W.; Morham, S.G.; Zavitz, K.H.; Wang, H.E.; Wettstein, D.A.; Stray, K.M.; Côté, M.; Rich, R.L.; et al. Tsg101 and the Vacuolar Protein Sorting Pathway Are Essential for HIV-1 Budding. Cell 2001, 107, 55-65, doi:10.1016/S0092-8674(01)00506-2.

258. Johnstone, R.M.; Adam, M.; Hammond, J.R.; Orr, L.; Turbide, C. Vesicle formation during reticulocyte maturation. Association of plasma membrane activities with released vesicles (exosomes). Journal of Biological Chemistry 1987, 262, 9412-9420.

259. Wei, H.; Chen, Q.; Lin, L.; Sha, C.; Li, T.; Liu, Y.; Yin, X.; Xu, Y.; Chen, L.; Gao, W.; et al. Regulation of exosome production and cargo sorting. Int. J. Biol. Sci. 2021, 17, 163-177, doi:10.7150/ijbs.53671.

260. Capelli, N.; Marion, O.; Dubois, M.; Allart, S.; Bertrand-Michel, J.; Lhomme, S.; Abravanel, F.; Izopet, J.; Chapuy-Regaud, S. Vectorial Release of Hepatitis E Virus in Polarized Human Hepatocytes. J. Virol. 2019, 93, doi:10.1128/JVI.0120718.

261. Chapuy-Regaud, S.; Dubois, M.; Plisson-Chastang, C.; Bonnefois, T.; Lhomme, S.; Bertrand-Michel, J.; You, B.; Simoneau, S.; Gleizes, P.-E.; Flan, B.; et al. Characterization of the lipid envelope of exosome encapsulated HEV particles protected from the immune response. Biochimie 2017, 141, 70-79, doi:10.1016/j.biochi.2017.05.003.

262. Wißing, M.H.; Brüggemann, Y.; Steinmann, E.; Todt, D. Virus-Host Cell Interplay during Hepatitis E Virus Infection. Trends Microbiol. 2020, doi:10.1016/j.tim.2020.07.002.

263. Oechslin, N.; Moradpour, D.; Gouttenoire, J. On the Host Side of the Hepatitis E Virus Life Cycle. Cells 2020, 9, doi:10.3390/cells9051294.

264. Evans, W.H.; Hardison, W.G. Phospholipid, cholesterol, polypeptide and glycoprotein composition of hepatic endosome subfractions. Biochem. J. 1985, 232, 33-36, doi:10.1042/bj2320033.

265. Chen, Z.; Rand, R.P. The influence of cholesterol on phospholipid membrane curvature and bending elasticity. Biophys. J. 1997, 73, 267-276, doi:10.1016/S00063495(97)78067-6.

266. Churchward, M.A.; Rogasevskaia, T.; Höfgen, J.; Bau, J.; Coorssen, J.R. Cholesterol facilitates the native mechanism of Ca2+-triggered membrane fusion. J. Cell Sci. 2005, 118, 4833-4848, doi:10.1242/jcs.02601. 
267. Meyer, F. de; Smit, B. Effect of cholesterol on the structure of a phospholipid bilayer. Proc. Natl. Acad. Sci. U. S. A. 2009, 106, 3654-3658, doi:10.1073/pnas.0809959106.

268. Cooper, R.A. Influence of increased membrane cholesterol on membrane fluidity and cell function in human red blood cells. J. Supramol. Struct. 1978, 8, 413-430, doi:10.1002/jss.400080404.

269. Lorent, J.H.; Diaz-Rohrer, B.; Lin, X.; Spring, K.; Gorfe, A.A.; Levental, K.R.; Levental, I. Structural determinants and functional consequences of protein affinity for membrane rafts. Nat. Commun. 2017, 8, 1219, doi:10.1038/s41467-01701328-3.

270.Sobo, K.; Chevallier, J.; Parton, R.G.; Gruenberg, J.; van der Goot, F.G. Diversity of raft-like domains in late endosomes. PLoS One 2007, 2, e391, doi:10.1371/journal.pone.0000391.

271. Hanzal-Bayer, M.F.; Hancock, J.F. Lipid rafts and membrane traffic. FEBS Letters 2007, 581, 2098-2104, doi:10.1016/j.febslet.2007.03.019.

272. Silvius, J.R. Role of cholesterol in lipid raft formation: lessons from lipid model systems. Biochimica et Biophysica Acta (BBA) - Biomembranes 2003, 1610, 174-183, doi:10.1016/S0005-2736(03)00016-6.

273. Lee, Y.K.; Yu, E.-S.; Ahn, D.J.; Ryu, Y.-S. Elasticity-Driven Membrane Budding through Cholesterol Concentration on Supported Lipid Monolayer-Bilayer Junction. Adv. Mater. Interfaces 2020, 7, 2000937, doi:10.1002/admi.202000937.

274. Bissig, C.; Gruenberg, J. Lipid sorting and multivesicular endosome biogenesis. Cold Spring Harb. Perspect. Biol. 2013, 5, a016816, doi:10.1101/cshperspect.a016816.

275. Kobayashi, T.; Beuchat, M.-H.; Chevallier, J.; Makino, A.; Mayran, N.; Escola, J.M.; Lebrand, C.; Cosson, P.; Kobayashi, T.; Gruenberg, J. Separation and characterization of late endosomal membrane domains. Journal of Biological Chemistry 2002, 277, 32157-32164, doi:10.1074/jbc.M202838200.

276. Cuesta-Geijo, M.Á.; Chiappi, M.; Galindo, I.; Barrado-Gil, L.; Muñoz-Moreno, R.; Carrascosa, J.L.; Alonso, C. Cholesterol Flux Is Required for Endosomal Progression of African Swine Fever Virions during the Initial Establishment of Infection. J. Virol. 2016, 90, 1534-1543, doi:10.1128/JVI.02694-15.

277. Reverter, M.; Rentero, C.; Muga, S.V. de; Alvarez-Guaita, A.; Mulay, V.; Cairns, R.; Wood, P.; Monastyrskaya, K.; Pol, A.; Tebar, F.; et al. Cholesterol transport from late endosomes to the Golgi regulates t-SNARE trafficking, assembly, and function. MBoC 2011, 22, 4108-4123, doi:10.1091/mbc.e11-04-0332r.

278. Enrich, C.; Rentero, C.; Hierro, A.; Grewal, T. Role of cholesterol in SNAREmediated trafficking on intracellular membranes. J. Cell Sci. 2015, 128, 1071-1081, doi:10.1242/jcs.164459.

279. Lang, T.; Bruns, D.; Wenzel, D.; Riedel, D.; Holroyd, P.; Thiele, C.; Jahn, R. SNAREs are concentrated in cholesterol-dependent clusters that define docking 
and fusion sites for exocytosis. EMBO J. 2001, 20, 2202-2213,

doi:10.1093/emboj/20.9.2202.

280. Milovanovic, D.; Honigmann, A.; Koike, S.; Göttfert, F.; Pähler, G.; Junius, M.; Müllar, S.; Diederichsen, U.; Janshoff, A.; Grubmüller, H.; et al. Hydrophobic mismatch sorts SNARE proteins into distinct membrane domains. Nat. Commun. 2015, 6, 5984, doi:10.1038/ncomms6984.

281.Stratton, B.S.; Warner, J.M.; Wu, Z.; Nikolaus, J.; Wei, G.; Wagnon, E.; Baddeley, D.; Karatekin, E.; O'Shaughnessy, B. Cholesterol Increases the Openness of SNARE-Mediated Flickering Fusion Pores. Biophys. J. 2016, 110, 1538-1550, doi:10.1016/j.bpj.2016.02.019.

282. Johansson, M.; Lehto, M.; Tanhuanpää, K.; Cover, T.L.; Olkkonen, V.M. The oxysterol-binding protein homologue ORP1L interacts with Rab7 and alters functional properties of late endocytic compartments. MBoC 2005, 16, 5480-5492, doi:10.1091/mbc.e05-03-0189.

283. Progida, C.; Malerød, L.; Stuffers, S.; Brech, A.; Bucci, C.; Stenmark, H. RILP is required for the proper morphology and function of late endosomes. J. Cell Sci. 2007, 120, 3729-3737, doi:10.1242/jcs.017301.

284. Rocha, N.; Kuijl, C.; van der Kant, R.; Janssen, L.; Houben, D.; Janssen, H.; Zwart, W.; Neefjes, J. Cholesterol sensor ORP1L contacts the ER protein VAP to control Rab7-RILP-p150 Glued and late endosome positioning. J. Cell Biol. 2009, 185, 1209-1225, doi:10.1083/jcb.200811005.

285. Thakur, S.; Relich, P.K.; Sorokina, E.M.; Gyparaki, M.T.; Lakadamyali, M. ORP1L regulates dynein clustering on endolysosmal membranes in response to cholesterol levels, 2020.

286. Jordens, I.; Fernandez-Borja, M.; Marsman, M.; Dusseljee, S.; Janssen, L.; Calafat, J.; Janssen, H.; Wubbolts, R.; Neefjes, J. The Rab7 effector protein RILP controls lysosomal transport by inducing the recruitment of dynein-dynactin motors. Curr. Biol. 2001, 11, 1680-1685, doi:10.1016/s0960-9822(01)00531-0.

287. Russell, D.W. Nuclear orphan receptors control cholesterol catabolism. Cell 1999, 97, 539-542, doi:10.1016/s0092-8674(00)80763-1.

288. Edwards, P.A.; Ericsson, J. Sterols and isoprenoids: signaling molecules derived from the cholesterol biosynthetic pathway. Annu. Rev. Biochem. 1999, 68, 157-185, doi:10.1146/annurev.biochem.68.1.157.

289. Shimomura, I.; Bashmakov, Y.; Shimano, H.; Horton, J.D.; Goldstein, J.L.; Brown, M.S. Cholesterol feeding reduces nuclear forms of sterol regulatory element binding proteins in hamster liver. Proc. Natl. Acad. Sci. U. S. A. 1997, 94, 1235412359, doi:10.1073/pnas.94.23.12354.

290. Geelen, M.J.; Gibson, D.M.; Rodwell, V.W. Hydroxymethylglutaryl-CoA reductase--the rate-limiting enzyme of cholesterol biosynthesis. A report of a 
meeting held at Nijenrode Castle, Breukelen, The Netherlands, August 24, 1985. FEBS Letters 1986, 201, 183-186, doi:10.1016/0014-5793(86)80604-4.

291. Jurczyluk, J.; Munoz, M.A.; Skinner, O.P.; Chai, R.C.; Ali, N.; Palendira, U.; Quinn, J.M.; Preston, A.; Tangye, S.G.; Brown, A.J.; et al. Mevalonate kinase deficiency leads to decreased prenylation of Rab GTPases. Immunol. Cell Biol. 2016, 94, 994-999, doi:10.1038/icb.2016.58.

292. Chen, H.; Yang, J.; Low, P.S.; Cheng, J.-X. Cholesterol level regulates endosome motility via Rab proteins. Biophys. J. 2008, 94, 1508-1520, doi:10.1529/biophysj.106.099366.

293. Sobo, K.; Le Blanc, I.; Luyet, P.-P.; Fivaz, M.; Ferguson, C.; Parton, R.G.; Gruenberg, J.; van der Goot, F.G. Late endosomal cholesterol accumulation leads to impaired intra-endosomal trafficking. PLoS One 2007, 2, e851, doi:10.1371/journal.pone.0000851.

294. LINDGREN, F.T.; ELLIOTT, H.A.; GOFMAN, J.W. The ultracentrifugal characterization and isolation of human blood lipids and lipoproteins, with applications to the study of atherosclerosis. J. Phys. Colloid Chem. 1951, 55, 80-93, doi:10.1021/j150484a010.

295. PEDERSEN, K.O. On a low-density lipoprotein appearing in normal human plasma. J. Phys. Colloid Chem. 1947, 51, 156-163, doi:10.1021/j150451a011.

296. Anderson, R.G.; Brown, M.S.; Goldstein, J.L. Role of the coated endocytic vesicle in the uptake of receptor-bound low density lipoprotein in human fibroblasts. Cell 1977, 10, 351-364, doi:10.1016/0092-8674(77)90022-8.

297. Basu, S.K.; Goldstein, J.L.; Anderson, R.G.W.; Brown, M.S. Monensin interrupts the recycling of low density lipoprotein receptors in human fibroblasts. Cell 1981, 24, 493-502, doi:10.1016/0092-8674(81)90340-8.

298. Brown, M.S.; Anderson, R.G.W.; Goldstein, J.L. Recycling receptors: The roundtrip itinerary of migrant membrane proteins. Cell 1983, 32, 663-667, doi:10.1016/0092-8674(83)90052-1.

299. Leren, T.P. Sorting an LDL receptor with bound PCSK9 to intracellular degradation. Atherosclerosis 2014, 237, 76-81, doi:10.1016/j.atherosclerosis.2014.08.038.

300. Warner, T.G.; Dambach, L.M.; Shin, J.H.; O'Brien, J.S. Purification of the lysosomal acid lipase from human liver and its role in lysosomal lipid hydrolysis. Journal of Biological Chemistry 1981, 256, 2952-2957.

301. Li, J.; Pfeffer, S.R. Lysosomal membrane glycoproteins bind cholesterol and contribute to lysosomal cholesterol export. Elife 2016, 5, doi:10.7554/eLife.21635.

302. Locatelli-Hoops, S.; Remmel, N.; Klingenstein, R.; Breiden, B.; Rossocha, M.; Schoeniger, M.; Koenigs, C.; Saenger, W.; Sandhoff, K. Saposin A mobilizes lipids from low cholesterol and high bis(monoacylglycerol)phosphate-containing 
membranes: patient variant Saposin A lacks lipid extraction capacity. Journal of Biological Chemistry 2006, 281, 32451-32460, doi:10.1074/jbc.M607281200.

303. Heybrock, S.; Kanerva, K.; Meng, Y.; Ing, C.; Liang, A.; Xiong, Z.-J.; Weng, X.; Ah Kim, Y.; Collins, R.; Trimble, W.; et al. Lysosomal integral membrane protein-2 (LIMP-2/SCARB2) is involved in lysosomal cholesterol export. Nat. Commun. 2019, 10, 3521, doi:10.1038/s41467-019-11425-0.

304. Infante, R.E.; Wang, M.L.; Radhakrishnan, A.; Kwon, H.J.; Brown, M.S.; Goldstein, J.L. NPC2 facilitates bidirectional transfer of cholesterol between NPC1 and lipid bilayers, a step in cholesterol egress from lysosomes. Proc. Natl. Acad. Sci. U. S. A. 2008, 105, 15287-15292, doi:10.1073/pnas.0807328105.

305. Watari, H.; Blanchette-Mackie, E.J.; Dwyer, N.K.; Glick, J.M.; Patel, S.; Neufeld, E.B.; Brady, R.O.; Pentchev, P.G.; Strauss, J.F. Niemann-Pick C1 protein: obligatory roles for N-terminal domains and lysosomal targeting in cholesterol mobilization. Proc. Natl. Acad. Sci. U. S. A. 1999, 96, 805-810, doi:10.1073/pnas.96.3.805.

306. Chu, B.-B.; Liao, Y.-C.; Qi, W.; Xie, C.; Du, X.; Wang, J.; Yang, H.; Miao, H.-H.; Li, B.-L.; Song, B.-L. Cholesterol transport through lysosome-peroxisome membrane contacts. Cell 2015, 161, 291-306, doi:10.1016/j.cell.2015.02.019.

307. Daniele, T.; Hurbain, I.; Vago, R.; Casari, G.; Raposo, G.; Tacchetti, C.; Schiaffino, M.V. Mitochondria and melanosomes establish physical contacts modulated by Mfn2 and involved in organelle biogenesis. Curr. Biol. 2014, 24, 393-403, doi:10.1016/j.cub.2014.01.007.

308. Muñoz-Braceras, S.; Tornero-Écija, A.R.; Vincent, O.; Escalante, R. VPS13A is closely associated with mitochondria and is required for efficient lysosomal degradation. Dis. Model. Mech. 2019, 12, doi:10.1242/dmm.036681.

309. Höglinger, D.; Burgoyne, T.; Sanchez-Heras, E.; Hartwig, P.; Colaco, A.; Newton, J.; Futter, C.E.; Spiegel, S.; Platt, F.M.; Eden, E.R. NPC1 regulates ER contacts with endocytic organelles to mediate cholesterol egress. Nat. Commun. 2019, 10, 4276, doi:10.1038/s41467-019-12152-2.

310. Olzmann, J.A.; Carvalho, P. Dynamics and functions of lipid droplets. Nat. Rev. Mol. Cell Biol. 2019, 20, 137-155, doi:10.1038/s41580-018-0085-z.

311. Meng, Y.; Heybrock, S.; Neculai, D.; Saftig, P. Cholesterol Handling in Lysosomes and Beyond. Trends Cell Biol. 2020, 30, 452-466, doi:10.1016/j.tcb.2020.02.007.

312. Yang, T.; Espenshade, P.J.; Wright, M.E.; Yabe, D.; Gong, Y.; Aebersold, R.; Goldstein, J.L.; Brown, M.S. Crucial step in cholesterol homeostasis: sterols promote binding of SCAP to INSIG-1, a membrane protein that facilitates retention of SREBPs in ER. Cell 2002, 110, 489-500, doi:10.1016/s00928674(02)00872-3. 
313.Sun, L.-P.; Seemann, J.; Goldstein, J.L.; Brown, M.S. Sterol-regulated transport of SREBPs from endoplasmic reticulum to Golgi: Insig renders sorting signal in Scap inaccessible to COPII proteins. Proc. Natl. Acad. Sci. U. S. A. 2007, 104, 65196526, doi:10.1073/pnas.0700907104.

314. Rawson, R.B.; DeBose-Boyd, R.; Goldstein, J.L.; Brown, M.S. Failure to cleave sterol regulatory element-binding proteins (SREBPs) causes cholesterol auxotrophy in Chinese hamster ovary cells with genetic absence of SREBP cleavage-activating protein. Journal of Biological Chemistry 1999, 274, 28549-28556, doi:10.1074/jbc.274.40.28549.

315. Ozbay, T.; Rowan, A.; Leon, A.; Patel, P.; Sewer, M.B. Cyclic adenosine 5'monophosphate-dependent sphingosine-1-phosphate biosynthesis induces human CYP17 gene transcription by activating cleavage of sterol regulatory element binding protein 1. Endocrinology 2006, 147, 1427-1437, doi:10.1210/en.2005-1091.

316. Wang, X.; Sato, R.; Brown, M.S.; Hua, X.; Goldstein, J.L. SREBP-1, a membranebound transcription factor released by sterol-regulated proteolysis. Cell 1994, 77, 53-62, doi:10.1016/0092-8674(94)90234-8.

317. Yokoyama, C.; Wang, X.; Briggs, M.R.; Admon, A.; Wu, J.; Hua, X.; Goldstein, J.L.; Brown, M.S. SREBP-1, a basic-helix-loop-helix-leucine zipper protein that controls transcription of the low density lipoprotein receptor gene. Cell 1993, 75, 187-197.

318. Brown, M.S.; Goldstein, J.L. The SREBP pathway: regulation of cholesterol metabolism by proteolysis of a membrane-bound transcription factor. Cell 1997, 89, 331-340, doi:10.1016/s0092-8674(00)80213-5.

319. Fernández-Alvarez, A.; Alvarez, M.S.; Gonzalez, R.; Cucarella, C.; Muntané, J.; Casado, M. Human SREBP1c expression in liver is directly regulated by peroxisome proliferator-activated receptor alpha (PPARalpha). J. Biol. Chem. 2011, 286, 21466-21477, doi:10.1074/jbc.M110.209973.

320. Kalaany, N.Y.; Mangelsdorf, D.J. LXRS and FXR: the yin and yang of cholesterol and fat metabolism. Annu. Rev. Physiol. 2006, 68, 159-191, doi:10.1146/annurev.physiol.68.033104.152158.

321. Xu, Y.; Li, F.; Zalzala, M.; Xu, J.; Gonzalez, F.J.; Adorini, L.; Lee, Y.-K.; Yin, L.; $Z$ hang, $Y$. Farnesoid $X$ receptor activation increases reverse cholesterol transport by modulating bile acid composition and cholesterol absorption in mice. Hepatology 2016, 64, 1072-1085, doi:10.1002/hep.28712.

322. Zhao, C.; Dahlman-Wright, K. Liver X receptor in cholesterol metabolism. J. Endocrinol. 2010, 204, 233-240, doi:10.1677/JOE-09-0271.

323. Murthy, S.; Born, E.; Mathur, S.N.; Field, F.J. LXR/RXR activation enhances basolateral efflux of cholesterol in CaCo-2 cells. J. Lipid Res. 2002, 43, 1054-1064, doi:10.1194/jlr.m100358-jlr200. 
324. Peet, D.J.; Turley, S.D.; Ma, W.; Janowski, B.A.; Lobaccaro, J.M.; Hammer, R.E.; Mangelsdorf, D.J. Cholesterol and bile acid metabolism are impaired in mice lacking the nuclear oxysterol receptor LXR alpha. Cell 1998, 93, 693-704, doi:10.1016/s0092-8674(00)81432-4.

325. Willy, P.J.; Umesono, K.; Ong, E.S.; Evans, R.M.; Heyman, R.A.; Mangelsdorf, D.J. LXR, a nuclear receptor that defines a distinct retinoid response pathway. Genes Dev. 1995, 9, 1033-1045, doi:10.1101/gad.9.9.1033.

326. Yue, L.; Ye, F.; Gui, C.; Luo, H.; Cai, J.; Shen, J.; Chen, K.; Shen, X.; Jiang, H. Ligand-binding regulation of LXR/RXR and LXR/PPAR heterodimerizations: SPR technology-based kinetic analysis correlated with molecular dynamics simulation. Protein Sci. 2005, 14, 812-822, doi:10.1110/ps.04951405.

327.Son, Y.L.; Park, O.G.; Kim, G.S.; Lee, J.W.; Lee, Y.C. RXR heterodimerization allosterically activates LXR binding to the second NR box of activating signal cointegrator-2. Biochem. J. 2008, 410, 319-330, doi:10.1042/BJ20070837.

328. Rühl, R.; Krzyżosiak, A.; Niewiadomska-Cimicka, A.; Rochel, N.; Szeles, L.; Vaz, B.; Wietrzych-Schindler, M.; Álvarez, S.; Szklenar, M.; Nagy, L.; et al. 9-cis-13,14Dihydroretinoic Acid Is an Endogenous Retinoid Acting as RXR Ligand in Mice. PLoS Genet. 2015, 11, e1005213, doi:10.1371/journal.pgen.1005213.

329. Janowski, B.A.; Willy, P.J.; Devi, T.R.; Falck, J.R.; Mangelsdorf, D.J. An oxysterol signalling pathway mediated by the nuclear receptor LXR alpha. Nature 1996, 383, 728-731, doi:10.1038/383728a0.

330. Forman, B.M.; Ruan, B.; Chen, J.; Schroepfer, G.J.; Evans, R.M. The orphan nuclear receptor LXRalpha is positively and negatively regulated by distinct products of mevalonate metabolism. Proc. Natl. Acad. Sci. U. S. A. 1997, 94, 10588-10593, doi:10.1073/pnas.94.20.10588.

331. Boadu, E.; Nelson, R.C.; Francis, G.A. ABCA1-dependent mobilization of lysosomal cholesterol requires functional Niemann-Pick C2 but not NiemannPick C1 protein. Biochim. Biophys. Acta 2012, 1821, 396-404, doi:10.1016/j.bbalip.2011.11.013.

332. Boadu, E.; Francis, G.A. The role of vesicular transport in ABCA1-dependent lipid efflux and its connection with NPC pathways. J. Mol. Med. (Berl) 2006, 84, 266-275, doi:10.1007/s00109-005-0001-9.

333.Zhao, G.-J.; Yin, K.; Fu, Y.-C.; Tang, C.-K. The interaction of ApoA-I and ABCA1 triggers signal transduction pathways to mediate efflux of cellular lipids. Mol. Med. 2012, 18, 149-158, doi:10.2119/molmed.2011.00183.

334. Gelissen, I.C.; Harris, M.; Rye, K.-A.; Quinn, C.; Brown, A.J.; Kockx, M.; Cartland, S.; Packianathan, M.; Kritharides, L.; Jessup, W. ABCA1 and ABCG1 synergize to mediate cholesterol export to apoA-I. Arterioscler. Thromb. Vasc. Biol. 2006, 26, 534-540, doi:10.1161/01.ATV.0000200082.58536.e1. 
335. Yu, X.-H.; Jiang, N.; Yao, P.-B.; Zheng, X.-L.; Cayabyab, F.S.; Tang, C.-K. NPC1, intracellular cholesterol trafficking and atherosclerosis. Clin. Chim. Acta 2014, 429, 69-75, doi:10.1016/j.cca.2013.11.026.

336. Chen, L.; Kwong, M.; Lu, R.; Ginzinger, D.; Lee, C.; Leung, L.; Chan, J.Y. Nrf1 is critical for redox balance and survival of liver cells during development. Mol. Cell. Biol. 2003, 23, 4673-4686, doi:10.1128/MCB.23.13.4673-4686.2003.

337. Leung, L.; Kwong, M.; Hou, S.; Lee, C.; Chan, J.Y. Deficiency of the Nrf1 and Nrf2 transcription factors results in early embryonic lethality and severe oxidative stress. Journal of Biological Chemistry 2003, 278, 48021-48029, doi:10.1074/jbc.M308439200.

338. Widenmaier, S.B.; Snyder, N.A.; Nguyen, T.B.; Arduini, A.; Lee, G.Y.; Arruda, A.P.; Saksi, J.; Bartelt, A.; Hotamisligil, G.S. NRF1 Is an ER Membrane Sensor that Is Central to Cholesterol Homeostasis. Cell 2017, 171, 1094-1109.e15, doi:10.1016/j.cell.2017.10.003.

339. Steffen, J.; Seeger, M.; Koch, A.; Krüger, E. Proteasomal degradation is transcriptionally controlled by TCF11 via an ERAD-dependent feedback loop. Mol. Cell 2010, 40, 147-158, doi:10.1016/j.molcel.2010.09.012.

340. Radhakrishnan, S.K.; den Besten, W.; Deshaies, R.J. p97-dependent retrotranslocation and proteolytic processing govern formation of active Nrf1 upon proteasome inhibition. Elife 2014, 3, e01856, doi:10.7554/eLife.01856.

341. La Roche, M. de; Hamilton, C.; Mortensen, R.; Jeyaprakash, A.A.; Ghosh, S.; Anand, P.K. Trafficking of cholesterol to the ER is required for NLRP3 inflammasome activation. J. Cell Biol. 2018, 217, 3560-3576, doi:10.1083/jcb.201709057.

342. Kelley, N.; Jeltema, D.; Duan, Y.; He, Y. The NLRP3 Inflammasome: An Overview of Mechanisms of Activation and Regulation. Int. J. Mol. Sci. 2019, 20, doi:10.3390/ijms20133328.

343. Seol, W.; Choi, H.S.; Moore, D.D. Isolation of proteins that interact specifically with the retinoid $X$ receptor: two novel orphan receptors. Mol. Endocrinol. 1995, 9, 72-85, doi:10.1210/mend.9.1.7760852.

344. Kassam, A.; Miao, B.; Young, P.R.; Mukherjee, R. Retinoid X receptor (RXR) agonist-induced antagonism of farnesoid $X$ receptor (FXR) activity due to absence of coactivator recruitment and decreased DNA binding. Journal of Biological Chemistry 2003, 278, 10028-10032, doi:10.1074/jbc.M208312200.

345. Forman, B.M.; Goode, E.; Chen, J.; Oro, A.E.; Bradley, D.J.; Perlmann, T.; Noonan, D.J.; Burka, L.T.; McMorris, T.; Lamph, W.W.; et al. Identification of a nuclear receptor that is activated by farnesol metabolites. Cell 1995, 81, 687-693, doi:10.1016/0092-8674(95)90530-8. 
346. Crick, D.C.; Andres, D.A.; Waechter, C.J. Farnesol is utilized for protein isoprenylation and the biosynthesis of cholesterol in mammalian cells. Biochem. Biophys. Res. Commun. 1995, 211, 590-599, doi:10.1006/bbrc.1995.1854.

347.Mi, L.-Z.; Devarakonda, S.; Harp, J.M.; Han, Q.; Pellicciari, R.; Willson, T.M.; Khorasanizadeh, S.; Rastinejad, F. Structural basis for bile acid binding and activation of the nuclear receptor FXR. Mol. Cell 2003, 11, 1093-1100, doi:10.1016/s1097-2765(03)00112-6.

348. Tu, H.; Okamoto, A.Y.; Shan, B. FXR, a bile acid receptor and biological sensor. Trends Cardiovasc. Med. 2000, 10, 30-35, doi:10.1016/s1050-1738(00)00043-8.

349. Eloranta, J.J.; Kullak-Ublick, G.A. The role of FXR in disorders of bile acid homeostasis. Physiology (Bethesda) 2008, 23, 286-295, doi:10.1152/physiol.00020.2008.

350. Pineda Torra, I.; Claudel, T.; Duval, C.; Kosykh, V.; Fruchart, J.-C.; Staels, B. Bile acids induce the expression of the human peroxisome proliferator-activated receptor alpha gene via activation of the farnesoid X receptor. Mol. Endocrinol. 2003, 17, 259-272, doi:10.1210/me.2002-0120.

351.Zhang, Y.; Lickteig, A.J.; Csanaky, I.L.; Klaassen, C.D. Activation of PPAR $\alpha$ decreases bile acids in livers of female mice while maintaining bile flow and biliary bile acid excretion. Toxicol. Appl. Pharmacol. 2018, 338, 112-123, doi:10.1016/j.taap.2017.11.014.

352. Gupta, S.; Pandak, W.M.; Hylemon, P.B. LXR alpha is the dominant regulator of CYP7A1 transcription. Biochem. Biophys. Res. Commun. 2002, 293, 338-343, doi:10.1016/S0006-291X(02)00229-2.

353.Szanto, A.; Benko, S.; Szatmari, I.; Balint, B.L.; Furtos, I.; Rühl, R.; Molnar, S.; Csiba, L.; Garuti, R.; Calandra, S.; et al. Transcriptional regulation of human CYP27 integrates retinoid, peroxisome proliferator-activated receptor, and liver $\mathrm{X}$ receptor signaling in macrophages. Mol. Cell. Biol. 2004, 24, 8154-8166, doi:10.1128/MCB.24.18.8154-8166.2004.

354. Noshiro, M.; Okuda, K. Molecular cloning and sequence analysis of cDNA encoding human cholesterol 7 alpha-hydroxylase. FEBS Letters 1990, 268, 137140, doi:10.1016/0014-5793(90)80992-r.

355. Li, T.; Chanda, D.; Zhang, Y.; Choi, H.-S.; Chiang, J.Y.L. Glucose stimulates cholesterol 7alpha-hydroxylase gene transcription in human hepatocytes. J. Lipid Res. 2010, 51, 832-842, doi:10.1194/jlr.M002782.

356. Bodin, K.; Andersson, U.; Rystedt, E.; Ellis, E.; Norlin, M.; Pikuleva, I.; Eggertsen, G.; Björkhem, I.; Diczfalusy, U. Metabolism of 4 beta -hydroxycholesterol in humans. Journal of Biological Chemistry 2002, 277, 31534-31540, doi:10.1074/jbc.M201712200. 
357. Cali, J.J.; Russell, D.W. Characterization of human sterol 27-hydroxylase. A mitochondrial cytochrome P-450 that catalyzes multiple oxidation reaction in bile acid biosynthesis. Journal of Biological Chemistry 1991, 266, 7774-7778.

358. Pikuleva, I.A.; Babiker, A.; Waterman, M.R.; Björkhem, I. Activities of recombinant human cytochrome P450c27 (CYP27) which produce intermediates of alternative bile acid biosynthetic pathways. Journal of Biological Chemistry 1998, 273, 18153-18160, doi:10.1074/jbc.273.29.18153.

359. Pandak, W.M.; Ren, S.; Marques, D.; Hall, E.; Redford, K.; Mallonee, D.; Bohdan, P.; Heuman, D.; Gil, G.; Hylemon, P. Transport of cholesterol into mitochondria is rate-limiting for bile acid synthesis via the alternative pathway in primary rat hepatocytes. Journal of Biological Chemistry 2002, 277, 48158-48164, doi:10.1074/jbc.M205244200.

360. Ferdinandusse, S.; Houten, S.M. Peroxisomes and bile acid biosynthesis. Biochim. Biophys. Acta 2006, 1763, 1427-1440, doi:10.1016/j.bbamcr.2006.09.001.

361. Davis, R.A.; Elliott, T.S.; Lattier, G.R.; Showalter, R.B.; Kern, F. Regulation of bile acid synthesis via direct effects on the microsomal membrane. Biochemistry 1986, 25, 1632-1636, doi:10.1021/bi00355a028.

362. Chiang, J.Y.L. Negative feedback regulation of bile acid metabolism: impact on liver metabolism and diseases. Hepatology 2015, 62, 1315-1317, doi:10.1002/hep.27964.

363. Falany, C.N.; Johnson, M.R.; Barnes, S.; Diasio, R.B. Glycine and taurine conjugation of bile acids by a single enzyme. Molecular cloning and expression of human liver bile acid CoA:amino acid N-acyltransferase. Journal of Biological Chemistry 1994, 269, 19375-19379.

364. Yabuuchi, H.; Tanaka, K.; Maeda, M.; Takemura, M.; Oka, M.; Ohashi, R.; Tamai, I. Cloning of the dog bile salt export pump (BSEP; ABCB11) and functional comparison with the human and rat proteins. Biopharm. Drug Dispos. 2008, 29, 441-448, doi:10.1002/bdd.629.

365. Paulusma, C.C.; Waart, D.R. de; Kunne, C.; Mok, K.S.; Elferink, R.P.J.O. Activity of the bile salt export pump (ABCB11) is critically dependent on canalicular membrane cholesterol content. Journal of Biological Chemistry 2009, 284, 99479954, doi:10.1074/jbc.M808667200.

366. Hayashi, H.; Inamura, K.; Aida, K.; Naoi, S.; Horikawa, R.; Nagasaka, H.; Takatani, T.; Fukushima, T.; Hattori, A.; Yabuki, T.; et al. AP2 adaptor complex mediates bile salt export pump internalization and modulates its hepatocanalicular expression and transport function. Hepatology 2012, 55, 18891900, doi:10.1002/hep.25591.

367. Hayashi, H.; Takada, T.; Suzuki, H.; Onuki, R.; Hofmann, A.F.; Sugiyama, Y. Transport by vesicles of glycine- and taurine-conjugated bile salts and 
taurolithocholate 3-sulfate: a comparison of human BSEP with rat Bsep. Biochim. Biophys. Acta 2005, 1738, 54-62, doi:10.1016/j.bbalip.2005.10.006.

368. Ballatori, N.; Christian, W.V.; Lee, J.Y.; Dawson, P.A.; Soroka, C.J.; Boyer, J.L.; Madejczyk, M.S.; Li, N. OSTalpha-OSTbeta: a major basolateral bile acid and steroid transporter in human intestinal, renal, and biliary epithelia. Hepatology 2005, 42, 1270-1279, doi:10.1002/hep.20961.

369. Deng, R.; Yang, D.; Yang, J.; Yan, B. Oxysterol 22(R)-hydroxycholesterol induces the expression of the bile salt export pump through nuclear receptor farsenoid $X$ receptor but not liver $X$ receptor. J. Pharmacol. Exp. Ther. 2006, 317, 317-325, doi:10.1124/jpet.105.097758.

370. Okuwaki, M.; Takada, T.; Iwayanagi, Y.; Koh, S.; Kariya, Y.; Fujii, H.; Suzuki, H. LXR alpha transactivates mouse organic solute transporter alpha and beta via IR1 elements shared with FXR. Pharm. Res. 2007, 24, 390-398, doi:10.1007/s11095006-9163-6.

371. Landrier, J.-F.; Eloranta, J.J.; Vavricka, S.R.; Kullak-Ublick, G.A. The nuclear receptor for bile acids, FXR, transactivates human organic solute transporteralpha and -beta genes. Am. J. Physiol. Gastrointest. Liver Physiol. 2006, 290, G47685, doi:10.1152/ajpgi.00430.2005.

372. Ananthanarayanan, M.; Balasubramanian, N.; Makishima, M.; Mangelsdorf, D.J.; Suchy, F.J. Human bile salt export pump promoter is transactivated by the farnesoid X receptor/bile acid receptor. Journal of Biological Chemistry 2001, 276, 28857-28865, doi:10.1074/jbc.M011610200.

373. Knight, B.L.; Hebbachi, A.; Hauton, D.; Brown, A.-M.; Wiggins, D.; Patel, D.D.; Gibbons, G.F. A role for PPARalpha in the control of SREBP activity and lipid synthesis in the liver. Biochem. J. 2005, 389, 413-421, doi:10.1042/BJ20041896.

374. Miyata, M.; Hata, T.; Yamazoe, Y.; Yoshinari, K. SREBP-2 negatively regulates FXR-dependent transcription of FGF19 in human intestinal cells. Biochem. Biophys. Res. Commun. 2014, 443, 477-482, doi:10.1016/j.bbrc.2013.11.126.

375.Wong, J.; Quinn, C.M.; Brown, A.J. SREBP-2 positively regulates transcription of the cholesterol efflux gene, ABCA1, by generating oxysterol ligands for LXR. Biochem. J. 2006, 400, 485-491, doi:10.1042/BJ20060914.

376. THORP, J.M.; WARING, W.S. Modification of metabolism and distribution of lipids by ethyl chlorophenoxyisobutyrate. Nature 1962, 194, 948-949, doi:10.1038/194948a0.

377. Brown, W.V. Potential use of fenofibrate and other fibric acid derivatives in the clinic. Am. J. Med. 1987, 83, 85-89, doi:10.1016/0002-9343(87)90876-x.

378. Rodney, G.; Uhlendorf, P.; Maxwell, R.E. The hypolipidaemic effect of gemfibrozil (CI-719) in laboratory animals. Proc. R. Soc. Med. 1976, 69 Suppl 2, 610. 
379. Post, S.M.; Duez, H.; Gervois, P.P.; Staels, B.; Kuipers, F.; Princen, H.M. Fibrates suppress bile acid synthesis via peroxisome proliferator-activated receptoralpha-mediated downregulation of cholesterol 7alpha-hydroxylase and sterol 27hydroxylase expression. Arterioscler. Thromb. Vasc. Biol. 2001, 21, 1840-1845, doi:10.1161/hq1101.098228.

380. Elisaf, M. Effects of fibrates on serum metabolic parameters. Curr. Med. Res. Opin. 2002, 18, 269-276, doi:10.1185/030079902125000516.

381. Endo, A. The discovery and development of HMG-CoA reductase inhibitors. J. Lipid Res. 1992, 33, 1569-1582.

382. Randomised trial of cholesterol lowering in 4444 patients with coronary heart disease: the Scandinavian Simvastatin Survival Study (4S). Lancet 1994, 344, 1383-1389.

383. Day, A.P.; Bellavia, S.; Jones, O.T.; Stansbie, D. Effect of simvastatin therapy on cell membrane cholesterol content and membrane function as assessed by polymorphonuclear cell NADPH oxidase activity. Ann. Clin. Biochem. 1997, 34 (Pt 3), 269-275, doi:10.1177/000456329703400308.

384. Tanaka, H.; Ohtsuka, I.; Kogushi, M.; Kimura, T.; Fujimori, T.; Saeki, T.; Hayashi, K.; Kobayashi, H.; Yamada, T.; Hiyoshi, H.; et al. Effect of the acylCoA:cholesterol acyltransferase inhibitor, E5324, on experimental atherosclerosis in rabbits. Atherosclerosis 1994, 107, 187-201, doi:10.1016/0021-9150(94)90020-5.

385. Delsing, D.J.; Offerman, E.H.; van Duyvenvoorde, W.; van der Boom, H.; Wit, E.C. de; Gijbels, M.J.; van der Laarse, A.; Jukema, J.W.; Havekes, L.M.; Princen, H.M. Acyl-CoA:cholesterol acyltransferase inhibitor avasimibe reduces atherosclerosis in addition to its cholesterol-lowering effect in ApoE*3-Leiden mice. Circulation 2001, 103, 1778-1786, doi:10.1161/01.cir.103.13.1778.

386. Friche, E.; Jensen, P.B.; Nissen, N.I. Comparison of cyclosporin A and SDZ PSC833 as multidrug-resistance modulators in a daunorubicin-resistant Ehrlich ascites tumor. Cancer Chemother. Pharmacol. 1992, 30, 235-237, doi:10.1007/BF00686321.

387. Nagao, K.; Maeda, M.; Mañucat, N.B.; Ueda, K. Cyclosporine A and PSC833 inhibit ABCA1 function via direct binding. Biochim. Biophys. Acta 2013, 1831, 398406, doi:10.1016/j.bbalip.2012.11.002.

388. Hirschfield, G.M.; Chazouillères, O.; Drenth, J.P.; Thorburn, D.; Harrison, S.A.; Landis, C.S.; Mayo, M.J.; Muir, A.J.; Trotter, J.F.; Leeming, D.J.; et al. Effect of NGM282, an FGF19 analogue, in primary sclerosing cholangitis: A multicenter, randomized, double-blind, placebo-controlled phase II trial. J. Hepatol. 2019, 70, 483-493, doi:10.1016/j.jhep.2018.10.035.

389. Harrison, S.A.; Rinella, M.E.; Abdelmalek, M.F.; Trotter, J.F.; Paredes, A.H.; Arnold, H.L.; Kugelmas, M.; Bashir, M.R.; Jaros, M.J.; Ling, L.; et al. NGM282 for treatment of non-alcoholic steatohepatitis: a multicentre, randomised, double- 
blind, placebo-controlled, phase 2 trial. Lancet 2018, 391, 1174-1185, doi:10.1016/S0140-6736(18)30474-4.

390.Zhou, M.; Learned, R.M.; Rossi, S.J.; Tian, H.; DePaoli, A.M.; Ling, L. Therapeutic FGF19 promotes HDL biogenesis and transhepatic cholesterol efflux to prevent atherosclerosis. J. Lipid Res. 2019, 60, 550-565, doi:10.1194/jlr.M089961.

391. Chaudhary, R.; Garg, J.; Shah, N.; Sumner, A. PCSK9 inhibitors: A new era of lipid lowering therapy. World J. Cardiol. 2017, 9, 76-91, doi:10.4330/wjc.v9.i2.76. 392. Sabatine, M.S.; Giugliano, R.P.; Wiviott, S.D.; Raal, F.J.; Blom, D.J.; Robinson, J.; Ballantyne, C.M.; Somaratne, R.; Legg, J.; Wasserman, S.M.; et al. Efficacy and safety of evolocumab in reducing lipids and cardiovascular events. N. Engl. J. Med. 2015, 372, 1500-1509, doi:10.1056/NEJMoa1500858.

393. Stein, E.A.; Mellis, S.; Yancopoulos, G.D.; Stahl, N.; Logan, D.; Smith, W.B.; Lisbon, E.; Gutierrez, M.; Webb, C.; Wu, R.; et al. Effect of a monoclonal antibody to PCSK9 on LDL cholesterol. N. Engl. J. Med. 2012, 366, 1108-1118, doi:10.1056/NEJMoa1105803.

394. Giard, D.J.; Aaronson, S.A.; Todaro, G.J.; Arnstein, P.; Kersey, J.H.; Dosik, H.; Parks, W.P. In vitro cultivation of human tumors: establishment of cell lines derived from a series of solid tumors. J. Natl. Cancer Inst. 1973, 51, 1417-1423, doi:10.1093/jnci/51.5.1417.

395. Schemmerer, M.; Apelt, S.; Trojnar, E.; Ulrich, R.G.; Wenzel, J.J.; Johne, R. Enhanced Replication of Hepatitis E Virus Strain 47832c in an A549-Derived Subclonal Cell Line. Viruses 2016, 8, doi:10.3390/v8100267.

396. Li, M.; Mukasa, A.; Inda, M.d.-M.; Zhang, J.; Chin, L.; Cavenee, W.; Furnari, F. Guanylate binding protein 1 is a novel effector of EGFR-driven invasion in glioblastoma. J. Exp. Med. 2011, 208, 2657-2673, doi:10.1084/jem.20111102.

397. Konno, T.; Takano, K.; Kaneko, Y.; Kakuki, T.; Nomura, K.; Yajima, R.; Kakiuchi, A.; Kohno, T.; Himi, T.; Kojima, T. Guanylate binding protein-1-mediated epithelial barrier in human salivary gland duct epithelium. Exp. Cell Res. 2018, 371, 31-41, doi:10.1016/j.yexcr.2018.07.033.

398. Tabari, D.; Scholl, C.; Steffens, M.; Weickhardt, S.; Elgner, F.; Bender, D.; Herrlein, M.-L.; Sabino, C.; Semkova, V.; Peitz, M.; et al. Impact of Zika Virus Infection on Human Neural Stem Cell MicroRNA Signatures. Viruses 2020, 12, doi:10.3390/v12111219.

399. Schindelin, J.; Arganda-Carreras, I.; Frise, E.; Kaynig, V.; Longair, M.; Pietzsch, T.; Preibisch, S.; Rueden, C.; Saalfeld, S.; Schmid, B.; et al. Fiji: an open-source platform for biological-image analysis. Nat. Methods 2012, 9, 676-682, doi:10.1038/nmeth.2019.

400. Ramakrishnan, M.A. Determination of $50 \%$ endpoint titer using a simple formula. World J. Virol. 2016, 5, 85-86, doi:10.5501/wjv.v5.i2.85. 
401. Pei, G.; Schnettger, L.; Bronietzki, M.; Repnik, U.; Griffiths, G.; Gutierrez, M.G. Interferon- $\gamma$-inducible Rab20 regulates endosomal morphology and EGFR degradation in macrophages. Mol. Biol. Cell 2015, 26, 3061-3070, doi:10.1091/mbc.E14-11-1547.

402. Zhang, H.; Zoued, A.; Liu, X.; Sit, B.; Waldor, M.K. Functional remodeling of lysosomes by type I interferon modifies host defense, 2020.

403. Kobuna, H.; Inoue, T.; Shibata, M.; Gengyo-Ando, K.; Yamamoto, A.; Mitani, S.; Arai, H. Multivesicular body formation requires OSBP-related proteins and cholesterol. PLoS Genet. 2010, 6, doi:10.1371/journal.pgen.1001055.

404. Taniguchi, T.; Takaoka, A. The interferon-alpha/beta system in antiviral responses: a multimodal machinery of gene regulation by the IRF family of transcription factors. Curr. Opin. Immunol. 2002, 14, 111-116, doi:10.1016/s09527915(01)00305-3.

405. Schoenborn, J.R.; Wilson, C.B. Regulation of interferon-gamma during innate and adaptive immune responses. Adv. Immunol. 2007, 96, 41-101, doi:10.1016/S0065-2776(07)96002-2.

406. Naschberger, E.; Werner, T.; Vicente, A.B.; Guenzi, E.; Töpolt, K.; Leubert, R.; Lubeseder-Martellato, C.; Nelson, P.J.; Stürzl, M. Nuclear factor-kappaB motif and interferon-alpha-stimulated response element co-operate in the activation of guanylate-binding protein-1 expression by inflammatory cytokines in endothelial cells. Biochem. J. 2004, 379, 409-420, doi:10.1042/BJ20031873.

407. Hingane, S.; Joshi, N.; Surjit, M.; Ranjith-Kumar, C.T. Hepatitis E Virus ORF2 Inhibits RIG-I Mediated Interferon Response. Front. Microbiol. 2020, 11, 656, doi:10.3389/fmicb.2020.00656.

408. Haller, O.; Kochs, G.; Weber, F. The interferon response circuit: induction and suppression by pathogenic viruses. Virology 2006, 344, 119-130, doi:10.1016/j.virol.2005.09.024.

409. Dong, B.; Xu, L.; Zhou, A.; Hassel, B.A.; Lee, X.; Torrence, P.F.; Silverman, R.H. Intrinsic molecular activities of the interferon-induced 2-5A-dependent RNase. Journal of Biological Chemistry 1994, 269, 14153-14158.

410. Qiu, X.; Guo, H.; Yang, J.; Ji, Y.; Wu, C.-S.; Chen, X. Down-regulation of guanylate binding protein 1 causes mitochondrial dysfunction and cellular senescence in macrophages. Sci. Rep. 2018, 8, 1679, doi:10.1038/s41598-018-198287.

411.Zhou, X.; Wang, Y.; Metselaar, H.J.; Janssen, H.L.A.; Peppelenbosch, M.P.; Pan, Q. Rapamycin and everolimus facilitate hepatitis $\mathrm{E}$ virus replication: revealing a basal defense mechanism of PI3K-PKB-mTOR pathway. J. Hepatol. 2014, 61, 746754, doi:10.1016/j.jhep.2014.05.026.

412. Liu, W.J.; Ye, L.; Huang, W.F.; Guo, L.J.; Xu, Z.G.; Wu, H.L.; Yang, C.; Liu, H.F. p62 links the autophagy pathway and the ubiqutin-proteasome system upon 
ubiquitinated protein degradation. Cell. Mol. Biol. Lett. 2016, 21, 29, doi:10.1186/s11658-016-0031-z.

413.Zhao, Y.; Tao, M.; Wang, R.; Guo, Y.; Wang, M. Japonicone V, a sesquiterpene lactone derivative from the flowers of Inula japonica, inhibits hepatitis $E$ virus replication by targeting virus-associated autophagy. Journal of Functional Foods 2020, 65, 103755, doi:10.1016/j.jff.2019.103755.

414. Seglen, P.O.; Grinde, B.; Solheim, A.E. Inhibition of the lysosomal pathway of protein degradation in isolated rat hepatocytes by ammonia, methylamine, chloroquine and leupeptin. Eur. J. Biochem. 1979, 95, 215-225, doi:10.1111/j.14321033.1979.tb12956.x.

415. Kutsch, M.; Sistemich, L.; Lesser, C.F.; Goldberg, M.B.; Herrmann, C.; Coers, J. Direct binding of polymeric GBP1 to LPS disrupts bacterial cell envelope functions. EMBO J. 2020, 39, e104926, doi:10.15252/embj.2020104926.

416. Barz, B.; Loschwitz, J.; Strodel, B. Large-scale, dynamin-like motions of the human guanylate binding protein 1 revealed by multi-resolution simulations, 2019.

417. Tietzel, I.; El-Haibi, C.; Carabeo, R.A. Human guanylate binding proteins potentiate the anti-chlamydia effects of interferon-gamma. PLoS One 2009, 4, e6499, doi:10.1371/journal.pone.0006499.

418. Fisch, D.; Clough, B.; Domart, M.-C.; Encheva, V.; Bando, H.; Snijders, A.P.; Collinson, L.M.; Yamamoto, M.; Shenoy, A.R.; Frickel, E.-M. Human GBP1 differentially targets Salmonella and Toxoplasma to license recognition of microbial ligands and caspase-mediated death, 2019.

419. Fisch, D.; Bando, H.; Clough, B.; Hornung, V.; Yamamoto, M.; Shenoy, A.R.; Frickel, E.-M. Human GBP1 is a microbe-specific gatekeeper of macrophage apoptosis and pyroptosis. EMBO J. 2019, 38, e100926, doi:10.15252/embj.2018100926.

420. York, A.G.; Williams, K.J.; Argus, J.P.; Zhou, Q.D.; Brar, G.; Vergnes, L.; Gray, E.E.; Zhen, A.; Wu, N.C.; Yamada, D.H.; et al. Limiting Cholesterol Biosynthetic Flux Spontaneously Engages Type I IFN Signaling. Cell 2015, 163, 1716-1729, doi:10.1016/j.cell.2015.11.045.

421.Zhou, Q.D.; Chi, X.; Lee, M.S.; Hsieh, W.Y.; Mkrtchyan, J.J.; Feng, A.-C.; He, C.; York, A.G.; Bui, V.L.; Kronenberger, E.B.; et al. Interferon-mediated reprogramming of membrane cholesterol to evade bacterial toxins. Nat. Immunol. 2020, 21, 746-755, doi:10.1038/s41590-020-0695-4.

422. Kühnl, A.; Musiol, A.; Heitzig, N.; Johnson, D.E.; Ehrhardt, C.; Grewal, T.; Gerke, V.; Ludwig, S.; Rescher, U. Late Endosomal/Lysosomal Cholesterol Accumulation Is a Host Cell-Protective Mechanism Inhibiting Endosomal Escape of Influenza A Virus. mBio 2018, 9, doi:10.1128/mBio.01345-18. 
423. Wang, Q.; Ma, X.; Chen, Y.; Zhang, L.; Jiang, M.; Li, X.; Xiang, R.; Miao, R.; Hajjar, D.P.; Duan, Y.; et al. Identification of interferon- $\gamma$ as a new molecular target of liver X receptor. Biochem. J. 2014, 459, 345-354, doi:10.1042/BJ20131442.

424. Pascual-García, M.; Rué, L.; León, T.; Julve, J.; Carbó, J.M.; Matalonga, J.; Auer, H.; Celada, A.; Escolà-Gil, J.C.; Steffensen, K.R.; et al. Reciprocal negative crosstalk between liver $X$ receptors (LXRs) and STAT1: effects on IFN- $\gamma$-induced inflammatory responses and LXR-dependent gene expression. J. Immunol. 2013, 190, 6520-6532, doi:10.4049/jimmunol.1201393.

425.Zhang, M.A.; Rego, D.; Moshkova, M.; Kebir, H.; Chruscinski, A.; Nguyen, H.; Akkermann, R.; Stanczyk, F.Z.; Prat, A.; Steinman, L.; et al. Peroxisome proliferator-activated receptor (PPAR) $\alpha$ and $-\gamma$ regulate IFN $\gamma$ and IL-17A production by human T cells in a sex-specific way. Proc. Natl. Acad. Sci. U. S. A. 2012, 109, 9505-9510, doi:10.1073/pnas.1118458109.

426. Renga, B.; Migliorati, M.; Mencarelli, A.; Fiorucci, S. Reciprocal regulation of the bile acid-activated receptor FXR and the interferon-gamma-STAT-1 pathway in macrophages. Biochim. Biophys. Acta 2009, 1792, 564-573, doi:10.1016/j.bbadis.2009.04.004.

427. Ma, F.; Liu, S.-Y.; Razani, B.; Arora, N.; Li, B.; Kagechika, H.; Tontonoz, P.; Núñez, V.; Ricote, M.; Cheng, G. Retinoid X receptor $\alpha$ attenuates host antiviral response by suppressing type I interferon. Nat. Commun. 2014, 5, 5494, doi:10.1038/ncomms6494.

428. Cippitelli, M.; Santoni, A. Vitamin D3: a transcriptional modulator of the interferon-gamma gene. Eur. J. Immunol. 1998, 28, 3017-3030, doi:10.1002/(SICI)1521-4141(199810)28:10<3017:AID-IMMU3017>3.0.CO;2-6.

429. Robertson, K.A.; Ghazal, P. Interferon Control of the Sterol Metabolic Network: Bidirectional Molecular Circuitry-Mediating Host Protection. Front. Immunol. 2016, 7, 634, doi:10.3389/fimmu.2016.00634.

430. Andrade, R.J.; García-Escaño, M.D.; Valdivielso, P.; Alcántara, R.; SánchezChaparro, M.A.; González-Santos, P. Effects of interferon-beta on plasma lipid and lipoprotein composition and post-heparin lipase activities in patients with chronic hepatitis C. Aliment. Pharmacol. Ther. 2000, 14, 929-935, doi:10.1046/j.1365-2036.2000.00792.x.

431. Hao, J.; Zhang, Y.-j.; Lv, X.; Xu, N.; Liu, Q.-j.; Zhao, S.; Feng, X.-j.; Xing, L.-1.; Kang, P.-p.; Li, G.-y.; et al. IFN- $\gamma$ induces lipogenesis in mouse mesangial cells via the JAK2/STAT1 pathway. Am. J. Physiol. Cell Physiol. 2013, 304, C760-7, doi:10.1152/ajpcell.00352.2012.

432. Waltl, S.; Patankar, J.V.; Fauler, G.; Nusshold, C.; Ullen, A.; Eibinger, G.; Wintersperger, A.; Kratky, D.; Malle, E.; Sattler, W. 25-Hydroxycholesterol regulates cholesterol homeostasis in the murine CATH.a neuronal cell line. Neurosci. Lett. 2013, 539, 16-21, doi:10.1016/j.neulet.2013.01.014. 
433. Reboul, E.; Dyka, F.M.; Quazi, F.; Molday, R.S. Cholesterol transport via ABCA1: new insights from solid-phase binding assay. Biochimie 2013, 95, 957-961, doi:10.1016/j.biochi.2012.11.009.

434. van den Boomen, D.J.H.; Sienkiewicz, A.; Berlin, I.; Jongsma, M.L.M.; van Elsland, D.M.; Luzio, J.P.; Neefjes, J.J.C.; Lehner, P.J. A trimeric Rab7 GEF controls NPC1-dependent lysosomal cholesterol export. Nat. Commun. 2020, 11, 5559, doi:10.1038/s41467-020-19032-0.

435. Subramanian, K.; Balch, W.E. NPC1/NPC2 function as a tag team duo to mobilize cholesterol. Proc. Natl. Acad. Sci. U. S. A. 2008, 105, 15223-15224, doi:10.1073/pnas.0808256105.

436. Seidah, N.G.; Sadr, M.S.; Chrétien, M.; Mbikay, M. The multifaceted proprotein convertases: their unique, redundant, complementary, and opposite functions. J. Biol. Chem. 2013, 288, 21473-21481, doi:10.1074/jbc.R113.481549.

437. Fabris, C.; Federico, E.; Soardo, G.; Falleti, E.; Pirisi, M. Blood lipids of patients with chronic hepatitis: differences related to viral etiology. Clinica Chimica Acta 1997, 261, 159-165, doi:10.1016/S0009-8981(97)06532-7.

438. Lo, J. Dyslipidemia and lipid management in HIV-infected patients. Curr. Opin. Endocrinol. Diabetes Obes. 2011, 18, 144-147, doi:10.1097/MED.0b013e328344556e.

439. Quaye, O.; Amuzu, B.G.; Adadey, S.M.; Tagoe, E.A. Effect of Hepatitis B Virus (HBV) Infection on Lipid Profile in Ghanaian Patients. Virology (Auckl) 2019, 10, 1178122X19827606, doi:10.1177/1178122X19827606.

440. Daniels, L.B.; Sitapati, A.M.; Zhang, J.; Zou, J.; Bui, Q.M.; Ren, J.; Longhurst, C.A.; Criqui, M.H.; Messer, K. Relation of Statin Use Prior to Admission to Severity and Recovery Among COVID-19 Inpatients. Am. J. Cardiol. 2020, 136, 149-155, doi:10.1016/j.amjcard.2020.09.012.

441. Giguère, J.-F.; Tremblay, M.J. Statin compounds reduce human immunodeficiency virus type 1 replication by preventing the interaction between virion-associated host intercellular adhesion molecule 1 and its natural cell surface ligand LFA-1. J. Virol. 2004, 78, 12062-12065, doi:10.1128/JVI.78.21.1206212065.2004.

442. Mehrbod, P.; Omar, A.R.; Hair-Bejo, M.; Haghani, A.; Ideris, A. Mechanisms of action and efficacy of statins against influenza. Biomed Res. Int. 2014, 2014, 872370, doi:10.1155/2014/872370.

443. Shrivastava-Ranjan, P.; Flint, M.; Bergeron, É.; McElroy, A.K.; Chatterjee, P.; Albariño, C.G.; Nichol, S.T.; Spiropoulou, C.F. Statins Suppress Ebola Virus Infectivity by Interfering with Glycoprotein Processing. mBio 2018, 9, doi:10.1128/mBio.00660-18.

444. Doms, A.; Sanabria, T.; Hansen, J.N.; Altan-Bonnet, N.; Holm, G.H. 25-

Hydroxycholesterol Production by the Cholesterol-25-Hydroxylase Interferon- 
Stimulated Gene Restricts Mammalian Reovirus Infection. J. Virol. 2018, 92, doi:10.1128/JVI.01047-18.

445. Dong, H.; Zhou, L.; Ge, X.; Guo, X.; Han, J.; Yang, H. Antiviral effect of 25hydroxycholesterol against porcine reproductive and respiratory syndrome virus in vitro. Antivir. Ther. 2018, 23, 395-404, doi:10.3851/IMP3232.

446. Liu, S.-Y.; Aliyari, R.; Chikere, K.; Li, G.; Marsden, M.D.; Smith, J.K.; Pernet, O.; Guo, H.; Nusbaum, R.; Zack, J.A.; et al. Interferon-inducible cholesterol-25hydroxylase broadly inhibits viral entry by production of 25-hydroxycholesterol. Immunity 2013, 38, 92-105, doi:10.1016/j.immuni.2012.11.005.

447. Tricarico, P.M.; Caracciolo, I.; Gratton, R.; D'Agaro, P.; Crovella, S. 25hydroxycholesterol reduces inflammation, viral load and cell death in ZIKVinfected U-87 MG glial cell line. Inflammopharmacology 2019, 27, 621-625, doi:10.1007/s10787-018-0517-6.

448. van der Kant, R.; Fish, A.; Janssen, L.; Janssen, H.; Krom, S.; Ho, N.; Brummelkamp, T.; Carette, J.; Rocha, N.; Neefjes, J. Late endosomal transport and tethering are coupled processes controlled by RILP and the cholesterol sensor ORP1L. J. Cell Sci. 2013, 126, 3462-3474, doi:10.1242/jcs.129270.

449. Cinti, A.; Le Sage, V.; Milev, M.P.; Valiente-Echeverría, F.; Crossie, C.; Miron, M.J.; Panté, N.; Olivier, M.; Mouland, A.J. HIV-1 enhances mTORC1 activity and repositions lysosomes to the periphery by co-opting Rag GTPases. Sci. Rep. 2017, 7, 5515, doi:10.1038/s41598-017-05410-0.

450. Balfour, J.A.; McTavish, D.; Heel, R.C. Fenofibrate. A review of its pharmacodynamic and pharmacokinetic properties and therapeutic use in dyslipidaemia. Drugs 1990, 40, 260-290, doi:10.2165/00003495-199040020-00007.

451. Todd, P.A.; Ward, A. Gemfibrozil. A review of its pharmacodynamic and pharmacokinetic properties, and therapeutic use in dyslipidaemia. Drugs 1988, 36, 314-339, doi:10.2165/00003495-198836030-00004.

452.Zhao, Y.D.; Yun, H.Z.H.; Peng, J.; Yin, L.; Chu, L.; Wu, L.; Michalek, R.; Liu, M.; Keshavjee, S.; Waddell, T.; et al. De novo synthesize of bile acids in pulmonary arterial hypertension lung. Metabolomics 2014, 10, 1169-1175, doi:10.1007/s11306014-0653-y.

453. Chang, T.-Y.; Li, B.-L.; Chang, C.C.Y.; Urano, Y. Acyl-coenzyme A:cholesterol acyltransferases. Am. J. Physiol. Endocrinol. Metab. 2009, 297, E1-9, doi:10.1152/ajpendo.90926.2008.

454. Martin, S.; Parton, R.G. Lipid droplets: a unified view of a dynamic organelle. Nat. Rev. Mol. Cell Biol. 2006, 7, 373-378, doi:10.1038/nrm1912.

455. Ehrlich, A.; Uhl, S.; Ioannidis, K.; Hofree, M.; tenOever, B.R.; Nahmias, Y. The SARS-CoV-2 Transcriptional Metabolic Signature in Lung Epithelium. SSRN Journal 2020, doi:10.2139/ssrn.3650499. 
456. Krisans, S.K. The role of peroxisomes in cholesterol metabolism. Am. J. Respir. Cell Mol. Biol. 1992, 7, 358-364, doi:10.1165/ajrcmb/7.4.358.

457. Ribas, V.; García-Ruiz, C.; Fernández-Checa, J.C. Mitochondria, cholesterol and cancer cell metabolism. Clin. Transl. Med. 2016, 5, 22, doi:10.1186/s40169-0160106-5.

458. Medvedev, R.; Ploen, D.; Spengler, C.; Elgner, F.; Ren, H.; Bunten, S.; Hildt, E. $\mathrm{HCV}$-induced oxidative stress by inhibition of Nrf2 triggers autophagy and favors release of viral particles. Free Radic. Biol. Med. 2017, 110, 300-315, doi:10.1016/j.freeradbiomed.2017.06.021.

459. Wang, M.; Casey, P.J. Protein prenylation: unique fats make their mark on biology. Nat. Rev. Mol. Cell Biol. 2016, 17, 110-122, doi:10.1038/nrm.2015.11.

460. Wandinger-Ness, A.; Zerial, M. Rab proteins and the compartmentalization of the endosomal system. Cold Spring Harb. Perspect. Biol. 2014, 6, a022616, doi:10.1101/cshperspect.a022616.

461.Zeyen, L.; Prange, R. Host Cell Rab GTPases in Hepatitis B Virus Infection. Front. Cell Dev. Biol. 2018, 6, 154, doi:10.3389/fcell.2018.00154.

462. Spearman, P. Viral interactions with host cell Rab GTPases. Small GTPases 2018, 9, 192-201, doi:10.1080/21541248.2017.1346552.

463. Elgner, F.; Hildt, E.; Bender, D. Relevance of Rab Proteins for the Life Cycle of Hepatitis C Virus. Front. Cell Dev. Biol. 2018, 6, 166, doi:10.3389/fcell.2018.00166.

464. Caillet, M.; Delcroix, D.; Janvier, K.; Emiliani, S.; Berlioz-Torrent, C. Role of Rab proteins in the formation of HIV-1 particles. Retrovirology 2009, 6, P19, doi:10.1186/1742-4690-6-S2-P19.

465. Goo, Y.-H.; Son, S.-H.; Yechoor, V.K.; Paul, A. Transcriptional Profiling of Foam Cells Reveals Induction of Guanylate-Binding Proteins Following Western Diet Acceleration of Atherosclerosis in the Absence of Global Changes in Inflammation. J. Am. Heart Assoc. 2016, 5, e002663, doi:10.1161/JAHA.115.002663.

466. Smith, A.J.; Mayer, U.; Schinkel, A.H.; Borst, P. Availability of PSC833, a substrate and inhibitor of P-glycoproteins, in various concentrations of serum. J. Natl. Cancer Inst. 1998, 90, 1161-1166, doi:10.1093/jnci/90.15.1161.

467.Shen, F.; Bailey, B.J.; Chu, S.; Bence, A.K.; Xue, X.; Erickson, P.; Safa, A.R.; Beck, W.T.; Erickson, L.C. Dynamic Assessment of Mitoxantrone Resistance and Modulation of Multidrug Resistance by Valspodar (PSC833) in Multidrug Resistance Human Cancer Cells. J. Pharmacol. Exp. Ther. 2009, 330, 423-429, doi:10.1124/jpet.109.153551.

468. Corpechot, C.; Chazouillères, O.; Rousseau, A.; Le Gruyer, A.; Habersetzer, F.; Mathurin, P.; Goria, O.; Potier, P.; Minello, A.; Silvain, C.; et al. A PlaceboControlled Trial of Bezafibrate in Primary Biliary Cholangitis. N. Engl. J. Med. 2018, 378, 2171-2181, doi:10.1056/NEJMoa1714519. 
469. Glitscher, M.; Himmelsbach, K.; Woytinek, K.; Schollmeier, A.; Johne, R.;

Praefcke, G.J.K.; Hildt, E. Identification of the interferon-inducible GTPase GBP1 as major restriction factor for the Hepatitis E virus. J. Virol. 2021, doi:10.1128/JVI.01564-20.

470. Glitscher, M.; Martín, D.H.; Woytinek, K.; Schmidt, B.; Tabari, D.; Scholl, C.; Stingl, J.C.; Seelow, E.; Choi, M.; Hildt, E. Targeting cholesterol metabolism as efficient antiviral strategy against the Hepatitis E virus. Cellular and Molecular Gastroenterology and Hepatology 2021, doi:10.1016/j.jcmgh.2021.02.002. 


\section{Declaration of collaboration}

Except where stated otherwise by reference or acknowledgment, the work presented was generated by myself under the supervision of my advisors during my doctoral studies. All contributions from colleagues are explicitly referenced in the thesis. The material listed below was obtained in the context of collaborative research:

Fig. 33A: HEV-infection triggers changes in cholesterol-related gene-expression and a reduction in intracellular cholesterol.

Collaboration partner: $\quad$ Denna Tabari, Catharina Scholl and Julia C. Stingl Institution:

Federal Institute of Drugs and Medical Devices, Bonn, Germany (Division of Pharmacogenomics)

Contributions: $\quad$ Measurement of differentially expressed genes

My contributions: Sample preparation, formal analysis and data visualisation

Fig. 34: Early HEV-infection triggers reduction of serum-lipids in renal transplant patients.

Fig. 38 A: High intracellular cholesterol reduces viral release, whereas low intracellular cholesterol is in favour of it.
Collaboration partner:
Evelyn Seelow and Mira Choi
Institution:
Charité Universitätsmedizin, Berlin, Germany (Dpt. of Nephrology and Medical Intensive Care)
Contributions:
Patient care and collection of data
My contributions:
Formal analysis and data visualisation

Whenever a figure, table or text is identical to a previous publication, it is stated explicitly in the thesis that copyright permission and/or co-author agreement has been obtained. The following parts of the thesis have been previously published:

All previously published figures were modified from either [469] or [470] and therefore do not violate copyright guidelines.

Frankfurt am Main, 23.0....................

(Date)

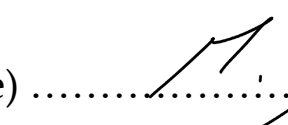

.................(Signature) 


\section{Acknowledgments}

As final remark, I herewith want to thank all of those people who made my past three years and a bit an unforgettable challenge and pleasure and all those who supported me in the efforts of finishing this dissertation.

First and foremost, Prof. Dr. Eberhard Hildt deserves my eternal gratitude. Quite formally, I want to thank you first for giving me the opportunity to work on these projects. I cannot stress enough how big your contribution to me finishing this study was. Not only do I treasure and marvel at your expertise in science, history and random facts, but I also want to express my thankfulness towards you letting me chase every oh so little idea when it comes to experimental planning. Thank you further for long-lasting dialogues - where there could be more than just two people talking - for endless discussions about the for and against of Bafilomycin and for again and again emerging feuds about CMCs. Lastly, I want to thank you for always seeing the good in people, for being the kind-hearted person you are and for your sheer endless patience. Without you I would not be the kind of scientist I believe I am today!

I also want to thank Prof. Dr. Robert Tampé for being my supervisor at university. Surely, I cannot take this for granted and I highly appreciate your efforts in fulfilling your job and taking your time for it. Thank you for pushing me - possibly undeliberately, yet my time as an undergraduate definitely proved to me how highly educated and demanding you are, in a good way - making this dissertation the best that I can.

Further, I thank our designated lab dragon Gert Carra for always being there for me, no matter the time, the stress or the situation he is in. Thank you for always listening, for giving every last bit to keep our lab running and for always growing beyond yourself. Also, huge thanks go to Robin Hobe Murra for running the lab as he does and for sharing the love for bagged dishes.

Thank you, Jasminka Geise, mastress of coins, for not losing your patience with the chaotic herd of people two stories above you. Without your commitment, there would be everything but the progress we are capable of making in work right now. I also want to thank you for cheering me up when things get devastating and for always being ready for a nice little chat, which definitely helps to maintain my sanity.

I want to thank the entire research division 2/01 for being so inclusive, for being such lovely people and for supporting each other. A workplace like this is hard to find and each and every one of you should be proud of being part of it. Thank you, Vanessa Haberger, Catarina Tetherina Sabino, Ines Schmienes Mhedhbi and Michael Basic for being such wonderfully enjoyable office-mates and for every single moment in which we could so efficiently distract one another from taking care of our work. My greatest 
thanks also go to Dr. Catrina Kampuma Spengler, Dr. Tobias Toduac Zahn, an elaborate drama author, Marie-Luise ol' Schmies Herrlein and Dr. Daniela Bender. Thank you for your topic-related discussions, your input and for sharing your knowledge - officially. Unofficially however, I thank you from the bottom of my heart for being such weird people, for being as misanthropic as I am - even you, ol' Schmies -, for having such an infantile humour and for always being up for realising the most awkward ideas there could emerge.

Thanks also goes to all of the students I have supervised in the past years. Thank you for always being so hardly working, for helping me training my own knowledge and for withstanding moods of mine, which surfaced every now and then. Especially, I want to thank Kathrin Woytinek and David Heiler Martín for being astonishingly well-educated, for always keeping up their good work and for the whole lots of fun we had during work. I am grateful to have had people like you who I always could rely on.

I further thank all the scientists who helped me bringing my manuscripts to the point of publication. Thank you for your support and your scientific knowledge and for being so enjoyable to work with.

I want to thank my friends and family for supporting me in every way they could to achieve the goal I have been chasing for the past years. Thank you for your distractions, your love and for being a part of my life. I would not have made it through my studies if it were not for you. Thank you for carrying me through the darkest times and pushing me towards never giving up, as you want nothing but the best for me.

Finally, I want to thank my partner Jean-Philippe Kanter. Thank you for being so patient, so relentlessly supportive, for calming me down and for withstanding every hurdle we are confronted with. Thank you for being my anchor in wuthering surfs and for sharing your life with me. 


\section{Declaration of an oath}

D E C L A R A T I O N

I herewith declare that I have not previously participated in any doctoral examination procedure in a mathematics or natural science discipline.

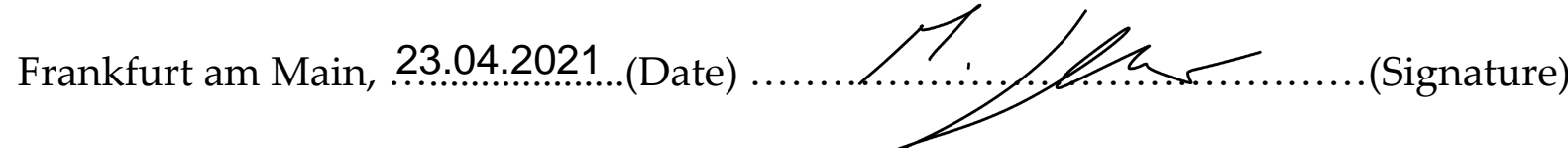

Author's Declaration

I herewith declare that I have produced my doctoral dissertation on the topic of

Endolysosomal effectors and their relevance for antiviral activity against the Hepatitis E virus

independently and using only the tools indicated therein. In particular, all references borrowed from external sources are clearly acknowledged and identified.

I confirm that I have respected the principles of good scientific practice and have not made use of the services of any commercial agency in respect of my doctorate.

Frankfurt am Main, 23.:04.2021...(Date)

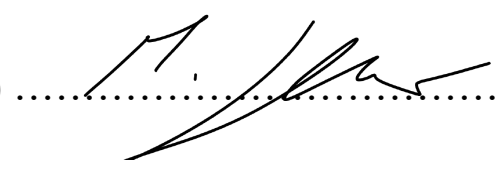

(Signature) 


\section{Publications}

$\underline{\text { Peer-reviewed first author publications }}$

Glitscher M, Martín DH, Woytinek K, Schmidt B, Tabari D, Scholl C, Stingl JC, Seelow E, Choi M, Hildt E. Targeting cholesterol metabolism as efficient antiviral strategy against the Hepatitis E virus. Cell Mol Gastroenterol Hepatol. 2021 Feb 15; S2352345X(21)00035-7. doi: 10.1016/j.jcmgh.2021.02.002.

Glitscher M, Himmelsbach K, Woytinek K, Schollmeier A, Johne R, Praefcke GJK, Hildt E. Identification of the interferon-inducible GTPase GBP1 as major restriction factor for the Hepatitis E virus. J Virol. 2021 Jan 20; JVI.01564-20. doi: 10.1128/JVI.0156420

Glitscher M, Himmelsbach K, Woytinek K, Johne R, Reuter A, Spiric J, Schwaben L, Grünweller A, Hildt E. Inhibition of Hepatitis E Virus Spread by the Natural Compound Silvestrol. Viruses. 2018 Jun 2; 10(6):301. doi: 10.3390/v10060301

\section{$\underline{\text { Peer-reviewed co-author publications }}$}

Kaiser LM, Harms M, Sauter D, Rawat VPS, Glitscher M, Hildt E, Tews D, Hunter Z, Münch J, Buske C. Targeting of CXCR4 by the Naturally Occurring CXCR4 Antagonist EPI-X4 in Waldenström's Macroglobulinemia. Cancers. 2021; 13(4):826. https://doi.org/10.3390/cancers13040826

Peiffer KH, Spengler C, Basic M, Jiang B, Kuhnhenn L, Obermann W, Zahn T, Glitscher M, Loglio A, Facchetti F, Carra G, Kubesch A, Vermehren J, Knop V, Graf C, Dietz J, Finkelmeier F, Herrmann E, Trebicka J, Grünweller A, Zeuzem S, Sarrazin C, Lampertico P, Hildt E. Quadruple mutation GCAC1809-1812TTCT acts as a biomarker in healthy European HBV carriers. JCI Insight. 2020 Nov 19; 5(22):e135833. doi: 10.1172/jci.insight.135833.

Müller C, Obermann W, Schulte FW, Lange-Grünweller K, Oestereich L, Elgner F, Glitscher M, Hildt E, Singh K, Wendel HG, Hartmann RK, Ziebuhr J, Grünweller A. Comparison of broad-spectrum antiviral activities of the synthetic rocaglate CR-31-B (-) and the eIF4A-inhibitor Silvestrol. Antiviral Res. 2020 Mar; 175:104706. doi: 10.1016/j.antiviral.2020.104706. Epub 2020 Jan 10.

Basic M, Elgner F, Bender D, Sabino C, Herrlein ML, Roth H, Glitscher M, Fath A, Kerl T, Schmalz HG, Hildt E. A synthetic derivative of houttuynoid B prevents cell entry of Zika virus. Antiviral Res. 2019 Dec; 172:104644. doi: 10.1016/j.antiviral.2019.104644. Epub 2019 Nov 4. 


\section{Oral presentations}

2018: Retreat on Biomedical Research of the Paul-Ehrlich-Institute, Ronneburg, Germany

Mirco Glitscher, K. Himmelsbach, R. Johne, A. Grünweller and E. Hildt

Inhibition of Hepatitis E virus spread by the natural compound silvestrol

2019: $2^{\text {nd }}$ LOEWE Center DRUID Retreat, Rauischholzhausen, Germany

Mirco Glitscher; Kai-Henrik Peiffer; Stefan Zeuzem and Eberhard Hildt

The fate of the hepatitis E virus capsid and particles is dependent on cholesterol

2020: Retreat on Biomedical Research of the Paul-Ehrlich-Institute, Ronneburg, Germany

Mirco Glitscher; Benjamin Schmidt and Eberhard Hildt

The fate of the Hepatitis E virus capsid and particle is dependent on cholesterol

2021: $30^{\text {th }}$ Annual Meeting of the Society of Virology, Digital conference

Mirco Glitscher; David Heiler Martín; Kathrin Woytinek; Benjamin Schmidt; Denna Tabari; Catharina Scholl; Julia C Stingl; Evelyn Seelow, Mira Choi; Eberhard Hildt

Targeting cholesterol metabolism as efficient antiviral strategy against the Hepatitis E virus

\section{$\underline{\text { Poster presentations }}$}

2018: $28^{\text {th }}$ Annual Meeting of the Society of Virology, Würzburg, Germany

Mirco Glitscher, Kiyoshi Himmelsbach, Reimar Johne, Arnold Grünweller and Eberhard Hildt

Inhibition of Hepatitis E Virus spread by the natural compound silvestrol

2018: $1^{\text {st }}$ LOEWE Center DRUID Retreat, Rauischholzhausen, Germany

Mirco Glitscher, Kiyoshi Himmelsbach, Kathrin Woytinek, Reimar Johne, Andreas Reuter, Jelena Spiric, Luisa Schwaben, Kai-Henrik Peiffer, Arnold Grünweller and Eberhard Hildt

Inhibition of Hepatitis E Virus spread by the natural compound silvestrol 
2019: Retreat on Biomedical Research of the Paul-Ehrlich-Institute, Ronneburg, Germany

Mirco Glitscher, Frederike Tebbe, Philipp Busse, Kiyoshi Himmelsbach, Denna Tabari, Catharina Scholl, Julia Stingl and Eberhard Hildt

Hepatitis E virus egress relies on induction of reactive oxygen species and removal of free cholesterol from host-cells

2019: $29^{\text {th }}$ Annual Meeting of the Society of Virology, Düsseldorf, Germany

M. Glitscher, K. Himmelsbach, A. Schollmeier, C. Kuge, M. Koenig, N. Nouri, G. Praefcke, E. Hildt

The GTPase GBP1 is an antiviral host-factor against HEV and is dysregulated during the virus' life cycle

Mirco Glitscher, Frederike Tebbe, Philipp Busse, Kiyoshi Himmelsbach, Denna Tabari, Catharina Scholl, Julia Stingl and Eberhard Hildt

Hepatitis E virus egress relies on induction of reactive oxygen species and removal of free cholesterol from host-cells

2020: Retreat on Biomedical Research of the Paul-Ehrlich-Institute, Ronneburg, Germany

M. Glitscher, K. Himmelsbach, A. Schollmeier, C. Kuge, M. Koenig, N. Nouri, G. Praefcke, E. Hildt

The GTPase GBP1 is an antiviral host-factor against HEV and is dysregulated during the virus' life cycle

2020: $3^{\text {srd }}$ LOEWE Center DRUID Spring Symposium, Frankfurt, Germany

M. Glitscher, K. Himmelsbach, A. Schollmeier, Kai-Henrik Peiffer, Stefan Zeuzem, G. Praefcke, E. Hildt

The GTPase GBP1 is an antiviral host-factor against HEV and is dysregulated during the virus' life cycle

2021: $30^{\text {th }}$ Annual Meeting of the Society of Virology, Digital conference

Mirco Glitscher; Kiyoshi Himmelsbach; Kathrin Woytinek; Anja Schollmeier; Reimar Johne; Gerrit J. K. Praefcke; Eberhard Hildt

Identification of the interferon-inducible GTPase GBP1 as major restriction factor for the Hepatitis E virus 


\section{Curriculum vitae}

\section{Personal data}

$\begin{array}{ll}\text { Name: } & \text { Mirco Glitscher } \\ \text { Date of birth: } & 16.10 .1993 \\ \text { Place of birth: } & \text { Darmstadt, Germany }\end{array}$

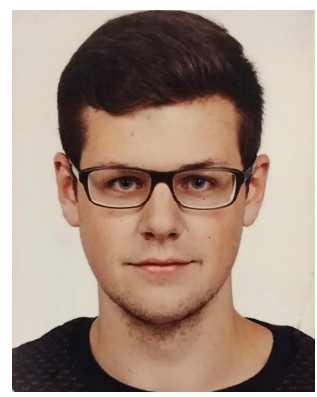

$\underline{\text { Graduate studies }}$

02.2018-01.2021 Doctoral candidate

Paul-Ehrlich-Institute, Langen, Germany

Division 2/01, Virology

Research group: Prof. Dr. Eberhard Hildt

Title: Endolysosomal effectors and their relevance for antiviral activity against the Hepatitis E virus

10.2015 - 01.2018 Master of Science in Biochemistry

Johann-Wolfgang-Goethe University, Frankfurt, Germany

Faculty 14, Biochemistry, Chemistry, Pharmacy

Internship (10.2016-04.2017)

University of Plymouth, Plymouth, United Kingdom

Institute for Translational \& Stratified Medicine

Research group: Ji-Liang Li, MD, PhD

Title: Studying the role of OLFML3 in glioblastoma multiforme.

Thesis (07.2017-01.2018)

Paul-Ehrlich-Institute, Langen, Germany

Division 2/01, Virology

Research group: Prof. Dr. Eberhard Hildt

Title: Characterisation of relevant factors for the life cycle of the Hepatitis E Virus 


\section{Undergraduate studies}

\section{$10.2012-10.2015$ Bachelor of Science in Biochemistry}

Johann-Wolfgang-Goethe University, Frankfurt, Germany

Faculty 14, Biochemistry, Chemistry, Pharmacy

Thesis (04.2015-07.2015)

Paul-Ehrlich-Institute, Langen, Germany

Division 2/01, Virology

Research group: Prof. Dr. Eberhard Hildt

Title: Etablierung eines Zellkultursystems für das Hepatitis E Virus

General higher Education Entrance Qualification

\subsection{4-06.2012 Abitur}

Justus-Liebig-Schule, Darmstadt, Germany 